\title{
Entwicklung optischer Feldmessverfahren zur \\ Charakterisierung mikrofluidischer Mischungsvorgänge
}

\author{
Dissertation zur Erlangung des \\ mathematisch-naturwissenschaftlichen Doktorgrades \\ "Doctor rerum naturalium" \\ der Georg-August-Universität zu Göttingen
}

\author{
vorgelegt von \\ Karsten Roetmann \\ aus Nordhorn
}

Göttingen, 2008 
D7

Referent: Prof. Werner Lauterborn Korreferent: Prof. Gerd Marowsky

Datum der mündlichen Prüfung: 28.03.2008 
meinen Eltern 



\section{Danksagung}

Ich danke Herrn Prof. Dr. Gerd Marowsky für die Möglichkeit meine Promotion am Laser-Laboratorium Göttingen e.V. durchführen zu können und für die Übernahme des Korreferats. Dem Referenten meiner Arbeit, Herrn Prof. Dr. Werner Lauterborn danke ich für die Betreuung von Seiten der Uni. Hoch anzurechnen ist ihm auch die wiederholte Unterstützung bei der Überwindung bürokratischer Hürden.

Bei allen Mitarbeitern des Laser-Labors bedanke ich mich für ein angenehmes Arbeitsklima. Besonderer Dank gebührt dem Leiter der Arbeitsgruppe „Photonische Sensorik“, Dr. Volker Beushausen für die Betreuung meiner Arbeit am Laser-Labor, sowie Alexander Grosch, Konstantin Christou und dem ehemaligen AG-Mitglied Dr. Jochen Scholz für hilfreiche Diskussionen. Auch bei allen weiteren Gruppenmitgliedern bedanke ich mich für die gute Zusammenarbeit und für vergnügliche Aktionen außerhalb der Arbeitszeit.

Die Inhalte dieser Arbeit ergaben sich aus einem Projekt des DFG-Schwerpunktprogrammes 1147, daher danke ich der DFG für die Finanzierung dieses Projektes. Aus dem genannten Schwerpunktprogramm ergaben sich einige wichtige Zusammenarbeiten. Insbesondere gilt mein Dank PD Dr. Christoph Garbe und Daniel Kondermann vom Interdisziplinären Zentrum für Wissenschaftliches Rechnen der Universität Heidelberg für die Anpassung der Algorithmen und die Berechnung vieler vieler Vektorfelder.

Herrn Dr. Günter Rinke vom Institut für Mikroverfahrenstechnik des Forschungszentrums Karlsruhe danke ich für die Bereitstellung des Multilaminationsmischers als Forschungsobjekt.

$\mathrm{Zu}$ guter letzt bedanke ich mich bei meinen Eltern für die Unterstützung, die sie mir gegeben haben und weil sie an mich geglaubt haben. 


\title{
Inhaltsverzeichnis
}

\author{
Abbildungsverzeichnis
}

1. Einleitung 1

2. Mikromischer $\mathbf{5}$

2.1. Modulares Mikrofluidiksystem . . . . . . . . . . . . . 6

2.2. Multilaminationsmischer . . . . . . . . . . . . . . . . . 7

2.3. Flüssigkeitsantrieb . . . . . . . . . . . . . . . . . . . . . . . 9

3. 2D-Molecular Tagging Velocimetry 15

3.1. Die Methode des optischen Flusses . . . . . . . . . . . . . . . . . . 18

3.1.1. Diffusion und Taylordispersion . . . . . . . . . . . . . . . . . 19

3.2. Mustererzeugung . . . . . . . . . . . . . . . . . . . . 22

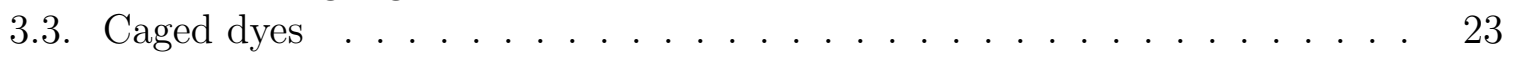

3.3.1. Spektrale Eigenschaften . . . . . . . . . . . . . . . 24

3.3.2. Kinetik des Bindungsbruchs . . . . . . . . . . . . . . 25

3.3.3. Ausbleichen des Farbstoffs . . . . . . . . . . . . . . . . . . . 27

3.3.4. Sättigung der Intensitäten . . . . . . . . . . . . . . . . . 27

3.4. Experimenteller Aufbau . . . . . . . . . . . . . . . . . . . . 30

3.4.1. Masken und Muster . . . . . . . . . . . . . . . . . . . 32

3.5. Quantitative Überprüfung des Verfahrens . . . . . . . . . . . . . . . . 37

3.5.1. 2D-MTV-Messungen . . . . . . . . . . . . . . 38

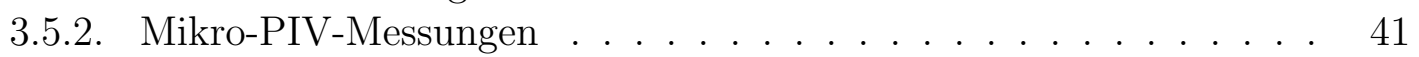

3.6. Messungen in der Mischkammer . . . . . . . . . . . . . . . . . . . . . 44

3.7. Fazit . . . . . . . . . . . . . . . . . . . 46

4. Der Raman-Effekt 49

5. Planare Ramanstreuung $\mathbf{5 5}$

5.1. Prinzip der Messtechnik . . . . . . . . . . . . . . . . 57

5.2. Speziesverteilungen im flachen Mikromischer . . . . . . . . . . . . . . 58

5.3. Steigerung der Intensitäten . . . . . . . . . . . . . . . . . . 59

5.3.1. Anregung .................... 60

5.3.2. Ramanprozess . . . . . . . . . . . . . . . . 63

5.3.3. Optiken und Detektion . . . . . . . . . . . . . . 64

5.4. Verbessertes Detektionssystem . . . . . . . . . . . . . . . 64

5.5. Ramanfilter . . . . . . . . . . . . . . . 66 
5.5.1. Mischung Ethanol-Wasser . . . . . . . . . . . . . . . . . . . 68

5.5.2. Mischung Ethanol-Aceton . . . . . . . . . . . . . . . . . . . 69

5.5.3. Beliebige Mischungen . . . . . . . . . . . . . . . . . 72

5.6. Quantitative Kalibration . . . . . . . . . . . . . . . 73

5.7. Zweidimensionale Messungen . . . . . . . . . . . . . . . . . . . 78

5.7.1. Brechungsindexanpassung . . . . . . . . . . . . 80

5.7.2. Ausgleich von Intensitätsschwankungen und Inhomogenitäten . . 83

5.8. Messungen . . . . . . . . . . . . . . . . . . . . . . . . . . . . . 85

5.8.1. Durchflussmessung . . . . . . . . . . . . . . . . . 87

5.8.2. Dreidimensionale Konzentrationsverteilung im Multilaminationsmischer . . . . . . . . . . . . . . . . . 89

5.9. Fazit . . . . . . . . . . . . . . . . . . . . 99

$\begin{array}{ll}\text { 6. Zusammenfassung und Ausblick } & 101\end{array}$

$\begin{array}{ll}\text { A. Anhang } & 103\end{array}$

A.1. Konstruktionszeichnungen . . . . . . . . . . . . . . . . 103

$\begin{array}{ll}\text { Literaturverzeichnis } & 109\end{array}$ 


\section{Abbildungsverzeichnis}

2.1. Aufbau eines modularen planaren Mikromischers . . . . . . . . . . . . 7

2.2. Verschiedene eingesetzte Geometrien, Mikrokanäle und -mischer . . . . . 8

2.3. Aufbau eines Multilaminationsmischers . . . . . . . . . . . . . . . . 9

2.4. Flüssigkeitsantrieb durch Höhenunterschied . . . . . . . . . . . . . . . . . 10

2.5. Vergleich des Antriebes durch die Spritzenpumpe und durch Höhenunterschied . . . . . . . . . . . . . . . . . . . 11

2.6. Flüssigkeitsantrieb durch Gasdruck . . . . . . . . . . . . . . . . 12

3.1. Prinzip der Geschwindigkeitsmessung mittels MTV . . . . . . . . . . . 16

3.2. Parabelförmiges Strömungsprofil . . . . . . . . . . . . . . . . 20

3.3. Absorption des caged dyes . . . . . . . . . . . . . . . . . . 24

3.4. Intensitätsentwicklung der Fluoreszenz nach Photolyse des caged dyes . . 26

3.5. Intensitätsminderung durch Photobleaching . . . . . . . . . . . 28

3.6. Intensitätsentwicklung bei steigender Leistung des Leselasers . . . . . . . 29

3.7. Intensitätsentwicklung bei steigender Energie des Schreibpulses . . . . . . 30

3.8. Experimenteller Aufbau zur Erfassung von 2D-MTV Sequenzen . . . . . 31

3.9. Taggingmuster kurz nach dem Schreibvorgang . . . . . . . . . . . . . 33

3.10. Mittlere Strömungsgeschwindigkeiten bei schneller Strömung . . . . . . . 34

3.11. Mittlere Strömungsgeschwindigkeiten bei langsamer Strömung . . . . . . 35

3.12. Zeitliche Sequenz des Tagingmusters der schnellen Strömung . . . . . . . 36

3.13. Zeitliche Sequenz des Tagingmusters der langsamen Strömung . . . . . . 36

3.14. Bildsequenz einer MTV-Messung . . . . . . . . . . . . . . . . 37

3.15. Vektorfeld einer MTV-Messung . . . . . . . . . . . . . . . . . 38

3.16. Vektorfeld einer MTV-Messung in einem geraden Mikrokanal . . . . . . . 40

3.17. Vergleich der Ergebnisse der MTV-Messungen mit Referenzwerten . . . . 41

3.18. Experimenteller Aufbau für $\mu \mathrm{PIV-Messungen} \mathrm{.} \mathrm{.} \mathrm{.} \mathrm{.} \mathrm{.} \mathrm{.} \mathrm{.} \mathrm{.} \mathrm{.} \mathrm{.} \mathrm{.} \mathrm{.} \mathrm{.} \mathrm{.} \mathrm{.} 42$

3.19. Partikelbild einer $\mu \mathrm{PIV}$-Messung . . . . . . . . . . . . . . 43

3.20. Vektorfeld einer $\mu$ PIV-Messung in einem gerade Mikrokanal . . . . . . . 43

3.21. Vergleich der Ergebnisse der MTV- und $\mu$ PIV-Messungen mit Referenzwerten . . . . . . . . . . . . . . . . . . 44

3.22. Strömungskonfigurationen in der Mikromischkammer . . . . . . . . . . 45

3.23. Vektorfeld einer MTV-Messung in der Mischkammer eines Mikromischers bei Strömungskonfiguration a . . . . . . . . . . . . . 46

3.24. Vektorfeld einer MTV-Messung in der Mischkammer eines Mikromischers bei Strömungskonfiguration b . . . . . . . . . . . . . 47 
4.1. Energiediagramme zur Ramanstreuung . . . . . . . . . . . . . . . 50

4.2. Einfluss der Polarisationsrichtung des Anregungslichts auf die Intensität des emittierten Ramanstreulichts . . . . . . . . . . . . . . . . 53

5.1. Messung von Speziesdichteverteilungen durch Planare Ramenstreuung . . 57

5.2. Experimenteller Aufbau zur Aufnahme planarer Ramanstreuung . . . . . 59

5.3. Ramanaufnahme der durchströmten Mischkammer, Summierung verschiedener Anzahlen von Laserpulsen . . . . . . . . . . . . . . . . . . . . . . 60

5.4. Steigerung der Intensitätsausbeute durch geänderte Anregung . . . . . . 62

5.5. Optimierter Experimenteller Aufbau zur Aufnahme planarer Ramanstreuung . . . . . . . . . . . . . . . . . . . 65

5.6. Ramanspektren von Wasser und Ethanol und Transmissionsspektrum des Ramanfilters . . . . . . . . . . . . . . . . . . 68

5.7. Ramanspektren von Aceton und Ethanol . . . . . . . . . . . . . . . 69

5.8. Ramanspektren von Aceton und Ethanol mit Transmissionskurve des Filters 70

5.9. Vermessung von Ethanol, Aceton und Wasser mit dem Filter LL-561-25 unter verschiedenen Winkeln . . . . . . . . . . . . . . . . 71

5.10. Vergleich gemessener mit voreingestellten Konzentrationen . . . . . . . . 77

5.11. Zweidimensionale Verteilung der Volumenkonzentration von Ethanol . . . 78

5.12. Intensitätsschwankungen des Lasers . . . . . . . . . . . . . . . . . 79

5.13. Brechungsindexanpassung durch $\mathrm{CaCl}_{2} \ldots \ldots$. . . . . . . . . 80

5.14. Vergleich der Spektren von reinem Wasser und Wasser mit 12,1 \% $\mathrm{CaCl}_{2} \quad 81$

5.15. Messungen mit angepasstem Brechungsindex . . . . . . . . . . . . 82

5.16. Korrektur der Intensitätsschwankungen der Laserstrahlung . . . . . . . . 84

5.17. Strahlhomogenisierung und -formung des Laser Lichtbandes durch Lin-

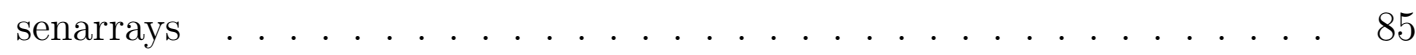

5.18. Messpositionen im Multilaminationsmischer . . . . . . . . . . . 86

5.19. Mittelwertbilder bei einer langsamen Strömungsgeschwindigkeit . . . . . 90

5.20. Bilder der Standardabweichung bei einer langsamen Strömungsgeschwindigkeit . . . . . . . . . . . . . . . . . . . . 91

5.21. Momentaufnahmen der Ethanolverteilung am unteren Ende der Mischstrecke des Mischers bei der langsamen Strömung . . . . . . . . . . . . . 92

5.22. Momentaufnahmen der Ethanolverteilung am unteren Ende der Mischstrecke des Mischers bei der langsamen Strömung . . . . . . . . . . . . . 93

5.23. Mittelwertbilder bei einer schnellen Strömungsgeschwindigkeit . . . . . . 94

5.24. Bilder der Standardabweichung bei einer schnellen Strömungsgeschwin-

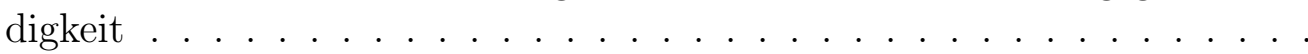

5.25. Momentaufnahmen der Ethanolverteilung am unteren Ende der Mischstrecke des Mischers bei der schnellen Strömung . . . . . . . . . . . .

5.26. Momentaufnahmen der Ethanolverteilung am unteren Ende der Mischstrecke des Mischers bei der schnellen Strömung . . . . . . . . . . . . .

5.27. Momentaufnahmen der Ethanolverteilung am oberen Ende der Mischstrecke des Mischersbei der schnellen Strömung . . . . . . . . . . . . . 
5.28. Momentaufnahmen der Ethanolverteilung oberhalb $\mathrm{x}=15 \mathrm{~mm}$ bei der schnellen Strömung . . . . . . . . . . . . . . . . . . . . . 99

A.1. Konstruktionszeichnung des Rahmens, Bauteil oben . . . . . . . . . . . . 104

A.2. Konstruktionszeichnung des Rahmens, Bauteil unten . . . . . . . . . . 105

A.3. Konstruktionszeichnung der Quarzglasplatten . . . . . . . . . . . . 106

A.4. Konstruktionszeichnung des großen Mikromischers . . . . . . . . . . . . . 107

A.5. Konstruktionszeichnung des kleinen Mikromischers . . . . . . . . . . 108 


\section{Einleitung}

Einige der größten technologischen Durchbrüche der letzten Jahrzehnte verdanken wir dem Prozess der Miniaturisierung. Begriffe wie „Mikroelektronik“, „Mikrooptik“ und „Mikrofluidik" haben Einzug in unseren Sprachgebrauch gefunden. Inzwischen werden sogar erste Schritte im Bereich der "Nanotechnologie“ unternommen. Vor allem miniaturisierte elektronische Systeme sind heute allgegenwärtig. Computer beruhen auf der Funktionsweise von Transistoren. Diese wurden in wenigen Jahrzehnten in nahezu unvorstellbarem Tempo immer weiter verkleinert. Nachdem in den 40er Jahren des letzten Jahrhunderts die ersten funktionierenden Transistoren konstruiert wurden, begann eine rasante Erfolgsgeschichte der Miniaturisierung. Die Größe heutiger Transistoren bewegt sich im zweistelligen Nanometerbereich, so dass auf einem aktuellen Computerprozessor (Intel Itanium 2 Montecito, 2006) fast zwei Milliarden Transistoren integriert werden konnten. Die Größe des gesamten Systems liegt dabei mit wenigen Zentimetern noch unter derjenigen eines einzelnen Transistors zu Beginn seiner Entwicklungsgeschichte. Folge der fortschreitenden Entwicklung sind enorme Kostensenkungen und damit einhergehend eine weite Verbreitung moderner Computersysteme, sowie eine beträchtliche Leistungssteigerung.

Auch im Bereich der Optik gab es bedeutende Erfolge durch Miniaturisierungen. Der Laser hat nach seiner Erfindung im Jahr 1960 schnell Einzug in unseren Alltag gehalten. Winzige Lasersysteme finden sich heute zum Beispiel in CD-Playern, Barcodelesern oder Laserdruckern. Die Miniaturisierung ermöglichte auch in der Medizinischen Optik wichtige Durchbrüche wie das Endoskop, mit dem heute Diagnosen im Körperinneren ohne Operation möglich sind.

Ein weiterer Bereich der Miniaturisierung ist die Mikrofluidik. Die Vorteile mikrofluidischer Systeme sind bestechend. Insbesondere Applikationen im Bereich der chemischen und biochemischen Analytik sowie der klinischen Diagnostik, bei denen oft nur winzigste Probenmengen verfügbar sind, profitieren von den kleinen Kanalabmessungen und deutlich verkürzten Analysezeiten. Die Fiktion der so genannten "Labs-on-a-chip“ beginnt nach und nach Wirklichkeit zu werden. Handgroße Analyselabore könnten in Zukunft in der Lage sein, die Aufgaben aufwändiger Analyseketten zu übernehmen, die derzeit noch mehrere Laborräume füllen. Mikrofluidische Systeme werden zunehmend auch für chemische Produktionsabläufe interessanter. Die Möglichkeit, die physikalischen Randbedingungen der jeweiligen Prozessschritte, aufgrund des großen Oberfläche-zu-VolumenVerhältnisses, definiert einstellen und kontrollieren zu können, ermöglicht eine effizientere Produktion mit weniger Nebenprodukten. Die effiziente Abführung von Reaktionswärme ermöglicht in mikrofluidischen Dimensionen die Durchführung bestimmter exothermer Reaktionen. Sogar Reaktionen, die in makroskopischen Systemen explosiv ablaufend würden, könnten in mikrofluidischen Systemen gebändigt werden. Die Produkti- 
on vollkommen neuer Reagenzien wird damit durch die Mikrofluidik erst möglich. Bei klassischem Vorgehen werden neue chemische Produktionsketten üblicherweise zuerst in kleinerem Maßstab im Labor getestet. Anschließend werden die einzelnen Prozessschritte erheblich vergrößert für die Massenproduktion umgesetzt. Es kommt jedoch immer wieder vor, dass die Unterschiede durch die Größenänderung unterschätzt werden, und die Reaktionen im Großen nicht so ablaufen wie bei den Voruntersuchungen im Labor. Wenn die Produktion dagegen durch mikrofluidische Systeme geschieht, treten diese Probleme nicht auf, da mikrofluidische Mischer und Reaktoren die notwendigen Durchsätze durch Parallelisierung vieler Einzelsysteme erreichen. Jedes dieser Systeme ist weiterhin identisch zu denjenigen, die bei den Voruntersuchungen im Labor genutzt wurden. Die Parallelisierung sorgt gleichzeitig für mehr Sicherheit, da im Zweifelsfall nur einzelne Mikrosysteme außer Kontrolle geraten. Die Miniaturisierung fluidischer Komponenten und ihr Einsatz in den genannten Gebieten kommt gerade erst in Fahrt. Vor allem Fortschritte in der Fertigung mikrofluidischer Komponenten führen aber inzwischen zu einer rasanten technologischen Entwicklung.

Insbesondere die Entwicklung von effizienten Mikromischern hat einen hohen Stellenwert. Da sowohl Diagnose- als auch Produktionsprozesse in den meisten Fällen auf chemischen Reaktionen beruhen, ist eine schnelle und vollständige Vermischung der Reaktanden ausgesprochen wichtig. Gerade Mischsysteme lassen sich jedoch nicht einfach verkleinern, ohne ihre Funktionalität zu verlieren. Dies liegt vor allem daran, dass im Vergleich zu makroskopischen Systemen in mikroskopisch kleinen Dimensionen andere physikalische Gesetzmäßigkeiten dominieren. In makroskopischen Dimensionen spielt die Turbulenz der Fluide eine entscheidende Rolle für eine effiziente Mischung. In Mikroströmungen ist der Bereich der Turbulenz dagegen kaum zu erreichen, die Mischprozesse laufen daher rein diffusiv ab. Um die Aufgabe der Vermischung von Fluiden auch in mikroskopischen Dimensionen zu erfüllen, sind folglich vollkommen neue Konzepte notwendig.

Aufgrund des gestiegenen und ständig weiter wachsenden Interesses an mikrofluidischen Systemen besteht großer Bedarf an neuen diagnostischen Hilfsmitteln zur Analyse der Strömung und des Mischungs- und Reaktionsverhaltens verschiedenster Fluide in Mikrokanälen. Besonders berührungslos arbeitende Messverfahren sind in den winzigen Dimensionen dieser Systeme von besonderem Interesse, da eine Beeinflussung der Strömungen und Reaktionen ausgeschlossen werden muss. Eine grundlegende Erforschung und Optimierung der Vorgänge in Mikromischern ist eine der wichtigsten Aufgaben neuer Analysemethoden.

Im Verlauf der hier beschriebenen Arbeiten wurden daher neue Messverfahren entwickelt, durch die eine umfassendere Analyse von mikrofluidischen Mischungsvorgängen ermöglicht wird. Diese Verfahren sind in der Lage, quantitative, flächige Strömungsgeschwindigkeiten und Spezieskonzentrationen zu bestimmen. Die Kombination eines Geschwindigkeitsmessverfahrens mit einem Verfahren zur Bestimmung von Konzentrationsverteilungen ermöglicht eine sehr umfassende Analyse von Gemischbildungsvorgängen. Sowohl die momentane Verteilung der beteiligten Fluide kann damit bestimmt werden, als auch die Dynamik der zugrunde liegenden Mischprozesse. Für die Bestimmung 
von Geschwindigkeitsfeldern wird hier die klassische „Molecular Tagging Velocimetry“ (MTV) zu einem zweidimensional ortsauflösenden Messverfahren für die Mikrofluidik weiterentwickelt. Das zweite Messverfahren soll in der Lage sein quantitative Konzentrationsverteilungen auf Grundlage der planaren spontanen Ramanstreuung zu erfassen. 


\section{Mikromischer}

Mikromischer spielen eine wichtige Rolle in den verschiedensten Bereichen der Mikrofluidik. In mikrofluidischen Systemen geschehen Mischvorgänge ausschließlich durch die Diffusion der Fluide, da durch die winzigen Systemdimensionen und die dadurch extrem kleinen Reynoldszahlen nur laminare Strömungen generiert werden. Turbulente Mischvorgänge sind in den meisten Fällen nicht realisierbar. Damit eine mikrofluidische Mischung unter diesen Bedingungen effizient und schnell realisiert werden kann, muss der Mischprozess unterstützt werden. Es existiert eine Vielzahl unterschiedlichster Ansätze um die Diffusion effektiver zu nutzen, bzw. zu unterstützen. Im Prinzip findet sich in dieser Formulierung bereits die grundlegendste Unterteilung von Mikromischern in aktive und passive Konzepte. Bei passiven Mikromischern wird während der Mischung nicht in das System eingegriffen. Für eine effektivere Mischung wird die Strömungsgeometrie so modifiziert, dass die Grenzfläche zwischen den Fluiden vergrößert wird oder zusätzliche Scherkräfte aufgeprägt werden [39, 40, 41]. Bei aktiven Mischern wird die, für eine schnelle Vermischung notwendige, Grenzflächenvergrößerung durch den Einsatz einer oder mehrerer externer Erregungen realisiert, die im Wesentlichen eine Faltung der Grenzfläche bewirken. Die Anregung kann dabei nahezu beliebig realisiert werden. Es existieren zum Beispiel druckgetriebene [6], elektrische [14, 52] oder magnetische [68] Anregungen. Eine sehr umfassende Auflistung und detaillierte Einteilung von Mikromischern findet sich in [51]. Mikromischer und weitere Komponenten werden auf unterschiedlichste Art und Weise gefertigt [27, 58]. Einen Überblick über die gängigsten Techniken bieten [4, 47], in [4] sind zusätzlich die physikalischen Grundlagen mikrofluidischer Strömungen und Mischungen erläutert. Neben der vollständigen Vermischung ist bei analytischen Systemen die Handhabung kleinster Probenmengen von Interesse. In diesem Fall ist eine vollständige Mischung nicht unbedingt erwünscht oder notwendig. Für eine Reaktion ist aber trotzdem eine minimale Vermischung notwendig [8, 23]. Allgemeine Überlegungen zu den Möglichkeiten und Grenzen der Miniaturisierung wurden in [45] angestellt. Einen umfangreichen Überblick über mikrofluidische Systeme erhält man in [64] und [15].

Eine effektive Mischung ist aus Sicht der Anwendung die wichtigste Anforderung an einen Mikromischer. Für die Entwicklung optischer Messtechniken ist dies jedoch weniger entscheidend. Für die in dieser Arbeit durchgeführte Entwicklung optischer Messverfahren wird daher ein möglichst einfaches und möglichst gut optisch zugängliches Modell-Strömungssystem eingesetzt. Es ist sinnvoll erst nach und nach die höheren Anforderungen realer Mischsysteme an die Messtechnik zu beachten. Auch ein einfaches Modellsystem sollte dabei noch möglichst anwendungsnah aufgebaut sein, damit die Eigenschaften der Mikromischer bei der Entwicklung berücksichtigt werden können. Gewisse Anforderungen werden auch nach Aufbau und Optimierung des neuen Messverfahrens bestehen bleiben, wie zum Beispiel in diesem Fall die optische Transparenz. Mit einem 
funktionierenden Verfahren ist es aber unter Umständen möglich, die Anforderungen auf ein Minimum zu reduzieren, so dass zum Beispiel nur kleine Bereiche optisch transparent bleiben müssen. Neben dieser grundlegenden Forderung der optischen Zugänglichkeit ist es für die hier angestellten Entwicklungsarbeiten zudem von Vorteil, ein einfaches modulares System zu verwenden. Damit wird z.B. eine Reinigung, vor allem der optischen Zugänge erleichtert und es ist bei entsprechender Planung möglich, die Strömungsgeometrie schnell zu verändern.

Im Verlauf der Entwicklung der optischen Messtechniken kamen insbesondere zwei Systeme zum Einsatz. Das erste wurde vor allem zur Entwicklung der Geschwindigkeitsmessung eingesetzt, es wurden aber auch erste Konzentrationsmessungen daran durchgeführt. Da im Fall der Geschwindigkeitsmesstechnik eine Vermischung mehrerer Fluide nicht unbedingt notwendig ist, wurde das System meist nur mit einer Flüssigkeit genutzt. Das zweite System wurde bei der Entwicklung der Messtechnik zur Bestimmung von Konzentrationsverteilungen genutzt. Die Strömungen der eingesetzten Flüssigkeiten wurden ausschließlich druckgetrieben realisiert.

Neben den druckgetriebenen Flüssigkeitsantrieben stellt der elektrokinetische Antrieb eine wichtige Alternative im Bereich mikrofluidischer Strömungen dar. Vor allem elektrophoretische [13, 63] und elektroosmotische [9, 12, 61, 66, 77] Antriebe sind weit verbreitet. Ein Einsatz dieser Techniken wäre in dieser Arbeit nicht ganz einfach, da an vielen Stellen Metall als Material eingesetzt wurde. Für die Entwicklung der Messtechniken ist die Beschränkung auf druckgetriebene Strömungen unproblematisch, daher wurde auf diesen Bereich verzichtet.

\subsection{Modulares Mikrofluidiksystem}

Dieses mikrofluidische System wurde so konzipiert, dass unterschiedliche Strömungsgeometrien realisiert werden können. Das System muss dabei nicht unbedingt die Funktion eines Mikromischers einnehmen. Der Aufbau ist aus wenigen Teilen zusammengesetzt, so dass eine Änderung des Strömungsverlaufs mit wenig Aufwand möglich ist. Eine Explosionszeichnung der zugehörigen Teile ist in Abbildung $2.1 \mathrm{zu}$ finden. In der Mitte befindet sich eine Folie, die die gewünschte Kanalstruktur enthält. Dabei wird durch die Dicke der Folie die Höhe der Strömungskanäle vorgegeben. Je nach Herstellungsprozess können dafür unterschiedliche Materialien zum Einsatz kommen. Nach oben und unten werden die Kanäle durch Quarzglasplatten abgeschlossen. Das Glas bietet optimale optische Zugänglichkeit in einem weiten Bereich und von zwei Seiten. Der spektrale Transmissionsbereich des Quarzglases ist zudem sehr groß, so dass unter anderem die Verwendung von UVStrahlung ohne nennenswerte Verluste möglich ist. In der oberen Glasplatte befinden sich Bohrungen für die fluidischen Zugänge. Auf diese Bohrungen wurden Anschlüsse geklebt, die direkt mit Schläuchen verbunden werden können. Zusammengehalten und gleichzeitig abgedichtet werden die Kanäle durch einen zweiteiligen Edelstahlrahmen, der mittels Schrauben zusammengepresst werden kann. Damit der Druck gleichmäßig auf die Glasplatten verteilt wird, reicht der Rahmen rund um den Rand der Platten. Der verbleibende Spalt zwischen den Glasplatten und den Mikrokanälen wird durch die Flüssigkeit gefüllt 


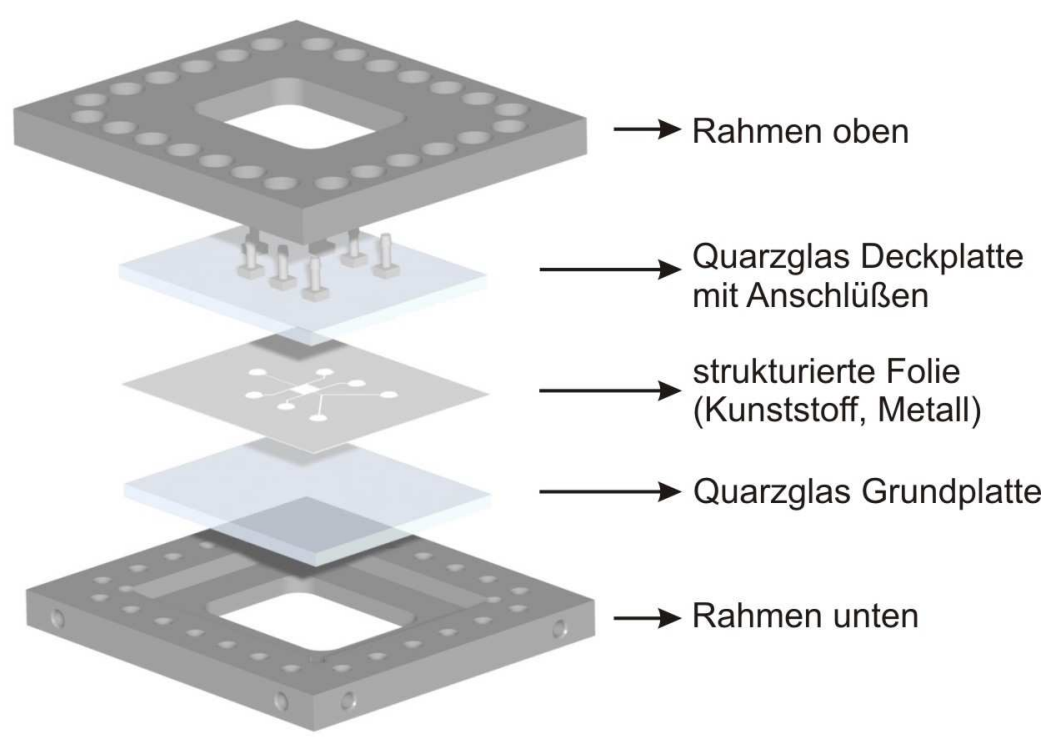

Abb. 2.1.: Aufbau eines modularen planaren Mikromischers

und durch Adhäsion vollständig verschlossen. Da durch diesen Aufbau die Verwendung von zusätzlichen Dichtmitteln nicht notwendig ist, kann das System problemlos jederzeit in seine Einzelteile zerlegt und wieder zusammengesetzt werden.

In Abbildung 2.2 sind verschiedene Geometrien der Kanalfolien gezeigt, wie sie später bei den Messungen eingesetzt wurden. Auf der linken Seite befinden sich gerade Strömungskanäle, die zu einer Überprüfung der gemessenen Strömungsgeschwindigkeiten besonders geeignet sind. Die Breite des oberen Kanals liegt bei $5 \mathrm{~mm}$, die des unteren bei $1 \mathrm{~mm}$. Auf der rechten Seite sind zwei Mikromischer zu finden, die eine Vielzahl unterschiedlicher Nutzungsvarianten ermöglichen. Die Form des Mischers ist angelehnt an einen ähnlichen Mikromischer in [52]. Die Mischkammer ist im oberen Fall $5 \times 5 \mathrm{~mm}$ groß und die Kanäle sind 1,5 mm breit. Unten ist die Kammer $1 \times 1 \mathrm{~mm}$ groß und die Kanäle sind 0,3 mm breit. Jedes Element misst insgesamt $50 \times 60 \mathrm{~mm}$ und passt damit genau in den vertieften Bereich des Rahmens in Abbildung 2.1. Als Trägermaterial wurden Edelstahlfolien der Dicke 0,2 mm verwendet. Für die Herstellung wurden die Strukturen von der Firma RST-Cutdesign GmbH in Braunschweig fotolithografisch von einer digitalen Vorlage übertragen und anschließend geätzt.

\subsection{Multilaminationsmischer}

Der zweite verwendete Mikromischer wurde nicht im Verlauf der Arbeiten entwickelt, sondern lediglich an die Anforderungen der Messtechnik angepasst. Der Mischer selbst wurde zur Verfügung gestellt vom Institut für Mikroverfahrenstechnik des Forschungszentrums Karlsruhe. Der Mikromischer nutzt passiv verstärktes Mischen durch Multi- 

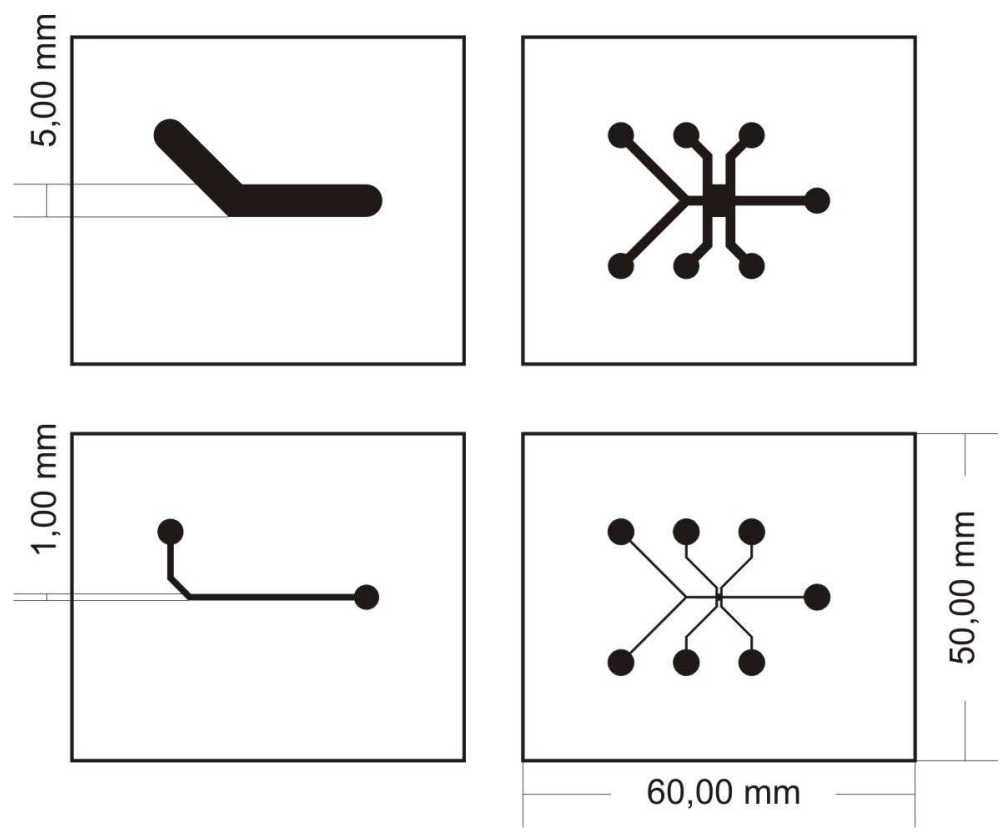

Abb. 2.2.: Verschiedene eingesetzte Geometrien, Mikrokanäle und -mischer

lamination. Zu diesem Zweck werden die zugeleiteten Flüssigkeiten in viele kleine Filamente aufgeteilt, so dass die Kontanktfläche deutlich vergrößert wird. Weiterhin sind die Auslässe der beiden Flüssigkeiten unter einem Winkel von $90^{\circ}$ angeordnet, so dass zusätzliche Scherkräfte aufgeprägt werden. In Abbildung 2.3 ist der Mikromischer und eine genauere Zeichnung der Auslässe zusammengestellt. Die beiden verwendeten Flüssigkeiten erreichen den Mischer durch die Anschlüsse im unteren Bereich. Der Mischer selbst besteht aus mehreren Stahlplättchen, in die mittels Mikrofrästechniken die Kanalstrukturen eingebracht wurden. Diese Plättchen wurden danach durch zwei Schrauben zu einem kompakten Barren zusammen gepresst und damit abgedichtet. Dieser Barren bildet das zentrale Element, welches die Aufteilung der Flüssigkeiten in viele dünne Filamente übernimmt. Von den Zuläufen fließen die Flüssigkeiten durch die eingefrästen Kanäle unter einem $45^{\circ}$-Winkel zu den Auslässen. Die Anordnung dieser vier mal vier Kanäle ist in der linken Hälfte der Abbildung zu finden. Die Zahl der Auslasskanäle ist hier bewusst gering gehalten. Zum einen dient der Mischer der grundlegenden Untersuchung der Mischprozesse dieses Mischertyps und der Überprüfung numerischer Simulationen, für die ein einfaches System von Vorteil ist. Zum anderen stellt ein einfaches System geringere Anforderungen an die neu zu entwickelnde Messtechnik. Es existieren weitere Versionen des Mikromischers mit deutlich mehr Auslasskanälen, die eine effektivere Vermischung ermöglichen sollen. Im Nachlauf der Auslässe beginnt der eigentliche Mischbereich. Die Mischstrecke befindet sich innerhalb eines Quarzglaskanals. Für diesen Kanal wurde eine Standard-Quarzglasküvette ohne Boden verwendet. Diese Küvetten können kostengünstig bezogen werden und besitzen optimale optische Eigenschaften um 


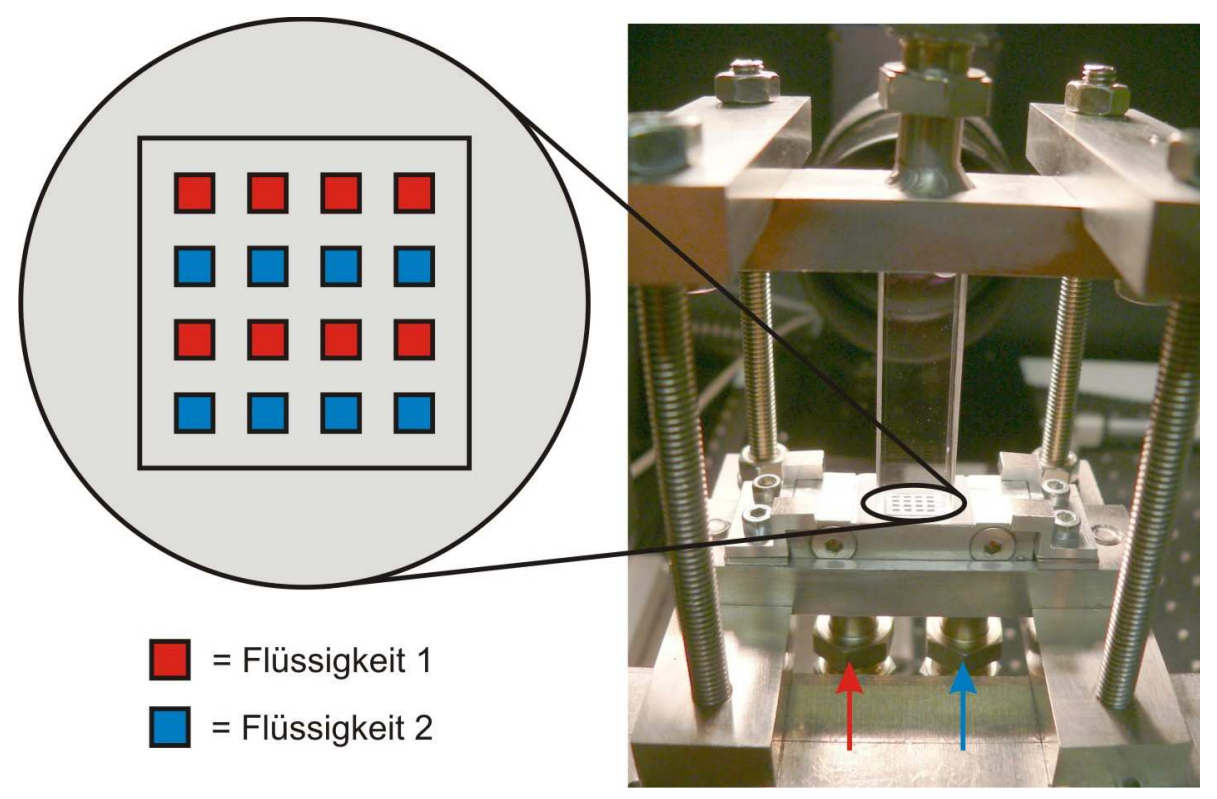

Abb. 2.3.: Aufbau des Prototypen eines Multilaminationsmischers

einen optischen Zugang von allen vier Seiten zu ermöglichen. Am Ende der Mischstrecke fließen die Flüssigkeiten in einen Abfluss. Der Zu- und Abflussanschluss sind über Gewindestangen miteinander verbunden, so dass das System mit leichtem Druck verschraubt und fixiert werden kann. Die Gewindestangen sind so angeordnet, dass sie die optischen Wege in die Küvette nicht verdecken.

Die Auslässe liegen in einem Bereich, der ca. $0,5 \mathrm{~mm}$ höher liegt als die restliche Oberfläche des Barrens. Damit ist es mögliche, die Küvette unterhalb der Oberfläche der Auslässe abzudichten und trotzdem einen optimalen optischen Zugang bis direkt an die Auslässe zu gewährleisten. Die Ränder der Küvette liegen etwas unterhalb der Auslässe, damit Abschattungen der optischen Wege durch die Ränder vermieden werden. Am Ende der Mischstrecke wird die Küvette in eine Vertiefung am Abfluss gesteckt. Abgedichtet wird der Bereich an beiden Enden durch Kunststoffdichtungen.

\subsection{Flüssigkeitsantrieb}

Jeder der verwendeten Mikromischer und -kanäle benötigt einen Flüssigkeitsantrieb. Auch die Anforderungen an diesen Bereich sind je nach Fragestellung und eingesetzter Messtechnik hoch. Vor allem bei der Entwicklung einer Geschwindigkeitsmesstechnik sind gleichmäßige, also pulsationsfreie Strömungen eine wichtige Voraussetzung. Im Verlauf der Entwicklung der Messtechniken wurden auch bei den Flüssigkeitsantrieben wertvolle Erfahrungen gesammelt, so dass die Art des Antriebs optimiert und an die jeweilige Messtechnik angepasst werden konnte. Die verwendeten Mikromischer und -kanäle un- 


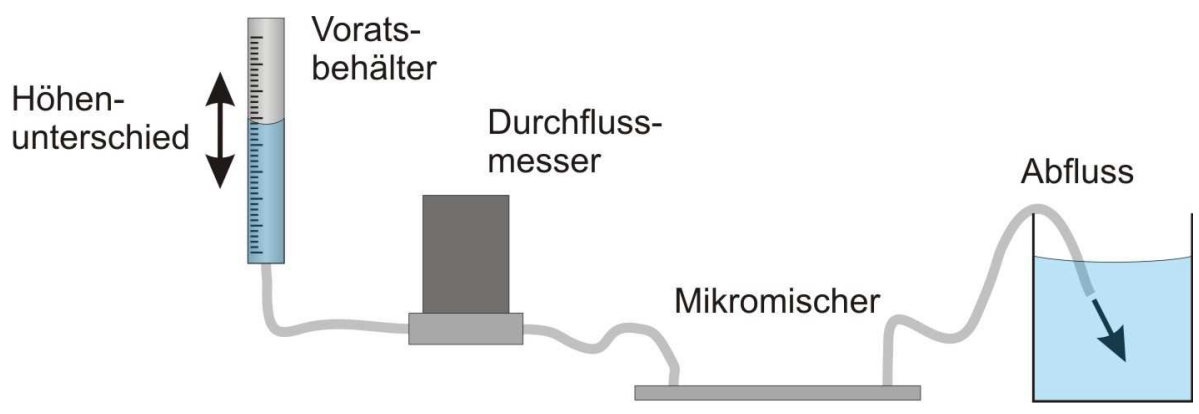

Abb. 2.4.: Flüssigkeitsantrieb durch Höhenunterschied

terscheiden sich dabei so sehr, dass für jede Messtechnik ein unterschiedliches Verfahren eingesetzt wurde. Zunächst wurde eine Spritzenpumpe verwendet, die jedoch nicht in der Lage ist, eine pulsationsfreie Strömung zu garantieren. Dies resultiert einerseits daraus, dass die verwendeten Elektromotoren der Spritzenpumpen nicht vollständig gleichförmig laufen und andererseits können sich in den Spritzen durch die entstehenden Reibungskräfte Spannungen aufbauen, die sich mehr oder weniger periodisch entladen. Es ist daher sehr wichtig, Spritzen zu verwenden, die sich nicht verformen können. Auch starre Glasspritzen können nicht vollständig verhindern, dass zwischen dem Kolben und dem Zylinder der Spritze Spannungen entstehen, da hier ein Dichtmaterial unumgänglich ist. Selbst wenn die Spritzen vollkommen starr aufgebaut wären und der Kolben reibungsfrei liefe, würden noch Pulsationen auftreten, da der Kolben in den meisten Fällen durch einen Schrittmotor angetrieben wird. Bei den extrem geringen Durchflüssen, die bei den untersuchten Strömungen verwendet wurden, erreicht man auch bei Verwendung von Schrittmotoren mit hoher Schrittzahl die Grenzbereiche, in denen ein gleichmäßiger Betrieb kaum mehr möglich ist. Die Spritzenpumpe wurde daher nur für Untersuchungen verwendet, die in dieser Hinsicht unkritisch waren.

Eine weitere Möglichkeit ist ein Flüssigkeitsantrieb durch Gasdruck. Die Reservoirs der Flüssigkeiten werden dazu mit einem Gasanschluss versehen. Ein geringer, gleich bleibender Überdruck sorgt für einen gleichmässigen Fluss. Die Durchflussmenge kann bei dieser Variante auf zwei Arten eingestellt werden. Entweder wird der Gasdruck sehr fein reguliert, oder die Flüssigkeit selbst wird durch Einsatz eines Drosselventils entsprechend gebremst. Die Druckunterschiede, die für eine genaue Einstellung sehr geringer Durchflussmengen notwendig sind, sind ebenfalls winzig. Eine entsprechend präzise Regelung der Gasdrücke lässt sich nur sehr aufwändig realisieren. Die zweite Einstellungsvariante durch Drosselventile im Flüssigkeitsstrom ist erheblich einfacher und dementsprechend kostengünstiger zu realisieren. Die beiden eingesetzten Systeme, die in den vorangegangenen Abschnitten vorgestellt wurden, unterscheiden sich deutlich im Aufbau und wurden zudem bei der Entwicklung der Messtechniken unterschiedlich eingesetzt. Im Fall des modularen Mikrofluidiksystems wurden sehr kleine Durchflüsse von nur wenigen Mikrolitern pro Minute verwendet, während beim Multilaminationsmischer deutlich größere 


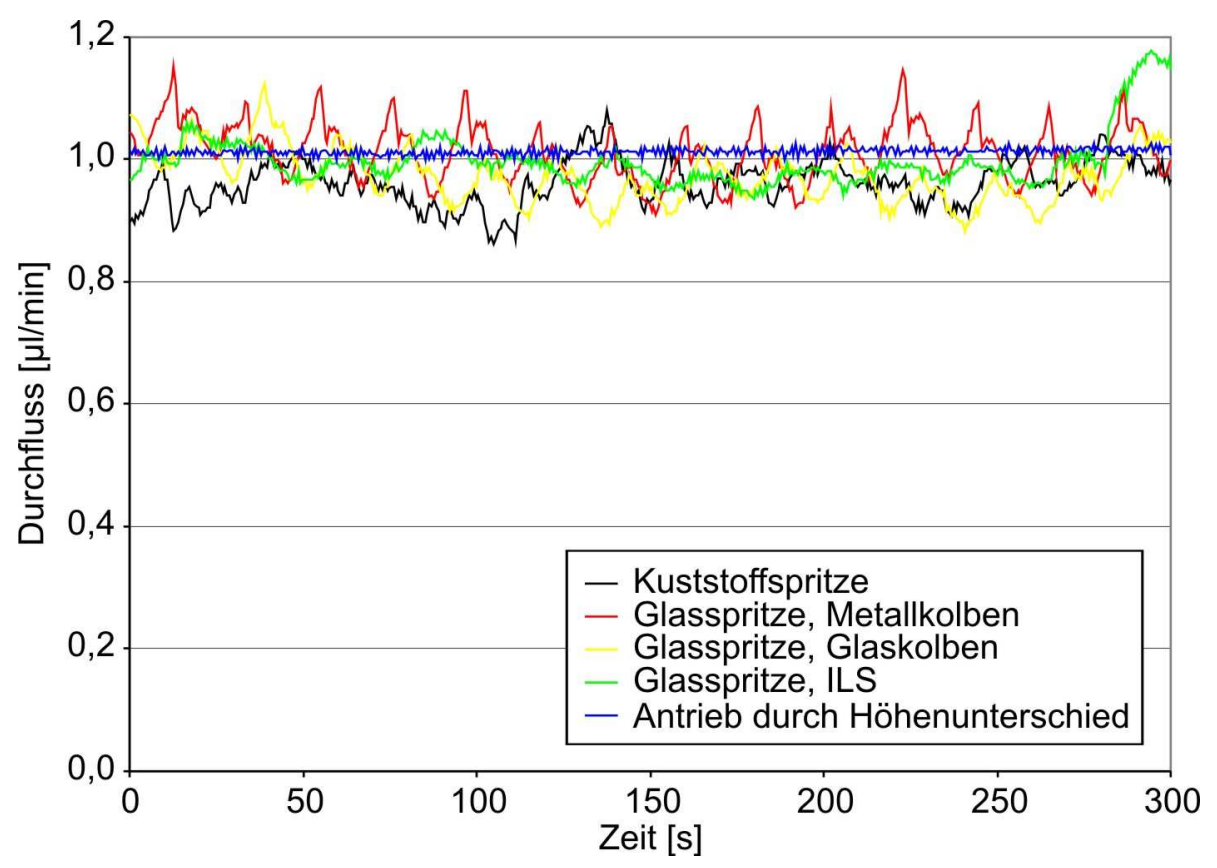

Abb. 2.5.: Vergleich des Antriebes durch die Spritzenpumpe (verschiedene Spritzen) und durch Höhenunterschied

Durchflussmengen im Bereich von 10 bis $100 \mathrm{ml} / \mathrm{min}$ genutzt wurden. Zunächst wurden in beiden Systemen die Durchflüsse durch Ventile im Flüssigkeitsstrom reguliert. Es stellte sich heraus, das die Durchflusswerte im unteren Bereich auch durch hochpräzise Nadelventile nicht fein genug einstellbar waren. Für den Flüssigkeitsantrieb am modularen System wurde daher auf eine andere Technik ausgewichen. Die einfachste Möglichkeit, extrem geringe Druckunterschiede präzise einzustellen, ist, den Höhenunterschied zwischen den Flüssigkeitsspiegeln im Zulauf- und Abflussreservoir zu verändern. Dieses Vorgehen ist in Abbildung 2.4 skizziert. Das Reservoir wird höhenverstellbar angebracht, Ventile oder ähnliches fehlen vollständig. Zur Kontrolle wird der Durchfluss mit einem hochpräzisen Durchflussmesser (SLG1430-320, Sensirion GmbH) gemessen. Im Bereich von wenigen Mikrolitern pro Minute funktionieren die meisten bekannten Messprinzipien nicht mehr. Der verwendete Durchflussmesser ist ein spezielles Gerät, das ein thermisches Messprinzip verwendet. Dabei erzeugt ein nahezu punktförmiges Heizelement einen Temperaturgradienten in der strömenden Flüssigkeit, die sich in einem Rohr mit bekanntem und unveränderlichem Querschnitt befindet. Der Temperaturgradient wird durch je einen Thermosensor vor und hinter dem Heizelement vermessen. Bei einem bestehenden Durchfluss verändert sich der Temperaturgradient und die Sensoren stellen eine Temperaturänderung fest. Durch entsprechende Kalibrierung können die Temperaturdifferenzen sehr präzise in Durchflüsse umgerechnet werden. Der hier verwendete Durchflussmesser SLG1430-320 ist für die Verwendung mit Wasser kalibriert.

In Abbildung 2.5 sind die Durchflusswerte beim Antrieb mit einer Spritzenpumpe und 


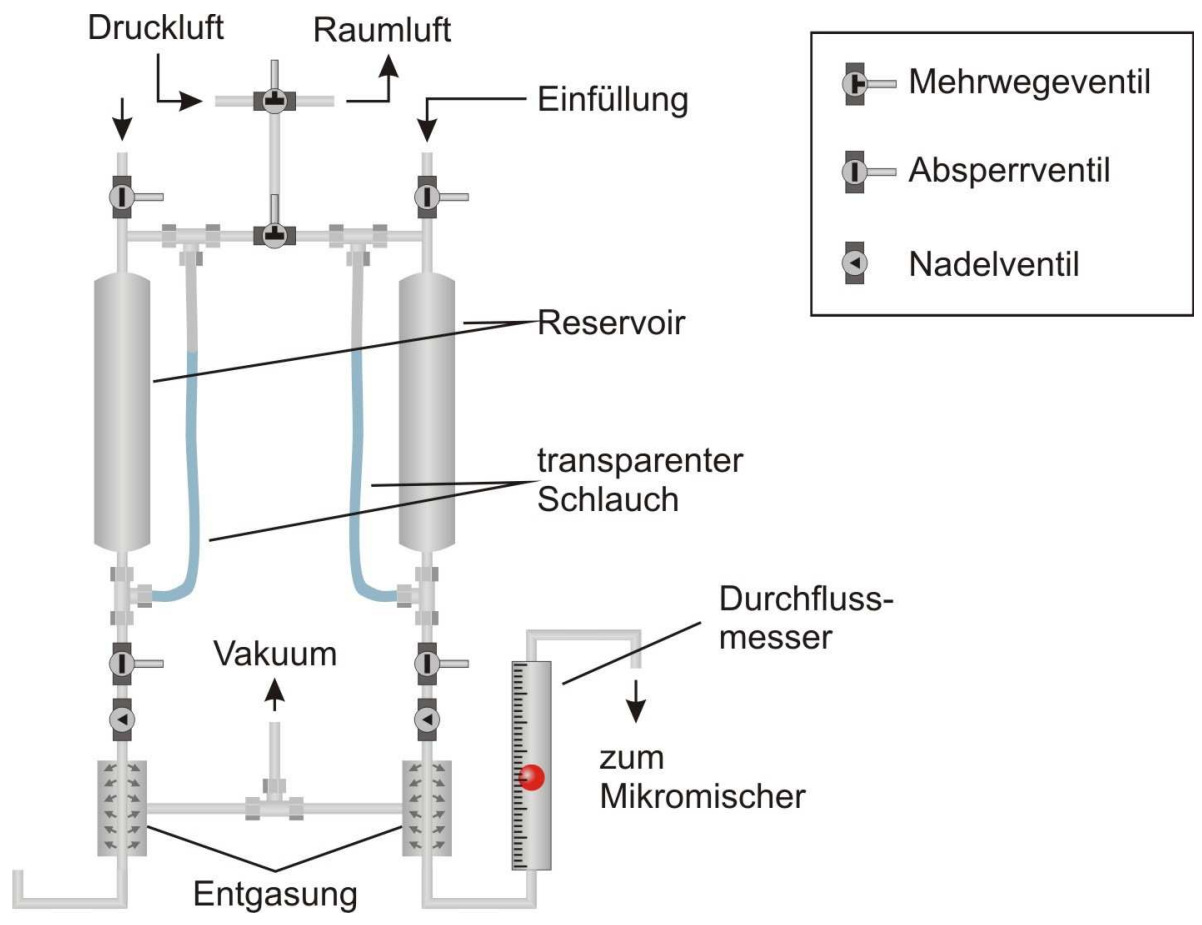

Abb. 2.6.: Flüssigkeitsantrieb durch Gasdruck

verschiedenen Spritzen verglichen. Zum Einsatz kamen 10 ml-Spritzen aus Kunststoff oder Glas. Die Kunststoffspritze und die Glasspritzen mit Glas- und Metallkolben sind handelsübliche Laborspritzen. Die letzte Glasspritze ist ein speziell gefertigtes Modell, bei dem darauf geachtet wurde, dass sich innerhalb der Spritze möglichst wenige Spannungen aufbauen können. Die Spritze stammt von der Firma „Innovative Labor Systeme“ (ILS). Zusätzlich ist noch eine Kurve eingetragen bei der die Strömung durch den Gravitationsdruck bei einem geringen Höhenunterschied zwischen Zu- und Auslauf angetrieben wurde. Bei allen Messungen wurde ein Wert von $1 \mu \mathrm{l} / \mathrm{min}$ eingestellt. Aufgrund der starken Schwankungen bei sämtlichen Spritzenmodellen ist dies nur bedingt genau möglich. Insgesamt schwanken die gemessenen Werte um bis zu $20 \%$. Eine präzise und konstante Einstellung ist mit keiner der verwendeten Spritzen möglich. Im Gegensatz dazu lässt sich durch die Änderung des Höhenunterschiedes eine sehr präzise Einstellung ohne jede Schwankung durchführen. Der Fehler liegt in diesem Fall bei etwa $1 \%$.

Die interessanten Durchflusswerte liegen beim zweiten Mikromischer um mehrere Größenordnungen höher. Ein wichtiger Grund für die hohen Werte ist der Verwendungszweck dieses Mischertyps. Das Forschungszentrum Karlsruhe entwickelt auf Grundlage der Multilamination eine Reihe von Mikromischern, die in chemischen Produktionprozessen eingesetzt werden sollen. Ein hoher Durchsatz ist hier ebenso erwünscht, wie eine effektive Mischung. Beide Forderungen werden durch hohe Durchflüsse unterstützt. Die Zielvorgaben erlauben es nicht, denselben Flüssigkeitsantrieb wie im ersten Fall zu ver- 
wenden. Dafür ist eine ebenso hohe Präzision bei der Einstellung der Durchflusswerte jedoch ebenfalls nicht notwendig. Verwendet wurde ein Antrieb durch Gasdruck, wie in Abbildung 2.6 gezeigt. Ein Gasdruckanschluss am oberen Ende der Flüssigkeitsreservoirs sorgt für einen kraftvollen Antrieb. Zur Kontrolle der Füllstände der Reservoirs ist ein transparentes Stück Schlauch parallel eingebaut. Reguliert wird der Durchfluss durch je zwei Ventile unterhalb der Reservoirs. Ein Absperrventil sorgt für einen schnellen Start oder Stopp des Flüssigkeitsstromes. Die genaue Einstellung übernehmen Nadelventile. Da Blasen während der Messungen sehr störend sind, werden die Flüssigkeiten in einem Membranschlauch durch starken Unterdruck zusätzlich entgast. Eine grundlegende Entgasung der Flüssigkeiten findet direkt vor der Befüllung der Reservoirs durch Erhitzen und Ultraschalleinwirkung statt. Ein Schwebekörper-Durchflussmesser bestimmt grob den eingestellten Volumenstrom. Die verwendeten Durchflussmesser sind nicht für eine genaue Messung geeignet. Eine genauere Durchflussmessung wurde dadurch realisiert, dass der Höhenunterschied der Flüssigkeiten im Reservoir am transparenten Schlauch abgelesen wurde. Aus dem daraus berechneten Volumen und der bekannten Durchflusszeit konnten genauere mittlere Durchflusswerte bestimmt werden. 


\section{2D-Molecular Tagging Velocimetry}

Eine der wichtigsten Informationen zur Analyse und Optimierung technischer Strömungssysteme ist das lokale Geschwindigkeitsfeld. Dynamische Vorgänge wie z.B. Mischprozesse lassen sich ohne die Kenntnis der zeitlichen Entwicklung des lokalen Strömungsfeldes nur schwer analysieren. Zumindest eine lokal begrenzte Kenntnis der Strömungsgeschwindigkeiten, bzw. des zeitlichen Strömungsverlaufs ist notwendig, um Schlüsse zu ziehen und Optimierungen vorzunehmen. Ein entsprechendes Interesse an der Beobachtung von Strömungen findet sich bereits bei Leonardo da Vinci [21]. Insbesondere für die Optimierung mikrofluidischer Mischungsvorgänge ist die Kenntnis des Strömungsfeldes unerlässlich. Zur Messung von Strömungsgeschwindigkeiten steht eine Vielzahl von Techniken zur Verfügung. Diese unterscheiden sich durch die angewandte Methodik und die Art der resultierenden Ergebnisse teilweise deutlich. Die Anzahl der erfassbaren Raumdimensionen reicht von punktweisen Messungen über weit verbreitete flächige Verfahren, bis hin zu aufwändigen dreidimensionalen Techniken. Unabhängig von der Zahl der Dimensionen, über die sich das gemessene Vektorfeld erstreckt, ist die Ahnzahl der messbaren Komponenten der vektoriellen Geschwindigkeiten. Im einfachsten Fall werden nur die Geschwindigkeitsbeträge bestimmt. Weit verbreitet sind Feldmessverfahren, die eine zweidimensionale Projektion der Vektoren in die Bildebene durchführen, daher können in diesen Fällen zwei Komponenten bestimmt werden. Speziellere Verfahren sind in der Lage alle drei Komponenten der vektoriellen Geschwindigkeiten zu bestimmen. Weniger gut räumlich auflösende Messverfahren besitzen häufig den Vorteil einer hohen Zeitauflösung. Im Folgenden werden vor allem zweidimensional ortsauflösende Messverfahren betrachtet. Entsprechend der angewandten Messmethode lassen sich die Verfahren aufteilen in partikelbasierte und solche, bei denen molekulare Tracer verwendet werden. Einen ausführlichen Überblick zu Geschwindigkeitsmessverfahren für die Mikrofluidik findet man in [65]. Ein Überblick, wie auch viele grundlegende theoretische Überlegungen zu mikrofluidischen Strömungen, findet sich außerdem in [2].

Bei den partikelbasierten Methoden sind als wichtigste Vertreter die Particle Image Velocimetry (PIV), die Particle Tracking Velocimetry (PTV) und die Laser Doppler Anemometrie (LDA, auch LDV für Laser Doppler Velocimetry) zu nennen. Das am weitesten verbreitete Messverfahren ist dabei die PIV. Wie der Name bereits sagt, werden dem Fluid Partikel zugesetzt, deren Position durch zwei, in kurzem zeitlichen Abstand aufeinander folgende Belichtungen, mit einer Kamera registriert wird. Die Bestimmung des, durch die Bewegung des Fluides verursachten, Partikelversatzes von einer Aufnahme zur nächsten geschieht anschließend mit einem Korrelationsalgorithmus und liefert eine vektorielle Darstellung des Strömungsfeldes. Das Verfahren wird bereits seit Jahrzehnten in den verschiedensten Bereichen eingesetzt und es existieren spezielle Modifikationen, durch die die PIV in nahezu jedem bekannten Strömungssystem genutzt werden kann. 


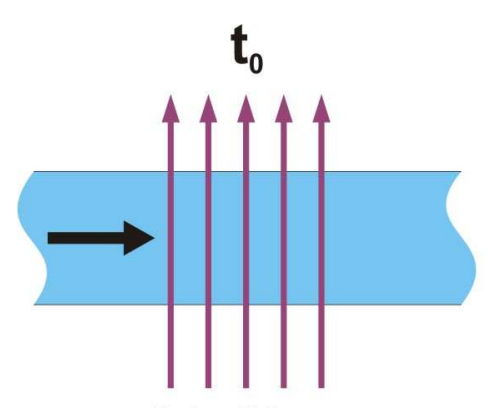

Schreiblaser

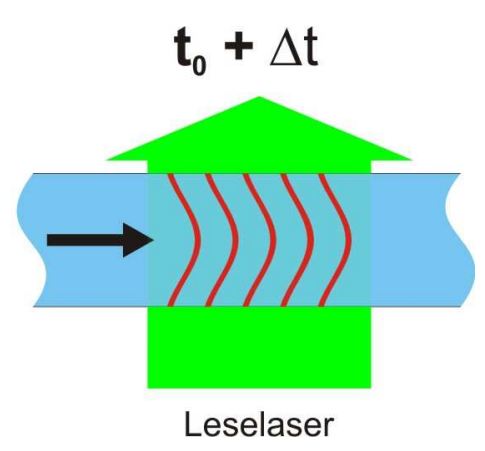

Leselaser

Abb. 3.1.: Prinzip der Geschwindigkeitsmessung mittels Molecular Tagging Velocimetry

Ein weiterer Bereich alternativer Messmethoden basiert auf der Nutzung molekularer Tracer. Im Wesentlichen können die wichtigsten Verfahren hier unter dem Begriff Molecular Tagging Velocimetry (MTV) zusammengefasst werden. Allen Verfahren gemein ist, dass sie keine Partikel nutzen, sondern sich der Eigenschaften molekularer Tracer bedienen. Die Markierungen werden in diesem Fall erzeugt, indem die Eigenschaften des Tracers oder des Fluides lokal durch eine strukturierte Beleuchtung verändert werden. An den belichteten Stellen treten dabei dauerhafte Veränderungen der Moleküleigenschaften auf, die sodann zeit- und ortsaufgelöst beobachtet werden können. Das Funktionsprinzip der MTV ist in Abbildung 3.1 gezeigt. Die zu untersuchende Strömung verläuft in diesem Fall von links nach rechts. Zum Zeitpunk $t_{0}$ wird, z.B. durch einen Laser, eine Struktur in das Fluid eingebracht. In der Abbildung ist das zum Beispiel ein Linienmuster. Dieser Prozess wird im Folgenden als „Schreibvorgang“ bezeichnet werden. Diese Struktur bewegt sich mit der Strömung. Sie kann durch Einsatz einer flächigen Detektion mit einer Kamera wiederholt ausgelesen werden (daher im Folgenden als „Lesevorgang“ bezeichnet), so dass aufeinander folgende Aufnahmen die Bewegung der Struktur mit der Strömung zeigen. Bei der Auswahl eines geeigneten Auswertungsalgorithmus stehen vor allem zwei wichtige Methoden zur Verfügung. Die korrelationsbasierten Algorithmen, wie sie bei der PIV verwendet werden und die so genannte "Methode des optischen Flusses".

Die Molecular Tagging Velocimetry bietet insbesondere den Vorteil, dass sie nicht auf Tracerpartikel angewiesen ist. Bei der Verwendung von Partikeln kann es Probleme geben, da die Eigenschaften der Partikel berücksichtigt werden müssen. So muss zum Beispiel die Dichte an die des umgebenden Fluides angepasst werden und die Größe der Partikel an die zu untersuchende Strömung. Bei elektrokinetischem Antrieb der Strömung muss darauf geachtet werden, dass die Partikel sich aufgrund statischer Aufladung nicht vollkommen anders verhalten als das Fluid. Zudem haben Partikel insbesondere in mikrofluidischen Systemen weitere Nachteile. Die Partikel müssen sehr klein werden um noch für eine Geschwindigkeitsmessung benutzt werden zu können. Dies reduziert aber die Signalausbeute erheblich. Größere Partikel könnten außerdem schmale Passagen verstopfen. Viele dieser Problematiken bestehen bei der Verwendung molekularer Tracer nicht mehr. Zudem ist man durch das Tagging in der Lage, beliebige Muster an 
jeder Stelle im Fluid zu erzeugen. Partikelbasierte Verfahren leiden unter bestimmten Bedingungen darunter, dass Strömungsbereiche wegen starker Wirbel partikelfrei bleiben, sich dort also keine Geschwindigkeiten messen lassen. In solchen Regionen mit starken Fliehkräften machen sich auch geringste Dichteunterschiede zwischen Fluid und Partikel bemerkbar. Im Bereich der Mikrofluidik treten aufgrund der langsamen laminaren Strömungen selten ausreichend große Kräfte auf, so dass sich dieses Problem dort nicht manifestiert. Zu beachten ist in kleinen Dimensionen dagegen der Einfluss der Diffusion. Bei partikelbasierten Verfahren ist in mikroskopischen Dimensionen durch die Brownsche Bewegung der Tracerpartikel eine deutliche Schwankung von Richtung und Betrag der gemessenen Geschwindigkeitsvektoren zu erwarten [25]. Bei den Tagging-Verfahren erlauben spezielle Algorithmen einen besseren Umgang mit der Diffusion der homogen im Fluid verteilten Farbstoffe.

Trotz gewisser Vorzüge ist die Anwendung von MTV-Verfahren insgesamt nicht sehr weit verbreitet. Dies liegt unter anderem daran, dass die Markierung der Strömung durch ein, mit einer strukturierten Beleuchtung, eingeschriebenes Muster wesentlich aufwändiger ist, als die Verwendung von Tracerpartikeln. In vielen Fällen wird dem Fluid daher nur eine einzelne, zur Hauptströmungsrichtung orthogonale, Linie eingeschrieben, über deren zeitliche Verformung die Entwicklung des Strömungsprofils beobachtet wird $[37,36,46,50,56]$. Noch seltener werden MTV-Verfahren für die zweidimensionale Vermessung von Strömungsfeldern genutzt. Insbesondere eine Vorgehensweise hat eine gewisse Verbreitung erreicht. Dabei wird ein Gittermuster in das Fluid geschrieben, das durch die Auffächerung eines Laserstrahls in viele Einzelstrahlen gebildet wird. Diese Variante kam in verschiedenen Systemen mit einigen Zentimetern Ausdehnung zum Einsatz, zum Beispiel zur Vermessung der Strömungsgeschwindigkeiten in der Gasphase im Modell eines Verbrennungsmotors [67]. Ebenfalls in der Gasphase wurde eine Düsenströmung vermessen [34]. In [59] wurde die Strömung von Wasser im Nachlauf einer koaxialen Düse untersucht. Der konvektive Flüssigkeitsstrom über einer gekühlten Platte wurde in [42] mit dem MTV-Verfahren untersucht. Eine umfangreichere Auflistung verschiedener Tagging-Techniken und Anwendungen findet sich in [30, 31, 33]. In [20] wird eine Überprüfung der MTV an einer einfachen Wirbelströmung durchgeführt. In [32] werden grundlegende Untersuchungen zu MTV in Kombination mit einer Visualisierungstechnik durch LIF vorgestellt.

In mikroskopisch kleinen Dimensionen wurde die zweidimensionale Variante der MTV dagegen bisher nicht eingesetzt. Einer der Gründe dafür ist, dass das Muster unter einem Winkel von $90^{\circ}$ zur Beobachtungsachse erzeugt wird. Dafür muss das zu untersuchende System seitlich optisch zugänglich sein. Bei vielen mikrofluidischen Systemen ist dies nicht oder nur mit sehr hohem Aufwand realisierbar. Das hier präsentierte Verfahren nutzt eine alternative Schreibtechnik, die die Erzeugung winziger Tagging-Muster ohne seitliche Transparenz des Untersuchungssystems ermöglicht. Weiterhin wurde anstatt der, auch beim MTV eingesetzten Korrelationsalgorithmen [19], ein alternativer Ansatz auf Grundlage der „Methode des optischen Flusses“ verwendet. Durch die geringen Mustergrößen ist der Einfluss der Diffusion auf die Messergebnisse nicht mehr zu vernachlässigen. Dieser Einfluss kann durch die angepassten Algorithmen kompensiert 
werden.

\subsection{Die Methode des optischen Flusses}

Die Auswertung der Bilddaten geschieht mit der so genannten „Methode des optischen Flusses". Grundlegende Einführungen in die verwendeten Algorithmen finden sich in $[1,22,28]$. Die Methode des optischen Flusses erlaubt zur Berechnung der Strömungsgeschwindigkeiten eine Anpassung an spezielle Gegebenheiten wie die Diffusion oder auch die Taylordispersion. Auf diese beiden Phänomene und die entsprechenden Anpassungen wird später noch genauer eingegangen. Weiterführende Details zu den speziellen Anpassungen sind zu finden in [16, 17]. Zunächst soll ein grober Überblick über die Funktionsweise der Methode und den Ablauf der Auswertung gegeben werden.

Der optische Fluss entspricht dem Vektorfeld, das die Verzerrungen beschreibt, die notwendig sind, um ein Bild in ein anderes zu verwandeln. Wenn man Aufnahmen einer markierten Strömung zugrunde legt, dann entspricht der optische Fluss dem Vektorfeld der Strömungsgeschwindigkeiten, das zur Verwandlung der ursprünglichen Aufnahme in die folgenden notwendig ist. Für die Bestimmung des optischen Flusses wird als grundlegende Voraussetzung die Erhaltung der Grauwerte $I$ im Verlauf der Bildsequenz gefordert:

$$
\begin{aligned}
\frac{d I}{d t} & =\frac{\partial x}{\partial t} \frac{\partial I}{\partial x}+\frac{\partial y}{\partial t} \frac{\partial I}{\partial y}+\frac{\partial I}{\partial t} \\
& =u \frac{\partial I}{\partial x}+v \frac{\partial I}{\partial y}+\frac{\partial I}{\partial t}=0
\end{aligned}
$$

Diese Gleichung kann auch in einer Vektorschreibweise angegeben werden, die Datenund Parameterterme voneinander trennt:

$$
\frac{d I}{d t}=\mathbf{d}^{\top} \cdot \mathbf{p}=\left[\frac{\partial I}{\partial x} \frac{\partial I}{\partial y} \frac{\partial I}{\partial t}\right] \cdot\left[\begin{array}{lll}
u & v & 1
\end{array}\right]^{\top}=0
$$

Mit dieser Gleichung lassen sich nun zwar die Datenterme, also die örtlichen und zeitlichen Gradienten des Grauwertes aus den Aufnahmen berechnen, eine Lösung der Gleichung ist aber nicht möglich. Da nur eine Gleichung mit zwei Unbekannten vorliegt ist das System unterbestimmt. Zur Lösung ist eine weitere Annahme notwendig. In diesem Fall wird von der Strömung in der lokalen Umgebung eines Punktes Glattheit gefordert. Das bedeutet, dass dieselbe Differentialgleichung im Prinzip auch für die benachbarten Bildpunkte gelten soll. Solange die Umgebung relativ klein bleibt kann davon ausgegangen werden, dass diese Annahme gültig ist. Übliche Umgebungen sind zum Beispiel $11 \times 11$ Bildpunkte groß. Man erhält ein System von $n=121$ Gleichungen mit unterschiedlichen Datentermen $\mathbf{d}_{i}$ aber denselben Parametern p. Auch dieses Gleichungssystem kann 
wieder in vektorieller Schreibweise angegeben werden:

$$
\mathbf{W D} \cdot \mathbf{p}=\mathbf{W}\left[\begin{array}{ccc}
\frac{\partial I_{1}}{\partial x} & \frac{\partial I_{1}}{\partial y} & \frac{\partial I_{1}}{\partial t} \\
\frac{\partial I_{2}}{\partial x} & \frac{\partial I_{2}}{\partial y} & \frac{\partial I_{2}}{\partial t} \\
\vdots & \vdots & \vdots \\
\frac{\partial I_{n}}{\partial x} & \frac{\partial I_{n}}{\partial y} & \frac{\partial I_{n}}{\partial t}
\end{array}\right] \cdot\left[\begin{array}{l}
u \\
v \\
1
\end{array}\right]=0
$$

Die Datenterme $\mathbf{d}_{i}$ wurde zur $n \times 3$ Datenmatrix $\mathbf{D}$ zusammengefasst. Zusätzlich ist hier die $n \times n$ Gewichtungsmatrix $\mathbf{W}$ eingeführt worden. Die Matrix enthält eine zweidimensionale Gaußfunktion, die um den mittlere Bildpunkt zentriert ist. Sie reduziert den Einfluss der Bildpunkte am Rand der betrachteten Nachbarschaft und gewichtet die inneren stärker, die sich in der Nähe des Zentrums befinden. Damit erhält der Bereich in der Nähe des Bildpunktes, dem später die berechnete Geschwindigkeit zugeordnet wird, einen höheren Einfluss.

Das überbestimmte Gleichungssystem kann nun gelöst werden. Die Gradienten im Datenterm $\partial I_{1} / \partial x, \partial I_{1} / \partial y$ und $\partial I_{1} / \partial t$ können durch einen optimierten Sobelfilter [62] aus den Intensitätsaufnahmen bestimmt werden. Der Filter benötigt je nach Größe auch in Zeitrichtung mehrere Bildpunkte, bzw. Aufnahmen. Üblich ist ein Sobelfilter mit einer Kantenlänge von fünf, daher werden mindestens fünf aufeinander folgende Aufnahmen für die Berechnung benötigt. Für die beiden ersten Aufnahmen können auf diese Weise keine Geschwindigkeiten berechnet werden, da nicht genügend Daten vorliegen. Ebenso werden bei der Berechnung die zwei äußersten Bildpunkte an den Rändern einer Aufnahme verworfen.

Nachdem der Datenterm konkret bestimmt wurde, muss das Gleichungssystem gelöst werden. Hier kommt ein gewichteter „total least squares“ Ansatz zum Einsatz. Um den Parameterterm p zu bestimmen, muss eine Analyse des Eigensystems der Matrix $\mathbf{J}=$ $\mathbf{W}^{\top} \mathbf{D}^{\top} \mathbf{D W}$ mit $\mathbf{J} \in \mathbb{R}^{5 \times 5}$ durchgeführt werden. Der Parametervektor $\mathbf{p}$ ist nun der normalisierte Eigenvektor zum kleinsten Eigenwert. Die Matrix J ist auch bekannt als Strukturtensor. Die Parameter werden durch den Strukturtensor für jeden Bildpunkt einzeln berechnet.

\subsubsection{Diffusion und Taylordispersion}

Bei der Formulierung der Algorithmen wurden bisher wichtige physikalische Vorgänge vernachlässigt, die sich auf die Intensitätsverteilungen in den Aufnahmen auswirken und dazu führen, dass die Annahme eines konstanten Grauwertes verletzt wird. Die Diffusion der Farbstoffmoleküle verwischt mit fortschreitender Zeit den ursprünglich steilen Intensitätsgradienten und sorgt für ein Abflachen der Gradienten. Ein weiteres Problem entsteht durch die so genannte Taylordispersion [69, 70]. Zwischen der Deck- und Bodenplatte fließt das Fluid aufgrund der laminaren Strömung mit einem parabelförmigen Strömungsprofil. Dieser Schverhalt ist in Abbildung 3.2 dargestellt. Eine schmale Säule stellt einen Bereich höherer Intensität zum Zeitpunt $t_{1}$ dar, der über die Tiefe des Kanals reicht. Dieser würde durch die Strömung ebenfalls parabelförmig verformt. Eine Aufnahme der Intensitäten bei Blickrichtung von oben würde die verformte Säule in die 


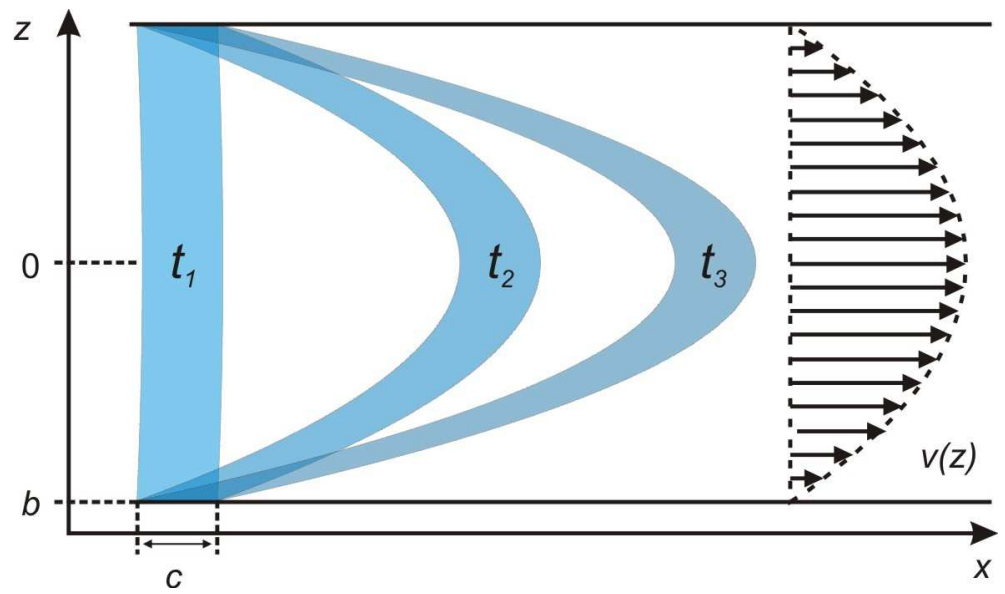

Abb. 3.2.: Parabelförmiges Strömungsprofil zwischen Deck- und Bodenplatte

Bildebene projizieren und damit die Intensitätsverteilung in der Tiefe des Kanals integrieren. Dadurch wird die Intensitätsverteilung zu späteren Zeiten $t_{2}$, bzw. $t_{3}$ langsam verwischt und es entsteht durch die Abbildung eine Art Schweif (siehe auch Abbildung 3.14). Die zu späteren Zeitpunkten registrierte lokale Intensität stammt - je nachdem welche Position im Bild betrachtet wird - aus unterschiedlicher Tiefe. Damit variiert auch die zugehörige berechnete Geschwindigkeit mit der Position im Bild. Das Verwischen der ursprünglichen Fluoreszenzintensitätsverteilung durch das parabelförmige Strömungsprofil bezeichnet man auch als Taylordispersion.

Durch die sehr kleinen Abmessungen und die im Experiment verwendeten geringen Strömungsgeschwindigkeiten liegen die Reynoldszahlen der untersuchten Strömungen im einstelligen Bereich und darunter. Man kann also von einer laminaren Strömung eines newtonschen Fluides ausgehen. Bei newtonschen Fluiden bleibt die Viskosität konstant, wenn sich die Scherkräfte ändern, die auf das Fluid wirken. Das parabelförmige Geschwindigkeitsprofil einer solchen Strömung ist nach Hagen-Poisseuille gegeben durch

$$
u(z)=\frac{a}{2} z^{2}-a \cdot b \cdot z \quad \text { mit } \quad a=\frac{1}{\mu} \frac{d P}{d x}, \frac{d P}{d x} \leq 0,
$$

dabei ist $2 b$ der Abstand zwischen Deck- und Bodenplatte, $\mu$ ist die Viskosität des Fluides und $d P / d x$ ist der Druckgradient entlang der Strömung.

Das Muster wird, wie in Abbildung 3.2 gezeigt, zum Zeitpunkt $t_{0}$ in das Fluid geschrieben. Wie später gezeigt werden wird (Abbildung 3.3), ist die Absorption des Farbstoffs vernachlässigbar klein, so dass das Muster mit gleichmässiger Intensität entlang der gesamten Tiefe geschrieben wird. Eine zunächst säulenförmige Markierung wird mit der Zeit durch die Strömung verformt. Abbildung 3.2 zeigt die Verteilung des Farbstoffes auch zu späteren Zeitpunkten. Die Markierung ist nun entsprechend des parabelförmigen Geschwindigkeitsprofils verformt. Da die Kamera durch eine der Deckplatten die 
Fluoreszenz aus der gesamten Kanaltiefe registriert, wird die Intensität über die Tiefe integriert. Die aufgezeichnete Intensität wird durch das folgende Integral reproduziert:

$$
I=\int_{b \pm \sqrt{b^{2}+\frac{2 x}{a t}}}^{b \pm \sqrt{b^{2}+\frac{2(x+c)}{a t}}} 1 d z=\sqrt{\left\|b^{2}+\frac{2 \cdot(x+c)}{a \cdot t}\right\|}-\sqrt{\left\|b^{2}+\frac{2 \cdot x}{a \cdot t}\right\|}
$$

Die Integrationsgrenzen wurden durch eine zeitliche Integration von Gleichung 3.4 und Auflösen nach z bestimmt. I gibt dann die aufgenommene Intensität wieder und t entspricht der Zeit, die seit dem Tagging-Vorgang bei $t_{0}$ vergangen ist. $\mathrm{x}$ ist die räumliche Koordinate in Strömungsrichtung. Die Funktion kann durch eine Taylor-Entwicklung um Null approximiert werden:

$$
I=\sqrt{\frac{2}{t}}\left(\sqrt{\frac{x+c}{a}}-\sqrt{\frac{x}{a}}\right)+\frac{b^{2} \sqrt{t}}{s \sqrt{2}}\left(\sqrt{\frac{a}{x+c}}-\sqrt{\frac{a}{x}}\right)+\mathcal{O}\left(t^{\frac{3}{2}}\right)
$$

Eine zeitliche Ableitung des ersten Terms führt auf die folgende Differentialgleichung.

$$
\frac{d I}{d t}=\frac{d}{d t}\left(\sqrt{\frac{2}{t}}\left(\sqrt{\frac{x+c}{a}}-\sqrt{\frac{x}{a}}\right)\right)=-\frac{1}{2 t} I
$$

Die Kombination mit Gleichung 3.1 führt zu einer erweiterten Differentialgleichung zur Beschreibung des Vorgangs:

$$
\frac{d I}{d t}=u \frac{\partial I}{\partial x}+v \frac{\partial I}{\partial y}+\frac{\partial I}{\partial t}=-\frac{1}{2 t} I
$$

Hier sind $\mathrm{u}$ und $\mathrm{v}$ die Komponenten des zweidimensionalen Geschwindigkeitsvektors in Richtung x und y. Die Gleichung kann wiederum in vektorieller Schreibweise angegeben werden.

$$
\frac{d I}{d t}=\mathbf{d}^{\top} \cdot \mathbf{p}=\left[\frac{1}{2 t} I \frac{\partial I}{\partial x} \frac{\partial I}{\partial y} \frac{\partial I}{\partial t}\right] \cdot\left[\begin{array}{llll}
1 & u & v & 1
\end{array}\right]^{\top}=0
$$

Wie zuvor ist dabei $\mathbf{d}$ der Datenvektor und $\mathbf{p}$ der Parametervektor. In dieser Gleichung ist die Diffusion noch nicht berücksichtigt. Es ist aber einfach möglich, auch diese Erweiterung aufzunehmen. Dazu wird die Differentialgleichung für einfache isotrope Diffusion $d I / d t=D \Delta I$, mit $\Delta I=\partial^{2} I / \partial x^{2}+\partial^{2} I / \partial y^{2}$ verwendet.

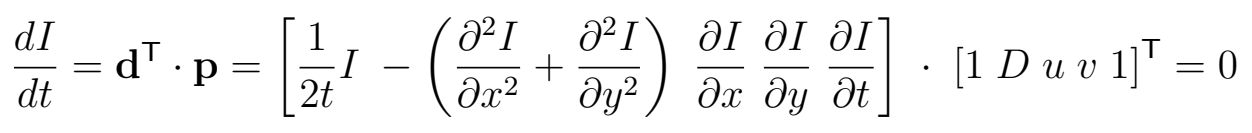

$D$ ist die Diffusionskonstante des Fluides. In diesem Fall wird eine zweidimensionale isotrope Diffusion angenommen. Diese Annahme ist eine starke Vereinfachung, lässt sich aber kaum umgehen, da die dritte Komponente $\partial^{2} I / \partial z^{2}$ nicht direkt bestimmt werden kann. Mögliche Annahmen unter Zuhilfenahme der ursprünglichen Intensität führen zu sehr komplexen Gleichungen, die nur schwer zu lösen sind. Problematisch ist die Annahme 
zweidimensionaler Diffusion vor allem für längere Zeiträume. Im Falle relativ kurzer Zeiten verglichen mit dem Zeitraum, in dem die Diffusion für nennenswerte Änderungen sorgt, kann aber erfolgreich mit dieser Annahme gearbeitet werden.

Gleichgung 3.10 enthält eine Formulierung, die mit der Diffusion und der Taylordispersion die beiden wichtigsten in mikrofluidischen Strömungen relevanten Vorgänge umfasst. Es ist nun möglich, die resultierenden Strömungsgeschwindigkeiten mit dem Algorithmus zu berechnen. Die Gradienten werden dabei wiederum durch einen SobelFilter berechnet, ebenso geschieht die Lösung des Gleichungssystems weiterhin durch den Strukturtensoransatz.

Es sind weitere Anpassungen des Algorithmus möglich, zum Beispiel können lineare Helligkeitsänderungen in die Gleichungen aufgenommen werden, so dass es im betrachteten Zeitraum zum Beispiel zu Schwankungen der Anregungsenergien kommen darf. Zu berücksichtigen ist dabei, dass jeder unbekannte Parameter eine Ausweitung der Nachbarschaftsregion erfordert um eine präzise Abschätzung der Unbekannten zu ermöglichen. Idealerweise sollte die Zahl der unbekannten Parameter also so gering wie möglich gehalten werden.

\subsection{Mustererzeugung}

Zur Berechnung von Strömungsgeschwindigkeiten benötigt der vorgestellte Algorithmus Aufnahmen von Intensitätsverteilungen. Es müssen dabei Muster vorhanden sein, die der Strömung folgen. Im Gegensatz zu weit verbreiteten Verfahren wie PIV oder PTV werden zu diesem Zweck bei der MTV keine Partikel eingesetzt, sondern ein molekularer Tracer. Die Eigenschaften des molekularen Tracers ermöglichen eine Markierung (engl. tagging, daher der Name „Molecular Tagging Velocimetry“) des strömenden Fluides. Es gibt verschiedene Möglichkeiten, um ein Muster durch Veränderung der molekularen Struktur des Tracers in der Strömung zu verankern. Die folgenden Prozesse können im Sinne des „Tagging“" genutzt werden.

- Fluoreszenzaktivierung

- Photobleaching

- Phosphoreszenz

- Farbänderung

- Temperaturänderung

Abgesehen von der Temperaturänderung benötigen alle Verfahren einen geeigneten molekularen Tracer. Für eine Temperaturänderung kann das Fluid selbst als Tracer verwendet werden.

Mit „Fluoreszenzaktivierung“ ist in diesem Fall ein Prozess gemeint, bei dem ein ursprünglich nicht fluoreszierender Farbstoff so verändert wird, dass er anschließend in der Lage ist, zu fluoreszieren. Ein so genannter „caged dye" ist ein solcher Farbstoff. 
Im Prinzip besteht das Farbstoffmolekül aus einem gewöhnlichen Farbstoffmolekül und einer zusätzlichen funktionalen Gruppe. Diese zusätzliche Gruppe unterdrückt die Fluoreszenz des Farbstoffmoleküls. Die Farbstoffmoleküle sind also auch nach elektronischer Anregung mit Laserlicht zunächst nicht in der Lage, die aufgenommene Energie als Fluoreszenzlicht wieder abzugeben. Ein intensiver UV-Puls ist in der Lage, die kovalente Bindung der funktionalen Gruppe mittels Photolyse aufzubrechen und den ursprünglichen Farbstoff freizusetzen. Dieser kann dann wieder mit einem weiteren Laserpuls zur Fluoreszenz angeregt werden. Das Ergebnis ist eine lokal erhöhte Fluoreszenzintensität an Orten, an denen im „caged dye“ zuvor mittels UV-Bestrahlung ein Bindungsbruch initiiert wurde [18]. Für dieses Verfahren ist ein speziell modifizierter und daher sehr teurer Farbstoff notwendig. Die Anwendung kann somit äußerst kostspielig werden.

Beim Photobleaching nutzt man den umgekehrten Weg und sorgt für lokal verringerte Fluoreszenzintensitäten. Dies geschieht, indem durch intensive Laserbestrahlung ein möglichst großer Anteil der Farbstoffmoleküle zerstört (photolysiert) wird [50]. Prinzipiell kann für dieses Vorgehen jeder gewöhnliche Fluoreszenzfarbstoff verwendet werden. Ausreichend große Intensitätsunterschiede sind bei diesem Verfahren jedoch unter Umständen schwierig zu erzeugen.

Im Fall der Phosphoreszenz nutzt man langlebige Energiezustände der Farbstoffe, die nach der Anregung über einen langen Zeitraum Licht emittieren [46]. Es muss darauf geachtet werden, dass einerseits lang genug Licht emittiert wird, um eine Messung durchführen zu können, andererseits aber die Intensität des emittierten Lichts zur Detektion ausreicht.

Eine Farbänderung der Fluoreszenz bestimmter Farbstoffe lässt sich bei Einstrahlung von UV-Licht erreichen. Mit passenden Filtersätzen wird die Farbänderung als HellDunkel Unterschied wahrgenommen $[20,75]$.

Die letzte genannte Möglichkeit der Markierung eines Fluides ist eine Temperaturänderung [10]. Dabei wird das Fluid lokal erwärmt und die Abstrahlung dieser wärmeren Zonen mit einer IR-Kamera detektiert. Diese Methode benötigt zwar für gewöhnlich als einzige keinen Tracer, ist jedoch am schwierigsten durchzuführen, da Tagging und Detektion im mittleren Infrarot (zum Beispiel bei $3 \mu \mathrm{m}$ ) stattfinden. Dafür sind spezielle Laser und Kameras notwendig, deren Kosten bei weitem über denen gleichwertiger Systeme im sichtbaren Bereich liegen. Außerdem ist die Wärmediffusion sehr hoch, verglichen mit der Diffusion von Farbstoffmolekülen. Damit werden die Markierungen sehr schnell unleserlich.

Von den beschriebenen Möglichkeiten wurde in der hier vorliegenden Arbeit die Verwendung eines „caged dyes“ gewählt. Vorteile dieser Technik sind eine relative hohe Intensitätsausbeute und eine vergleichsweise gute Verfügbarkeit. Es stehen mehrere Farbstoffe mit unterschiedlichen spektralen Eigenschaften zur Verfügung, so dass eine Farbstoffvariante gefunden werden konnte, die mit den vorhandenen Lasern und Kameras optimal verwendet werden konnte. Zusätzlich sind die kommerziell verfügbaren Farbstoffe an ein relativ großes Dextran-Molekül gebunden, so dass die Diffusion der Farbstoffe stark reduziert wird. 


\subsection{Caged dyes}

Verschiedene Eigenschaften des Farbstoffes müssen bei der Anwendung des Verfahrens beachtet werden. Verwendet wurde „Caged Carboxy-Q-Rhodamin Dextran“, das unter der Bestellnummer D-34678 bei der Fa. Molecular Probes erhältlich ist. Informationen zur Herstellung und zu einfachen Eigenschaften des Farbstoffs werden in [18] präsentiert. Die Basis des Farbstoffes ist ein Rhodamin. Die spektralen Eigenschaften entsprechen denen des reinen Rhodamin, das Absorptionsmaximum liegt laut Herstellerangaben bei $545 \mathrm{~nm}$ und das Emissionsmaximum der Fluoreszenz liegt bei $575 \mathrm{~nm}$. Ein großer Nachteil molekularer Tracer ist ihre geringe Größe, da die Diffusion für kleine Moleküle wesentlich schneller voranschreitet als für große. Um diesem Sachverhalt entgegen zu wirken, werden die Farbstoffmoleküle an Dextrane gebunden. Diese sind erheblich größer, so dass auch die Diffusionsgeschwindigkeit reduziert wird. An ein Dextran-Molekül sind je nach Größe mehrere Farbstoffmoleküle gebunden. Im Mittel haben die verwendeten Moleküle ein Molekulargewicht von 10.000 Dalton.

\subsubsection{Spektrale Eigenschaften}

Die Wellenlänge des Anregungslasers und die spektrale Empfindlichkeit des Detektionssystems müssen an die spektralen Eigenschaften des Farbstoffes angepasst werden. Dazu ist die genaue Kenntnis des Absorptionsspektrums des Farbstoffes notwendig. Darum wurde zunächst das Absorptionsspektrum im relevanten Spektralbereich zwischen 300 und $800 \mathrm{~nm}$ mit einem Spektrometer (UV/VIS/NIR-Spektrometer Lambda 19, Fa. Perkin Elmer) vermessen. Der Farbstoff wurde dazu mit einer Konzentration von 500 mg/l in demineralisiertem Wasser gelöst und die Lösung in eine Küvette gefüllt. Die Konzentration ist dieselbe, die später für die mikrofluidischen Untersuchungen verwendet wurde. Die Küvette besteht aus Quarzglas und ist damit im gesamten Bereich zu nahezu $100 \%$ transparent, hat also keinen Einfluss auf die Absorptionsmessung. Außerdem kann davon ausgegangen werden, dass das Wasser keinen nennenswerten Beitrag zur Absorption leistet. Das aufgenommene Spektrum beruht also allein auf den Eigenschaften des gelösten Farbstoffs.

Die Tiefe der Küvette und damit die im Medium zurückgelegte Strecke beträgt $10 \mathrm{~mm}$. Bei den Strömungsmessungen liegt die Schichtdicke der Kanäle und Kammern dagegen nur bei $200 \mu \mathrm{m}$. Die im Medium zurückgelegte Strecke des Lichts ist aufgrund schrägen Lichteinfalls mit ca. $300 \mu \mathrm{m}$ etwas länger. Die gemessenen Absorptionswerte wurden durch das Lambert-Beersche Gesetz auf die, im Experiment relevante, Stecke korrigiert. Das Ergebnis ist in Abbildung 3.3 dargestellt. Die Vergrößerung zeigt, dass im Bereich zwischen 500 und $530 \mathrm{~nm}$ ein Maximum der Absorption liegt. Dies ist auch in etwa der Bereich, der laut Herstellerangabe (um $545 \mathrm{~nm}$ ) ideal für die Anregung der Fluoreszenz ist. Außerdem ist ersichtlich, dass bei den Schichtdicken der Mikrokanäle nur ein sehr geringer Anteil des Laserlichts vom Versuchsfluid absorbiert wird. Der Energieeintrag ist folglich äußerst gering und die Auswirkung auf die Strömungsmessung zu vernachlässigen. Im Bereich der UV-Wellenlängen steigt die Absorption zwar noch erheblich stärker an, diese Wellenlängen können für die Fluoreszenzanregung aber nicht verwendet werden, 


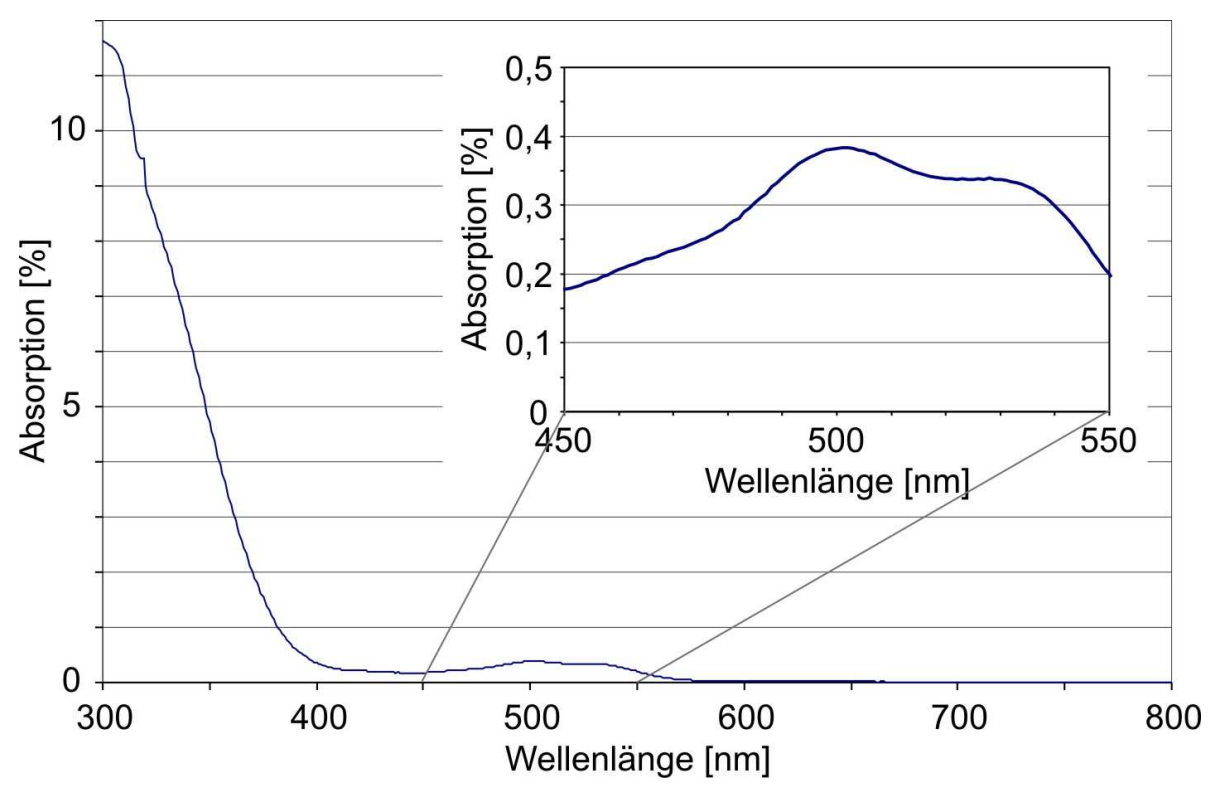

Abb. 3.3.: Spektraler Verlauf der Absorption des caged dyes

da sie, wie beim Schreibvorgang, für einen Bindungsbruch und damit zu einer weiteren Freisetzung von Fluoreszenzfarbstoff sorgen. Beim Auslesen des Musters darf dieses aber verständlicherweise nicht gleichzeitig verändert werden.

\subsubsection{Kinetik des Bindungsbruchs}

In der Literatur sind bisher kaum Daten zur Kinetik des Photolyseschritts bei Bestrahlung des Farbstoffs mit UV-Licht verfügbar. Es ist bekannt, dass der Bindungsbruch nicht instantan mit dem eingestrahlten Laserpuls erfolgt, sondern erst nach geraumer Zeit nach dem initiierenden Laserpuls abgeschlossen ist. Daher liegt das in die Strömung geschriebene Muster erst nach einer gewissen Zeit in voller Intensität vor. Zur genauen Bestimmung der zeitlichen Entwicklung wurde eine einfache Untersuchung vorgenommen. Die Intensität der Fluoreszenz der Farbstoffösung wurde mit einer schnellen Kamera zeitaufgelöst aufgenommen. Für die Fluoreszenzaufnahmen wurde eine Kamera (Fa. Andor, Ixon) bei 8fachem Pixelbinning betrieben, um eine Bildrate von ca. $138 \mathrm{~Hz} z u$ erreichen. Für die Aufnahmen wurde der Farbstoff in den Mikrokanal eingebracht und anschließend die Strömung gestoppt. Ein UV-Laserpuls eines XeF-Excimerlasers (Compex 150T, Fa Lambda Physik) bei einer Wellenlänge von $351 \mathrm{~nm}$ sorgte für die Freisetzung des Farbstoffes und ein kontinuierlich eingestrahlender Argon-Ionen-Laser (Innova 300, Fa. Coherent) bei einer Wellenlänge von $514 \mathrm{~nm}$ sorgte für eine Fluoreszenzanregung der freigesetzten Farbstoffmoleküle. Die zeitliche Entwicklung der Fluoreszenzintensität wurde von der Kamera vor und nach dem Schreibvorgang aufgezeichnet. Die Intensitäten wurden örtlich über den vom UV-Laser belichteten Bereich des Fluides gemittelt, so dass 


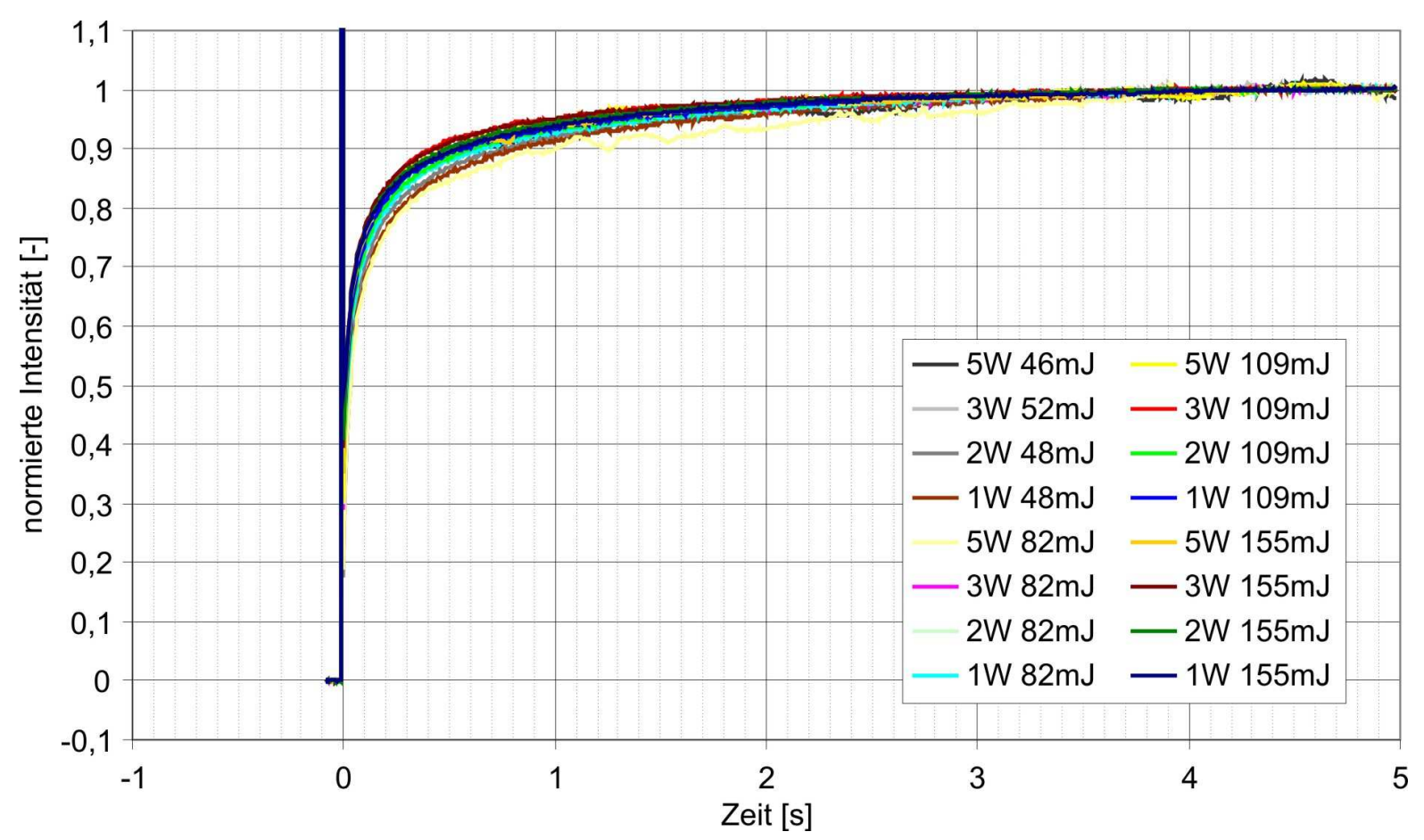

Abb. 3.4.: Intensitätsentwicklung der Fluoreszenz nach Photolyse des caged dyes bei verschiedenen Energien des Schreibpulses und des Leselasers. Für alle Kurven wurde der Hintergrundwert, der vor dem Schreibpuls registriert wurde, abgezogen und es wurde auf das Plateau zwischen 4 und 5 Sekunden normiert.

sich ein einzelner mittlerer Intensitätswert zu jedem Zeitpunkt ergab. Die resultierenden Intensitätsentwicklungen sind in Abbildung $3.4 \mathrm{zu}$ sehen.

Die Kurven unterscheiden sich in den Pulsenergieen, die für den Photolyse-Schritt verwendet wurden, und in der Leistung des Lasers, der zum Auslesen der Fluoreszenz verwendet wurde. Beide Einstellungen wurden variiert, um einen Einfluss auf den Verlauf der Reaktion untersuchen zu können. Der UV-Puls ist jeweils bei $t_{0}$ vorzufinden. Damit der Verlauf der Kurven trotz unterschiedlicher Einstellungen und damit resultierender Intensitäten verglichen werden konnte, wurden zunächst die Hintergrundintensitäten abgezogen, die vor dem Schreibvorgang bei $t_{0}$ registriert wurden. Die Intensitäten vor dem Schreibvorgang liegen auch vor dem Schreibvorgang nicht bei Null, da auch unter günstigen Bedingungen immer ein wenig freigesetzter Farbstoff existiert. Somit ist in jedem Fall Fluoreszenzlicht detektierbar. Nach Abzug dieser Intensitäten liegen alle Werte vor $\mathrm{t}_{0}$ bei Null, wie man in Abbildung 3.4 deutlich erkennen kann. Im nächsten Schritt werden die Kurven auf das Plateau normiert, das sich im Verlauf der Messungen ausbildet. Normiert wurde jeweils auf den Mittelwert der Werte am Ende der Messung zwischen $\mathrm{t}=4 \mathrm{~s}$ und $\mathrm{t}=5 \mathrm{~s}$. Die Kurven laufen nun alle in denselben Wert aus.

Nach dieser Angleichung kann man den Verlauf der Kurven vergleichen und Aussagen zur zeitlichen Entwicklung der Intensitäten machen. Die x-Achse wurde in ein zehntel- 
Sekunden-Raster eingeteilt, dies entspricht genau der Aufnahmefrequenz von $10 \mathrm{~Hz}$, die bei den späteren Geschwindigkeitsmessungen verwendet wurde. Die Kurven unterscheiden sich in ihrem Verlauf kaum. Die Unterschiede von maximal $5 \%$ zeigen keine Korrelation zu den eingestellten Laserenergien, bzw. -leistungen. Leichte Schwankungen, wie sie im Verlauf einzelner Kurven auftreten ergeben sich durch Intensitätsschwankungen des Anregungslasers. Man erkennt, dass bei einer Messung mit $10 \mathrm{~Hz}$ bereits bei der ersten Aufnahme nach dem Schreibpuls ein großer Teil des Farbstoffs freigesetzt wurde. $\mathrm{Zu}$ diesem Zeitpunkt betragen die Intensitäten etwa $70 \%$ des Maximalwertes. Es kann also davon ausgegangen werden, dass das geschriebene Muster bereits auf der ersten Aufnahme nach dem Schreibpuls gut erkennbar vorliegt. Für schnellere Strömungen, bei denen das Zeitfenster von 100 ms zwischen zwei Aufnahmen zu lang ist, bietet sich die Verwendung eines caged dyes nicht an. Der weitere Verlauf der Intensitätsentwicklung ist langsamer. Das Maximum wird erst ab ca. 3 s erreicht. Bei der Anwendung der Algorithmen ist zu beachten, dass es durch Intensitätsänderungen zu Fehlern kommen kann. Eine lineare Änderung der Intensitäten mit der Zeit wird bei der Auswertung berücksichtigt. Für kurze Zeitintervalle von fünf Aufnahmen bei einer Aufnahmefrequenz von $10 \mathrm{~Hz}$, wie sie bei den Untersuchungen verwendet wurden, trifft diese Annahme immer besser zu, je weiter man sich von den ersten Aufnahmen entfernt. Insbesondere bei Einbeziehung der ersten Aufnahmen kann es zu größeren Fehlern kommen, da der Intensitätsverlauf deutlich von der Annahme eines linearen Verlaufs abweicht.

\subsubsection{Ausbleichen des Farbstoffs}

Neben der steigenden Fluoreszenzintensität, durch die Photolyse nach dem Schreibvorgang, sorgt Photobleaching für eine Abnahme der Intensitäten. Photobleaching bedeutet eine irreversible Zerstörung der fluoreszierenden Moleküle, die in diesem Fall durch den Leselaser, der die Fluoreszenz anregt, begründet ist. Um die Auswirkungen des Bleaching auf die resultierenden Intensitäten und den zeitlichen Verlauf des Prozesses zu untersuchen, wurden die Intensitätsänderungen der Fluoreszenz bei längerer Bestrahlung überprüft. Verwendet wurde dazu eine Farbstofflösung, bei der bereits ein großer Anteil des Farbstoffes von vorn herein freigesetzt vorliegt. Dadurch ist ein Schreibpuls nicht notwendig. Die Entwicklung der Intensitäten ist in Abbildung $3.5 \mathrm{zu}$ finden.

Im Verlauf der Bestrahlung nimmt die Intensität zwar sichtbar ab, der Unterschied beträgt aber nicht einmal $1 \%$ über die gesamte Messdauer. Außerdem ist die Dauer dieser Messung bei weitem höher als bei den späteren Geschwindigkeitsmessungen (23 s). Die Auswirkung des Bleaching auf die gemessenen Intensitäten, und damit die berechneten Geschwindigkeiten, ist also zu vernachlässigen.

\subsubsection{Sättigung der Intensitäten}

Die unterschiedlichen Optimierungsschritte bei der Erstellung des experimentellen Aufbaus führten unter anderem dazu, dass der ursprünglich zur Fluoreszenzanregung genutzte frequenzverdoppelte Nd:YAG-Laser bei $532 \mathrm{~nm}$ durch einen kontinuierlich betriebenen Argon-Ionen-Laser bei einer Wellenlänge von 514 nm ersetzt wurde. Die Pulsenergie des 


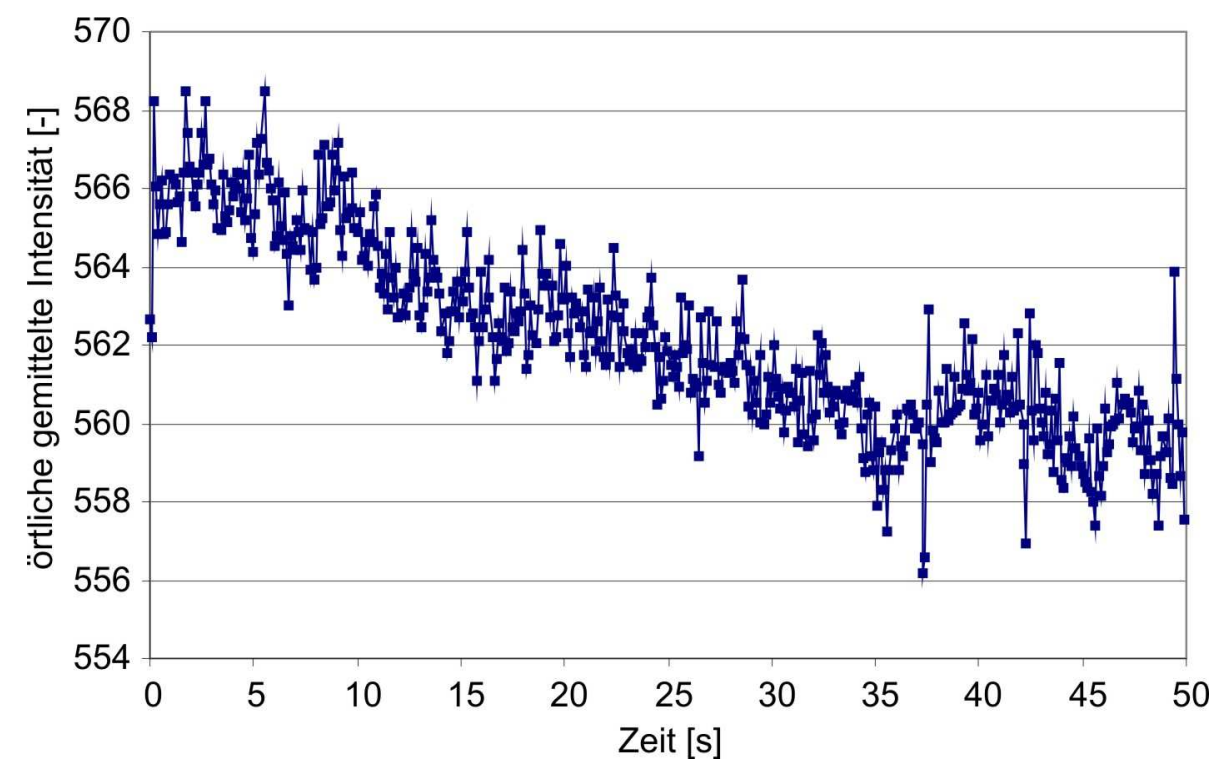

Abb. 3.5.: Intensitätsminderung durch Photobleaching bei kontinuierlicher Bestrahlung

Nd:YAG-Lasers beträgt maximal $200 \mathrm{~mJ}$ und die Energie, die der Argon-Ionen-Laser während der Belichtungszeit von 10 ms einstrahlt, liegt bei maximal 90 mJ. Trotzdem sind die aufgenommenen Fluoreszenzintensitäten bei Verwendung des cw-Lasers bei weitem höher. Dies lässt darauf schließen, dass es ab einer bestimmten Leistungsdichte zu einer Sättigung der Fluoreszenzausbeute kommt, bei der eine weitere Erhöhung der eingestrahlten Energie keinen Sinn mehr macht. Im Folgenden soll dies sowohl für die Energie des Schreibpulses als auch für die Anregung der Fluoreszenz beim Auslesen überprüft werden.

Für die Messungen wurde wiederum das Vorgehen verwendet, das auch in den letzten Abschnitten schon zum Einsatz kam. Im Mikrokanal befand sich bei abgestellter Strömung die Farbstofflösung. Der Schreibpuls setzte Farbstoff frei und die Fluoreszenzintensitäten bei Anregung mit dem Argon-Ionen-Laser wurden mit einer Kamera aufgenommen. Anschließend wurde die mittlere Intensität zu einem definierten Zeitpunkt nach dem Schreibvorgang bestimmt. In Abbildung 3.6 sind die Intensitäten aufgetragen, die sich bei unterschiedlichen Einstellungen des Leselasers ergeben. Der Schreibpuls hatte bei diesen Messungen jeweils die maximal mögliche Energie von $210 \mathrm{~mJ}$. Es ist deutlich zu erkennen, dass die Intensitäten ab einer Leistung des Leselasers von etwa $3 \mathrm{~W}$ nicht mehr weiter steigen. Im zweiten Fall wurde die Leistung des Leselasers auf $1 \mathrm{~W}$ festgelegt und die Energie des Schreibpulses variiert. Abbildung 3.7 zeigt die Entwicklung der Intensitäten bei unterschiedlich starken Schreibpulsen. Auch in diesem Fall ist deutlich zu erkennen, dass die Intensitäten einem Maximalwert entgegen streben. Dieser wird aufgrund der begrentzten Energie des Lasers aber nicht erreicht.

Aufgrund dieser Messungen ist einzusehen, dass ein kontinuierlich betriebener Laser 


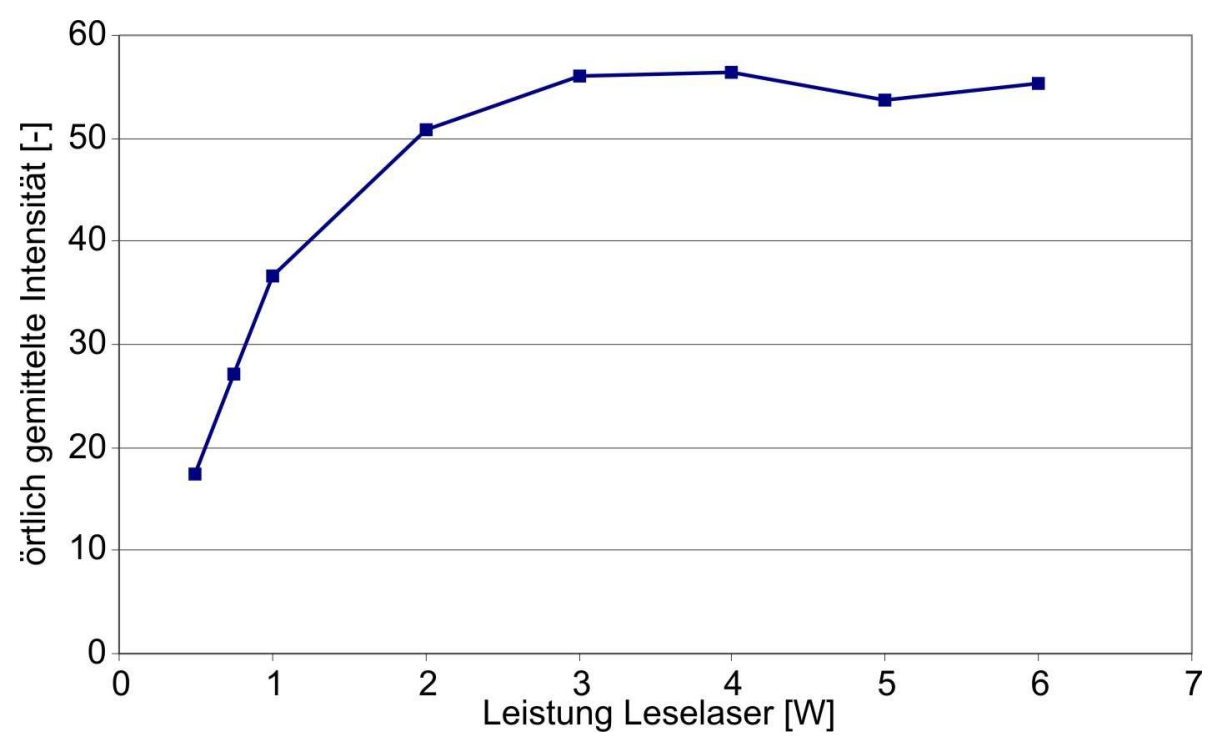

Abb. 3.6.: Intensitätsentwicklung bei steigender Leistung des Leselasers, Energie des Schreibpulses bei allen Messungen $210 \mathrm{~mJ}$

hinsichtlich der Intensitätsausbeute der Fluoreszenz einem gepulsten deutlich überlegen ist. Der entscheidende Unterschied sind die Zeiten in denen die Laser ihre Energien abgeben. Der kontinuierliche Laser kann jedes Fluorophor aufgrund der sehr kurzen Lebensdauer der Fluoreszenz von wenigen ns mehrfach anregen, da die Energie im Verlauf der Belichtungszeit von $10 \mathrm{~ms}$ abgegeben wird. Der gepulste Laser dagegen emittiert seine gesamte Pulsenergie im Verlauf von wenigen ns. Damit kann jedes Fluorophor nur wenige Male angeregt werden. Wenn selbst ein kontinuierlich betriebener Laser, wie in Abbildung 3.6 gezeigt, schon die Sättigung der maximal möglichen Intensitäten überschreitet, dann wird diese Schwelle im Fall des kurzen Laserpulses bei weitem überschritten worden sein, so dass nur ein geringer Teil der Strahlung tatsächlich zur Anregung der Farbstoffmoleküle beigetragen hat.

Durch die Sättigung ergeben sich Grenzwerte für die Energie der verwendeten Laserstrahlung. Entscheidend für das Erreichen einer Sättigung ist ein bestimmter Energiewert pro Zeit und Fläche, also eine Leistungsdichte. Um eine gesteigerte Intensitätsausbeute zu erreichen ohne die Grenzwerte der Leistungsdichte zu überschreiten, können alle drei Werte, also Energie, Zeit und Fläche angepasst werden. Eine Verteilung der Energie auf eine größere Fläche ermöglicht den Einsatz höherer Energien. Im Fall eines vorgegebenen Mikrosystems ist die Fläche allerdings nicht veränderbar. Auch eine Verteilung der Energie auf einen längeren Zeitraum ist nur begrenzt möglich, da die Detektion mit möglichst kurzer Belichtungszeit durchgeführt werden müssen, um ein Verwischen der Strukturen zu verhindern. Eine Steigerung der detektierten Intensitätswerte ist durch die verwendeten Laser also nur bedingt möglich.

Grenzwerte für die eingestrahlte Laserenergie existieren nicht nur aufgrund der festge- 


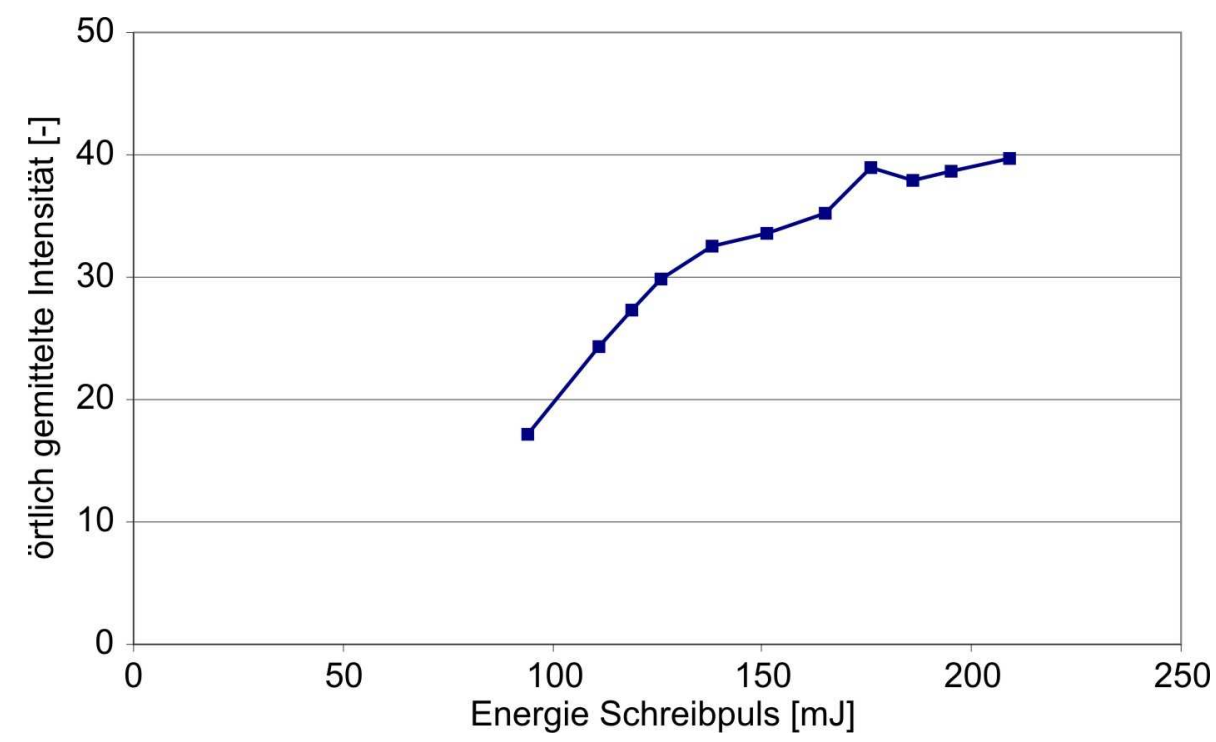

Abb. 3.7.: Intensitätsentwicklung bei steigender Energie des Schreibpulses, Leistung des Leselasers bei allen Messungen $1 \mathrm{~W}$

stellten Sättigung, sondern auch durch die Zerstörschwellen der verwendeten Materialien und weil der Energieeintrag in das Fluid nicht zu hoch ausfallen sollte. Ein hoher Energieeintrag könnte die zu untersuchende Strömung durch induzierte konvektive Strömungen aufgrund der entstehenden Wärme verändern. Dieser Vorgang wäre bei einer Messung unerwünscht, da die Messtechnik keinen Einfluss auf das Messergebnis haben darf.

\subsection{Experimenteller Aufbau}

Die Erweiterung der konventionellen MTV zu einem zweidimensionalen Geschwindigkeitsmessverfahren für die Mikrofluidik erfordert einige Anpassungen. Insbesondere der Schreibvorgang muss so modifiziert werden, dass die Erzeugung eines zweidimensionalen Musters möglich ist. Dabei ist zu beachten, dass viele mikrofluidische Systeme von der Seite her nicht optisch zugänglich sind.

Der verwendete Aufbau, wie in Abbildung 3.8 gezeigt, kann in drei wesentliche Bereiche eingeteilt werden, die fluidischen Komponenten, die Mustererzeugung und die Detektion. Das Untersuchungsobjekt, hier ein Mikromischer, befindet sich im Zentrum des Aufbaus. Er ist so montiert, dass er durch Mikrometertriebe in allen drei Raumrichtungen präzise verfahren werden kann. Bei der Entwicklung des Geschwindigkeitsmessverfahrens wurde das modulare Mikromfluidiksystem eingesetzt, das in Kapitel 2.1 vorgestellt wurde. In diesem System ist es möglich, auf einfache Weise verschiedene Strömungsgeometrien zu realisieren. Im Verlauf der Messungen wurde neben dem Mikromischer auch ein gerader Kanal eingesetzt. Der Strömungsantrieb kann unterschiedlich realisiert werden. In Kapitel 2.3 wurden die verschiedenen Möglichkeiten dargestellt. Im abgebildeten Fall wurde 


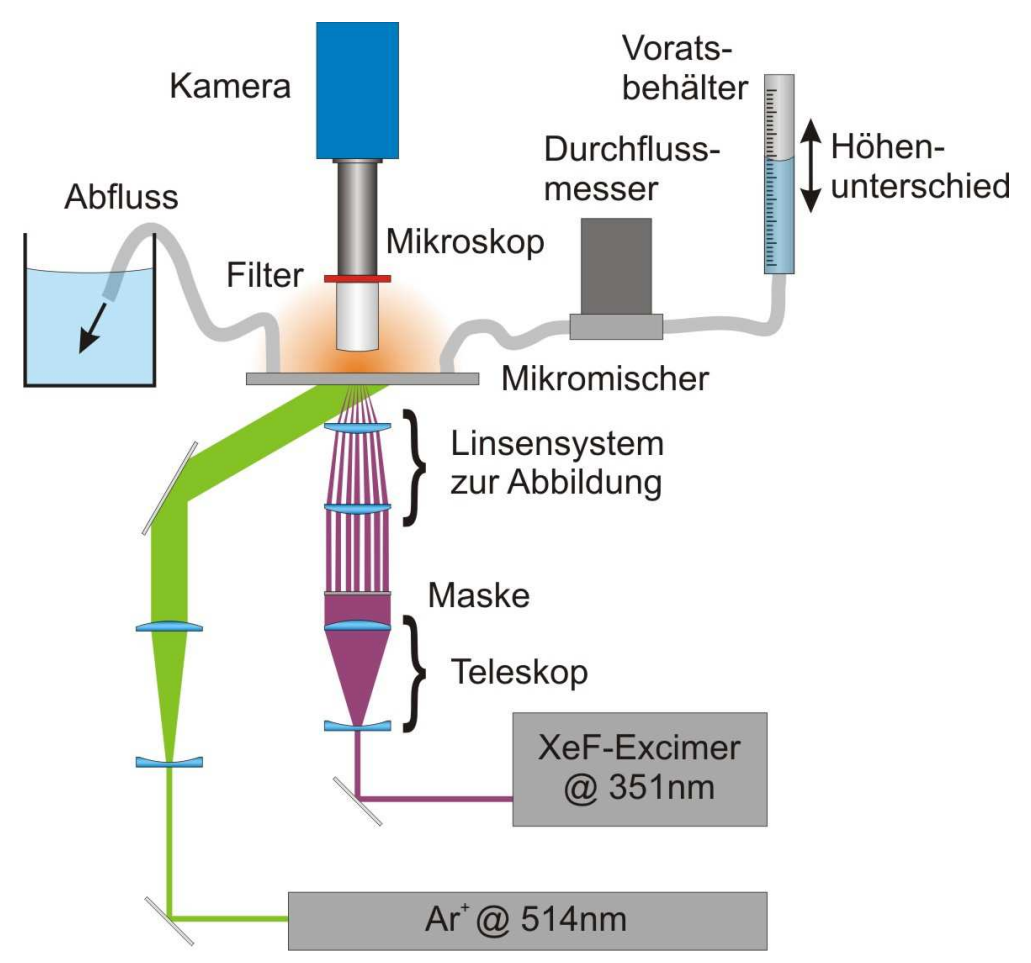

Abb. 3.8.: Experimenteller Aufbau zur Erfassung von 2D-MTV Sequenzen

für den Antrieb der Höhenunterschied zwischen den Reservoirs des Zu- und Ablaufs genutzt. Der aktuelle Volumenstrom wurde durch einen Durchflussmesser aufgezeichnet. Die Werte wurden synchron zur Bildaufnahme der Geschwindigkeitsmesstechnik registriert, um später einen Vergleich zu ermöglichen. Als Versuchsfluid wurde demineralisiertes Wasser verwendet, da es ungiftig und einfach verfügbar ist. Für die Geschwindigkeitsmessung wurde zusätzlich ein Farbstoff benötigt, der als Tracer eine optische Markierung der Strömung ermöglicht. Zu diesem Zweck wurde im Wasser der beschriebene „caged dye" in einer geringen Konzentration von $500 \mathrm{mg} / \mathrm{l}$ gelöst. Mit ihm ist es möglich, ein fluoreszierendes Muster in der Flüssigkeit zu generieren. Der Schreibvorgang geschieht örtlich strukturiert durch eine Maskenabbildung. Dadurch ist es möglich, ein definiertes Muster in der Flüssigkeit zu verankern.

Die Mustererzeugung wird durch eine Maskenabbildung durchgeführt. Der Strahl eines XeF-Excimer Lasers (Compex 150T, Fa Lambda Physik) bei $351 \mathrm{~nm}$ wird aufgeweitet, um eine Maske vollständig auszuleuchten. Diese Maske wird durch ein Linsensystem scharf auf das zu untersuchende Objekt abgebildet. Das Linsensystem besteht aus einer großen Konvexlinse $(75 \mathrm{~mm}, \mathrm{f}=210 \mathrm{~mm})$, die eine Vorverkleinerung vornimmt und einer kleineren, die die eigentliche Abbildung durchführt (50 mm, $\mathrm{f}=60 \mathrm{~mm})$. Die erste Linse sorgt dafür, dass die zweite Linse möglichst nur im inneren Bereich ausgeleuchtet wird, wodurch die Abbildungsfehler aufgrund sphärischer Abberation minimiert werden. Das Linsensystem verkleinert die jeweilige Maske etwa um den Faktor sieben. Die Maske darf 
maximal $40 \times 40 \mathrm{~mm}$ groß sein und wird in einem fest positionierten Halter fixiert. Im Mikrosystem kann durch dieses System eine Fläche von maximal 5,7 × 5,7 mm markiert werden. Kleinere Bereiche lassen sich durch entsprechend kleinere Masken mit feineren Strukturen markieren. Problematisch kann unter diesen Umständen die Energie des Schreiblasers werden. Für eine gleich bleibende Intensität in den späteren Aufnahmen muss dabei dieselbe Laserenergie für den Schreibprozess auf kleinerer Fläche verwendet werden. Dies ist zwar durch eine entsprechende Strahlformung möglich und aus Sicht der Masken ebenfalls machbar, führt aber zu sehr hohen Leistungsdichten der Laserstrahlung im Messvolumen. In diesem Fall ist zu beachten, dass die Zerstörschwellen der verwendeten Materialien nicht überschritten werden dürfen.

Die Auswahl einer geeigneten Maske hängt vor allem vom untersuchten Objekt ab. Das Muster der Maske muss außerdem für den verwendeten Algorithmus optimiert werden. Auf diesen Punkt soll später noch genauer eingegangen werden. Die Masken können auf verschiedene Art gefertigt werden. Die Muster können dabei entweder auf ein transparentes Material gedruckt werden oder aus einem nicht-transparenten Material ausgeschnitten werden. Gedruckte Masken haben den Vorteil, dass auch Grauwerte aufgrund unterschiedlich transparenter Bereiche realisierbar sind. Ein Nachteil ist, dass die Farben durch die hohen Leistungsdichten des verwendeten Lasers ablatiert werden können. Im Fall großer Untersuchungsobjekte wurden zunächst auch relativ große Masken verwendet. Dazu wurde der Laser aufgeweitet, weshalb die Leistungsdichten vergleichsweise gering waren. In diesem Fall konnten gedruckte Masken verwendet werden. Die Masken wurden mit einem Grafikprogramm erstellt und auf transparenten Kunststofffolien ausgedruckt. Ein Vorteil dieser Methode ist, dass sich nahezu jede beliebige Maske sehr schnell herstellen lässt. Nachteilig ist dagegen, dass die zur Belichtung eingesetzte UV-Strahlung relativ stark von den Folien absorbiert wird. Es geht dabei ca. ein Drittel der Laserenergie durch die Absorption des Folienmaterials verloren. Spätere Messungen wurden in kleinere Systemen von nur $1 \mathrm{~mm}$ Querschnitt durchgeführt. In diesem Fall muss der Laser fokussiert werden und die Leistungsdichten am Ort der Maske werden zu hoch für bedruckte Folien. Ein weiteres Problem ist, dass die Druckerfarben nicht in der Lage sind schwarze Bereiche ausreichend undurchlässig zu erzeugen. Die Masken sind also auch in dunklen Bereichen ein wenig transparent, so dass in den zugehörigen Bereichen im Fluid ebenfalls Farbstoff freigesetzt wird. Auf die Auswahl bestimmter Muster der Maske wird im folgenden Abschnitt 3.4.1 detaillierter eingegangen.

Nachdem der Schreibvorgang abgeschlossen ist, kann das Muster jederzeit ausgelesen werden. In diesem Fall wird die zu untersuchende Flüssigkeitsströmung mit einem ArgonIonen Laser (Innova 300, Fa. Coherent) bei einer Wellenlänge von $514 \mathrm{~nm}$ vollständig ausgeleuchtet und das Fluoreszenzlicht des Farbstoffes mit einer Kamera aufgenommen. Das unerwünschte Anregungslicht wird durch einen Filter (OG570, Fa. Schott) unterdrückt. Die Kamera (Imager Compact, Fa. LaVision) nimmt mit einer festen Frequenz von $10 \mathrm{~Hz}$ Bilder der Intensitätsverteilung der Fluoreszenz des Farbstoffes auf. Die Belichtungszeit der Kamera ist mit $10 \mathrm{~ms}$ relativ lang, damit die registrierten Intensitäten möglichst hoch sind. Durch die kontinuierliche Anregung der Fluoreszenz führen längere Belichtungszeiten direkt zu höheren aufgezeichneten Intensitäten. Die Belichtungszei- 

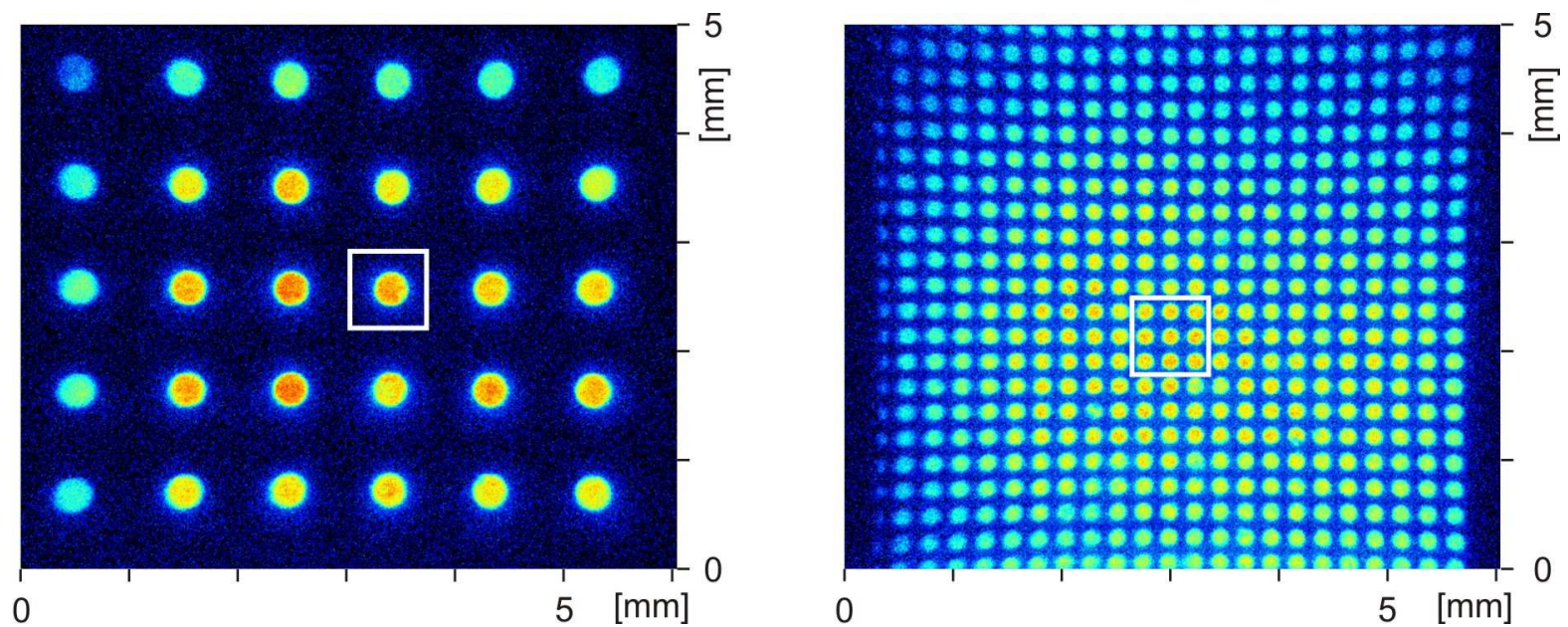

Abb. 3.9.: Taggingmuster kurz nach dem Schreibvorgang, links: langsame Strömung rechts: schnelle Strömung

ten dürfen dabei niemals so lang sein, dass es zu einer sichtbaren Verschmierung einer bewegten Struktur während einer einzelnen Belichtung kommt.

\subsubsection{Masken und Muster}

Die Auswertealgorithmen müssen so gut wie möglich an die experimentellen Bedingungen angepasst werden. Genauso gilt dies aber auch umgekehrt: Die Generierung von MTVBildsequenzen muss möglichst gut an die verwendeten Algorithmen angepasst werden. Insbesondere das Muster, welches in das Fluid geschrieben wird, kann nahezu beliebig verändert werden. Naheliegende Muster sind zum Beispiel Linien, Gitter oder Punkte. In den meisten Fällen wird beim Tagging das Linien-Tagging verwendet und aus der Verformung der Linie das tangentiale Strömungsprofil bestimmt. In einigen Fällen wurden für zweidimensionale Messungen bereits Gittermuster eingesetzt. Die verwendeten Verfahren setzen aber voraus, dass die Untersuchungssysteme seitlich optisch zugänglich sind.

Problematisch ist bei der Auswertung mit der Methode des optischen Flusses, dass der Algorithmus nicht in der Lage ist, Geschwindigkeitswerte parallel zu einem Intensitätsgradienten zu bestimmen. Das bedeutet, dass eine Linie immer nur die Geschwindigkeitskomponenten senkrecht zu ihrer Ausdehnung anzeigen könnte, nicht aber diejenige entlang der Linie. Punktmuster sind daher Linienmustern vorzuziehen. Wenn die Punkte möglichst klein sind, verglichen mit den auftretenden Strömungsstrukturen, stehen immer zwei Seiten dem Fluss tangential gegenüber. Das Muster lässt sich also über die Punktgröße an die Strömung anpassen. Ein weiterer Parameter eines Punktmusters ist die Dichte der Punktverteilung. Prinzipiell ist anzunehmen, dass man ein dichtes Vektorfeld mit einer möglichst dichten Verteilung erhält. Da ein weniger dichtes Muster weniger 


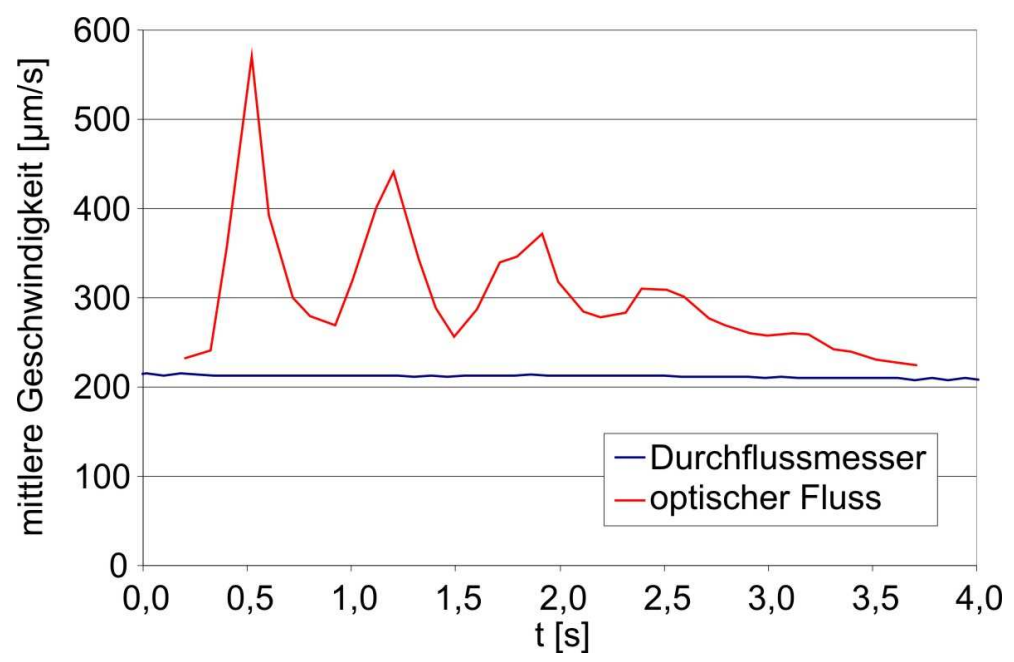

Abb. 3.10.: Mittlere Strömungsgeschwindigkeiten bei schneller Strömung (Abbildung 3.9 links)

Bereiche mit Intensitätsgradienten aufweist, ergäbe sich auch ein weniger dichtes Vektorfeld.

In Abbildung 3.9 sind zwei mögliche Punktmuster nach dem Taggingvorgang abgebildet. Die Aufnahmen wurden der Einfachheit halber in einem relativ breiten $(5 \mathrm{~mm})$ Kanal gemacht. Die Strömung verläuft jeweils von links nach rechts. Jede der beiden Aufnahmen steht für eine Sequenz, die im Folgenden ausgewertet und analysiert wurde. Im Fall der linken Abbildung sind die Punkte sehr viel weiter verteilt als bei der rechten. Außerdem ist die zugrunde liegende Strömung mit $0,5 \mathrm{ml} / \mathrm{h}$ nur halb so schnell eingestellt wie bei der rechten mit $1 \mathrm{ml} / \mathrm{h}$. Die Sequenzen wurden, wie im Abschnitt 3.1 beschrieben, ausgewertet und die resultierenden Vektorfelder wurden jeweils räumlich gemittelt. Die Randbereiche oben und unten wurden dabei ausgeschlossen. Die Vektoren im Inneren des Kanals sollten ein Plateau gleich hoher Geschwindigkeiten ausbilden, da die Strömung laminar verläuft. Die Mittelung glättet in diesem Fall also lediglich fehlerhaft berechnete Geschwindigkeiten. Die mittleren Strömungsgeschwindigkeiten wurden in den Abbildungen 3.10 und 3.11 aufgetragen (rote Linien). Verglichen wurden sie jeweils mit den gleichzeitig aufgenommenen Werten des Durchflussmessers (blaue Linien). Beide Messungen stimmen zunächst gut mit denen des Durchflussmessers überein. Nach kurzer Zeit schon weichen die Messwerte im Fall der schnellen Strömung (Abbildung 3.10) erheblich nach oben ab. Nachdem ein Maximalwert erreicht wurde, sinken die gemessenen Geschwindigkeiten wieder und steigen nach einer Weile erneut an. Dieses Verhalten wiederholt sich ein Paar mal. Im Fall der langsameren Strömung (Abbildung 3.11) ist deutlich länger keine Abweichung zu erkennen. Erst nach 1,3 Sekunden steigen die Werte leicht an. Die Abweichungen sind nicht plausibel durch die Strömung zu erklären, da keine Änderung an den Einstellungen erfolgte. Die gleichzeitigen Messungen des Durchflussmessers haben ebenfalls gezeigt, dass die Strömung gleichmäßig verläuft und keine 


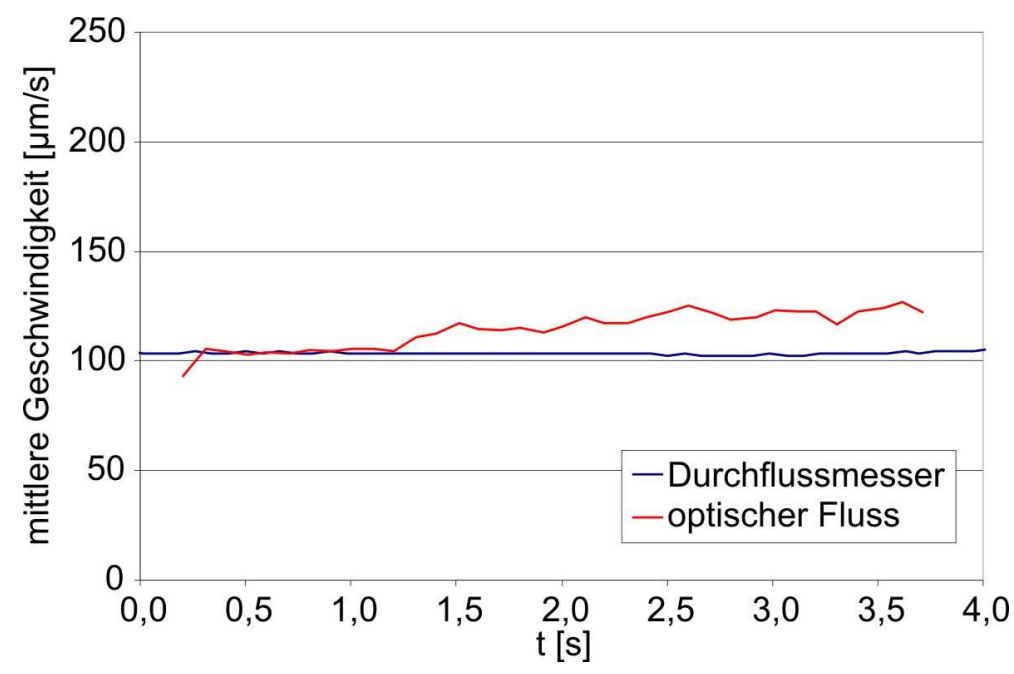

Abb. 3.11.: Mittlere Strömungsgeschwindigkeiten bei langsamer Strömung (Abbildung 3.9 rechts)

Pulsationen aufweist.

Interessant ist der Vergleich der berechneten Geschwindigkeitswerte mit den zugehörigen Bildern der gemessenen Fluoreszenzintensität. Für den Vergleich wurden die markierten Ausschnitte aus Abbildung 3.9 vergrößert und im zeitlichen Ablauf dargestellt. Abbildung 3.12 und 3.13 zeigen die entsprechenden Bildserien. Abgebildet ist nur jede zweite Aufnahme. Der wichtigste Unterschied zwischen den beiden Sequenzen ist, dass die Punkte in Abbildung 3.12 ineinander laufen, während in Abbildung 3.13 der nächstliegende Punkt nicht einmal den vergrößerten Bereich erreicht. Wenn man sich die Abbildungen 3.10 und 3.12 genauer ansieht fällt auf, dass die maximal überhöhte Geschwindigkeit bei $t=0,6$ s gerade zu dem Zeitpunkt auftritt, zu dem die jeweils nächste Punktmarkierung die Position der davor liegenden erreicht. Die ursprüngliche Position zweier Punkte ist in Abbildung 3.12 jeweils oben rechts mit zwei weißen Kreisen gekennzeichnet. Auch bei $\mathrm{t}=1,2 \mathrm{~s}$ und $\mathrm{t}=1,8 \mathrm{~s}$ treffen wiederum die nächsten Punkte an den ursprünglichen Positionen der davor liegenden Markierungen ein. Wiederum stimmen diese Zeitpunkte mit den maximalen Strömungsgeschwindigkeiten überein, die die Auswertung ergab.

Der Grund für die falsche Geschwindigkeitsberechnung ist also höchstwahrscheinlich das Ineinanderlaufen der markierten Bereiche. Sobald eine Markierung mit einer anderen überlappt, steigt die lokale Fluoreszenzintensität. Dies ist eine Verletzung der Annahmen des konstanten Grauwertes, wie sie im Abschnitt 3.1 für den Algorithmus getroffen wurden. Sobald ein Punkt die Region des davor liegenden erreicht, steigt die Intensität und die berechneten Geschwindigkeiten steigen dadurch ebenfalls. Danach ändert sich der Grauwert eine Weile kaum noch, daher nähern sich die berechneten Geschwindigkeiten wieder den korrekten Werten an, bis der nächste Punkt erreicht wird. Auf diese Weise entsteht die periodisch überhöhte Geschwindigkeit in Abbildung 3.10. Da der Fall 


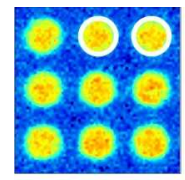

$0,0 \mathrm{~s}$

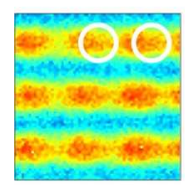

$1,0 \mathrm{~s}$

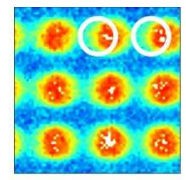

$0,2 \mathrm{~s}$

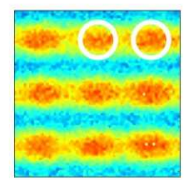

$1,2 \mathrm{~s}$

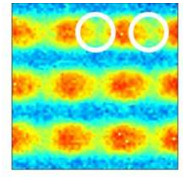

$0,4 \mathrm{~s}$

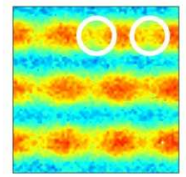

$1,4 s$

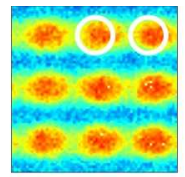

$0,6 s$

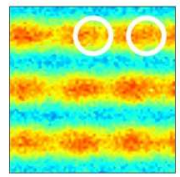

$1,6 s$

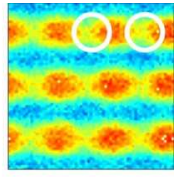

$0,8 \mathrm{~s}$

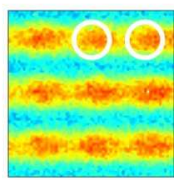

$1,8 \mathrm{~s}$

Abb. 3.12.: Zeitliche Sequenz des Taggingmusters der schnellen Strömung aus Abbildung 3.9 links

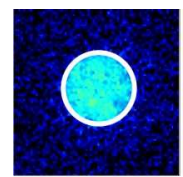

0,0 s

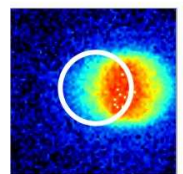

$1,0 \mathrm{~s}$

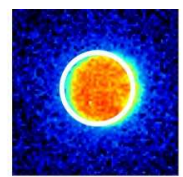

$0,2 \mathrm{~s}$

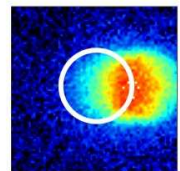

$1,2 \mathrm{~s}$

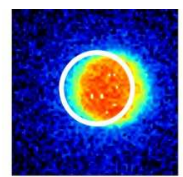

$0,4 \mathrm{~s}$

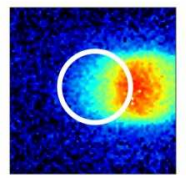

$1,4 s$

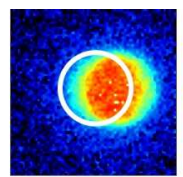

$0,6 s$

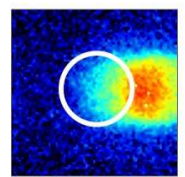

$1,6 s$

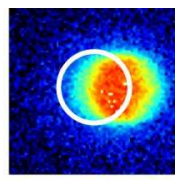

$0,8 \mathrm{~s}$

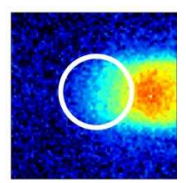

$1,8 \mathrm{~s}$

Abb. 3.13.: Zeitliche Sequenz des Taggingmusters der langsamen Strömung aus Abbildung 3.9 rechts

des Ineinanderlaufens der Markierungen durch den Algorithmus nicht erfasst wird, muss verhindert werden, dass dies während einer Aufnahmesequenz geschieht. Das Punktmuster muss also in seiner Dichte an die jeweilige Strömung angepasst werden. Dabei ist es auch möglich, bereits vorliegende Informationen über das Strömungsfeld zu nutzen und die Muster nur in Regionen höherer Geschwindigkeiten weniger dicht anzuordnen. Durch mehrere aufeinander folgende Messungen ist es möglich, die Masken adaptiv an die Strömung anzupassen und in jeder Region die maximale Datendichte zu erhalten. In den meisten Fällen ist die Berechnung eines einzelnen Vektorfeldes ausreichend, so dass die Serie der benötigten Aufnahmen sehr kurz gehalten werden kann. Die Verwendung eines dichteren Musters erlaubt dann die Berechnung eines dichteren Vektorfeldes. Die langen Bildserien, die nach dem Tagging-Prozess entstehen, ermöglichen Geschwindigkeitsauswertungen über einen längeren Zeitraum. Wenn ein sehr dichtes Muster verwendet wird, ist es aufgrund des Ineinanderlaufens kaum noch möglich, den zeitlichen Verlauf der Geschwindigkeiten zu betrachten. Bei der Dichte des Musters muss also ein Kompromiss 


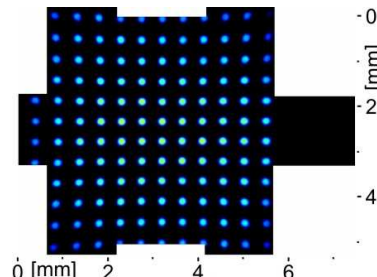

(a) $\stackrel{4}{=}=0 s$

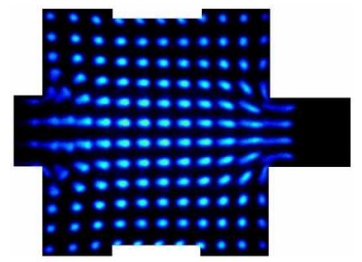

(d) $t=2,4 \mathrm{~s}$

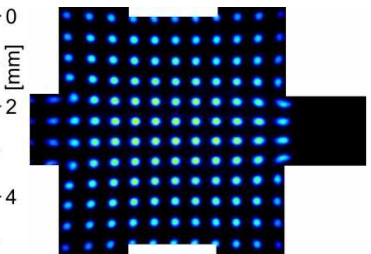

(b) $t=0,8 \mathrm{~s}$

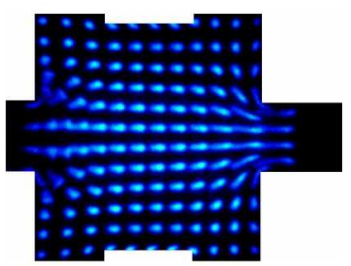

(e) $t=3,2 s$

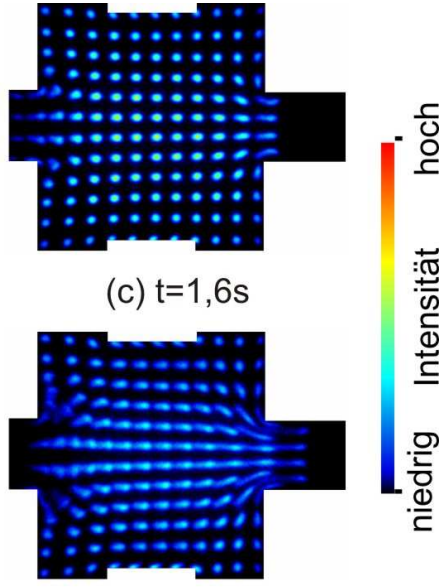

(f) $\mathrm{t}=4,0 \mathrm{~s}$

Abb. 3.14.: Bildsequenz einer MTV-Messung in der Mischkammer eines flachen Mikromischers, nur jedes achte Bild ist dargestellt

zwischen hoher Datendichte und langer Beobachtungszeit gefunden werden.

Mit diesen Erkenntnissen wurden erste Messungen in der Mischkammer durchgeführt. Verwendet wurde die große Mischkammer mit einer Kammergröße von $5 \times 5 \mathrm{~mm}$, wie in Abbildung 2.2 oben rechts gezeigt. Die Strömung verlief von links nach rechts durch die Mischkammer, wobei die Seitenkanäle ungenutzt blieben. Durch die Kammer wird also eine kurzzeitige Aufweitung des Strömungsquerschnitts bewirkt. Die zu erwartenden Strömungsgeschwindigkeiten sind im Zu- und Ablauf höher als innerhalb der Kammer. In Abbildung 3.14 sind Auszüge einer Bildserie gezeigt, die nach dem Schreibvorgang ausgelesen werden konnte. Ein Ergebnis der Geschwindigkeitsberechnung dieser Bildserie ist in Abbildung 3.15 gezeigt. Das Vektorfeld wurde aus acht einzelnen Feldern gemittelt, die aus Aufnahmen berechnet wurden, die nacheinander unter denselben Bedingungen aufgezeichnet wurden. Da ein relativ weites Puntkmuster verwendet wurde zeigen die Einzelbilder kein flächenfüllendes Vektorfeld, die Messung konnte dafür über einen längeren Zeitraum durchgeführt werden, ohne dass markierte Strömungsbereiche ineinander laufen. Da die zugrunde liegende Strömung laminar und stationär ist, konnten mehrere nacheinander gemessene Vektorfelder gemittelt werden. Durch diese Mittelung ergibt sich ein fast geschlossenes Vektorfeld, da durch die Wanderung der markierten Strömungsbereiche nach und nach an allen Stellen innerhalb der Kammer eine Geschwindigkeit gemessen werden konnte. Die Mittelung zeigt qualitativ genau die erwartete Strömung. Die Strömung weitet sich innerhalb der Kammer auf und die Geschwindigkeiten sinken im Vergleich zum schmaleren Zulauf. In Richtung des Ablaufs zeigt sich das inverse Verhalten. Die Strömung verläuft wieder in Richtung der Mitte, wo sich der Ablauf befindet, dabei steigen die Geschwindigkeiten. Da die Seitenkanäle verschlossen sind, sollten die Geschwindigkeiten im Zu- und Ablauf gleich hoch sein. Dieser Zustand ist im Vektorfeld korrekt wiedergegeben. 


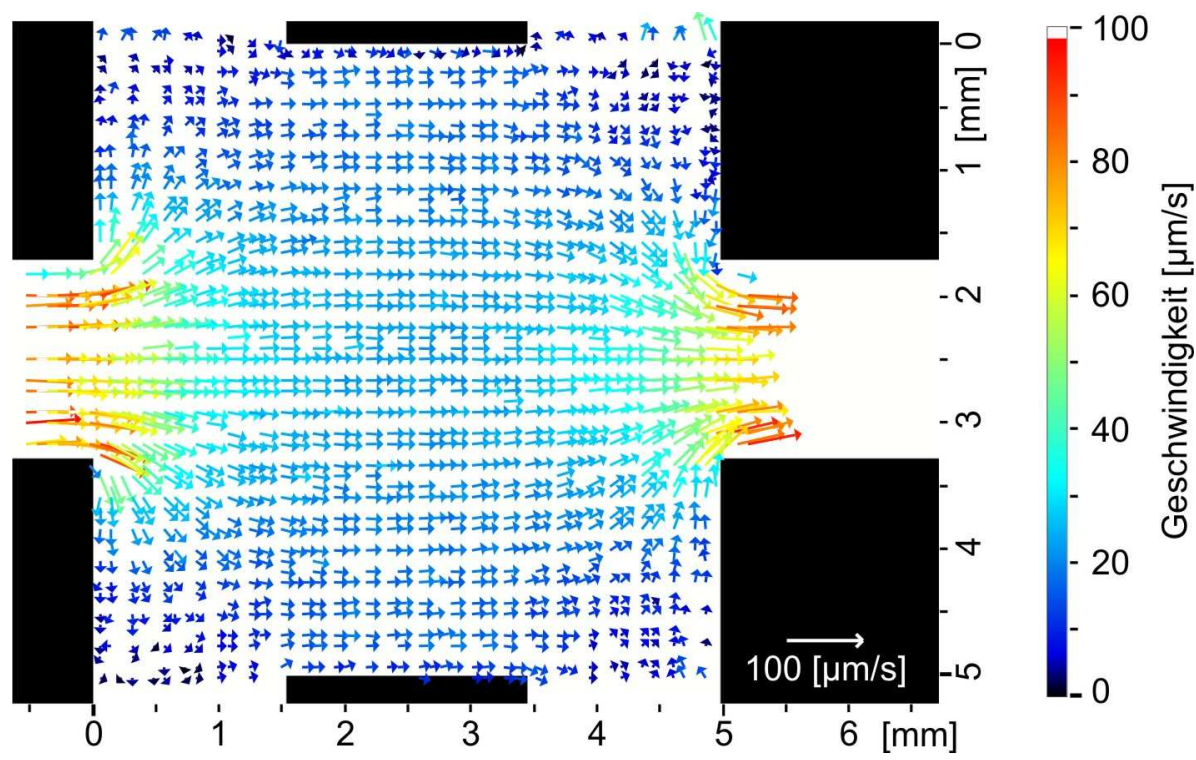

Abb. 3.15.: Vektorfeld einer MTV-Messung, berechnet aus der Bildsequenz aus Abbildung 3.14

\subsection{Quantitative Überprüfung des Verfahrens}

Nachdem die grundlegenden Voraussetzungen für eine Anwendung des Verfahrens bekannt sind, soll nun eine Abschätzung der quantitativen Genauigkeit der Ergebnisse durchgeführt werden. Dazu werden Messungen in einem einfachen Kanal durchgeführt und mit den Werten verglichen, die gleichzeitig mit dem Durchflussmesser aufgenommen wurden. Für einen Vergleich mit einem renommierten Verfahren wurden zusätzlich $\mu$ PIVMessungen durchgeführt. Aufgrund der komplexen, unterschiedlichen Aufbauten konnten die $\mu \mathrm{PIV}$-Messungen jedoch nicht simultan mit den MTV-Messungen durchgeführt werden. Als Vergleichsbasis dienen wiederum die mit dem Durchflussmesser bestimmten Strömungsgeschwindigkeiten. Sämtliche Messungen wurden an realistischen Mikrosystemen durchgeführt. Die Dimensionen wurden dabei im Vergleich mit den bisher genutzten Systemen deutlich verringert. Die Unterschiede wurden bereits in Abbildung 2.2 dargestellt. Bisher wurden die beiden oberen Geometrien eingesetzt. Die Breite des Kanals, bzw. die Ausdehnung der Kammer betrugen dort $5 \mathrm{~mm}$, um einfache Messungen zu ermöglichen. Im Folgenden wurden diese Maße auf $1 \mathrm{~mm}$ verkleinert um relevante mikrofluidische Dimensionen zu erreichen.

\subsubsection{D-MTV-Messungen}

Die geringeren Dimensionen der Fluidkanäle machten auch eine Verkleinerung der abgebildeten Muster notwendig. Die Abbildungsoptiken wurden zu diesem Zweck nicht verändert, die Verkleinerung geschah ausschließlich durch die Verwendung feinerer Mas- 
ken. Der sinnvoll nutzbare Maskenbereich verkleinerte sich um denselben Faktor fünf, um den auch die Kanalstrukturen verkleinert wurden. Um die Energie des Schreiblasers besser ausnutzen zu können, wurde dieser ebenfalls auf die kleinere Fläche gerichtet. Pro Schreibpuls erhöht sich auch die auftretende Leistungsdichte deutlich. Die Masken konnten damit nicht mehr wie bisher auf Folien gedruckt werden, da die Farbe ablatiert worden wäre. Die Masken wurden in diesem Fall aus Metall gefertigt. Genau wie die Metallfolien, die die Kanalgeometrien tragen, wurden die Masken durch ein Ätzverfahren hergestellt. Die hohen Leistungsdichten führten auch zu Problemen innerhalb der Mikrokanäle, wenn der Laserstrahl das Metall der Kanäle traf. In diesem Fall wurden die hohen Laserenergien stark absorbiert, so dass es in der Nähe der Kanalwände zu Blasenbildung aufgrund der starken Aufheizung der Metallwände kam. Der explosionsartige Verlauf der Blasenbildung sorgte für starke Verzerrungen der Muster. In den transparenten Bereichen, fern der Wände, konnten keine sichtbaren Einflüsse festgestellt werden. Probleme entstehen ausschließlich dort, wo die Energie des Lasers stark absorbiert wird, also an den metallenen Kanalwänden. Die Muster wurden daher beim Schreibvorgang durch eine Blende so weit eingegrenzt, dass das Laserlicht die Wände nicht erreichte.

Im ersten Schritt wurden Messungen bei verschiedenen Durchflussraten in einem geraden Kanal durchgeführt. Der Kanal ist im Querschnitt $1 \mathrm{~mm}$ breit und $200 \mu \mathrm{m}$ hoch (siehe Abbildung 2.2, unten links). Für die Messungen wurde der Aufbau verwendet, der in Abbildung 3.8 skizziert ist. Die Messungen wurden mit einer Reihe unterschiedlicher Masken bei mehreren Durchflussraten durchgeführt. Ein Beispiel der Ergebnisse dieser Messungen ist in Abbildung $3.16 \mathrm{zu}$ finden. Anhand der Verteilung der Vektoren ist die Geometrie der verwendeten Maske zu erkennen. Die Geschwindigkeiten können nur an den Stellen berechnet werden, an denen sich die Intensitätsgradienten der Punkte befinden. Die Räume dazwischen liefern keine sinnvollen Informationen und werden daher durch einen zusätzlichen Berechnungsschritt aussegmentiert. Das Vektorfeld ist sehr homogen, abgesehen von den Vektoren an den Rändern oben und unten. Hier liegen die Geschwindigkeiten aufgrund der Haftbedingungen an den Wänden niedriger, bzw. direkt an der Wand bei Null. Betrachtet wird der Bereich des Geschwindigkeitsplateaus in der Kanalmitte. Dadurch, dass die Taylordispersion in den Algorithmen des optischen Flusses bereits berücksichtigt wurde, erhält man die maximale Strömungsgeschwindigkeit, die sich bei dieser Kanalgeometrie in mittlerer Tiefe befindet. Durch eine gleichzeitige räumliche und zeitliche Mittelung im Bereich des Plateaus wurde eine Datenreduktion durchgeführt, so dass jedem Vektorfeld ein einzelner Wert (mittlerer Betrag der Geschwindigkeit) zugeordnet werden konnte. Neben der räumlichen Mittelung ist eine zeitliche Mittelung notwendig, da die berechneten Geschwindigkeiten im zeitlichen Verlauf schwanken. Diese Schwankungen werden vermutlich durch Laserschwankungen ausgelöst, die für eine ungleichmäßige Ausleuchtung sorgen. Diese Helligkeitsschwankungen resultieren in einem Fehler bei der Auswertung, da die Annahme eines konstanten Grauwertes verletzt wird (siehe Kapitel 3.1). Diese zufälligen Intensitätsschwankungen können in den Algorithmen nicht berücksichtigt werden, da sie keinem voraussehbaren Muster folgen. Bei der Auswertung werden sie als Geschwindigkeitsänderungen interpretiert. Die zeitliche Mittelung behebt dieses Problem, es werden dazu drei aufeinander 


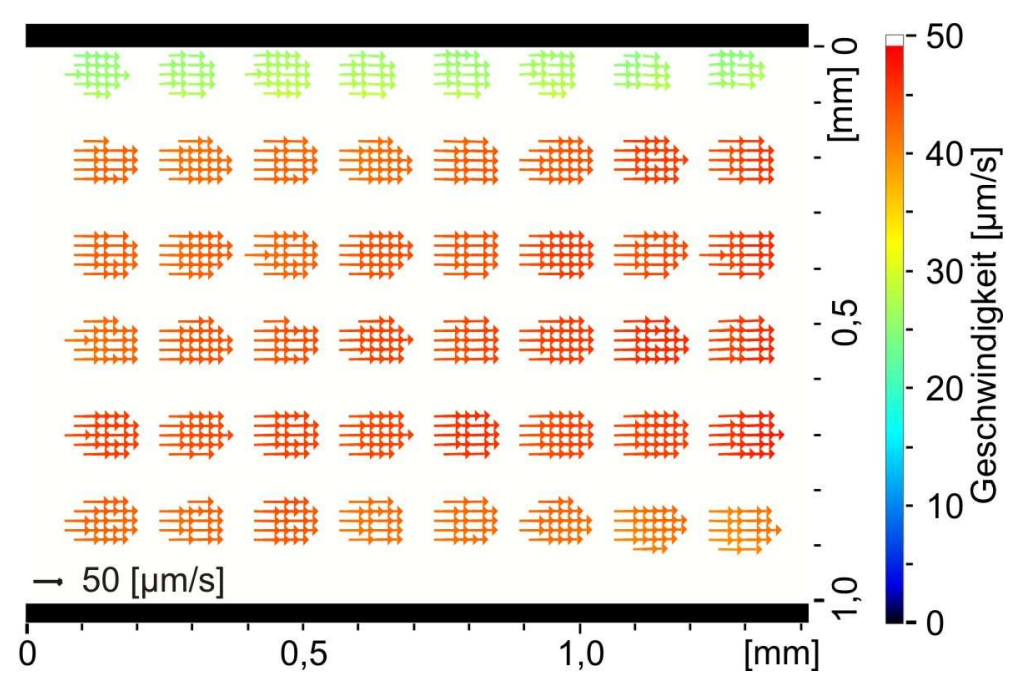

Abb. 3.16.: Vektorfeld einer MTV-Messung in einem geraden Mikrokanal

folgende Vektorfelder gemittelt.

Als Referenz zeichnet ein Durchflussmesser simultan den Volumenfluss auf. Dieser ist aufgrund der Inkompressibilität des Wassers bei einem vollkommen abgedichteten Kanal für jeden Kanalquerschnitt gleich. Da zusätzlich die Maße des Kanalquerschnitts im Messgebiet bekannt sind, kann aus den Durchflusswerten die mittlere Strömungsgeschwindigkeit berechnet werden. Für einen Vergleich ist es jedoch notwendig, dass die maximalen Strömungsgeschwindigkeiten im Bereich des Plateaus um die Kanalmitte bekannt sind. Für eine Abschätzung dieser Geschwindigkeiten werden bekannte Grenzfälle laminarer Strömungen verwendet. Die zwei wichtigsten Grenzfälle der Strömungsphysik sind die laminare Strömung im Rohr und die Strömung zwischen zwei unendlich ausgedehnten Platten. In beiden Fällen stellt sich ein parabelförmiges Geschwindigkeitsprofil ein. Im Fall der Platten findet sich das Profil in jedem Schnitt senkrecht zu den Platten, entlang der Strömung, im Rohr in jedem Querschnitt, senkrecht zum Rohrverlauf. Bei der Plattenströmung liegt die maximale Strömungsgeschwindigkeit genau um einen Faktor 1,5 höher als die mittlere Strömungsgeschwindigkeit, bei der Rohrströmung liegt sie doppelt so hoch. Der verwendete Kanal ist deutlich weiter als hoch und besitzt einen rechteckigen Querschnitt. Er entspricht somit eher der Plattenströmung. Durch die Begrenzungen entlang der Platten liegt die maximale Strömungsgeschwindigkeit in der Kanalmitte geringfügig höher als bei unendlich ausgedehnten Platten, da es auch in der zweiten Dimension eine Begrenzung gibt. In dieser Dimension bildet sich das schon bekannte Geschwindgikeitsplateau aus, während die Geschwindigkeit zu den Wänden hin gegen Null geht. Insgesamt liegt die maximale Strömungsgeschwindigkeit bei diesem Querschnitt also etwas höher als durch den Faktor 1,5 bei der Plattenströmung berücksichtigt wird. Der zusätzliche Faktor wurde experimentell bestimmt, indem bei einem gemessenen Geschwindigkeitsprofil die maximale Strömungsgeschwindigkeit durch 


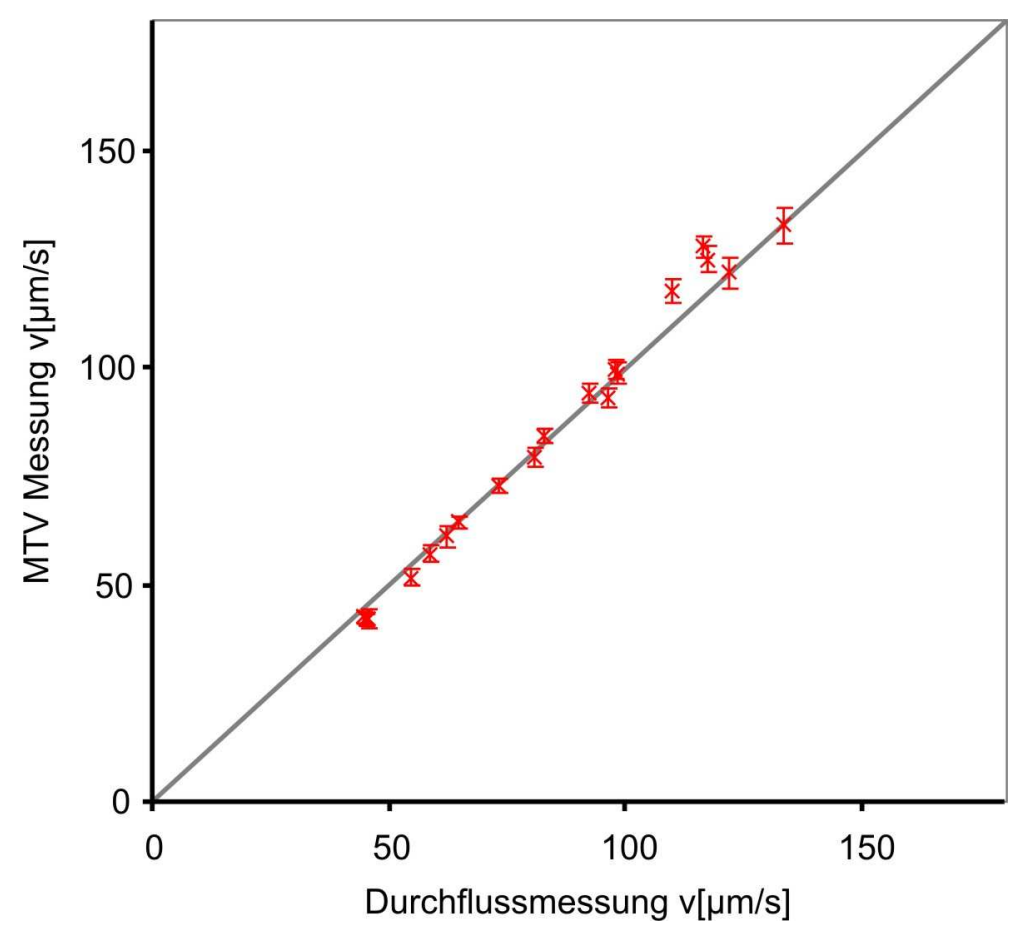

Abb. 3.17.: Vergleich der gemittelten Ergebnisse der MTV-Messungen mit den Referenzwerten des Durchflussmessers

die mittlere geteilt wird. Insgesamt ergibt sich, dass die maximale Strömungsgeschwindigkeit 1,77 mal höher liegt als die mittlere Strömungsgeschwindigkeit.

Die Strömungsgeschwindigkeiten, die aus den MTV-Messungen berechnet wurden, wurden gegen diejenigen aufgetragen, die durch die Durchflussmesung bestimmt werden konnten. Abbildung 3.17 zeigt diesen Vergleich. Bei idealer Übereinstimmung sollten die Werte auf der ebenfalls eingezeichneten Winkelhalbierenden liegen. Die Übereinstimmung der Werte ist sehr gut, die Abweichungen liegen im Bereich von $4 \%$. Das Verfahren ist also in der Lage quantitativ sehr genaue Aussagen zu machen. Dabei muss aber berücksichtigt werden, dass bei der Auswertung mehrfach Mittelungen durchgeführt wurden. Die verwendete Nachbarschaft zur Lösung der Geschwindigkeitsalgorithmen ist sehr groß eingestellt und sorgt für eine Art Mittelung. Dies erklärt auch, warum in Abbildung 3.16 am oberen Rand kein gleichmäßiges Absinken der Geschwindigkeiten zu erkennen ist. Die Vektoren im Bereich der obersten Reihe von Markierungen sind alle nahezu gleich lang, obwohl die Geschwindigkeit am Rand bei Null liegen sollte. Aufgrund der großen Nachbarschaften ist das Absinken nicht mehr zu erkennen. Außerdem war eine zusätzliche zeitliche Mittelung notwendig, um Fehler auszugleichen, die durch Intensitätsschwankungen bei der Fluoreszenzanregung auftreten. Die Auswertealgorithmen sind gegenüber solchen Schwankungen sehr anfällig, da sich die Fluoreszenzintensitäten, auf deren Basis die Geschwindigkeiten berechnet werden, ändern. 


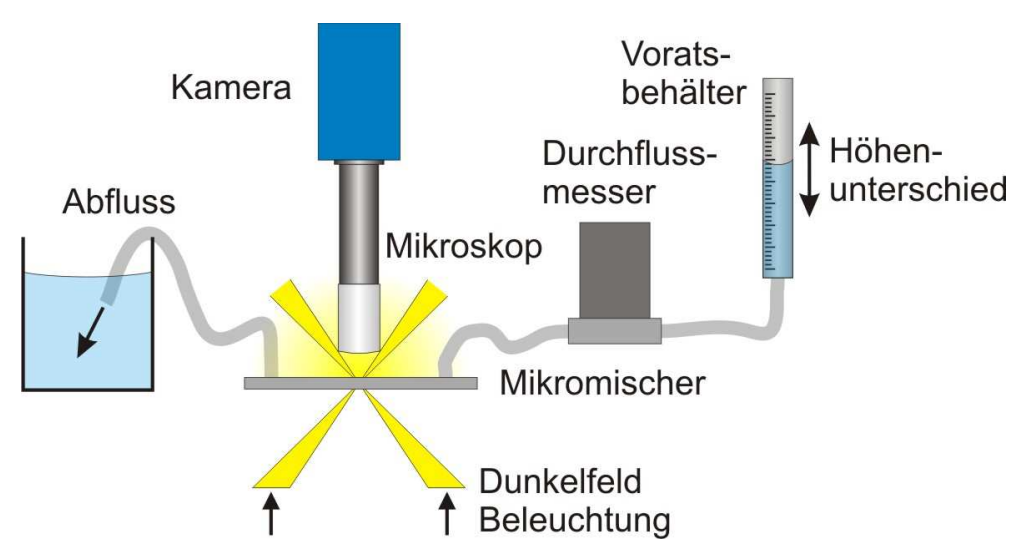

Abb. 3.18.: Experimenteller Aufbau für $\mu \mathrm{PIV}-$ Messungen

\subsubsection{Mikro-PIV-Messungen}

Für einen zusätzlichen Vergleich mit einem renommierten Verfahren wurden $\mu$ PIV-Messungen durchgeführt. Der verwendete experimentelle Aufbau ist in Abbildung 3.18 gezeigt. Die fluidischen Komponenten sind genauso aufgebaut wie bei den MTV-Messungen und wurden zwischen den Messungen nicht verändert. Es kommt also wiederum der gerade Kanal mit einer Breite von $1 \mathrm{~mm}$ und einer Höhe von $200 \mu \mathrm{m}$ zum Einsatz. Anstatt des caged dyes werden dem Wasser Partikel zugesetzt. Verwendet wurden Polystyrol Partikel mit einem Durchmesser von $1 \mu \mathrm{m}$, deren Dichte an die von Wasser angepasst ist (CAT\# 07310, Fa. Polyscience, Inc.). Durch die geringe Größe und die angepasste Dichte sollten die Partikel der Strömung sehr gut folgen. Für die Beobachtung wurde ein Mikroskop mit einer Dunkelfeldbeleuchtung verwendet. Diese Art der Beleuchtung verringert stark die Hintergrundstrahlung, da nur das, von den Partikeln gestreute, Licht die Beobachtungsoptiken erreichen kann. Für die Beleuchtung wird eine weiße StandardMikroskoplichtquelle eingesetzt. Zur Unterdrückung des Anregungslichtes bei der Detektion ist das Dunkelfeld-Beleuchtungsverfahren ausreichend.

Die Beobachtung findet durch ein 10x Mikroskopobjektiv (Fa. Zeiss) statt. Anhand eines Vorschlags von Meinhardt et al. [48] lässt sich die Korrelationstiefe eines Objektives berechnen. Dieser Wert gibt eine Vorstellung für den Tiefenbereich, in dem die Korrelationsalgorithmen die Partikel noch registrieren. Außerhalb dieser Schicht wird der Beitrag der unscharfen Partikel so gering, dass sie bei der Berechnung keine Rolle mehr spielen. Für das verwendete Objektiv liegt die Korrelationstiefe bei etwa $35 \mu \mathrm{m}$, damit kann der Scheitel der Geschwindigkeitsparabel ausreichend genau erfasst werden. Die Schärfeebene wird auf die Mitte des Kanals eingestellt, indem die Ränder der abschließenden Glasplatten oben und unten scharf eingestellt werden und aus deren Positionen die Kanalmitte bestimmt wird. Der Mikrokanal ist für diese Aufgabe durch Mikrometertriebe in allen Raumrichtungen präzise einstellbar montiert. Es wurden mehrere Geschwindigkeitsmessungen im selben Bereich wie bei den MTV-Messungen durchgeführt. Ein Beispiel der 


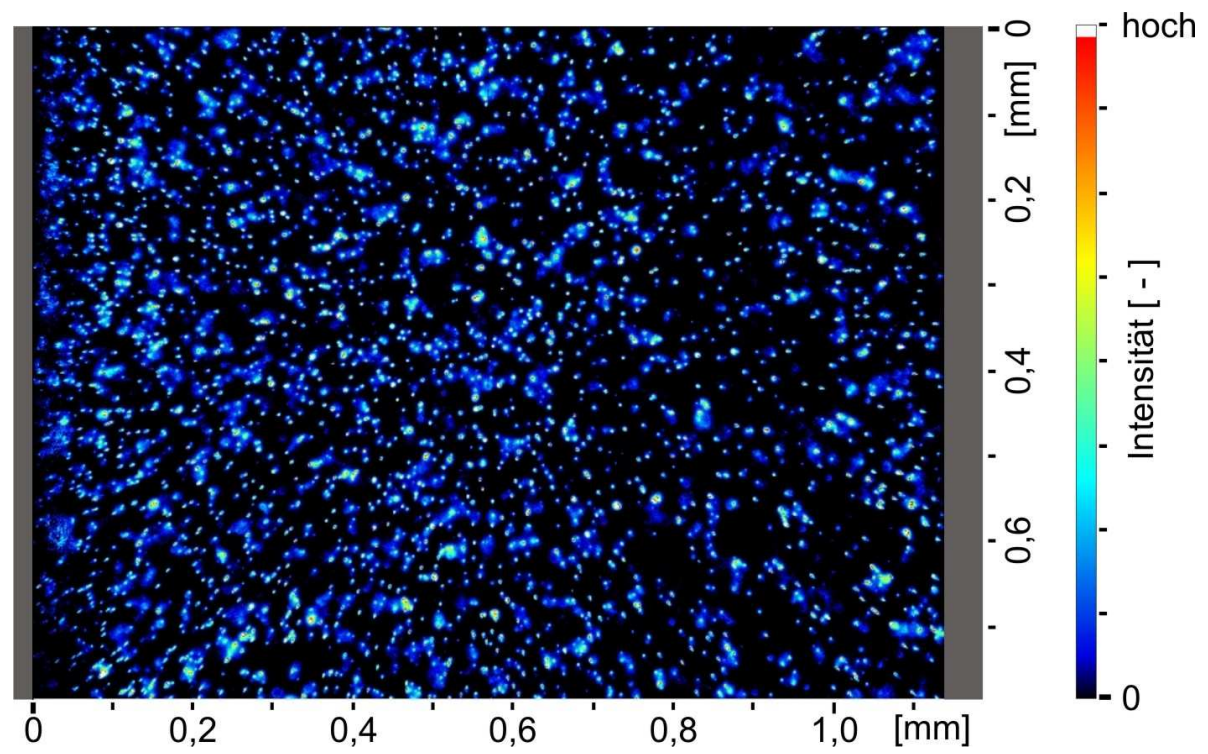

Abb. 3.19.: Partikelbild einer $\mu$ PIV-Messung nach Vorverarbeitung

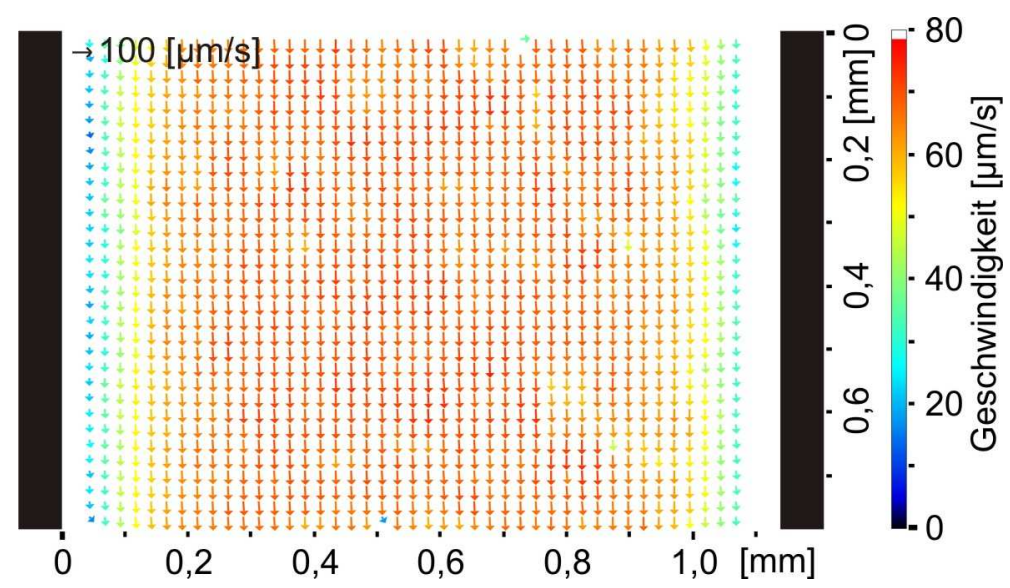

Abb. 3.20.: Vektorfeld einer $\mu \mathrm{PIV}$-Messung in einem geraden Mikrokanal

Partikelverteilung bei einer Messung ist in Abbildung $3.19 \mathrm{zu}$ sehen. Die Kanalbreite ist durch die grauen Balken links und rechts gekennzeichnet. Für die $\mu$ PIV-Messungen ist eine möglichst geringe Tiefenschärfe des verwendeten Objektivs notwendig. Damit einher geht ein kleineres Sichtfeld. Das verwendete Objektiv stellt einen guten Kompromiss aus Sichtfeldgröße und Tiefenschärfe dar. Die Kanalbreite wird zwar nicht vollständig erfasst, bei der späteren Datenreduktion werden aber sowieso nur noch die Maximalgeschwindigkeiten in der Kanalmitte ausgewertet. Die Partikelbilder wurden nach der Aufnahme korrigiert, um Hintergrundsignale und schwächeres Streulicht unscharfer Partikel zu entfernen. Zu diesem Zweck wurde aus einer Serie von 100 Aufnahmen für jeden 


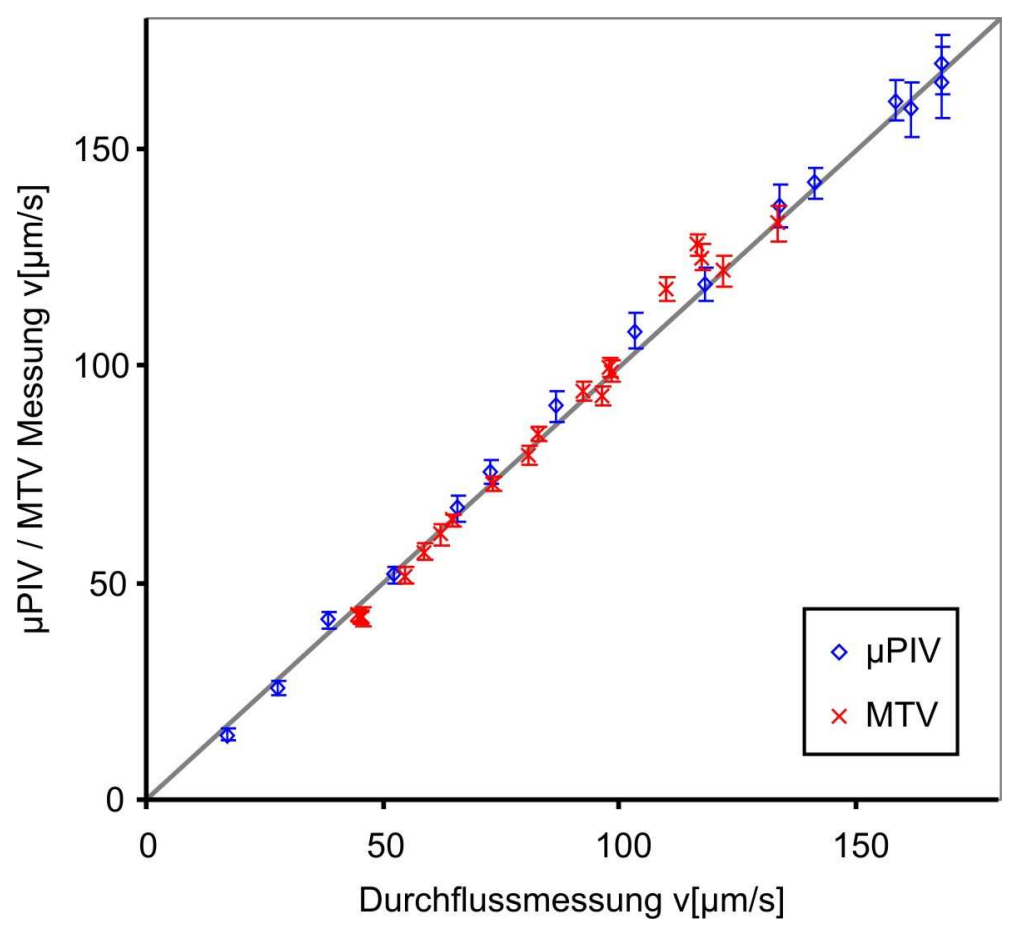

Abb. 3.21.: Vergleich der gemittelten Ergebnisse der MTV- und $\mu$ PIV-Messungen mit den Referenzwerten des Durchflussmessers

Bildpunkt der Minimumwert bestimmt und anschließend von allen Aufnahmen abgezogen. In einem zweiten Schritt wurde schwaches Streulicht durch Abzug eines festen Offsets entfernt. Damit werden auch unscharfe Partikel, die nicht innerhalb des Schärfebereichs liegen, aus den Aufnahmen entfernt. Beide Rechenschritte wurden bei dem in Abbildung 3.19 gezeigten Bild bereits durchgeführt. Nach dieser Vorbearbeitung wurden die Geschwindigkeiten aus Paaren von jeweils zwei aufeinander folgenden Partikelbildern durch einen Kreuzkorrelationsalgorithmus bestimmt. Für die Berechnungen wurde eine kommerziell erhältliche PIV-Software verwendet (DaVis, Fa. LaVision). Ein Beispiel für die resultierenden Vektorfelder ist in Abbildung $3.20 \mathrm{zu}$ sehen. Deutlich zu erkennen ist das ausgebildete laminare Geschwindigkeitsprofil. In der Mitte entsteht ein Plateau gleich hoher Geschwindigkeiten, während sie zu den Kanalwänden links und rechts abfällt.

Auch für diese Daten wurde ein Vergleich mit den Werten des Durchflussmessers durchgeführt. In Abbildung 3.21 sind die Ergebnisse auch mit den Ergebnissen der MTV-Messungen verglichen. Sowohl die Ergebnisse der $\mu$ PIV-Messungen, als auch die Ergebnisse der MTV-Messung wurden gegen die jeweils simultan gemessenen Werte des Durchflussmessers aufgetragen. Es zeigen sich sehr gute Übereinstimmungen. Die $\mu$ PIV-Messungen bestätigen also die guten Ergebnisse der MTV-Messungen. 

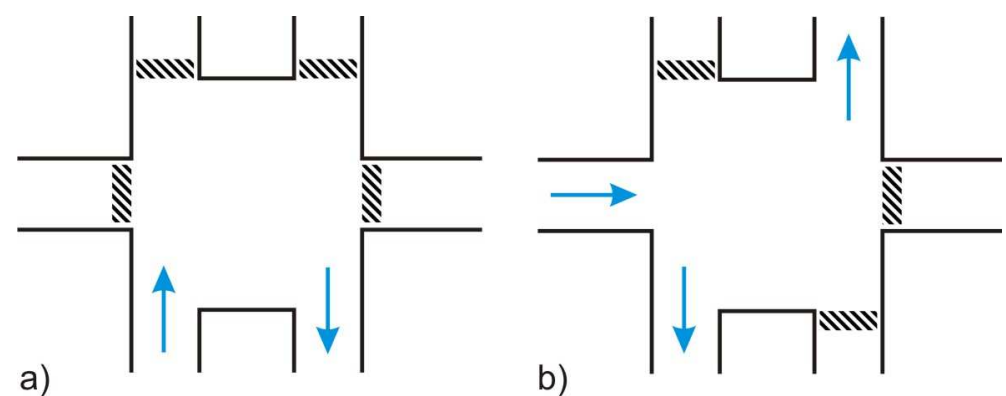

Abb. 3.22.: Strömungskonfigurationen in der Mikromischkammer

\subsection{Messungen in der Mischkammer}

Messungen unter realitätsnahen Bedingungen wurden in der Mikromischkammer durchgeführt. Die Kammergröße wurde dazu auf $1 \times 1 \mathrm{~mm}$ verkleinert. Die Kanäle sind nach der Verkleinerung noch $300 \mu \mathrm{m}$ breit. Die Tiefe der Kanäle wurde nicht verändert und liegt weiterhin bei $200 \mu \mathrm{m}$. Das Schreibmuster wurde ebenfalls verkleinert, wie im vorigen Abschnitt beschrieben. Das Muster wurde dabei nur innerhalb der Mischkammer in die Flüssigkeit geschrieben, so dass der UV-Puls die Wände nicht trifft. Die Anschlüsse am Mikromischer wurden unterschiedlich verwendet, um mehrere einfache Strömungen in der Mischkammer zu erzeugen. Abbildung 3.22 zeigt die beiden Varianten, die im Folgenden präsentiert werden sollen. Die blauen Pfeile zeigen die Flussrichtungen der jeweils offenen Anschlüsse, die schraffierten Balken kennzeichnen verschlossene Anschlüsse. In Teilbild a wurde die Kammer von links unten nach rechts unten durchströmt, die restlichen Anschlüsse wurden blockiert. Teilbild b zeigt eine zweite Strömung, bei der die Strömung von links in die Kammer geleitet wird. In diesem Fall wurden zwei Abflusskanäle geöffnet, nämlich die Anschlüsse unten links und oben rechts. Die restlichen Kanäle bleiben verschlossen.

Ein Ergebnis der Geschwindigkeitsmessung der Konfiguration aus Abbildung 3.22a ist in Abbildung 3.23 gezeigt. Der Durchflussmesser registrierte einen Volumenstrom von $0,8 \mu \mathrm{l} / \mathrm{min}$, dies entspricht, bezogen auf die Querschnittsmaße eines Kanals, einer mittleren Strömungsgeschwindigkeit von $222 \mu \mathrm{m} / \mathrm{s}$. Das verwendete Punktmuster besteht aus regelmäßig angeordneten Punkten mit einem Durchmesser von etwa $80 \mu \mathrm{m}$ und einer Periodizität von $120 \mu \mathrm{m}$. Die Strömung verläuft, wie zu erwarten, von links unten nach rechts unten in der Kammer. Die Geschwindigkeiten sind am Zulauf und Abfluss am höchsten und nehmen nach oben, also in die Breite der Kammer, nach und nach ab. Die Strömungsrichtungen nahezu aller Vektoren geben schlüssig eine rechtsgerichtete, halbkreisförmige Bewegung durch die Mischkammer wieder.

Abbildung 3.24 zeigt ein Ergebnis der zweiten Strömungskonfiguration, wie sie in Abbildung $3.22 \mathrm{~b}$ gezeigt wurde. Es wurde vom Durchflussmesser ein Volumenfluss von $1,0 \mu \mathrm{l} / \mathrm{min}$ registriert. Die mittlere Strömungsgeschwindigkeit bezogen auf den Querschnitt eines Kanals beträgt damit $278 \mu \mathrm{m} / \mathrm{s}$. Das verwendete Punktmuster ist dasselbe 


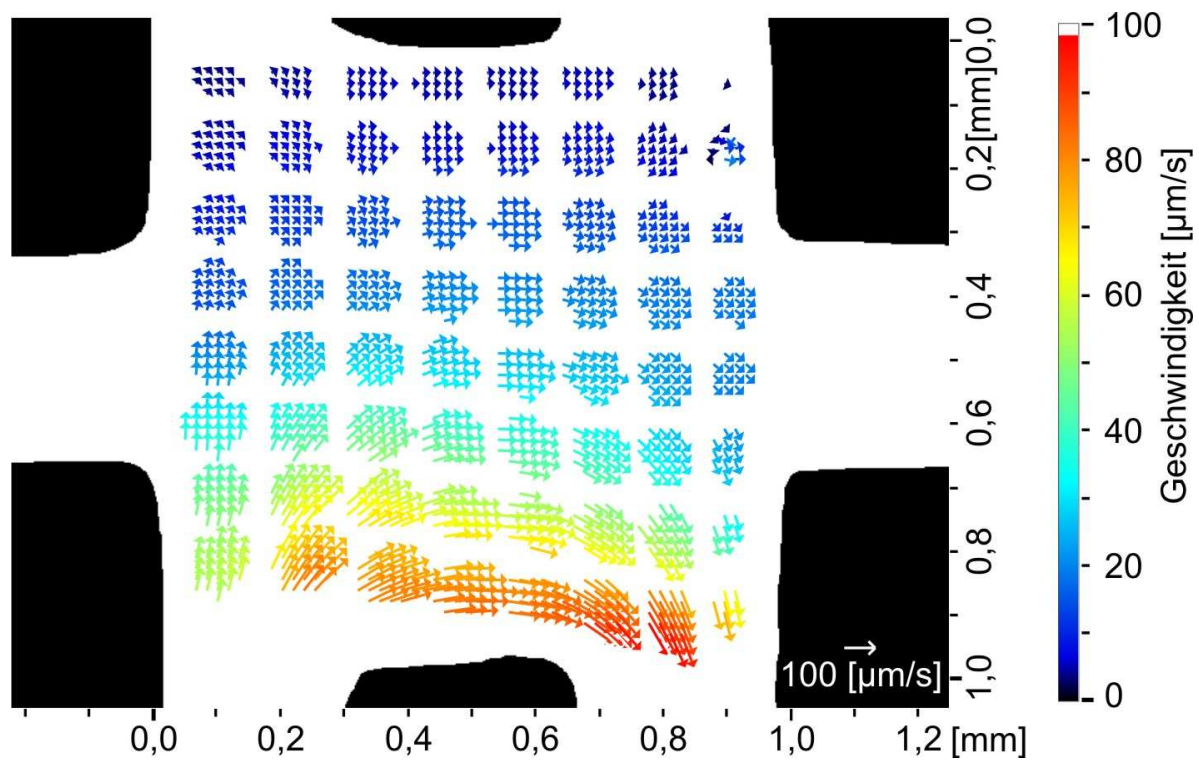

Abb. 3.23.: Vektorfeld einer MTV-Messung in der Mischkammer eines Mikromischers bei Strömungskonfiguration a aus Abbildung 3.22

wie bei der ersten vorgestellten Messung. Auch in diesem Fall entspricht der Verlauf der Vektoren der erwarteten Strömung. Vom Zulauf links verlaufen zwei Strömungen zu den beiden Auslässen unten links und oben rechts. Der Zustrom teilt sich schon kurz nach Eintritt in die Kammer in die zwei Teilströme auf. Der Einflussbereich der nach unten gerichteten Strömung ist dabei eher klein. Die Vektoren zeigen auch unten rechts in der Kammer eine Strömung, die sich wieder nach oben zum zweiten Auslass richtet.

\subsection{Fazit}

Durch die Kombination von zweidimensionaler Molecular Tagging Velocimetry mit einer Geschwindigkeitsauswertung auf Basis der Methode des optischen Flusses konnte ein zweidimensionales Geschwindigkeitsmessverfahren für mikrofluidische Systeme realisiert werden. Für den Taggingvorgang wurde eine sehr variable Mustererzeugung durch eine Maskenabbildung genutzt, die eine dauerhafte Fixierung eines Musters in einem so genannten caged dye erlaubt. Für die Auswertung der Tagging-Bildsequenzen wurde anstelle der weit verbreiteten Korrelationsalgorithmen eine alternative Technik auf Basis der Methode des optischen Flusses genutzt. Dadurch war es möglich, die Algorithmen derart zu modifizieren, dass sowohl die Diffusion des Farbstoffes als auch die Taylordispersion erfasst und in die Auswertung einbezogen werden konnten.

Bei den Messungen zur Überprüfung der quantitativen Genauigkeit des Verfahrens ergeben sich sehr gute Übereinstimmungen mit den Referenzwerten. Da diese Übereinstimmung auch bei einer Kontrollmessung mit dem renommierten Verfahren der $\mu$ PIV 


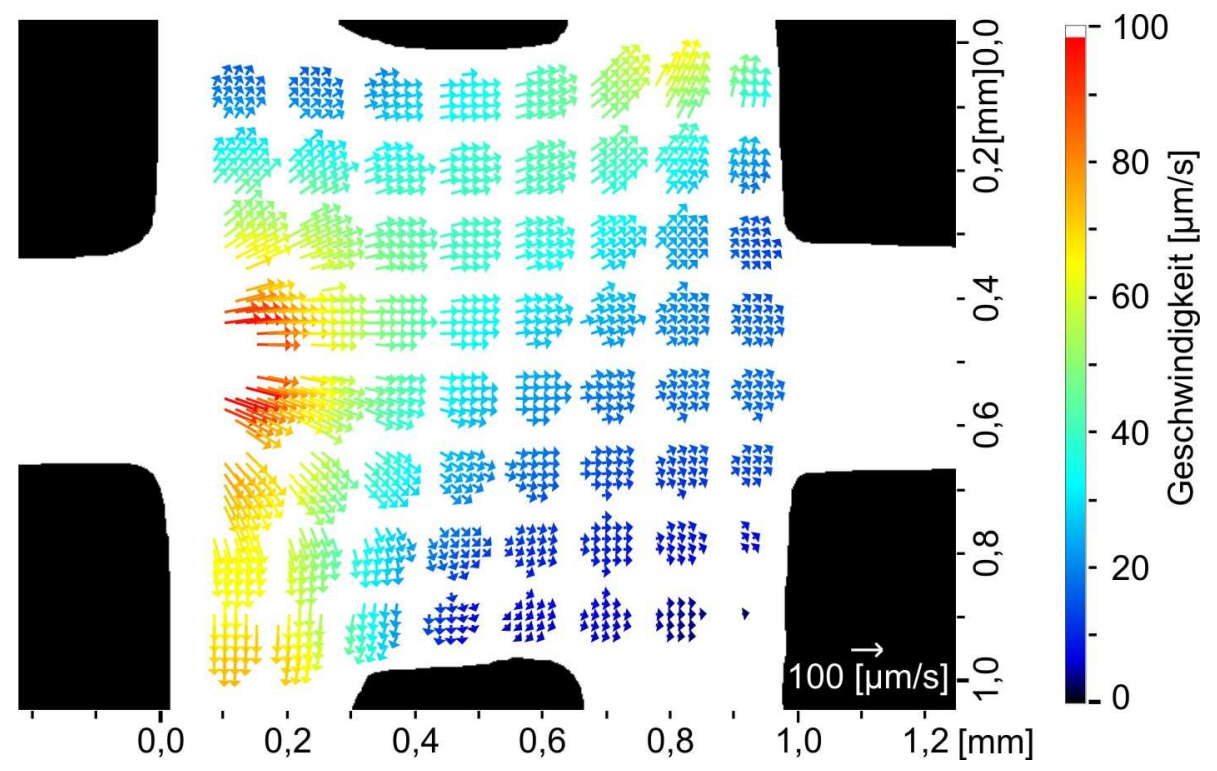

Abb. 3.24.: Vektorfeld einer MTV-Messung in der Mischkammer eines Mikromischers bei Strömungskonfiguration b aus Abbildung 3.22

gefunden werden konnte, ist davon auszugehen, dass bei den Messungen keine systematischen Fehler auftraten. Die hohen Genauigkeiten (mittlerer Fehler $4 \%$ ) konnten jedoch nur bei starker räumlicher und zeitlicher Mittelung der Vektorfelder realisiert werden. Prinzipiell ist der verwendete Auswertealgorithmus sehr anfällig gegenüber nicht modellierten Grauwertänderungen. Verschiedene Quellen von Intensitätsschwankungen, die diese Grauwertänderungen auslösen, konnten identifiziert werden. Der Farbstoff hat nach dem Schreibvorgang eine relativ lange Zerfallszeit, wodurch zumindest das erste aufgenommene Bild sich in seiner Intensität deutlich von den folgenden unterscheiden kann. Problematisch ist weiterhin ein Ineinanderlaufen der markierten Strömungsbereiche. Wie gezeigt werden konnte, können diese Effekte bei unzweckmäßiger Taggingstruktur zu großen Fehlern führen. Die dritte bekannte Quelle sind Intensitätsschwankungen des verwendeten Anregungslasers. Die ersten beiden Fehlerquellen können durch eine geeignete Nutzung der Mustersequenzen, bzw. ein passendes Markierungsmuster einfach umgangen werden. Die Intensitätsschwankungen des Anregungslasers sind ein grundsätzliches Problem des verwendeten Aufbaus und sollten für präzise Messungen in Zukunft von vorn herein ausgeschlossen werden. Grundsätzlich wären aber auch robustere Algorithmen wünschenswert. In diesem Punkt ist die Messtechnik den äußerst robusten konventionellen Korrelationsalgorithmen derzeit noch deutlich unterlegen.

Den abschließenden Entwicklungsstand des Messverfahrens demonstrierten mehrere Messungen in einer Mikromischkammer. Die Maskenabbildung wurde an die sehr kleinen mikrofluidischen Dimensionen angepasst, so dass ein scharf aufgelöstes Punktmuster erzeugt werden konnte. Die kleinsten genutzten Punktdurchmesser lagen bei etwa $50 \mu \mathrm{m}$. Die Begrenzung für die Punktgröße war in diesem Fall allein durch die vorhandenen Mas- 
ken gegeben. Mit einer entsprechend präzisen Abbildung würden allerdings noch deutlich kleinere Punktmuster erzeugt werden können. Die Auswertung mit den angepassten Algorithmen auf Basis der Methode des optischen Flusses lieferte sinnvolle Vektorfelder, die den erwarteten Strömungsverlauf sehr gut wiedergaben. 


\section{Der Raman-Effekt}

Einige der wichtigsten Methoden der Spektroskopie basieren auf dem Raman-Effekt. Die Stärke dieser Methoden liegt in der Möglichkeit, verschiedenste chemische Verbindungen zu identifizieren bzw. ihre Struktur aufzuklären. Der Raman-Effekt ist ein inelastischer Streuprozess, bei dem ein Photon an einem Molekül gestreut wird und dabei ein Energieaustausch zwischen Photon und Molekül stattfindet. Auf das Molekül wird bei dem Streuprozess eine Energie übertragen, durch die ein Schwingungs- oder Rotationszustand des Moleküls angeregt wird. Die Wellenlänge des gestreuten Photons ist gegenüber der des einfallenden also in den roten Spektralbereich verschoben. Auch der umgekehrte Prozess ist möglich. Wenn ein Photon auf ein Molekül trifft, das sich bereits im angeregten Zustand befindet, dann geht dieses in den Grundzustand über und das gestreute Photon besitzt eine entsprechend größere Energie, seine Wellenlänge ist also ins blaue verschoben. Wenn das Molekül mit monochromatischem Licht bestrahlt wird, ergeben sich auf diese Weise spektrale Verschiebungen des eingestrahlten Lichts, die charakteristisch für die streuende Molekülspezies sind. Dieser spektrale „Fingerabdruck“ liefert demnach wertvolle Hinweise auf den Aufbau des Moleküls und seine innere Struktur.

Die Eigenschaften der Ramanstreuung sind sehr gut ersichtlich aus Energiediagrammen des Prozesses. Abbildung 4.1 zeigt die Vorgänge für Rayleigh- und Ramanstreuung. Quantenmechanisch gelten die folgenden Auswahlregeln für die Übergänge zwischen den Energieniveaus des Moleküls:

$$
\begin{aligned}
& \Delta \nu=0 \quad \Delta J= \pm 2 \quad \text { für die Rotationsübergänge } \\
& \Delta \nu= \pm 1 \quad \Delta J=0, \pm 2 \text { für die Vibrationsübergänge }
\end{aligned}
$$

Ein Molekül wird nach Absorption eines Photons in einen virtuellen angeregten Zustand versetzt. Dieser Zustand entspricht einer Verzerrung der Elektronenverteilung einer kovalenten Bindung des Moleküls. Das Molekül relaxiert nahezu instantan in ein niedrigeres Energieniveau, indem ein Photon emittiert wird. Dabei kann es zurück auf das ursprüngliche Niveau fallen, wie in Abbildung 4.1a der Fall, dieser Vorgang wird Rayleighstreuung genannt. Die gestreuten Photonen besitzen dieselbe Wellenlänge wie die zuvor eingestrahlten. Es kann auch vorkommen, dass das Molekül in einen höheren Schwingungs- bzw. Rotationszustand übergeht. Das abgestrahlte Photon besitzt dann eine niedrigere Energie als das zuvor eingestrahlte, wie in Abbildung 4.1b. Dieser Vorgang wird als Stokes-Ramanstreuung bezeichnet und führt zu einer Rotverschiebung des eingestrahlten Lichts. Da bei einer definierten Temperatur nach Boltzmann immer einige Moleküle in thermisch angeregten Zuständen existieren, kann auch der umgekehrte Fall eintreten, wie in Abbildung 4.1c. Das emittierte Photon hat dann eine höhere Energie als zuvor und das Molekül geht in einen niedrigeren Energiezustand über. In diesem Fall 

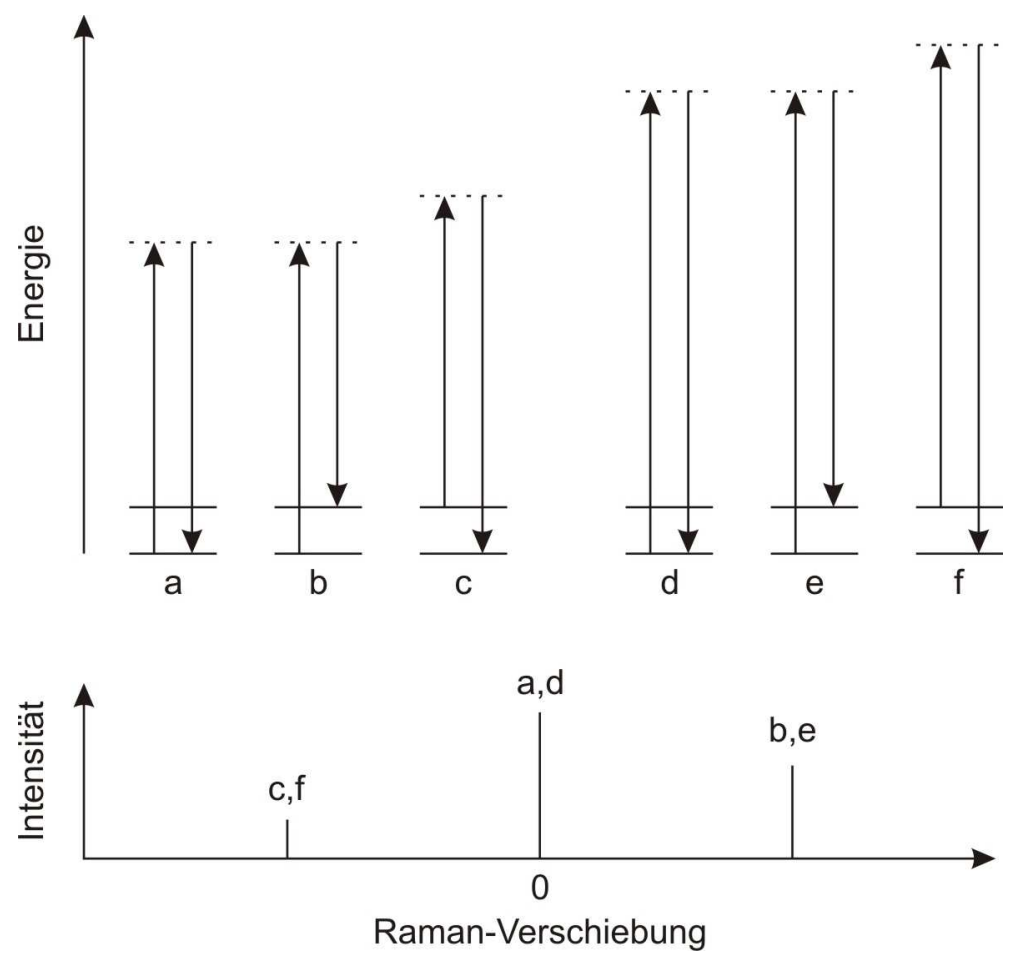

Abb. 4.1.: Energiediagramme zur Ramanstreuung

spricht man auch von Antistokes-Ramanstreuung. Das bei diesem Streuprozess emittierte Licht ist gegenüber dem eingestrahlten blau verschoben. Die Energieschemata in Abbildung 4.1d-f zeigen dieselben Übergänge bei Anregung mit Photonen einer höheren Energie. Das virtuelle Energieniveau liegt dann entsprechend höher. Die Energiedifferenz zwischen eingestrahltem Licht und emittiertem Licht ist jedoch auch hier gleich der Energiedifferenz der beteiligten Molekülzustände, sodass sich die gleiche Energieverschiebung der eingestrahlten Photonen ergibt. Die resultierenden Linien im Spektrum sind in der unteren Ansicht in Abbildung $4.1 \mathrm{zu}$ finden. Sie unterscheiden sich nicht von denen, die sich aus den Fällen a bis c ergeben, da die Anregungswellenlänge keinen Einfluss auf die Form des Ramanspektrums hat. Der Rayleigh-gestreute Anteil des Lichts (Abbildung 4.1a und d) ist nicht spektral verschoben zur Anregungswellenänge und liegt daher bei einer Ramanverschiebung von Null. Die Stokeslinie ergibt sich aus den Übergängen b und e und die Antistokeslinie aus c und f. Die Stokeslinie ist dabei wesentlich schwächer als die Rayleighlinie, die Antistokeslinie ist noch einmal deutlich schwächer. Dies liegt an den erheblich geringeren Streuquerschnitten der beiden Ramanprozesse. Die Wahrscheinlichkeit, dass ein Photon Raman-verschoben gestreut wird, ist um mehrere Größenordnungen kleiner, als dass es unverschoben gestreut wird. Die Wahrscheinlichkeit für das Auftreten von Antistokes Ramanlicht ist aufgrund der Boltzmannverteilung wiederum deutlich kleiner. 
Die Raman-Verschiebung ergibt sich also ausschließlich aus den Energiezuständen des Moleküls. Daher ist es sinnvoll, die Verschiebung als Energieäquivalent anzugeben. Es hat sich historisch ergeben, dass in diesem Zusammenhang Wellenzahlen verwendet werden, die üblicherweise in $\mathrm{cm}^{-1}$ notiert werden. Der lineare Zusammenhang zwischen Energie und Wellenzahl ist gegeben aufgrund der folgenden Gleichungen:

$$
\begin{aligned}
E=h \nu= & \frac{h c}{\lambda}=h c k \\
\text { mit } h \quad & : \text { Plancksche Konstante } \\
\nu & : \text { Frequenz des Lichts } \\
c & : \text { Lichtgeschwindigkeit } \\
\lambda & : \text { Wellenlänge } \\
k=\frac{1}{\lambda} & : \text { Wellenzahl }
\end{aligned}
$$

Eine Wechselwirkung des anregenden Lichts kann sowohl mit den Molekülen eines gasförmigen oder flüssigen Stoffes, als auch mit den Gitterstrukturen eines Festkörpers auftreten. Klassisch betrachtet induziert eine einfallende Lichtwelle mit der elektrischen Feldstärke $\vec{E}$ ein elektrisches Dipolmoment $\vec{p}_{i n d}$, welches ein bereits vorhandenes permanentes Dipolmoment $\overrightarrow{p_{0}}$ überlagert.

$$
\vec{p}=\vec{p}_{0}+\vec{p}_{\text {ind }}=\vec{p}_{0}+\alpha \cdot \vec{E}
$$

Dabei ist $\alpha$ die Polarisierbarkeit des angeregten Moleküls, sie wird mathematisch als Tensor zweiter Stufe formuliert. Die Polarisierbarkeit und das Dipolmoment hängen funktional nur von den Koordinaten der Elektronen und Kerne ab, so dass es geschickt ist, zu einer Darstellung in Normalkoordinaten überzugehen. Für kleine Verschiebungen der Kerne und Elektronen gegeneinander können $\vec{p}$ und $\alpha$ durch eine Taylorentwicklung um den Nullpunkt der Verschiebung entwickelt werden. Wenn man beide Entwicklungen nach dem linearen Term abbricht, ergeben sich die beiden folgenden Gleichungen für Polarisierbarkeit und Dipolmoment:

$$
\begin{gathered}
\alpha=\alpha_{0}+\sum_{n}\left(\frac{\partial \alpha}{\partial Q_{n}}\right)_{0} Q_{n} \\
\vec{p}=\vec{p}_{0}+\sum_{n}\left(\frac{\partial \vec{p}}{\partial Q_{n}}\right)_{0} Q_{n}
\end{gathered}
$$

Die Summen erstrecken sich über alle auftretenden Schwingungen. Die Zahl der Summationen hängt von der Gestalt des Moleküls und damit seinen Freiheitsgraden ab. Von allen möglichen Freiheitsgraden werden diejenigen der Translation abgezogen, so dass nur die Schwingungs- und Rotationsfreiheitsgrade bleiben. Für ein einfaches Molekül, das aus $N$ Atomen besteht, wären dies zum Beispiel $3 N-3$ Freiheitsgrade. Eine mögliche Darstellung der Normalkoordinaten sind harmonische Schwingungen mit den Amplituden $Q_{n, 0}$ und den Frequenzen $\omega_{n}$ :

$$
Q_{n}(t)=Q_{n, 0} \cdot \cos \omega_{n} t
$$


Auch das elektrische Feld des anregenden Lichts kann als harmonische Schwingung mit der Frequenz $\omega$ und Amplitude $\vec{E}_{0}$ formuliert werden

$$
\vec{E}(t)=\vec{E}_{0} \cdot \cos \omega t
$$

so dass sich insgesamt das zeitabhängige elektrische Dipolmoment im Molekül folgendermaßen zusammensetzt:

$$
\begin{aligned}
\vec{p}(t)=\vec{p}_{0} & +\sum_{n}\left(\frac{\partial \vec{p}}{\partial Q_{n}}\right)_{0} \cdot \cos \omega_{n} t \\
& +\alpha_{0} \vec{E}_{0} \cdot \cos \omega t \\
& +\left(\sum_{n}\left(\frac{\partial \alpha}{\partial Q_{n}}\right)_{0} Q_{n, 0} \cdot \cos \left(\omega \pm \omega_{n}\right) t\right) \cdot \frac{\vec{E}_{0}}{2}
\end{aligned}
$$

Die beiden ersten Terme beschreiben das permanente Dipolmoment und den Anteil, der mit den Schwingungen der Moleküle oszilliert. Die Energiebeträge dieser Schwingungen werden bei Wechselwirkung der Moleküle mit infraroter Strahlung erreicht. Der dritte Term entspricht der Rayleigh-Streuung. Die Frequenz der abgegebenen Streustrahlung bleibt dabei gleich derjenigen des eingestrahlten Lichts. Die Amplitude ist einerseits direkt proportional zur Amplitude des eingestrahlten Lichts und andererseits zur Polarisierbarkeit in Richtung des elektrischen Feldes. Der vierte Term in Gleichung 4.7 beschreibt die Auswirkungen des induzierten Dipolmoments auf die Frequenzen der gegeneinander schwingenden Kerne $\omega_{n}$. Der emittierte Strahlungsanteil ist die inelastische Ramanstreuung, die für rot- und blauverschobene Spektrallinien sorgt. Historisch werden die Spektrallinien für die beiden Strahlungsanteile als Stokes-Linien für die Frequenzen $\omega-\omega_{n}$ und Antistokes-Linien für $\omega+\omega_{n}$ bezeichnet. Es ist ersichtlich, dass für das Auftreten der Antistokes-Linien bereits eine thermische Besetzung des Energiezustands bestanden haben muss, da dem einfallenden Licht Energie zugeführt wird. Voraussetzung für das Auftreten von Raman-Streustrahlung ist, dass

$$
\left(\frac{\partial \alpha}{\partial Q_{n}}\right)_{0} \neq 0
$$

gilt. In diesem Fall wird die zugehörige Molekülschwingung als Raman-aktiv bezeichnet.

Das angeregte Molekül verhält sich aufgrund des Dipolmoments, das sich periodisch mit der Zeit ändert, wie ein Hertzscher Dipol. Die emittierte Strahlungsintensität pro Raumwinkel $\Omega$ ergibt sich daher aus

$$
I^{\Omega}(\Theta)=\frac{|\vec{p}|^{2} \omega^{4} \sin ^{2} \Theta}{32 \pi^{2} \epsilon_{0} c^{3}} .
$$

Hier ist $\Theta$ der Winkel zwischen Detektionsrichtung und Richtung des induzierten Dipolmoments, die durch den Polarisierbarkeitstensor $\alpha$ vorgegeben wird. Eine Beobachtung parallel zur Polarisationsrichtung würde darin resultieren, dass kein gestreutes Ramanlicht detektiert würde. Die maximalen Intensitäten ergeben sich bei einer Detektion unter einem Winkel von $90^{\circ}$ zur Ausrichtung des Dipolmoments. 


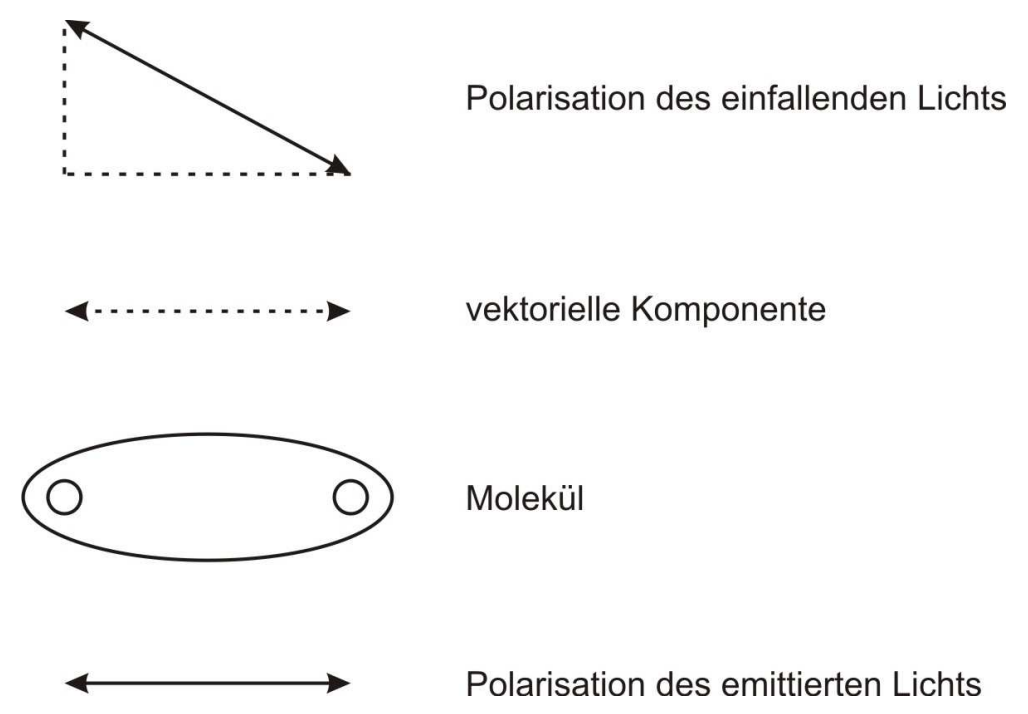

Abb. 4.2.: Einfluss der Polarisationsrichtung des Anregungslichts auf die Intensität des emittierten Ramanstreulichts

Auch die Ausrichtung der Polarisation des Anregungslichts spielt eine Rolle, wenn es um die Intensitäten des Ramanstreulichts geht. Diesen Zusammenhang verdeutlicht Abbildung 4.2. Die Polarisation des einfallenden Lichts unterscheidet sich von der einzig möglichen Schwingungsrichtung des zweiatomigen Moleküls, das angeregt werden soll. Entsprechend der Ausrichtung des Polarisierbarkeitstensors für die Schwingung dieses Moleküls wird nur die passende vektorielle Komponente der Polarisation des einfallenden Lichts absorbiert. Die Polarisation des emittierten Lichts liegt ebenfalls in dieser Richtung, sie unterscheidet sich also ebenfalls von der des einfallenden Lichts.

Die detektierte Intensität des Ramanstreulichts einer spezifischen Linie des diskreten Ramenspektrums eines Moleküls kann aus der folgenden Gleichung bestimmt werden:

$$
\begin{array}{cl}
I_{i}=c I_{0} \Omega V_{D} & N_{i}\left(\frac{\partial \sigma}{\partial \Omega}\right)_{i} \\
\text { mit } c & : \text { Konstante des Detektionssystems } \\
I_{0} & : \text { Intensität des Anregungslichts } \\
N_{i} & : \text { Anzahl der Moleküle der Spezies i } \\
\Omega & : \text { Raumwinkel der Detektion } \\
V_{D} & : \text { Detektionsvolumen } \\
\left(\frac{\partial \sigma}{\partial \Omega}\right)_{i} & : \text { Raman-Streuquerschnitt der Spezies i }
\end{array}
$$

Die Streulichtintensität hängt also linear von der Anzahl der angeregten Moleküle $\mathrm{N}_{i}$ einer bestimmten Spezies und der Intensität des Anregungslichts $\mathrm{I}_{0}$ ab. Im Prinzip bezeichnet der Index i eine bestimmte Schwingung, die von der Molekülstruktur abhängig 
ist. Jedes Molekül, bei dem die betreffende Schwingung auftritt, besitzt demzufolge eine zumindest teilweise gleiche Struktur und wird daher derselben Spezies zugeordnet. Die Detektion des Ramanstreulichts ermöglicht also sowohl die Identifikation der streuenden Molekülspezies als auch eine quantitative Aussage über die Partialdichte bzw. Konzentration. Die Eigenschaften des Detektionssystems werden in der Gleichung durch das Detektionsvolumen, den Raumwinkel der Detektion und eine Konstante ausgedrückt, die weitere Parameter umfasst. 


\section{Planare Ramanstreuung}

In mikrofluidischen Systemen ist der Nachweis bestimmter Molekülspezies von großem Interesse. Die vielfältigen Anwendungen stellen hohe Anforderungen an die verwendeten Messverfahren. Einen Überblick über die breit gefächerten Anwendungen und Detektionstechniken auf dem Feld der Mikrofluidik bieten aktuelle Reviews [5, 49, 71]. Insbesondere zwei große Anwendungsbereiche lassen sich ausmachen. Der erste Bereich umfasst vor allem die medizinische Forschung, die die Entwicklung von Verfahren zum hochsensitiven Nachweis kleinster Konzentrationen vorantreibt. Aufwändige DNA-Analysen werden schrittweise miniaturisiert durchgeführt, so dass sowohl die Analysezeiten als auch die Kosten erheblich gesunken sind [7, 53]. Auch beim Ausbruch des SARS-Virus wurden miniaturisierte Analysesysteme entwickelt [79]. Es ist vorstellbar, dass bereits in wenigen Jahren solche Labs-on-a-chip vor Ort zur Schnellerkennung zum Einsatz kommen. Im zweiten Bereich können alle Anstrengungen zusammengefasst werden, die sich mit der Optimierung und Überwachung mikrofluidischer Mischvorgänge befassen.

Auf dem Feld der Detektion dominieren Fluoreszenzverfahren. Zum Nachweis von Proteinen, Krankheitserregern oder ähnlichem biologischen Probenmaterial kommen speziell entwickelte molekulare Marker zum Einsatz, die an das gesuchte Molekül koppeln [7]. Die Technik erfordert zwar einen teilweise aufwändigen Umweg bei der Suche nach passenden Markern, dafür ist die Detektion der gesuchten Moleküle aufgrund der hohen Ausbeute an Fluoreszenzlicht vergleichsweise einfach. Auch im Fall grundlegender Untersuchungen von Mikromischern ist der Einsatz von Fluoreszenztracern weit verbreitet [11, 24, 26, 43]. In diesem Fall ist die Suche nach einem nutzbaren Tracer einfach, da ein breites Spektrum verschiedener Fluoreszenzfarbstoffe in Frage kommt. Die Verwendung eines Tracers kann aber gerade bei der Beobachtung von Mischungsvorgängen in mikrofluidischen Systemen Probleme aufwerfen. Die treibende Kraft bei der Mischung ist in diesen Dimensionen die Diffusion. Die Diffusionskoeffizienten des Tracers können sich aber zum Teil stark von denen des eigentlich zu beobachtenden Fluides unterscheiden. Zudem wird der Flüssigkeitsantrieb häufig elektrokinetisch durchgeführt, so dass auch das vom Fluid abweichende Masse- zu Ladungsverhältnis eines Tracers zum Problem werden kann. Die Probleme der Fluoreszenztechniken treten bei ramanspektroskopischen Verfahren zu einem großen Teil nicht auf. Ausgenutzt wird bei diesen Techniken die Tatsache, dass jedem polarisierbaren Molekül ein spektraler Fingerabdruck zuzuordnen ist. Die Verwendung zusätzlicher Tracer ist nicht erforderlich, da die gesuchten, bzw. zu beobachtenden Moleküle direkt nachgewiesen werden können. Trotz dieser bestechenden Eigenschaften der Ramanstreuung wird sie nur selten zur Detektion eingesetzt. Der Grund dafür ist der sehr geringe Streuquerschnitt und die damit verbundene sehr geringe Signalintensität. Gerade bei der Untersuchung biologischen Probenmaterials liegen die gesuchten Moleküle zudem oft nur in geringsten Konzentrationen vor. Insbesondere eine Neuentdeckung hat der Ra- 
manspektroskopie als Detektionsverfahren in letzter Zeit Auftrieb gegeben. Durch die Ausnutzung von Verstärkungseffekten an nanostrukturierten Oberflächen (SERS, „Surface enhanced Raman scattering") ist es möglich, die Intensitäten der Ramanstreuung um viele Größenordnungen zu erhöhen [55, 72, 73, 76, 78].

Das hier vorgestellte Messverfahren wurde mit dem Ziel entwickelt, mikrofluidische Mischungsvorgänge zu erfassen. Flüssigkeiten verhalten sich bei den kleinen Abmessungen häufig anders als aus makroskopischen Anwendungen bekannt. Mischungen geschehen ausschließlich auf Basis der Diffusion, da die Strömungen bei den üblicherweise sehr kleinen Reynoldszahlen fast immer laminar verlaufen. Effizientes Mischen ist unerlässlich für eine Vielzahl von Analyse- und Produktionsprozessen, erfordert in mikrofluidischen Systemen aber vollkommen neue Konzepte. Häufig kommen numerische Simulationen zur grundlegenden Optimierung von Mikromischern zum Einsatz [23]. Da bei der Simulation von Gemischbildungsprozessen in mikroskopischen Dimensionen Neuland betreten wird, ist eine Verifikation der Simulationsergebnisse unerlässlich. Ein geschickter Weg, die Effizienz eines binären Mischprozesses zweier Flüssigkeiten abzuschätzen, ist die Nutzung einer Reaktion zwischen den beiden Flüssigkeiten und die Erfassung des Reaktionsproduktes [29]. Die Verteilung der Edukte und Produkte ist in diesem Fall allerdings ebenso unklar, wie die Entstehungsgeschichte dieser Verteilung.

Verfahren, die auf der Nutzung des Ramanspektrums der Fluide beruhen, gibt es wegen der geringen Intensitäten auch auf diesem Gebiet nur in geringer Zahl. Die meisten dieser Verfahren sind auf die punktweise Messung der Ramanspektren ausgelegt und nutzen sie eher unterstützend [54]. In vielen Fällen sind die zu beobachtenden Mikrostömungen stationär, so dass lange Belichtungszeiten eine zweidimensionale Abbildung ermöglichen [74]. Eine weitere Möglichkeit, die Ramanstreuung bildgebend einzusetzen, sind konfokale Ramanmikroskope, mit denen die Strömung punktweise abgerastert werden kann [35, 38, 60]. Der bildgebende Einsatz der Ramanstreuuung erfordert die Verwendung spezieller Filter. Eines der ersten Experimente auf diesem Gebiet nutzt das sehr intensive Ramanspektrum bei UV-Anregung [3]. Die Ramanstreuung wurde in diesem Fall aber nicht zur Unterscheidung verschiedener Flüssigkeiten verwendet. Außerdem wurden bei allen Raman-Untersuchungen Flüssigkeiten eingesetzt, die bei der verwendeten Anregungswellenlänge nicht selbst fluoreszieren. Da laserinduzierte Fluoreszenzemissionen im Gegensatz zu Ramanemissionen in der Regel um einige Größenordnungen höhere Photonenausbeuten aufweisen, wäre eine störungsfreie Detektion des Ramanstreulichts nicht mehr möglich. Die Anforderungen bei der Messung der Konzentration eines Fluides in einer Mischung sind deutlich höher. UV-Strahlung kommt zur Anregung bei der Beobachtung von Gemischbildungsprozessen nicht in Frage, da sich eine Fluoreszenzanregung meist nicht vermeiden lässt. Die resultierenden Ramanintensitäten fallen bei Anregung im sichtbaren oder sogar nahen Infraroten deutlich geringer aus. Zudem können nur geringe Teile des Spektrums genutzt werden, die keine Querempfindlichkeiten zu den anderen Gemischkomponenten zeigen. Dies senkt die Intensitäten des detektierten Ramanstreulichts noch weiter.

Im Rahmen dieser Arbeit wurde eine neue Technik entwickelt, die eine quantitative, hoch zeitaufgelöste, tracerfreie und selektive Detektion einer bestimmten Molekülspezies 


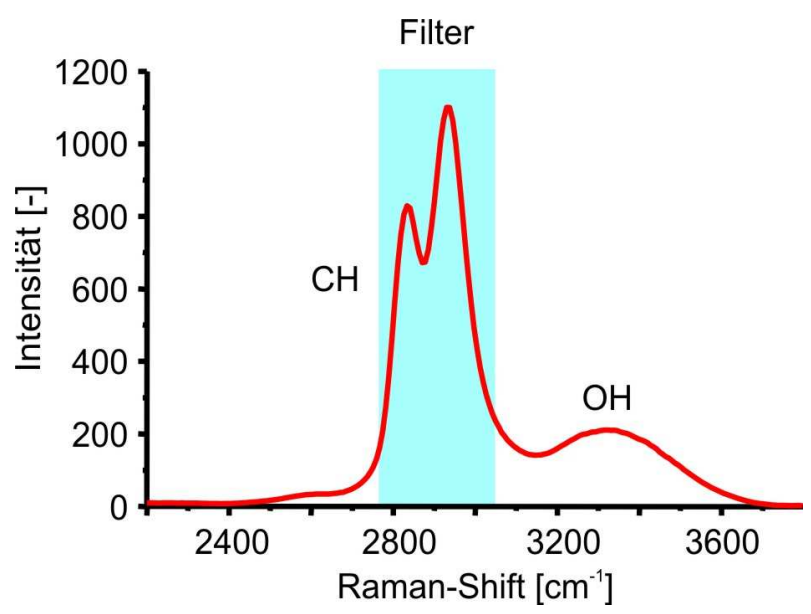

Abb. 5.1.: Messung von Speziesdichteverteilungen durch Planare Ramenstreuung

in einer Mischung mehrerer verschiedener Substanzen ermöglicht. Grundlage der Messtechnik ist die spontane Ramanstreuung. Durch den Einsatz ortsauflösender Spektrometer ist schon seit einigen Jahren eine begrenzte (eindimensionale) räumliche Auflösung zu erzielen. Notwendig ist dazu die Detektion mit einer Kamera. Da auf dem zweidimensionalen Kamerachip aber immer eine Achse für das Spektrum reserviert ist, ist die Aufzeichnung einer zweidimensionalen Verteilung mit dieser Methode nicht möglich. Bei der Messung planarer Ramanstreuung wird statt des Spektrometers ein Filter vor der Kamera verwendet, was eine Aufnahme der zweidimensionalen Konzentrationsverteilung möglich macht. Ein vergleichbares Verfahren wurde zeitgleich auch von einer weiteren Arbeitsgruppe entwickelt [44], jedoch nicht auf mikroluidische Gemischbildungsvorgänge angewandt.

\subsection{Prinzip der Messtechnik}

Im letzte Kapitel wurde beschrieben, dass jeder Molekülspezies eine Art spektraler Fingerabdruck zugeordnet werden kann. Zur Aufnahme der zweidimensionalen Verteilung einer Spezies mit einer Kamera muss ein charakteristischer Spekralbereich dieses Fingerabdrucks selektiert werden, in dem keine Querempfindlichkeiten zu anderen in der Mischung vorhandenen Molekülspezies auftreten. Auf diese Weise ist es möglich, im Idealfall nur das Ramanstreulicht einer bestimmten Spezies in einer Mischung zu detektieren. In Abbildung 5.1 ist ein Teil des Ramanspektrums von Ethanol dargestellt. Im abgebildeten Spektrum sind mehrere Intensitätsmaxima zu erkennen. Diesen lassen sich bestimmte molekulare Schwingungen zuordnen. Das höchste Maximum ist dabei auf die Streckschwingung der CH-Gruppen zurückzuführen. Daneben existiert noch ein deutlich breiteres und schwächer ausgeprägtes Maximum der OH-Schwingung. Gekennzeichnet ist außerdem der ideale Transmissionsbereich des zu verwendenden optischen Filters zur 
Detektion von Ethanol anhand der charakteristischen CH-Schwingung.

Die Auswahl der CH-Bande zur Detektion eines bestimmten Kohlenwasserstoffes ist jedoch in vielen Fällen ungeschickt, da bei vielen Mischungen mehrere organische Stoffe vertreten sind, die jeweils selbst eine oder mehrere CH-Gruppen aufweisen und dementsprechend im selben Spektralbereich Ramanstreulicht aussenden. Im Einzelfall muss die Auswahl des Ramanfilters an das konkrete Fluidsystem angepasst werden. Der Filter in der Abbildung würde zum Beispiel bei einer Mischung von Ethanol und Wasser Sinn machen. In diesem Fall würde das Wasser nur im Bereich der OH-Bande Ramanstreulicht emittieren. Die OH-Bande ist ist jedoch auch beim Ethanol vorhanden und in der Abbildung zu sehen. Da diese Bande vom vorgeschlagenen Filter nicht erfasst wird, würde nur Licht selektiert, welches allein vom Ethanol emittiert wird.

Eine weitere wichtige Eigenschaft der Ramanstreuung ist, dass die abgestrahlte Intensität direkt proportional zur Teilchenzahl im Messvolumen ist. Auf dieser Grundlage sind quantitative Messungen der Spezieskonzentration möglich. Für die Konzentrationsmessung ist zuvor eine Kalibration notwendig.

\subsection{Speziesverteilungen im flachen Mikromischer}

Das Messverfahren wurde zunächst auf den flachen Mikromischer angewendet, der in Kapitel 2.1 beschrieben wurde und bereits für die Geschwindigkeitsmessungen zum Einsatz kam. Der experimentelle Aufbau, sowie die verwendete Mischergeometrie sind in Abbildung 5.2 dargestellt. Der Mikromischer wird in seiner einfachsten Form eingesetzt, das bedeutet die beiden Flüssigkeiten fließen über eine Y-Verbindung zusammen und durch die Mischkammer zum Abfluss. Die Seitenkanäle bleiben bei diesem Experiment ungenutzt. Für die Anregung des Ramanstreulichts kommt ein frequenzverdoppelter Nd:YAGLaser mit einer Wellenlänge von $532 \mathrm{~nm}$ zum Einsatz. Der Laserstrahl wird durch eine Streuscheibe aufgeweitet und gleichzeitig homogenisiert. Das Anregungslicht leuchtet den Mikromischer vollständig aus. Der Ramanfilter selektiert das Streulicht der CH-Bande zur Detektion von Ethanol und ein zusätzlicher Filter sorgt dafür, dass das Anregungslicht unterdrückt wird. Das Ramanstreulicht wird von einer intensivierten CCD-Kamera verstärkt und aufgenommen.

Da die Ramanstreuung ein sehr lichtschwacher Prozess ist, ist die Aufnahme von ungemittelten Einzelbildern sehr schwierig. Die betrachtete Strömung ist stationär und die Mischung kann bei der geringen Reynoldszahl (bei den genutzten Durchflussraten zwischen 0,5 und 5) nur rein diffusiv stattfinden, so dass sich ein statisches Bild ergibt. Dies ermöglicht bei zu geringen Intensitäten eine Mittelung, bzw. Aufsummierung mehrerer Aufnahmen. Abbildung 5.3 zeigt drei Aufnahmen der durchströmten Mischkammer. Oben befindet sich das Ethanol und unten das Wasser. In allen drei Fällen gilt dieselbe Intensitätsskala. Für jede Aufnahme wurde eine unterschiedliche Anzahl von Laserpulsen summiert. Die Lichtverstärkung der Kamera wurde bei allen Aufnahmen auf das gleiche hohe Level eingestellt, so dass die Bedingungen vergleichbar sind. Trotz der hohen Verstärkung ist in der Aufnahme a mit einem einzelnen Laserpuls nahezu kein Ramanstreulicht detektierbar. Bei Summierung von zehn Pulsen lassen sich die ersten 


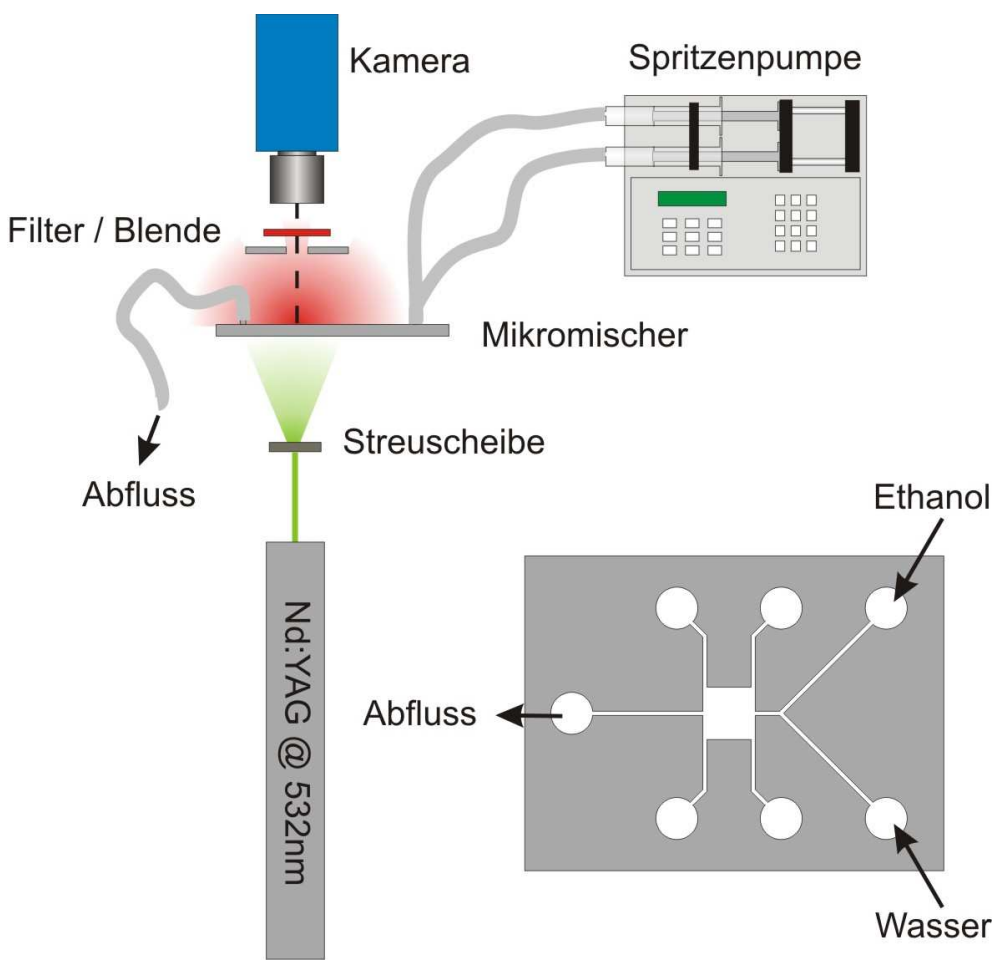

Abb. 5.2.: Experimenteller Aufbau zur Aufnahme planarer Ramanstreuung

Strukturen erkennen und bei Summierung von 100 Laserpulsen ist die Verteilung des Ethanol deutlich sichtbar. Da die Anregungsfrequenz durch den Laser auf $10 \mathrm{~Hz}$ fixiert ist, liegt die gesamte Belichtungszeit bei dieser Aufnahme bereits bei etwa 10 Sekunden.

Mit diesen ersten Aufnahmen konnte gezeigt werden, dass die planare Messung von Speziesdichteverteilungen mittels spontaner Ramanstreuung möglich ist. Quantitative Aussagungen zu den Konzentrationen der beteiligten Fluide sind aufgrund der geringen Intensitäten schwierig und in diesem Stadium nicht durchgeführt worden. Die Summierung einer großen Anzahl von Laserpulsen führt zu höheren Intensitäten jedoch auch zu deutlich höheren Belichtungszeiten. Selbst Mischprozesse mit nur sehr geringer Dynamik lassen sich mit dieser Zeitauflösung kaum untersuchen. Die Aufnahmen würden durch die hohe Belichtungszeit zu stark verwischen. Eine deutliche Steigerung der aufgenommenen Intensitäten, bzw. eine Verkürzung der Belichtungszeiten ist notwendig.

\subsection{Steigerung der Intensitäten}

Damit quantitative Messungen von Speziesdichteverteilungen erfolgreich sein können, ist es notwendig, das Messsystem hinsichtlich einer größeren Photonenausbeute zu optimieren. Die folgenden Bereiche kommen für eine Optimierung der Intensitätsausbeute in Frage. 


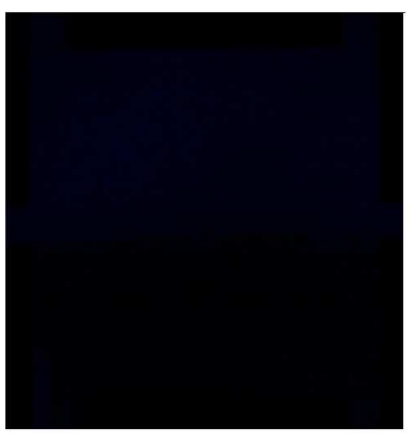

a)

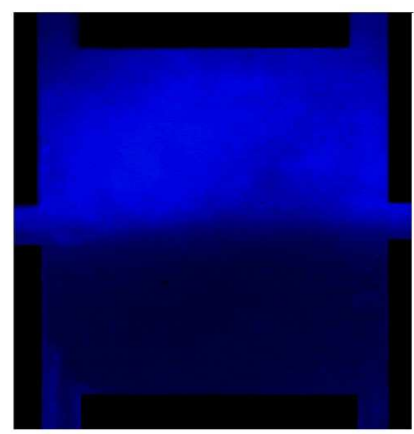

b)

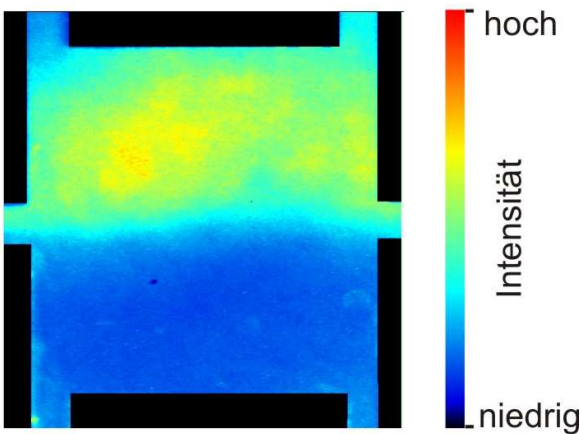

c)

Abb. 5.3.: Ramanaufnahme der durchströmten Mischkammer, oben Ethanol, unten Wasser a) einzelner Laserpuls b) zehn Laserpulse summiert c) 100 Laserpulse summiert

1. die Anregung

2. der Ramanprozess

3. das Detektionssystem

Die Anregung kann zum Beispiel durch höhere Pulsenergien des Lasers verstärkt werden. Die Detektionsoptiken sollten eine möglichst hohe numerische Apertur aufweisen und das Detektionssystem eine hohe Quantenausbeute. Der Ramanprozess selbst lässt sich zwar im wörtlichen Sinne nicht optimieren, es ist aber möglich, den Aufbau an die Eigenschaften des Ramanstreuprozesses anzupassen. Im Folgenden soll auf die verschiedenen Optimierungsmöglichkeiten eingegangen werden und es soll abgeschätzt werden, wie wirkungsvoll sie sind.

\subsubsection{Anregung}

Die sehr geringen Ramansteuquerschnitte machen von vorn herein hohe Anregungsenergien notwendig. Bei einem gepulsten Nd:YAG-Laser mit einer Pulslänge von etwa 6 ns bedeuten hohe Pulsenergien enorme Laserleistungen. Wenn die obere Leistungsgrenze des verwendeten Lasers noch nicht erreicht ist, kann man den Anregungspuls weiter verstärken, um ein höheres Ramansignal zu erhalten. Dabei dürfen die Zerstörschwellen der verwendeten Materialien allerdings nicht überschritten werden und der Energieeintrag in die untersuchten Fluide muss so gering bleiben, dass keine Beeinflussung durch den Messvorgang auftritt. Damit die kritischen Schwellen der Leistung nicht überschritten werden, kann die zeitliche Verteilung der Energie ausgedehnt werden. Eine entsprechende Möglichkeit ist die Summation der Ramanintensitäten mehrerer Anregungspulse. Wie im vorigen Abschnitt gezeigt wurde, ist dies aufgrund der signifikant höheren Belichtungszeiten, die sich bei dem zur Verfügung stehenden 10 Hz-Laser ergeben, jedoch unerwünscht. Ebenfalls denkbar ist die Verwendung eines kontinuierlich arbeitenden Lasers. Aufgrund 
der wesentlich geringeren Leistung eines derartigen Lasers würde sich wiederum eine sehr hohe Belichtungszeit ergeben, um eine vergleichbare Intensität des Ramanstreulichts zu erhalten. Eine kurze Rechnung soll das Problem verdeutlichen. Die maximale Belichtungszeit wird von der Strömungsgeschwindigkeit der Flüssigkeit bestimmt. Damit eine Abbildung scharf bleibt, darf sich die Flüssigkeit während der Belichtung nur sehr wenig bewegen. Ein sehr gutes Maß für die maximal zulässige Bewegung kann aus der Abbildung auf den Kamerachip berechnet werden. Als Grenzwert für die Bewegung wird ein halber Bilpunkt festgelegt. Die Auflösung des später verwendeten Kamerachips (vgl. Kapitel 5.4) beträgt $512 \times 512$ Bildpunkte. Der beobachtete Bereich ist $10 \mathrm{~mm}$ breit und wird auf etwa 500 Bildpunkte abgebildet. Damit ergibt sich eine maximal akzeptable Bewegung der Flüssigkeit von $10 \mu \mathrm{m}$ während der Belichtungszeit. Die Strömungsgeschwindigkeiten ergeben sich aus den vorgegebenen Volumenflüssen. Die höchsten Geschwindigkeiten treten in den schmalen Zulaufkanälen $(1 \times 1 \mathrm{~mm})$ auf. Im weiteren Verlauf weitet sich die Geometrie auf eine Querschnittsfläche von $10 \times 10 \mathrm{~mm}$ auf. Hier sind tendenziell geringere Geschwindigkeiten zu erwarten. Der höchste eingestellte Durchfluss von etwa $100 \mathrm{ml} / \mathrm{min}$ pro Flüssigkeit teilt sich auf jeweils acht Zulaufkanäle auf. Es ergibt sich eine gerundete mittlere Strömungsgeschwindigkeit von 208 mm/s. Die maximale Strömungsgeschwindigkeit läge bei einer Rohrströmung um einen Faktor zwei höher als die mittlere Strömungsgeschwindigkeit. Hier wird für die weiteren Rechnungsschritte eine maximale Strömungsgeschwindigkeit von $400 \mathrm{~mm} / \mathrm{s}$ verwendet. Aus dieser Strömungsgeschwindigkeit und der zuvor berechneten maximalen Bewegung ergibt sich eine maximale Belichtungszeit von $25 \mu \mathrm{s}$. Ein einzelner Laserpuls des verwendeten Nd:YAG-Lasers unterschreitet diese Schwelle mit einer Pulsdauer von ca. 6 ns bei weitem. Die Verwendung mehrerer Laserpulse ist bei einer Repetitionsrate von $10 \mathrm{~Hz}$ nach diesen Überlegungen jedoch ausgeschlossen. Wenn nur zwei Pulse eingesetzt würden, läge die effektive Belichtungszeit mit $100 \mathrm{~ms} 4.000$ fach zu hoch. Selbst ein hochrepetierender Laser wäre bei $100 \mathrm{kHz}$ lediglich in der Lage, zwei Pulse in der erlaubten Belichtungszeit unterzubringen. Der Einsatz mehrerer Laserpulse zur Erhöhung der eingestrahlten Energie ist also nicht sinnvoll durchführbar. Ein typischer cw-Laser erreicht eine maximale Leistung von $5 \mathrm{~W}$. In der genannten Belichtungszeit könnten daher 0,125 mJ das Beobachtungsvolumen erreichen. Verglichen damit erreicht der Nd:YAG-Laser eine 2.000 mal höhere Energie in einem Puls, nämlich maximal 250 mJ. Der Einsatz des cw-Lasers ist folglich ebenfalls nicht sinnvoll.

Eine realisierbare Möglichkeit, die hohe Pulsenergie auf eine größere Zeitspanne zu verteilen, ist der Einsatz einer Verzögerungsstrecke. Dabei wird der Laserpuls mittels Strahlteilern mehrfach aufgeteilt, die Teilstrahlen über verschieden lange Verzögerungsstrecken geschickt und wieder in sich selbst zurückgeführt, so dass die Pulslänge erhöht wird. Signifikante Steigerungen der Pulsdauer um beispielsweise einen Faktor 10 sind dabei allerdings nur sehr schwer zu erzielen, da dafür größere Verzögerungsstrecken benötigt würden. Diese lassen sich mit vertretbarem Aufwand nur durch Mehrfachreflektionen erreichen, was immer auch Energieverluste bedeutet. Diese Verluste sorgen zudem dafür, dass die Pulsenergie des Lasers erheblich gesteigert werden müsste um einen längeren Puls derselben Energie zu erzeugen, als ohne Pulsverlängerung emittiert würde. Auch 


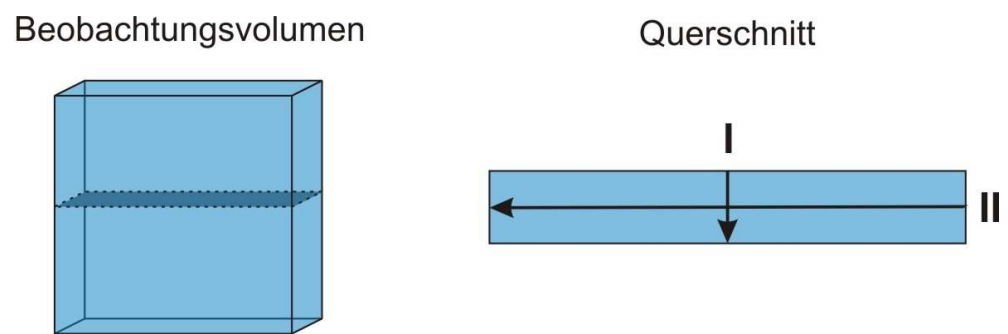

Abb. 5.4.: Steigerung der Intensitätsausbeute durch geänderte Anregung. Der Querschnitt rechts zeigt, dass ein Photon bei Einstrahlrichtung II eine deutlich längere Strecke im Medium zurücklegt als bei Richtung I.

diese Maßnahme würde im Experiment zu deutlich erhöhtem Aufwand führen, die möglichen Anregungsenergien aber nur geringfügig steigern.

Statt der zeitlichen Verteilung des anregenden Laserlichts kann auch die räumliche Aufteilung geändert werden. Dies führt bei einer geschickten Wahl der Anordnung der Komponenten des Aufbaus ebenfalls zu höherer Intensität des Ramanstreulichts, obwohl ein gleich starker Laserpuls verwendet wird. Im Folgenden wurde zu einem neuen Aufbau gewechselt, bei dem das anregende Laserlicht zu einem Laser Lichtband geformt wird, mit dem eine Ebene im Untersuchungsobjekt beleuchtet wird. Die Änderungen bei der Anregung führen zu einer deutlichen Steigerung der Intensitätsausbeute. Dies ist einfach verständlich, wenn man Abbildung 5.4 betrachtet. Im linken Teil ist das Beobachtungsvolumen mit einem beliebigen Querschnitt skizziert. Wenn man diesen Querschnitt in der vergrößerten Aufsicht rechts betrachtet, findet man zwei mögliche optische Wege I und II. Ein anregendes Photon, dass sich in Anregungsrichtung II bewegt, muss eine deutlich längere Strecke im Beobachtungsvolumen durchqueren als eines, dass sich in Richtung I bewegt. Die Wahrscheinlichkeit einer Absorbtion und folgender Emission eines Ramanphotons wird also um den Faktor vergrößert, um den Weg II länger als Weg I ist. Diese Überlegung gilt für jedes einzelne Lichtquant. Die Intensitätsausbeute des Ramanprozesses wird durch diese Maßnahme also erheblich verbessert, obwohl die Laserleistung der Anregung nicht verändert wurde. Im Falle der diffusen Auflichtbeleuchtung beim flachen Mikromischer, wie er in Abbildung $5.2 \mathrm{zu}$ sehen ist, durchquert jedes Photon $500 \mu \mathrm{m}$ des Fluides. Wenn man einen Laserstrahl gleicher Pulsenergie so formt, dass man ihn von der Seite in die Mischkammer einkoppeln kann und dabei exakt dasselbe Volumen ausleuchtet, dann durchquert jedes Photon eine Strecke von $5 \mathrm{~mm}$. Die Effizienz der Anregung steigt durch die längere Strecke um einen Faktor $5 \mathrm{~mm} / 500 \mu \mathrm{m}=10$. Problematisch ist bei diesem Mikromischer die Einkopplung des Laserstrahls, da die Seitenwände nicht transparent sind und auch nur unter sehr hohem Aufwand aus geeigneten transparenten Materialien hergestellt werden könnten. Der zweite vorgestellte Mikromischer ist in dieser Hinsicht überlegen, da er in alle Richtungen ideal transparente Begrenzungen aufweist. Aufgrund dieses Vorteils wird im Folgenden dieser Multilaminationsmischer für die weitere Optimierung des Ramanmessaufbaus eingesetzt. Die Strahlformung zu einem Laser 
Lichtband bietet, verglichen mit voluminöser Beleuchtung, den zusätzlichen Vorteil, dass man eine definierte Ebene im Mischer auswählen kann.

\subsubsection{Ramanprozess}

Die Intensität der resultierenden Ramanstreuung ist von mehreren Parametern abhängig. Aus Gleichung 4.10 können die wichtigsten Abhängigkeiten abgelesen werden. Die Intensität $I_{i}$ des Ramamsignals einer Spezies ist proportional zur Intensität der Ausgangsstrahlung $\mathrm{I}_{0}$. Dieser Zusammenhang wurde im vorangegangenen Abschnitt zur Optimierung genutzt. Zudem besteht eine direkte Proportionalität zur Zahl der Moleküle $\mathrm{N}_{i}$ im Anregungsvolumen. Aufgrund dieser Proportionalität können die aufgenommenen Intensitäten für eine quantitative Bestimmung der Spezieskonzentrationen genutzt werden.

Aus Gleichung 4.9 ist ersichtlich, dass auch die Frequenz des Anregungslichts $\omega$ eine wichtige Rolle bei der Intensitätsausbeute spielt. Sie ist in der Gleichung in vierter Potenz enthalten, das bedeutet, dass kurzwelligeres Licht zu erheblich mehr Ramanstreulicht führt. Eine Halbierung der Wellenlänge zieht eine Versechzehnfachung des resultierenden Ramanstreulichts nach sich. Trotz dieser Tatsache werden nahezu alle Messverfahren, die auf der spontanen Ramanstreuung beruhen, im Bereich des sichtbaren oder sogar infraroten Lichts durchgeführt. Der Grund dafür ist die vermehrte gleichzeitige Anregung von Fluoreszenzen. Je weiter man sich zu kurzen UV-Wellenlängen bewegt, desto mehr Fluoreszenzlicht überlagert das gewünschte Ramanspektrum. Die Intensität des Fluoreszenzlichts ist meist um viele Größenordnungen höher als die des relevanten Ramanstreulichts. Sinnvolle Messungen sind in diesem Fall nicht mehr möglich. Testmessungen mit einem UV-Excimerlaser bei $355 \mathrm{~nm}$ bestätigten diese Überlegungen. Die entstandenen Aufnahmen sind deutlich überstrahlt. Eine Anregung mit einem Nd:YAG-Laser bei $532 \mathrm{~nm}$ führte beim Messsystem dagegen nicht zu Problemen.

Auch die Polarisation des Anregungslichts spielt eine Rolle für die resultierenden Intensitäten. In Kombination mit der Polarisierbarkeit der betrachteten Spezies können sich Intensitätsunterschiede bei Beobachtung aus verschiedenen Richtungen ergeben. Dieser Zusammenhang wurde unter anderem in Abbildung 4.2 verdeutlicht. Bei den Überlegungen, die in diesem Zusammenhang angestellt wurden, wurde aber bisher von einzelnen Molekülen ausgegangen. Tatsächlich werden aber für gewöhnlich große Mengen von Molekülen betrachtet. Die Verteilung und Orientierung der Moleküle kann sich erheblich unterscheiden. Kristalle sind gleichmäßig strukturiert, so dass auch die Polarisierbarkeiten ausgerichtet vorliegen. Flüssigkeiten und Gase dagegen enthalten eine statistische Verteilung aller möglichen Orientierungen der Moleküle. So unterschiedlich die verschiedenen betrachteten Proben sind, so sehr kann sich auch der Einfluss der Polarisation des Anregungslichts unterscheiden. Im gegebenen Fall werden Flüssigkeiten mit linear polarisiertem Laserlicht angeregt. Am Beispiel des zweiatomigen Moleküls in Abbildung 4.2 ist ersichtlich, dass es ungeschickt wäre, die Detektion in Richtung der Polarisation des Anregungslichts durchzuführen, da in diesem Fall nur die vektoriellen Komponenten, die entgegen der Polarisation der Anregung liegen, detektiert werden könnten. Effizienter ist eine Detektion senkrecht zur Polarisation des Anregungslichts. Üblicherweise werden 
Detektionsrichtungen unter $0^{\circ}, 90^{\circ}$ oder $180^{\circ}$ zur Richtung des einfallenden Anregungslichts verwendet. Zur Minimierung von detektiertem Anregungslicht ist eine Detektion unter $90^{\circ} \mathrm{zu}$ bevorzugen, da in diesem Fall das meiste Anregungslicht die Detektionsoptiken nicht erreichen kann. Lediglich die Rayleighstreuung stellt nun noch einen geringen Anteil, der mit Filtern entfernt werden muss.

\subsubsection{Optiken und Detektion}

Auch der Detektionszweig kann auf einen möglichst hohen Intensitätswert optimiert werden. Dafür gibt es verschiedene Möglichkeiten. Das Ramanstreulicht durchquert auf dem Weg zum Kamerachip zunächst den Ramanfilter, sowie die abbildenden Optiken. Der Filter muss nicht nur spektral auf die interessierende Molekülspezies angepasst werden, sondern außerdem hohe Transmissionswerte im relevanten Bereich aufweisen. Je besser der Filter, desto mehr Ramanlicht erreicht das Detektionssystem. Die Linsen der abbildenden Optik sollten so viel Licht wie möglich einfangen, dies ist bei einer hohen numerischen Apertur der Fall. Die Optiken sollten also möglichst groß sein und nah am Messvolumen positioniert werden. Die Tiefenschärfe, die normalerweise durch eine Verringerung der freien Apertur der Linsen vergrößert würde, spielt hier keine Rolle, da die Dicke der Messebene durch die Dicke des Laser Lichtbandes vorgegeben wird.

Die verwendete Kamera hat ebenfalls einen wichtigen Anteil an einer effizienten Detektion. Das Kamerasystem muss in der Lage sein, möglichst viele eintreffende Photonen zu registrieren. Zudem ist bei geringsten Intensitäten ein verstärkendes System sinnvoll. Wichtig ist die Detektion möglichst vieler Ramanphotonen und ein geringes Eigenrauschen des verwendeten Kamerasystems.

\subsection{Verbessertes Detektionssystem}

Die verschiedenen Optimierungsschritte führten zu einem erheblich verbesserten Aufbau. Dieser ist in Abbildung 5.5 dargestellt. Die Überlegungen aus den letzten Abschnitten wurden dabei verwendet, um eine möglichst effiziente Ausnutzung des Anregungslichts, eine optimierte Umwandlung von Ramanphotonen in elektrische Signale zu garantieren. Die Messungen wurden anstatt mit dem flachen Mischersystem mit dem Multilaminationsmischer durchgeführt. Dieser ist im Bereich der Mischstrecke vollständig optisch zugänglich und erlaubt daher die Verwendung eines rechtwinkligen Aufbaus. Der Laserstrahl wird zur Anregung zu einem Laser Lichtband geformt. Die Strahlformung geschieht durch die Kombination von zwei Zylinderlinsenarrays und einer Fourierlinse. Die Anordnung sorgt neben einem möglichst flachen Lichtband (ca. $500 \mu \mathrm{m}$ ) auch für eine homogene Ausleuchtung der beobachteten Fläche. Das Lichtband selektiert eine definierte Beobachtungsebene in der Mischstrecke. Der Mikromischer lässt sich mit Mikrometertrieben in alle Richtungen verfahren, so dass der Messbereich definiert in der Mischstrecke positioniert werden kann.

Der Anregungszweig wurde so eingestellt, dass die senkrechte Polarisation des Lasers erhalten bleibt, damit bei der Detektion unter einem $90^{\circ}$-Winkel zur Anregung möglichst 


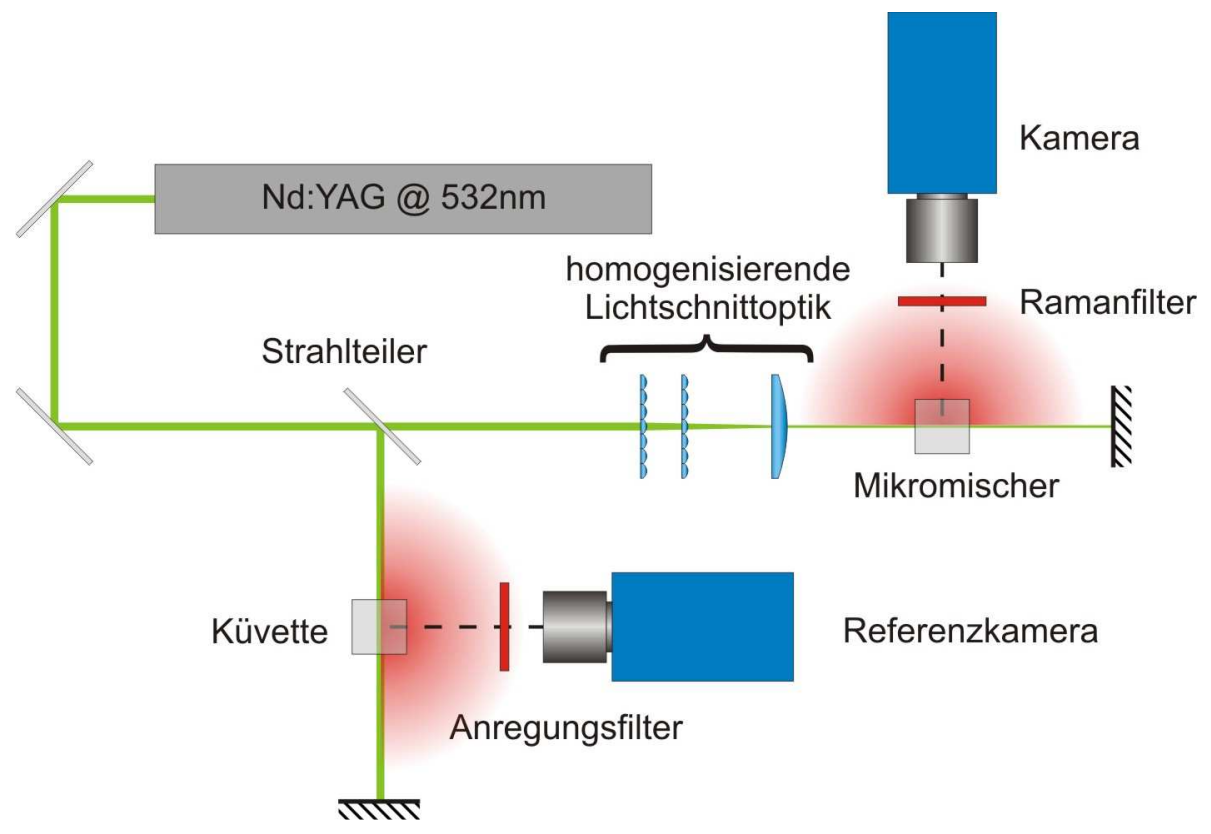

Abb. 5.5.: Optimierter Experimenteller Aufbau zur Aufnahme planarer Ramanstreuung

viel Ramanstreulicht die Kamera erreicht. Die rechtwinklige Anordnung sorgt weiterhin dafür, dass kaum Laserlicht in den Detektionsarm fällt. Dies ist sehr wichtig, da die eingesetzten Ramanfilter allein nicht in der Lage wären, direkt auftreffendes Anregungslicht ausreichend zu blockieren. Zusätzliche Filter, die diese Aufgabe übernehmen könnten würden auch die relevanten Ramanlinien teilweise unterdrücken und somit die Detektion erschweren. Die in dieser Arbeit verwendeten Flüssigkeiten sind überwiegend Wasser und Ethanol, da sie einfach verfügbar und relativ ungefährlich sind. Zudem ist das Ramanspektrum von Ethanol im Bereich der CH-Streckschwingung besonders intensiv und daher verhältnismäßig einfach zu detektieren. Zur Detektion wird ein Filter verwendet, der das Licht der CH-Bande spektral selektiert, während sowohl das Anregungslicht als auch das Ramanstreulicht weiterer Banden abgeblockt wird.

Zur Detektion wird eine EMCCD-Kamera (Ixon, DU897D) der Firma Andor verwendet. Diese Kamera erzielt Quanteneffizienzen von maximal $93 \%$ und ist allein durch diese Eigenschaft der bisher verwendeten ICCD mit einer maximalen Quanteneffizienz von ca. $12 \%$ weit überlegen. Zudem ist das Eigenrauschen dieser Kamera durch eine Peltierkühlung bis zu $-100^{\circ} \mathrm{C}$ so weit minimiert, dass bei aktivierter Verstärkung die Detektion schwächster Signale möglich ist. Auf die verwendeten Ramanfilter wird in den nächsten Abschnitten gesondert eingegangen, da sie eine der wichtigsten Komponenten des Messverfahrens darstellen. Durch den $90^{\circ}$-Aufbau ist bei sauberen Flüssigkeiten, also ohne die direkte Reflektion von anregendem Laserlicht an Staubteilchen oder ähnlichem, der jeweilige Ramanfilter ausreichend zur Unterdrückung des Anregungslichts.

Zusätzlich zu den Maßnahmen, die für eine Verstärkung des detektierten Signals, 
durchgeführt wurden, kamen weitere Verbesserungen zum Einsatz. Diese wurden im Verlauf der Messungen notwendig, um eine genaue Quantifizierung der gemessenen Signalintensitäten zu ermöglichen. Der Einbau eines Referenzarmes soll dabei Schwankungen der Laserleistung zeitgleich mit der Aufnahme der Speziesverteilungen detektieren. Mit diesen zusätzlichen Daten ist es möglich, die Schwankungen größtenteils zu eliminieren. Für den Referenzarm wurde ein Strahlteiler in den Strahlengang eingefügt, der $60 \%$ der Ausgangsstrahlung in eine Küvette umleitet. Die Energie, die durch die Auskopplung im Detektionsarm fehlt, wurde durch eine Erhöhung der Ausgangsenergie des Lasers kompensiert. Im Refenrenzarm steht durch den Strahlteiler die anderhalbfache Energie des Detektionsarmes zur Verfügung. Die höheren Energien sind notwendig, da in der zweiten Küvette ebenfall Ramanstrahlung angeregt wird, die mit einer nicht verstärkten Kamera (Flowmaster 3, Fa. Lavision) detektiert wird. In der Küvette befindet sich reines Ethanol, damit maximale Intensitäten detektiert werden können. Der Filter vor der Kamera filtert lediglich das Anregungslicht heraus. Dadurch erreicht das komplette Ethanol-Ramanspektrum und nicht nur das Ramanstreulicht der CH-Bande die Kamera, sodass eine zusätzliche Intensitätserhöhung generiert wird. Weiterhin wird auf eine Strahlformung verzichtet, was zu geringeren Verlusten bei der Anregung führt. Durch diese Maßnahmen ist das Ramanstreulicht im Referenzarm erheblich stärker als im Beobachtungsarm. Die detektierten Intensitäten werden zusätzlich über einen Bereich des Kamerachips summiert, dadurch erhält man ein ausreichend hohes Referenzsignal trotz fehlender Bildverstärkung. Auf die zeitaufgelöste Korrektur der Laserschwankungen wird in Kapitel 5.7.2 eingegangen.

\subsection{Ramanfilter}

Eine der wichtigsten Komponenten für zweidimensionale Messungen der Ramanstreuung bestimmter Moleküspezies ist der Ramanfilter. Dieser Filter soll das Ramanstreulicht einer bestimmten Spezies selektieren, ohne dabei das Streulicht einer weiteren Spezies zu erfassen. Komplexere organische Fluide liefern häufig keine charakteristischen Ramanbanden mehr, sondern einen quasi kontinuierlichen Intensitätsverlauf über einen breiten Wellenlängenbereich. Die Moleküle bestehen aus einer so großen Menge von unterschiedlichen Atomen und Atomsorten, dass die hohe Zahl von Bindungen und demzufolge energetisch unterschiedlichen Molekülschwingungen zum Überlappen der einzelnen Ramanbanden dieser Schwingungen führt. Die Trennung zweier solcher Spezies ist bei ausreichendem Intensitätsunterschied zwar ebenfalls möglich, darauf soll aber erst später eingegangen werden. Zunächst wird davon ausgegangen, dass die Spektren der auftretenden Spezies sich ausreichend unterscheiden, um eine Ramanbande der zu vermessenden Spezies zu finden, die keine Querempfindlichkeiten zu den restlichen Spezies aufweist. Die Anforderungen an den Filter sind dadurch sehr hoch. Die Ramanspektren der meisten Stoffe überlappen vielfach, wobei die charakteristischen Ramanbanden sehr nah beieinander liegen. Der Filter muss also ausreichend schmalbandig und steilkantig sein. Er sollte zudem gestreutes Anregungslicht ausreichend unterdrücken.

Unterschiedlichste Anforderungen auf den Gebieten der optischen Messtechnik ha- 
ben eine hohe Vielfalt an Filtern hervorgebracht. Um die notwendigen Eigenschaften zu erhalten werden dabei mehrere verschiedene Produktionsprozesse verwendet. Einfache und günstige Farbglasfilter werden vor allem in der Fotografie eingesetzt. Holographische Filter sorgen mit ihren enorm hohen optischen Dichten für selektive schmalbandige Unterdrückung von Laserstrahlung. Die vielseitigsten Möglichkeiten bieten beschichtete Interferenzfilter. Die Eigenschaften dieses Filtertyps lassen sich nahezu beliebig designen und auf die Anforderungen der jeweiligen Anwendung anpassen. Eine relativ neue Entwicklung sind spezielle durchstimmbare Filter auf akusto-optischer Basis, mit denen zweidimensionale Abbildungen unverfälscht möglich sind.

Die Qualitäten der eingesetzten Filter ergeben sich häufig aus ihrem Einsatzgebiet. Farbglasfilter sind für gewöhnlich weder schmalbandig noch steilkantig, kommen also als Ramanfilter nicht in Frage. Filter, die zur gezielten Selektion oder Unterdrückung von Laserlicht verwendet werden, erfüllen die gleichen Voraussetzungen wie sie an einen Ramanfilter gestellt werden. Besonders holographische Filter erreichen ausreichend hohe optische Dichten um auch direktes Laserlicht zu unterdrücken. Aber auch Interferenzfilter erreichen die notwendigen optischen Dichten, um in diesem Bereich eingesetzt zu werden. Da Laser mit den unterschiedlichsten Wellenlängen existieren, sind auch die gängigen Filter in einem weiten Spektralbereich erhältlich. Ein weiteres Einsatzgebiet ist die biologische Forschung. Hier werden in großem Stil Fluoreszenzfiltersets eingesetzt, mit denen zum Beispiel mikroskopische Untersuchungen der Vorgänge in Zellen durchgeführt werden. Für diese Anwendungen werden ebenfalls steilkantige Interferenzfilter eingesetzt. Viele Filter sind jedoch nicht schmalbandig genug. Da das Feld der Fluoreszenzmikroskopie einen beachtlichen Anteil auf dem Markt optischer Filter ausmacht, exisiteren trotzdem besonders viele Möglichkeiten, passende Filter für die Ramanmessungen zu finden. Dies gilt insbesondere für den sichtbaren Spektralbereich. Trotz dieser umfangreichen nutzbaren Ressourcen an kommerziell erhältlichen Filtern ist es oft nicht möglich, einen Filter zu finden, der die Anforderungen an eine bestimmte Messaufgabe erfüllt. Für gewöhnlich werden die Anforderungen aber zumindest teilweise erfüllt. Eine besondere Eigenschaft von Interferenzfiltern kann helfen, die Probleme zu mindern oder gar zu beseitigen. Üblicherweise werden Filter senkrecht zur optischen Achse in den Strahlengang eingebracht. Verdreht man die Stellung eines Interferenzfilters ein wenig gegen die optische Achse, so verändert sich sein Transmissionsspektrum. Der Transmissionsbereich eines Bandpassfilters verschiebt sich zu längeren Wellenlängen. Auf diese Weise können auch Filter verwendet werden, die neben dem eigentlich interessanten Spektralbereich liegen.

Im Folgenden sollen die genanten Filtertypen und Techniken in verschiedenen Szenarien eingesetzt werden, bei denen unterschiedliche Fluide gemischt werden. Die Anforderungen werden dabei durch Variation der gemischten Flüssigkeiten verändert. Zunächst wird eine einfache Mischung von Wasser und Ethanol vorgegeben, die für den Aufbau des Messsystems ideal ist. Desweiteren soll ein Filter für eine Mischung gefunden werden, bei der die Detektion aus spektraler Sicht deutlich schwieriger ist. Zuletzt wird auf die mögliche Verwendung eines akusto-optischen Transmissionsfilters eingegangen (AOTF). 


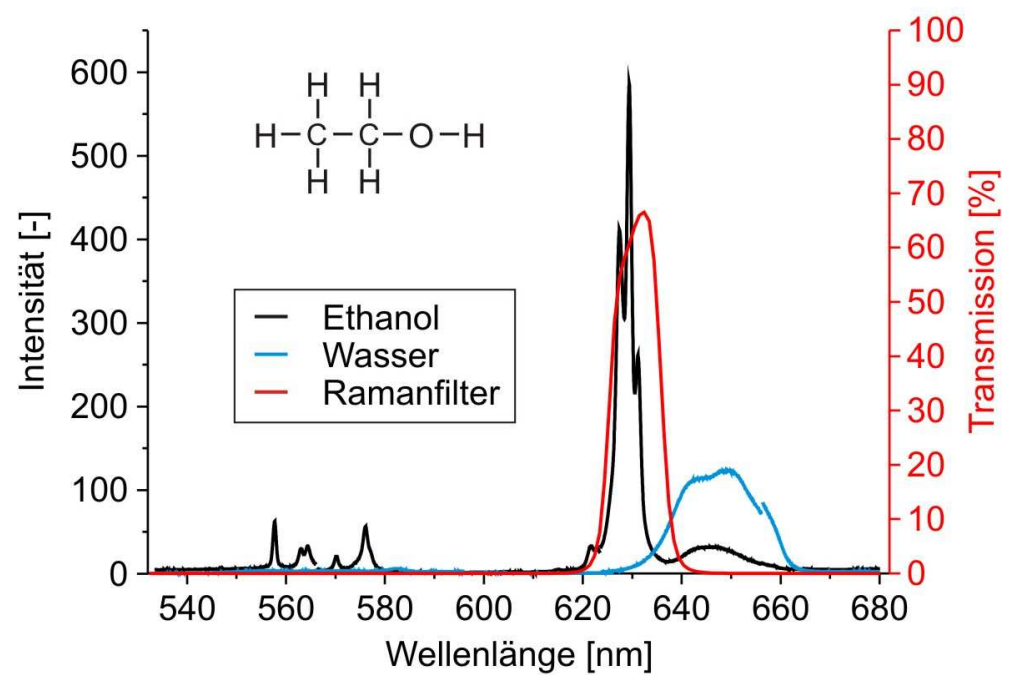

Abb. 5.6.: Ramanspektren der Versuchsfluide Wasser und Ethanol bei Anregung mit 532 nm und Transmissionsspektrum des Ramanfilters

\subsubsection{Mischung Ethanol-Wasser}

Die Mischung von Ethanol und Wasser ist in vielerlei Hinsicht ideal für das Ramanmessverfahren. Die meisten Entwicklungsschritte wurden unter Verwendung dieser Flüssigkeiten durchgeführt. Neben den bereits erwähnten Vorteilen durch die Ungefährlichkeit der Stoffe und ihrer guten Verfügbarkeit sind aus Sicht des zu verwendenden Filters vor allem die spektralen Eigenschaften interessant. Ethanol ist eine organische Flüssigkeit und besitzt daher mehrere CH-Bindungen. Im Ramanspektrum führt dies zu einer besonders intensiven Streulichtbande im Bereich von etwa $3000 \mathrm{~cm}^{-1}$, diese befindet sich bei Anregung mit Laserlicht bei $532 \mathrm{~nm}$ bei etwa $630 \mathrm{~nm}$. Diese Bande entsteht durch Anregung der CH-Streckschwingungen des Moleküls. Wasser ist ein idealer Mischpartner, da es im Gegensatz zu allen organischen Flüssigkeiten keine CH-Bindungen enthält und im Bereich der entsprechenden Bande kein eigenes Ramanlicht aussendet. Die Ramanspektren von Ethanol und Wasser, sowie das Transmissionspektrum des verwendeten Ramanfilters (633FS10-25, LOT Oriel) sind in Abbildung $5.6 \mathrm{zu}$ finden. Um zu verdeutlichen wie schmalbandig die verwendeten Filter sein müssen, wurden in diesem Fall ausnahmsweise keine Wellenzahlen auf der x-Achse aufgetragen, sondern es wurde die intuitiver verständliche Wellenlänge verwendet. Die Ramanspektren wurden in mehreren Teilen mit einem Spektrometer aufgenommen und später zusammengesetzt. Dieses Vorgehen war notwendig, da zur Detektion eine Kamera eingesetzt wurde und die spektral hochauflösenden Gitter des Spektrometers pro Aufnahme nur einen begrenzten Spektralbereich umfassen. Das Transmissionsspektrum des Interferenzfilters wurde mit einem zweiten Spektrometer direkt aufgenommen. Er umfasst sehr genau die CH-Bande, wie sie bei einer Anregung mit $532 \mathrm{~nm}$ Laserlicht auftritt. Das Ramanspektrum von Wasser 


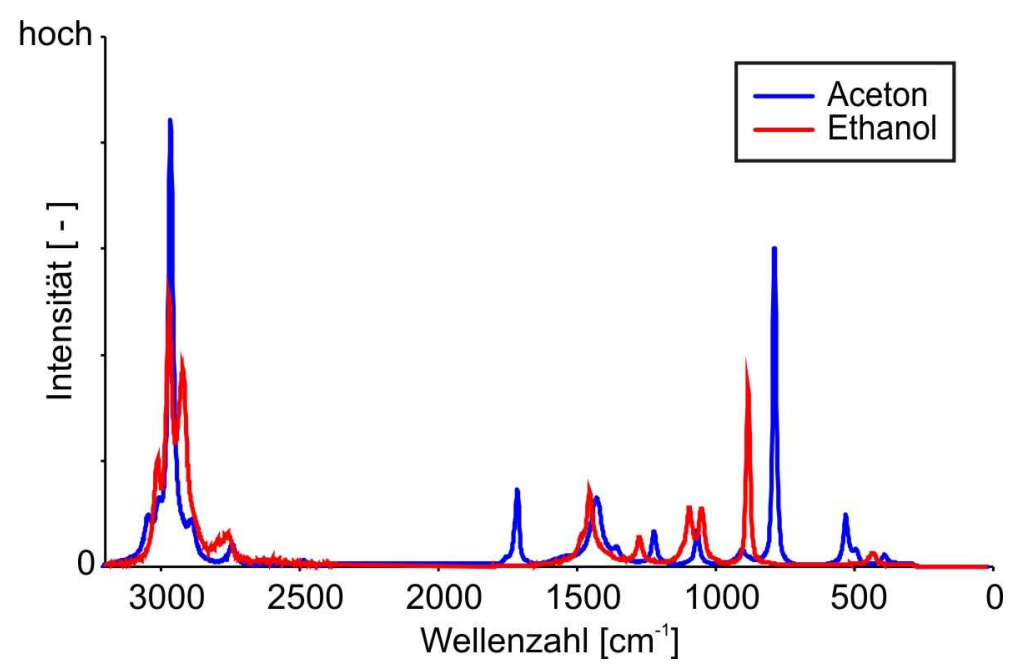

Abb. 5.7.: Ramanspektren von Aceton und Ethanol

überlappt kaum mit dem Transmissionsbereich des Filters, daher kann mit der Kamera selektiv das Ramamstreulicht von Ethanol aufgezeichnet werden. Für quantitative Messungen der Konzentration ist allerdings eine Korrektur der Werte aufgrund der zwar nur kleinen, jedoch nicht zu vernachlässigenden Überlappung des Filtertransmissionsbereiches mit der OH-Ramanbande von Wasser notwendig. In den meisten Fällen lassen sich eingeringfügige Überlappungen auch bei optimal gewähltem Filter nicht vollständig ausschließen. Auf die Anpassung bzw. Korrekturmöglichkeiten wird später genauer eingegangen.

\subsubsection{Mischung Ethanol-Aceton}

Die Mischung von Ethanol und Wasser sorgt für optimale Bedingungen, um den lichtschwachen Ramanprozess zu nutzen. Besonders realistisch ist dieses Szenario jedoch nicht. Ein großer Teil der Flüssigkeiten, die in Mikromischern vermischt werden sollen, ist organisch. Da alle organischen Stoffe CH-Bindungen enthalten, sind ihre Ramanspektren nicht anhand der zugehörigen Ramanbande unterscheidbar. Zur Unterscheidung zweier organischer Flüssigkeiten muss also auf alternative Banden ausgewichen werden, durch die diese Unterscheidung möglich ist. Die Anforderungen an den Ramanfilter steigen bei zwei ähnlichen organischen Stoffen deutlich. Bei der Mischung mit Wasser ist die Suche nach einem Filter einfach, da das Ramanspektrum von Wasser nur eine einzige Bande besitzt. Komplexere Spektren ähnlicher organischer Stoffe besitzen dagegen viele nah beieinanderliegende Banden und der Filter darf nur Licht eines einzelnen Stoffes selektieren. Die Messung wird zusätzlich erschwert, da alternative Banden für gewöhnlich deutlich lichtschwächer als die fundamentale $\mathrm{CH}$-Bande sind.

Im Folgenden wird ein Filter gesucht, der die Untersuchung der Mischung von Ethanol und Aceton ermöglicht. Die Ramanspektren der beiden Flüssigkeiten sind sich sehr ähn- 


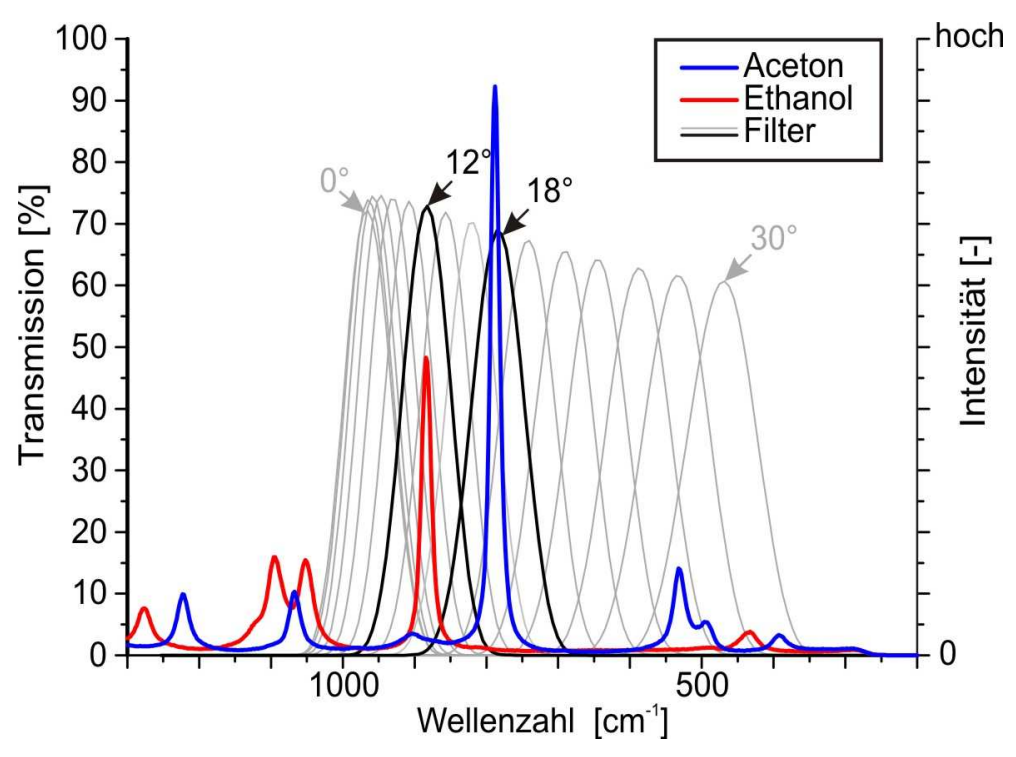

Abb. 5.8.: Ausschnitt der Ramanspektren von Aceton und Ethanol bei Anregung mit 532 nm, sowie Transmissionskurven des Ramanfilters unter verschiedenen Winkeln

lich, wie in Abbildung 5.7 deutlich wird. Da beide Moleküle CH-Bindungen enthalten, ist auch die starke Ramanbande bei $3000 \mathrm{~cm}^{-1}$ in beiden Fällen vorhanden. Durch die abweichende Struktur der Moleküle sind die Banden leicht unterschiedlich ausgeprägt. Trotzdem liegen sie spektral übereinander, was eine Identifizierung eines der Moleküle anhand dieser Bande unmöglich macht. Bei $1700 \mathrm{~cm}^{-1}$ liegt eine einzelne Bande des Aceton, die beim Ethanol nicht vorkommt. Diese ist wegen der geringen Intensität aber nur bedingt geeignet. Der interessanteste Bereich liegt zwischen 700 und $950 \mathrm{~cm}^{-1}$, da die relativ starke Bande an dieser Position in beiden Fällen ausreichend verschoben vorliegt. Es sollte mit einem passenden Filter also möglich sein, die beiden Stoffe zu trennen. Ein Filter zur Messung der Aceton-Verteilung darf maximal den Bereich von 500 bis $840 \mathrm{~cm}^{-1}$ abdecken, da dort nur Ramanstreulicht von Aceton auftritt. Bei einer Anregung mit einer Wellenlänge von $532 \mathrm{~nm}$ wäre dieser Bereich etwa $10 \mathrm{~nm}$ groß.

Eine aufwändige Suche nach dem passenden Filter führte zum Laserline-Filter LL561-25 aus der Maxline-Serie der Firma Semrock. Dieser Filter hat ein sehr schmales Transmissionsspektrum mit einer Halbwertsbreite von ca. $3 \mathrm{~nm}$, welches aber wenige Nanometer neben dem interessanten Spektralbereich liegt.

Durch eine Kippung des Filters gegen die optische Achse konnte der Transmissionsbereich erfolgreich in den relevanten Spektralbereich verschoben werden. In Abbildung 5.8 ist die Region der beiden Maxima der Ramanspektren von Ethanol und Aceton vergrößert dargestellt. Die Transmissionsspektren des Filters unter verschiedenen Winkeln sind in grau, bzw. schwarz eingezeichnet. Die betrachteten Winkeleinstellungen bewegen sich von der Ursprungsposition $\left(0^{\circ}\right)$ bis zu $30^{\circ}$ Verkippung. Bei etwa $12^{\circ}$ wird das EthanolMaximum optimal getroffen, bei $18^{\circ}$ das von Aceton. In beiden Fällen wird kaum Licht 


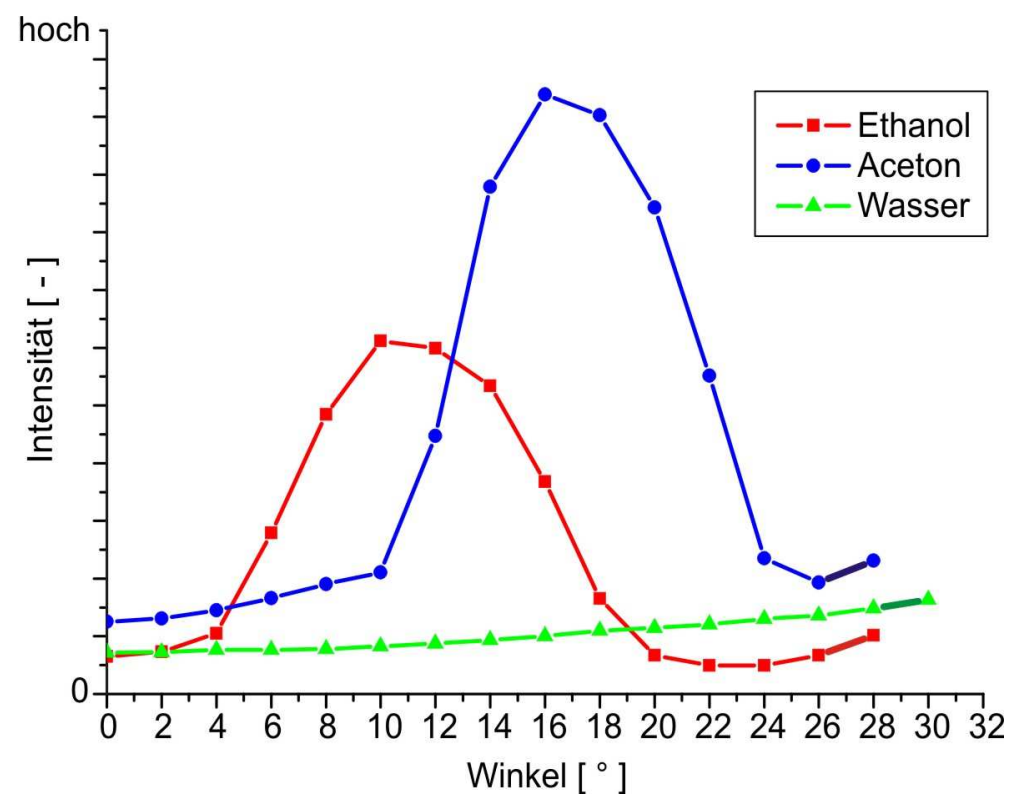

Abb. 5.9.: Intensitätsverlauf bei der Vermessung von Ethanol, Aceton und Wasser mit dem Filter LL561-25 unter verschiedenen Winkeln

des jeweils anderen Mischungspartners transmittiert. Die Intensität dieses unerwünschten Lichts kann durch eine weiter optimierte Winkeleinstellung nahezu vollständig reduziert werden.

In einem einfachen Versuch wurden erste Messungen mit den reinen Fluiden Ethanol, Aceton und Wasser durchgeführt. Der Filter wurde dabei bei jedem Fluid unter verschiedenen Winkeln eingebaut. Es war bei diesen Messungen notwendig 100 Laserschüsse aufzusummieren, da die Intensitäten bei den genutzten Ramanbanden sehr gering sind und das System zum Zeitpunkt dieser Messungen noch nicht vollständig intensitätsoptimiert war. Die Aufnahmen wurden zum Ausgleich von Inhomogenitäten des Laserstrahlprofils zusätzlich örtlich gemittelt über einen Bereich (etwa ein Viertel der gesamten Bildgröße) in der Bildmitte. Auf diese Weise ergibt sich pro Fluid und Filterwinkel ein Intensitätswert. In Abbildung 5.9 sind die Ergebnisse dieser Messungen aufgetragen. Der Verlauf der beiden Intensitätsmaxima in den Ramanspektren von Ethanol und Aceton wird sehr gut nachgezeichnet. Aus der Messung lässt sich nun sehr präzise die optimale Winkeleinstellung des Ramanstreulichtfilters und der zu erwartende Signalbeitrag der jeweils anderen Gemischkomponente ablesen. Zum Vergleich ist auch die, bei den verschiedenen Winkelstellungen zu erwartende, Ramanstreulichtintensität von purem Wasser angegeben. Da Wasser jedoch nur eine einzige Emissionsbande (OH-Schwingung) bei $3300 \mathrm{~cm}^{-1}$ aufweist, sollte die detektierte Ramanstreulichtintensität im betrachteten Spektralbereich Null sein. Die Intensität, die trotzdem in diesem Spektralbereich detektiert wird, liefert daher ein Maß für die Höhe des Untergrundes, der sich im wesentlichen aus Laserstreulicht und thermischem Kamerarauschen zusammensetzt. Man erkennt einen leichten In- 
tensitätsanstieg zu großen Winkeln. Hier ist zu beachten, dass das Licht den Filter nicht als paralleles Strahlenbündel durchquert, sondern verschiedene Winkel auftreten, da eine Abbildung durchgeführt wird. Der Winkel unter dem ein Lichtstrahl tatsächlich auf den Filter trifft, kann sich also von demjenigen unterscheiden, der durch die Verkippung des Filters eingestellt wurde. Damit kann auch Licht den Filter passieren, das unter dem eingestellten Verkippungswinkel nicht transmittiert würde, wenn alle Lichtstrahlen parallel zur optischen Achse verliefen. Daher kann auch ein geringer Anteil des spektral nicht verschobenen Laserstreulichts aus dem Messvolumen den Filter durchdringen und ein Untergrundsignal erzeugen. Eine Kippung des Filters verschiebt den transmittierten Spektralbereich immer weiter in Richtung der Anregungswellenlänge, sodass mehr und mehr Anregungslicht den Filter passiert.

\subsubsection{Beliebige Mischungen}

Ein großes Problem bei der Auswahl eines Ramanfilters ist die fehlende Variabilität in Hinsicht auf den transmittierten Wellenlängenbereich. Wie gezeigt wurde, ist es zwar möglich, das Transmissionspektrum kommerziell erhältlicher Interferenzfilter durch Kippung zu verschieben, die dadurch erzielbare spektrale Verschiebung bewegt sich aber nur im Bereich weniger Nanometer. Außerdem kann die Abbildung durch die Verdrehung des Filters und die unterschiedlichen Einfallsrichtungen des Ramanstreulichts verfälscht werden. Eine schnelle Umschaltung des Messsystems zur Detektion anderer interessierender Substanzen ist daher sehr aufwändig und in einigen Fällen durch Beschränkungen bei der Verfügbarkeit kommerzieller Filter sogar unmöglich. Deutlich bequemer ist die Verwendung eines akusto-optisch einstellbaren Filters (AOTF, von acousto-optic tunable filter), der prinzipiell eine stufenlose Wahl des Transmissionsbereiches ermöglicht. Bei einem AOTF werden Kristallschwingungen genutzt, um einen bestimmten Transmissionsbereich einzustellen. Der Kristall wird dazu durch akustische Anregung in Schwingung versetzt. Je nach Schwingungsfrequenz entsteht im Kristall eine stehende Welle, die den Brechungsindex des Kristalls in einem regelmäßigen Muster verändert. Dies führt zur Ausbildung eines Beugungsgitters. Licht, das unter einem Winkel einfällt, bei dem die Braggbedingung erfüllt ist, wird abgelenkt. Nur dieses Licht der ersten Beugungsordnung wird transmittiert. Das Transmissionsspektrum kann auf diese Weise an eine definierte spektrale Position gefahren werden. Seit einiger Zeit sind AOTFs verfügbar, mit denen großflächigere Abbildungen durchgeführt werden können. Damit eignen sie sich insbesondere für das hier beschriebene Verfahren. Eine aufwändige Suche und Optimierung eines passenden Ramanfilters entfällt, da der AOTF direkt auf die gewünschte Wellenlänge eingestellt werden kann. Bei ersten Tests mit dem vorhandenen AOTF (Model CVA200-0.45-0.7-L, Fa Brimrose) stellte sich heraus, dass die Intensitäten bei der Detektion schmaler Ramanbanden zu gering sind um sinnvolle Messungen durchführen zu können. Das AOTF wurde daher zunächst nicht weiter verwendet und bleibt hier aufgrund seiner ansonsten optimalen Eigenschaften für eine zukünftige Verwendung erwähnt. 


\subsection{Quantitative Kalibration}

Nachdem die Messung der Ramanintensitäten durchgeführt wurde, müssen die Intensitätswerte auf Konzentrationswerte umgerechnet werden. Dazu ist eine Kalibration der Intensitätswerte notwendig. Eine Möglichkeit, eine derartige Kalibration durchzuführen wäre, definierte Konzentrationen der beiden verwendeten Flüssigkeiten $F_{1}$ und $F_{2}$ in der Mischung einzustellen und die resultierenden Ramanstreulichtintensitäten eines der beiden Mischpartner aufzuzeichnen. Damit wäre eine direkte Zuordnung zwischen gemessenen Intensitäten und Konzentration der streuenden Substanz realisiert. Da die Ramanstreulichtintensität direkt proportional zur Anzahl der am Ramanprozess beteiligten Moleküle ist, kann der Proportionalitätsfaktor einfach bestimmt werden. Es ist daher ausreichend, die maximale Intensität zu bestimmen, die bei Anregung des Reinstoffes auftritt. Alle weiteren Konzentrationswerte ergeben sich automatisch aus der angenommenen linearen Abhängigkeit der Signalintensitäten von der Anzahl der streuenden Moleküle im Messvolumen. Die registrierten Streulichtintensitäten $\mathrm{I}_{F 1}$ sind nach Gleichung 4.10 direkt proportional zur Teilchenzahl $\mathrm{N}_{F 1}$, daher ergibt sich die Konzentration $\mathrm{C}_{F 1}^{I}$ der Flüssigkeit $\mathrm{F}_{1}$ aus folgender Definition:

$$
C_{F 1}^{I}:=\frac{N_{F 1}}{N_{F 1}^{r e i n}}=\frac{I_{F 1}}{I_{F 1}^{\text {rein }}}
$$

Dabei liegt die Konzentration $\mathrm{C}_{F 1}^{I}$ zwischen Null und Eins, die Werte $\mathrm{I}_{F 1}^{\text {rein }}$ und $\mathrm{N}_{F 1}^{\text {rein }}$ stellen die zur Kalibration genutzte Ramanintensität des Reinstoffes, bzw. die zugehörige Teilchenzahl dar. Die Intensität $\mathrm{I}_{F 1}^{r e i n}$ wird hier auch als Weißwert der Flüssigkeit $\mathrm{F}_{1}$ bezeichnet. Bisher ist die Bezeichnung „Konzentration“ nur über die Messgröße der aufgezeichneten Sreulichtintensität definiert worden. Bei der Definition aus Gleichung 5.1 spielt es keine Rolle über welche Zustandsgröße die Konzentration definiert wird, da die zu berechnenden Verhältnisse immer denselben Wert ergeben. Für Intensitäten und Teilchenzahlen ist die Äquivalenz aufgrund des Intensitätszusammenhangs (Gleichung 4.10) gegeben. Da die Zustandsgrößen Teilchenzahl, Masse und Volumen jeweils über eine Proportionalitätskonstante (Dichte, Molekülmasse) zusammenhängen, die eine Stoffeigenschaft ausdrückt, sind die Verhältnisse auch für Volumina und Massen ebenfalls gleich denen der Teilchenzahl. Die Stoffeigenschaften können einfach gekürzt werden, da nur eine Molekülsorte betrachtet wird. Es gilt

$$
C_{F 1}^{I}=\frac{N_{F 1}}{N_{F 1}^{r e i n}}=\frac{V_{F 1}}{V_{F 1}^{r e i n}}=\frac{m_{F 1}}{m_{F 1}^{r e i n}} .
$$

Die Definition der Konzentration aus Gleichung 5.1 unterscheidet sich von der verbreiteten Definition der Konzentration. Eine Konzentration gibt zwar immer ein Stoffmengenverhältnis an, so wie es bei der angegebenen Definition gegeben ist, zur Bestimmung eines Konzentrationswertes wird die Menge einer Komponente aber üblicherweise auf die gesamte Mischung, also beide Mischpartner bezogen. Gebräuchlich sind vor allem die folgenden Definitionen für Konzentrationen bei einer binären Mischung der Flüssigkeiten 
$F_{1}$ und $F_{2}$ :

Teilchenzahlkonzentration: $\quad C_{F 1}^{N}:=\frac{N_{F 1}}{N_{F 1}+N_{F 2}}$

Massenkonzentration:

$$
C_{F 1}^{m}:=\frac{m_{F 1}}{m_{F 1}+m_{F 2}}
$$

Volumenkonzentration:

$$
C_{F 1}^{V}:=\frac{V_{F 1}}{V_{F 1}+V_{F 2}}
$$

Die Zustandsgrößen Teilchenzahl, Masse und Volumen könnten bei der Definition einer Konzentration auch vermischt werden, wie z.B. bei der Angabe der Molarität (Teilchenzahl pro Volumen). Bei den folgenden Überlegungen werden solche Varianten aber außer Acht gelassen. Die unterschiedlichen Definitionen des Konzentrationsbegriffs bei der Intensitätsmessung auf der einen Seite und der vorbereiteten Mischung auf der anderen Seite sorgt bei der Kalibration für eine Besonderheit. Die Konzentrationen aus den Gleichungen 5.3, 5.4 und 5.5 sind im Allgemeinen nicht gleich, eine Übereinstimmung mit den Konzentrationen aus Gleichung 5.2 ist folglich ebenfalls nicht unbedingt gegeben. Wenn die Flüssigkeiten $F_{1}$ und $F_{2}$ gemischt werden, ergibt sich die Massenkonzentration von $\mathrm{F}_{1}$, nach der Definition in Gleichung 5.4, aus $m_{F 1} / m_{F 1}+m_{F 2}$. Im Normalfall wird die Dichte der verwendeten Flüssigkeiten unterschiedlich sein, daher unterscheidet sich die Gesamtmasse des Gemischs von der Masse der reinen Substanz innerhalb eines festen Volumens, es gilt $m_{F 1}^{r e i n} \neq m_{F 1}+m_{F 2}$. Damit unterscheiden sich die Bezugsgrößen der beiden Konzentrationsdefinitionen in Gleichung 5.1 (bzw. Gleichung 5.2 für die Masse) und Gleichung 5.4. Auch die Teilchenzahlen im Messvolumen sind bei unterschiedlichen Molekülsorten bei einer Mischung nicht konstant. Die Konzentrationen, die durch die Verhältnisbildung der Ramanintensitäten berechnet wurden, entsprechen in diesen beiden Fällen also nicht der voreingestellten Konzentration. Die Kalibrationskurven wären in diesen Fällen nichtlinear, daher wären auf jeden Fall mehrere Wertepaare zur Kalibration notwendig. Lediglich beim Volumen ergibt sich eine Übereinstimung, da das Messvolumen durch die Messtechnik fest definiert ist. Die Volumenkonzentration aus der Intensitätsmessung entspricht also derjenigen, die man bei Addition der Einzelvolumina bei einer Mischung erhält, es gilt $V_{F 1}^{r e i n}=V_{F 1}+V_{F 2}$. Im Falle der Volumina entsprechen sich damit die beiden Konzentrationsdefinitionen. Der funktionale Zusammenhang der Kalibration ist folglich linear und es reicht eine einzelne Aufnahme zur Kalibration aus.

Voraussetzung für diese lineare Kalibration der gemessenen Intensitätswerte ist, dass die aufgenommene Intensität vollständig aus dem Ramanstreulicht der untersuchten Flüssigkeit resultiert. Tatsächlich lässt es sich meistens jedoch nicht vermeiden, dass auch Streulichtanteile der zweiten Flüssigkeit mit detektiert werden. Entweder sind die Spektren aufgrund ihrer Komplexität zu stark überlagert oder es lässt sich kein Filter finden, der spektral an der richtigen Stelle liegt und schmalbandig genug ist, um nur das Ramanstreulicht einer einzigen Ramanbande zu transmittieren. Die gemessenen Intensitäten sind in diesem Fall eine Mischung aus dem Ramanstreulicht der untersuchten Flüssigkeit und demjenigen der zweiten Gemischkomponente. Zusätzlich gibt es häufig noch Hintergrundstrahlung, die ebenfalls mit aufgezeichnet wird. Letztere kann durch die Subtraktion eines so genannten „Schwarzwertes“ aus den Messdaten entfernt werden. 
Der Schwarzwert wird vor der eigentlichen Messung ohne Laseranregung bestimmt.

Bei den meisten Mischungsvorgängen werden nur zwei Komponenten $\mathrm{F}_{1}$ und $\mathrm{F}_{2}$ gemischt. Im Fall dieser binären Mischungen ist eine quantitative Messung trotz spektraler Überlagerung der Gemischkomponenten möglich. In Gleichung 4.10 sind bei einem gegebenen experimentellen Aufbau alle angegebenen Parameter außer der Teilchenzahl im Mittel konstant. Die Intensitäten hängen also nur noch von der Anzahl der Moleküle der beiden Flüssigkeiten im Messvolumen ab. Die oben angestellten Überlegungen zur Konzentration einer einzelnen Flüssigkeit gelten weiterhin. Die gesamte gemessene Intensität $\mathrm{I}_{\text {mess }}$ entspricht der Addition der Intensitäten, die bei Anregung der einzelnen Flüssigkeiten registriert würden, da sie sich untereinander nicht beeinflussen. Vorausgesetzt wird dabei, dass die Absorption durch die jeweils andere Flüssigkeit verschwindend gering ist und daher keine Rolle spielt. Die Verhältnisse der Molekülzahlen

$$
C_{F 1}^{I}=\frac{N_{F 1}}{N_{F 1}^{r e i n}}=\frac{I_{F 1}}{I_{F 1}^{r e i n}}
$$

und

$$
C_{F 2}^{I}=\frac{N_{F 2}}{N_{F 2}^{r e i n}}=\frac{I_{F 2}}{I_{F 2}^{r e i n}}
$$

bestimmen gemeinsam die aufgenommene Intensität $I_{m e s s}$. Es gilt

$$
\begin{aligned}
I_{\text {mess }} & =I_{F 1}+I_{F 2} \\
& =C_{F 1}^{I} \cdot I_{F 1}^{r e i n}+C_{F 2}^{I} \cdot I_{F 2}^{r e i n}
\end{aligned}
$$

und

$$
C_{F 1}^{I}+C_{F 2}^{I}=1
$$

daher kann die Konzentration der Flüssigkeit $\mathrm{F}_{1}$ berechnet werden durch

$$
C_{F 1}^{I}=\frac{I_{m e s s}-I_{F 2}^{r e i n}}{I_{F 1}^{r e i n}-I_{F 2}^{r e i n}}
$$

In Gleichung 5.10 tauchen neben der gemessenen Intensität $\mathrm{I}_{\text {mess }}$ auch die Intensitäten der Reinstoffe $\mathrm{I}_{F 1}^{\text {rein }}$ und $\mathrm{I}_{F 2}^{\text {rein }}$ auf. Diese Weißwerte werden vor der Messung für die beiden Flüssigkeiten bestimmt. Die Subtraktion eines zusätzlichen Schwarzwertes ist nicht mehr unbedingt notwendig, wenn dieser in allen Intensitätswerten enthalten ist. Er wird dann durch die Subtraktion von $\mathrm{I}_{F 2}^{\text {rein }}$ automatisch bereinigt.

Bei den bisherigen Überlegungen wurde vorausgesetzt, dass die Stoffeigenschaften sich während der Mischung nicht ändern. Dies ist jedoch nicht immer der Fall. Daher kann es unter bestimmten Bedingungen zu Fehlern kommen. Zwei wichtige Fehlerquellen entstehen durch Dichteänderungen. Die Dichte der meisten Flüssigkeiten ist nicht abhängig vom Umgebungsdruck, sie sind inkompressibel. Die Temperatur dagegen spielt bei vielen Flüssigkeiten eine Rolle. Die temperaturinduzierten Dichteunterschiede sind aber gering, 
da davon ausgegangen werden kann, dass die Temperatur sich während einer Messung kaum ändert. Geringe Temperaturunterschiede können während der Vermischung durch den energetisch geänderten Zustand der Lösung auftreten. Höhere Temperaturschwankungen können bei reaktiven Mischungen auftreten, die hier jedoch nicht untersucht werden. Bei flüssigem Wasser kann die Temperatur definitionsgemäß eine Spanne von $100^{\circ} \mathrm{C}$ einnehmen. In diesem Bereich schwankt die Dichte um maximal etwa $4 \%$. Dieser Fehler reproduziert sich linear in den gemessenen Intensitäten.

Eine weitere Fehlerquelle durch Dichteänderung ist die Volumenkontraktion der beiden Flüssigkeiten bei der Mischung. Eine Kontraktion des insgesamt eingenommenen Volumens kann auftreten, wenn die intermolekularen Kräfte zwischen den beiden gemischten Molekülspezies größer sind, als bei jeder einzelnen Spezies. Dadurch sinkt der mittlere Abstand der Moleküle, also auch das Volumen der Lösung. Bei Alkohol und Wasser beträgt die maximale Kontraktion bei einer Mischung von jeweils gleichen Volumina ca. $3 \%$. Bei einer Lösung von $50 \mathrm{ml}$ Wasser und $50 \mathrm{ml}$ Ethanol ergeben sich insgesamt $97 \mathrm{ml}$ der vollständig gemischten Lösung. Diese Abweichung wird umso kleiner, je mehr sich das Lösungsverhältnis den reinen Flüssigkeiten nähert. Im Fall der maximalen Kontraktion können mehr Moleküle das Beobachtungsvolumen belegen als die Berechnung erwarten lässt und die gemessenen Intensitäten liegen etwas zu hoch. Viele Flüssigkeiten zeigen das Phänomen der Volumenkontraktion, in einigen Fällen kann sogar eine Volumenvergößerung auftreten. Für gewöhnlich haben die Volumenänderungen sehr kleine Werte, bzw. sind nicht messbar. Auch dieser Fehler geht linear in die gemessenen Intensitäten ein.

Beide bisher genannten Fehlerquellen führen zu maximalen Fehlern im Bereich weniger Prozent. Empfindliche Regionen sind in beiden Fällen vor allem scharfe Mischungsgradienten, da hier die größten Temperaturdifferenzen auftreten und die Volumenkontraktion bei vollständiger Mischung maximal ist. Die beiden Effekte führen zu Intensitätsänderungen in entgegengesetzte Richtung. Eine höhere Temperatur führt im Allgemeinen zu niedrigeren Dichten, also zu geringeren Teilchenzahlen im Messvolumen und damit zu geringeren Intensitäten. Die Volumenkontraktion sorgt dafür, dass beide Flüssigkeiten insgesamt weniger Raum einnehmen als zuvor. Damit steigt die Teilchenzahl im Messvolumen und ebenso die Intensität. Insgesamt kann davon ausgegangen werden, dass die genannten Fehlerquellen zu einem sehr geringen Gesamtfehler führen, da sie sich eher aufheben als addieren.

In einem einfachen Versuch soll überprüft werden, ob eine lineare Kalibration durch die vorgeschlagenen Konzentrationsdefinitionen möglich ist. Dazu wird in einer Küvette ein festes Massenverhältnis von Ethanol und Wasser gemischt. Die Mischung ist zum Zeitpunkt der Messung vollständig, so dass in jedem Teilbereich dieselbe Intensität gemessen werden sollte. Die gemessenen Intensitäten werden nach Gleichung 5.10 in Konzentrationen umgerechnet. Zu diesem Zweck wurde vor der Messung der Weißwert der Intensitäten der reinen Stoffe bestimmt. Es wurden bei jeder Konzentration mehrere Messungen gemittelt.

Damit zwischen den voreingestellten Konzentrationen und den gemessenen ein linearer Zusammenhang besteht, müssen bei der Berechnung Volumenkonzentrationen verwen- 


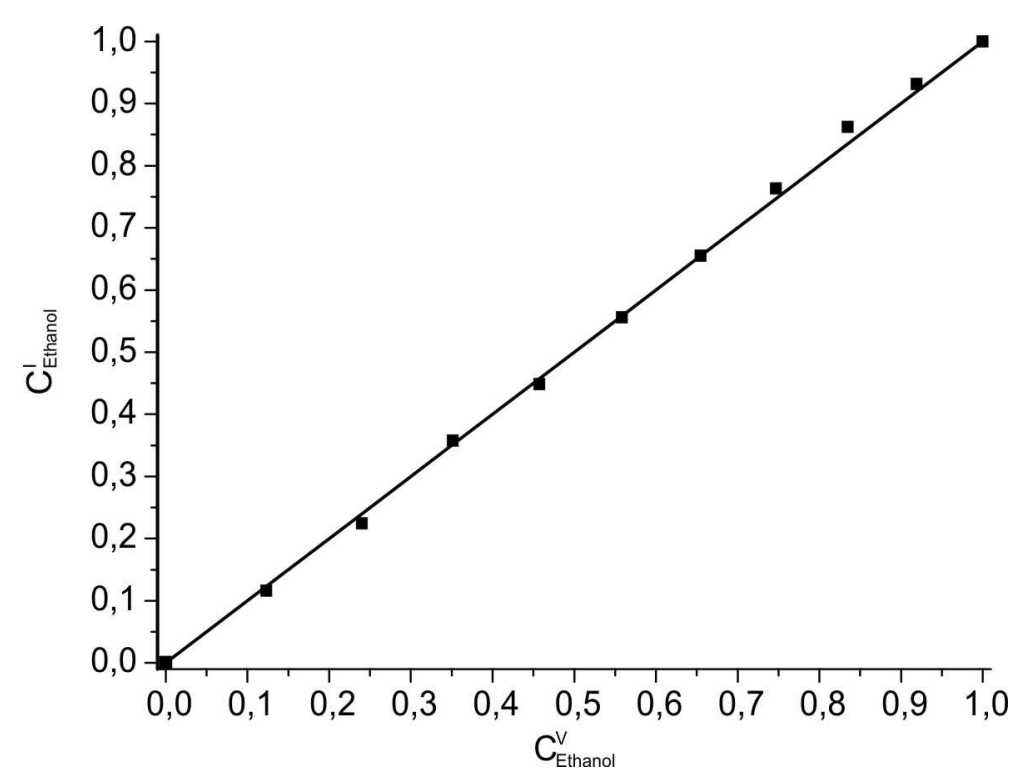

Abb. 5.10.: Vergleich gemessener mit voreingestellten Konzentrationen

det werden. Vorgegeben wurden jedoch die Massenkonzentrationen, daher müssen diese zunächst umgerechnet werden. Abbildung 5.10 zeigt das Ergebnis des Vergleichs. Auf der x-Achse ist die voreingestellte Konzentration $\mathrm{C}_{\text {Ethanol }}^{V}$ und auf der y-Achse die gemessenen Konzentration $\mathrm{C}_{\text {Ethanol }}^{I}$ angegeben. Dabei ist

$$
C_{\text {Ethanol }}^{V}=\frac{m_{\text {Ethanol }}}{m_{\text {Ethanol }}+\frac{\rho_{\text {Ethanol }}}{\rho_{\text {Wasser }}} m_{\text {Wasser }}}
$$

und

$$
C_{\text {Ethanol }}^{I}=\frac{I_{\text {mess }}-I_{\text {Wasser }}^{\text {rein }}}{I_{\text {Ethanol }}^{\text {rein }}-I_{\text {Wasser }}^{\text {rein }}}
$$

beide Größen sind dimensionslos. Bei einer perfekten Übereinstimmung sollten die Werte exakt auf der Winkelhalbierenden liegen, die für eine bessere Bewertung der Messungen ebenfalls eingezeichnet wurde. Die Messwerte weichen im Mittel weniger als $1 \%$ von den tatsächlich eingestellten Konzentrationen ab. Die vorgeschlagene Kalibration aufgrund des linearen Zusammenhangs macht also Sinn und ist sehr genau. Besondere Abweichungen wären aufgrund der genannten Dichteänderungen durch Volumenkontraktion und Temperaturänderung möglich gewesen. Starke Temperaturunterschiede sind während der Messung nicht mehr zu erwarten, da die Mischung bereits deutlich vor der Messung durchgeführt wurde. Lediglich die Volumenkontraktion sollte zu einem geringen Fehler führen, der hier jedoch nicht zu erkennbaren Abweichungen führt. Wie die Messung zeigt, sind mit diesem Verfahren sehr genaue Messungen möglich. 


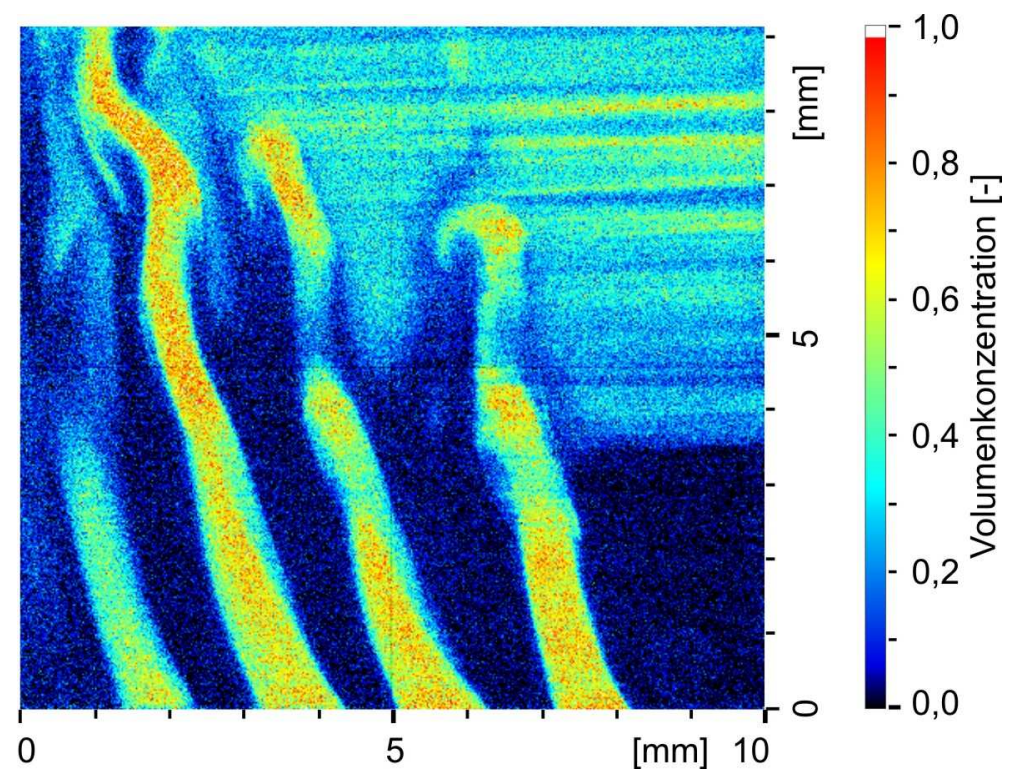

Abb. 5.11.: Zweidimensionale Verteilung der Volumenkonzentration von Ethanol bei Mischung mit Wasser

\subsection{Zweidimensionale Messungen}

Im letzten Abschnitt wurde ein quantitatives Verfahren demonstriert, dass genauso auch für zweidimensionale Messungen genutzt werden kann. Sämtliche Aufnahmetechniken und Berechnungen können, anstatt auf das gemittelte Gesamtbild, auch auf einzelne Bildpunkte angewendet werden. Erst dieser Schritt macht aus der Messprozedur ein zweidimensional quantitatives Messverfahren. Grundlage der quantitativen Messung ist wiederum Gleichung 5.10 bzw. für die verwendeten Flüssigkeiten die konkrete Form in Gleichung 5.12. Für eine bildgebende Messung wird die Berechnung auf jeden einzelnen Bildpunkt angewandt. Es gilt

$$
C_{F 1}^{I}(x, y)=\frac{I_{m e s s}(x, y)-I_{F 2}^{r e i n}(x, y)}{I_{F 1}^{r e i n}(x, y)-I_{F 2}^{r e i n}(x, y)},
$$

mit den beiden Bildkoordinaten x und y. Vor der eigentlichen Messung werden die beiden Weißbilder für Ethanol und Wasser aufgenommen. In beiden Fällen werden jeweils 100 Aufnahmen gemittelt, um ein glattes rauschfreies Bild zu erhalten. Danach können die eigentlichen Messungen durchgeführt und ausgewertet werden. Da nacheinander zwei Weißbilder aufgenommen und die Messung durchgeführt werden, muss darauf geachtet werden, dass alle Aufnahmen unter denselben Versuchbedingungen gemacht werden. Der Bildausschnitt darf sich während der Aufnahmen nicht verändern. Abbildung 5.11 zeigt ein Beispiel einer Messung, bei der die Konzentration des Ethanol auf die beschriebene Art aus den Intensitätsverteilungen der Aufnahmen berechnet wurde. Die Ortsauflösung des Verfahrens wird bestimmt durch die Fläche, die auf einen Bildpunkt des Kamerachips 


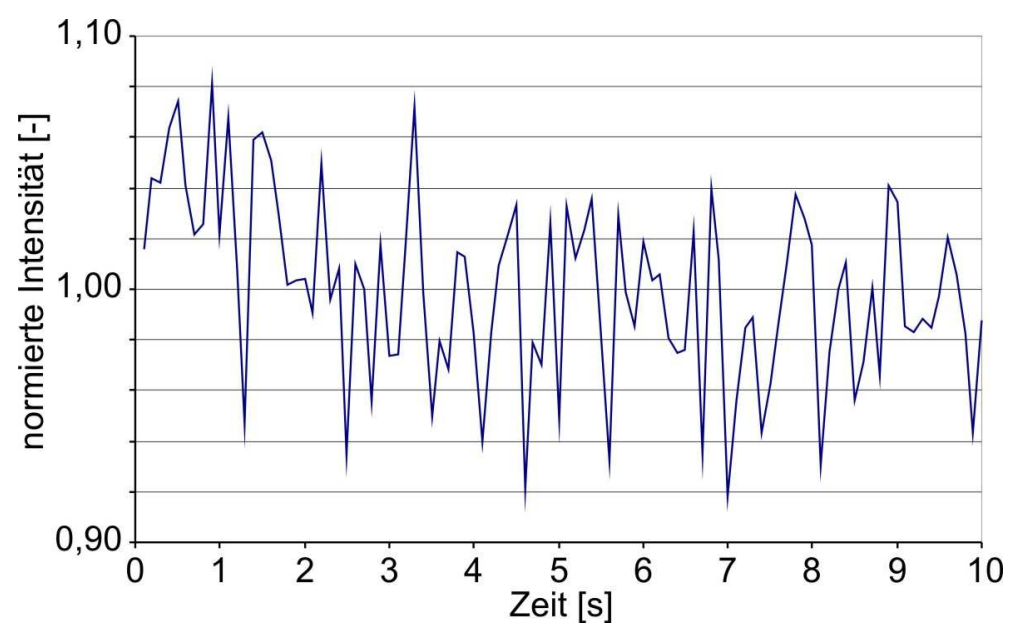

Abb. 5.12.: Intensitätsschwankungen des Lasers, normiert auf die mittlere Intensität

abgebildet wird, sie beträgt bei allen vorgestellten Messungen etwa $20 \times 20 \mu \mathrm{m}$. Die laterale Auflösung hängt von der Dicke des Lichtbandes ab, sie beträgt etwa $500 \mu \mathrm{m}$. Die Intensität, die ein Bildpunkt detektiert, wird durch das zugehörige Volumen, also Fläche mal Lichtbanddicke, bestimmt.

Auffällig sind in dieser Aufnahme die horizontalen Linien im rechten oberen Bildbereich, die aus Sicht der Strömung und Vermischung keinen Sinn machen. Hierbei handelt es sich um ein einfach zu erklärendes optisches Phänomen. Die beiden verwendeten Flüssigkeiten haben einen unterschiedlichen Brechungsindex. Besonders in Bereichen starker Konzentrationsgradienten existieren daher auch hohe Brechungsindexgradienten. An diesen Stellen wird der Laserstrahl abgelenkt, wodurch im weiteren Verlauf des Laserstrahls erhöhte, bzw. verringerte Laserintensitäten auftreten. Da diese Laserstrahlinhomogenitäten bei der Aufnahme der Weißbilder aufgrund fehlender Brechungsindexgradienten nicht auftreten, können sie nicht korrigiert werden und führen bei der Kalibration zu lokal falschen Konzentrationswerten. Die Brechungsindexunterschiede sorgen außerdem für eine schlechtere Abbildung der Messebene auf die Kamera. Vor allem die, aus Sicht der Kamera, weiter hinten liegenden Ebenen im Kanal erscheinen unscharf. Je mehr Brechungsindexgradienten bei der Abbildung durchlaufen werden, desto stärker wird die Unschärfe. Eine Möglichkeit, die auftretenden Fehler zu vermeiden, ist eine Anpassung des Brechungsindexes der beiden Flüssigkeiten.

Bei Betrachtung einer längeren Serie von Aufnahmen einer einzelnen homogenen Flüsigkeit fällt auf, dass die aufgenommenen Intensitäten von Bild zu Bild deutlich schwanken. Diese globalen Helligkeitsschwankungen werden durch Intensitätsschwankungen des verwendeten Nd:YAG-Lasers hervorgerufen. Solange diese Schwankungen nicht simultan mit der Detektion der Ramanstreulichtverteilung erfasst werden, ist eine Quantifizierung der Signalintensitäten nur im Rahmen der Schwankungsbreite der Laserintensität möglich. Abbildung 5.12 zeigt die während einer Messung auftretenden Laserschwan- 


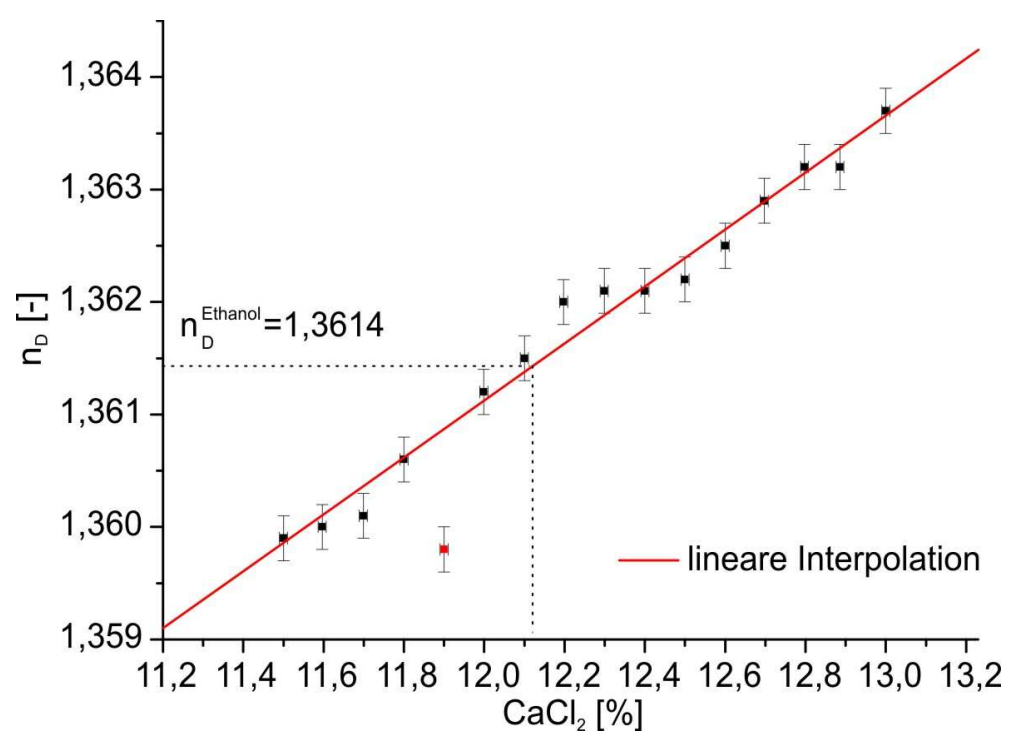

Abb. 5.13.: Brechungsindexanpassung an Ethanol durch $\mathrm{CaCl}_{2}$ in Wasser

kungen. Für diese Aufzeichnung wurde reines Ethanol ohne Strömung aufgenommen, so dass die Intensitätsänderungen ausschließlich vom anregenden Laser erzeugt werden. Die Intensität wurde jeweils über das gesamte homogen ausgeleuchtete Bild gemittelt. Die Intensitätswerte wurden zuletzt auf ihren zeitlichen Mittelwert normiert. Man kann dadurch die maximalen Intensitätsabweichungen vom Mittelwert angeben. In diesem Fall liegen sie bei etwa $8 \%$.

Im Folgenden werden Maßnahmen beschrieben, die eine Vermeidung bzw. Reduzierung der dargestellten Probleme bei der Quantifizierung der Ramanstreulichtintensitäten ermöglichen.

\subsubsection{Brechungsindexanpassung}

Die unterschiedlichen Brechungsindizes der beiden verwendeten Substanzen Ethanol und Wasser führen zu Strahlablenkungen und Fokussierungseffekten. Abhilfe kann geschaffen werden, indem die Brechungsindizes der Flüssigkeiten durch einen Zusatzstoff angeglichen werden. Die Anpassung wird durch einen Zusatzstoff erreicht, der den Brechungsindex einer der beiden Flüssigkeiten verändert. Es spielt dabei keine Rolle, ob der Brechungsindex von Wasser auf den von Ethanol angepasst wird oder umgekehrt. Der Zusatzstoff darf kein störendes zusätzliches Raman- oder Fluoreszenzlicht emittieren.

Ein möglicher Zusatzstoff ist Calciumchlorid $\left(\mathrm{CaCl}_{2}\right)$, das in Wasser gelöst wird. Der Brechungsindex von Wasser $n_{D}^{\text {Wasser }}=1,33$ (bei $20^{\circ} \mathrm{C}$ und $589 \mathrm{~nm}$ ) kann damit an denjenigen von Ethanol $n_{D}^{\text {Ethanol }}=1,3614$ angepasst werden. Aus Abbildung 5.13 kann die ideale Massenkonzentration von $\mathrm{CaCl}_{2}$ in Wasser abgelesen werden. Mit einer Feinwaage wurden definierte Mengen $\mathrm{CaCl}_{2}$ abgewogen und anschließend in Wasser gelöst, 


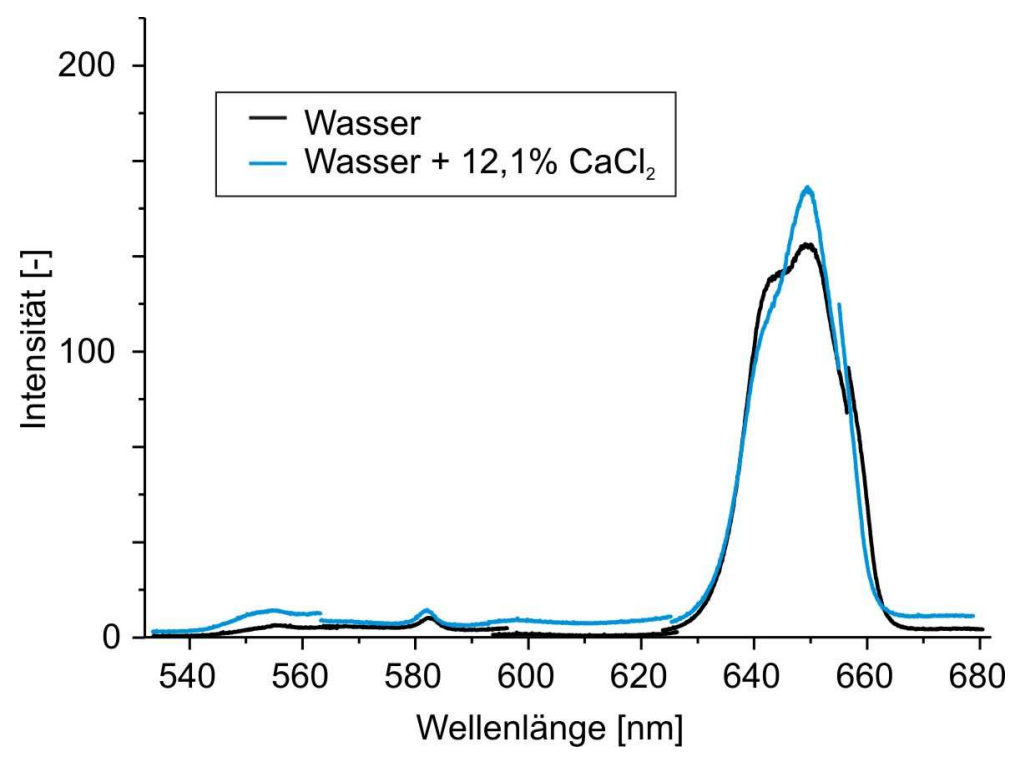

Abb. 5.14.: Vergleich der Spektren von reinem Wasser und Wasser mit 12,1 \% $\mathrm{CaCl}_{2}$

um unterschiedliche Konzentrationen der Lösung einzustellen. Der Brechungsindex der jeweiligen Lösung wurde mit einem Abbe-Refraktometer bestimmt und gegen die Konzentration aufgetragen. Nach Anpassung einer linearen Ausgleichsgeraden an die Daten der Messungen (rote Linie) kann die ideale Konzentration von ca. 12,1\% abgelesen werden (gestrichelte Linien). $\mathrm{CaCl}_{2}$ löst sich auch in größeren Mengen sehr gut in Wasser. Eine entsprechende Löslichkeit des $\mathrm{CaCl}_{2}$ muss aber in beiden Flüssigkeiten gegeben sein, damit es bei der späteren Mischung nicht wieder ausfällt. Eine Massenkonzentration des $\mathrm{CaCl}_{2}$ von $12,1 \%$ ist problemlos sowohl in Wasser als auch in Ethanol löslich.

Ein Vergleich der Spektren von reinem Wasser und Wasser mit dem gelösten $\mathrm{CaCl}_{2}$ ist in Abbildung $5.14 \mathrm{zu}$ sehen. Die Spektren wurden jeweils aus mehreren teilweise überlappenden Teilspektren zusammengesetzt, was sich insbesondere durch eine Unstetigkeit in der Ramanbande von Wasser bemerkbar macht. Man erkennt deutlich, dass sich beide Spektren ansonsten kaum unterschieden. In Lösung liegt das $\mathrm{CaCl}_{2}$ in dissoziierter Form $\left(\mathrm{Ca}^{2+}\right.$ und $\left.\left(\mathrm{Cl}^{-}\right)_{2}\right)$ vor, sodass keine Molekülschwingungen auftreten können und kein Vibrationsramanspektrum emittiert wird. Das Potential der OH-Bindung in den Wassermolekülen wird durch den Einfluss der $\mathrm{CaCl}_{2}$-Ionen leicht verformt, sodass auch die spektrale Struktur der Vibrationsramanbande von Wasser geringfügig verändert wird. Desweiteren ist $\mathrm{zu}$ erkennen, dass durch die Zugabe von $\mathrm{CaCl}_{2}$ keine störenden Fluoreszenzerscheinungen erzeugt werden. Aus spektraler Sicht ist $\mathrm{CaCl}_{2}$ also ein idealer Zusatzstoff zur Brechungsindexanpassung.

Bei der Lösung von $\mathrm{CaCl}_{2}$ in Wasser wird viel Energie in Form von Wärme frei, daher muss die Lösung noch einige Zeit ruhen, bevor sie im Experiment eingesetzt werden kann. Bei deutlich unterschiedlichen Temperaturen zwischen der $\mathrm{CaCl}_{2}$-Lösung und Ethanol wäre jede Anpassung des Brechungsindex hinfällig, da dieser auch von der Temperatur 


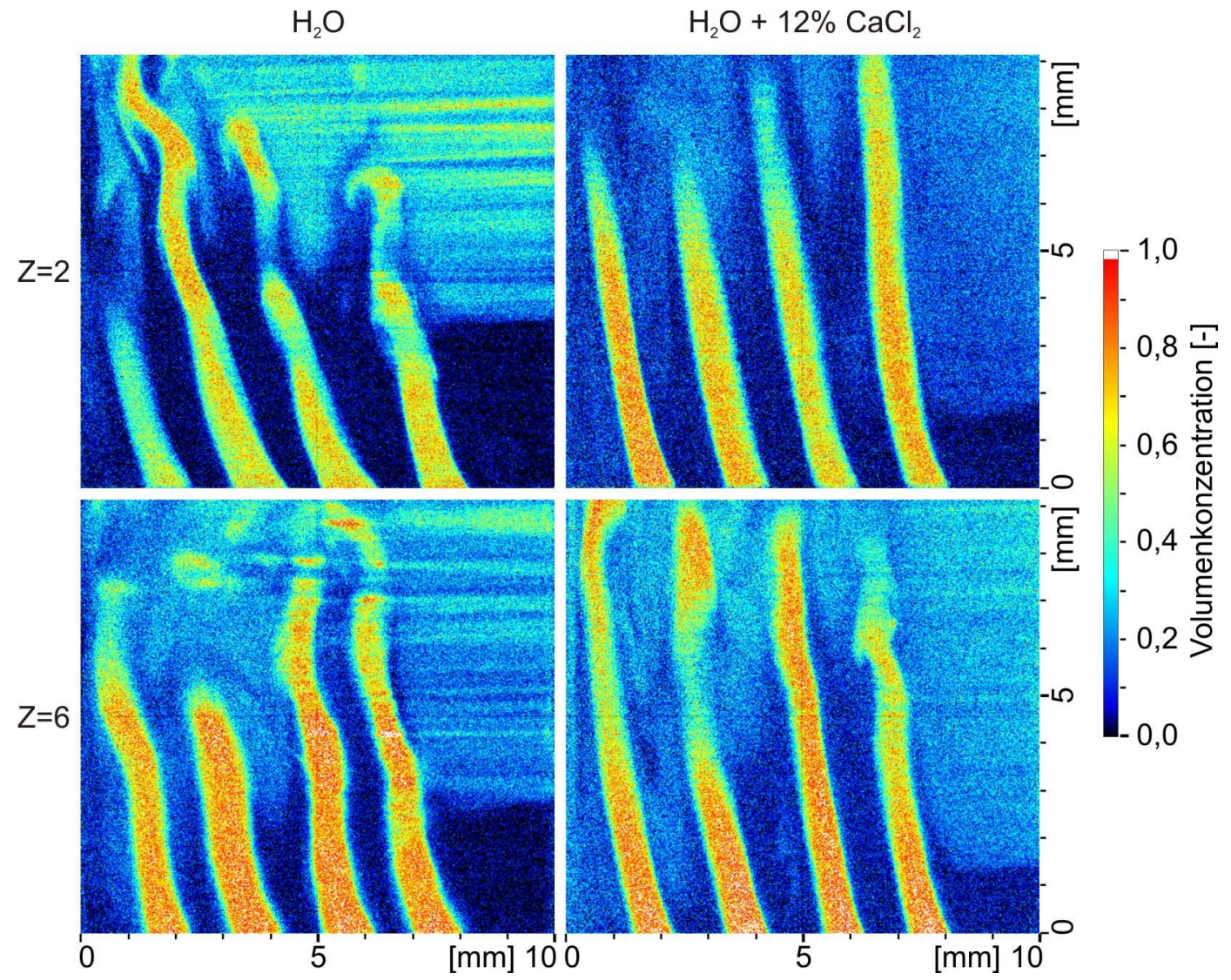

Abb. 5.15.: Ethanolkonzentration in Wasser, linke Spalte: reines demineralisiertes Wasser, rechte Spalte: angepasster Brechungsindex durch Zugabe von $\mathrm{CaCl}_{2}$, obere Zeile: Messung bei $Z=2$, untere Zeile: Messung bei $Z=6$

abhängig ist. Durch die Wartezeit beim Temperaturausgleich muss eine weitere langsame Reaktion berücksichtigt werden. Bei der Lösung von $\mathrm{CaCl}_{2}$ in Wasser fällt durch Reaktion mit dem im Wasser gelösten Kohlendioxid nach einer Weile Kalk aus, was zu einer Trübung der Lösung führt. Zur Unterdrückung dieser Reaktion wird das Wasser vor der Mischung entgast und unter Luftabschluss gehalten. Zur Entgasung wurde die Flüssigkeit eine halbe Stunde auf $90^{\circ} \mathrm{C}$ erhitzt und anschließend 15 Minuten in ein Ultraschallbad gestellt. Der ausgefallene Kalk wird vor der Messung ausgefiltert, so dass keine Störung der Messung durch Streuung des anregenden Laserlichts an den Kalkpartikeln entsteht. Nach der Filtrierung kann ausreichend lange mit der Lösung gearbeitet werden bis sich neuer Kalk bildet.

Die Brechungsindexanpassung sorgt für sichtbar bessere Ergebnisse, wie Abbildung 5.15 zeigt. Bei den beiden linken Aufnahmen wurde reines demineralisiertes Wasser verwendet, bei den rechten wurde der Brechungsindex durch Zugabe von $\mathrm{CaCl}_{2}$ an den von Ethanol angepasst. Bei den Aufnahmen mit Brechungsindexanpassung existieren nahezu keine Fokussierungen mehr. Auch in der Tiefe der Mischstrecke ist die Schärfe 
verbessert. Die Aufnahmen der oberen Zeile wurden weit vorn gemacht, während die der unteren Zeile weiter hinten entstanden. Der Strahlengang der Abbildung muss dabei mehr Regionen mit hohen Brechungsindexgradienten durchdringen und wird unscharf. Ohne Brechungsindexanpassung ist die Schärfe der aufgenommenen Filamente im hinteren Teil der Mischstrecke leicht vermindert, während sie mit Anpassung gleich bleibt.

Bei der Untersuchung instationärer strömungsphysikalischer Vorgänge ist die Betrachtung von Mittelwerten und deren Standardabweichung sehr aussagekräftig. Vor allem die Standardabweichung liefert Informationen über zeitliche Variationen des lokalen Strömungsfeldes. Diese würde besonders durch die von zeitlich instationären Fokussierungseffekten hervorgerufenen Intensitätsschwankungen deutlich verfälscht.

\subsubsection{Ausgleich von Intensitätsschwankungen und Inhomogenitäten}

Intensitätsschwankungen der Laserstrahlung wurden bereits bei der quantitativen Auswertung von Intensitäten als Problem beschrieben. Grundsätzlich können zwei verschiedene Arten von Intensitätsschwankungen auftreten. Globale Schwankungen beziehen sich auf die mittlere Laserenergie eines Laserpulses. Lokale Schwankungen bewirken eine Änderung der Energieverteilung innerhalb des Strahlprofils. In beiden Fällen sorgen die Schwankungen für Abweichungen der Intensitätswerte von denjenigen, die zuvor bei den gemittelten Intensitäten der Weißbilder registriert wurden. Damit treten Fehler bei der Berechnung der Konzentrationswerte auf. Globale Schwankungen der Laserintensität können durch eine Referenzmessung korrigiert werden. Der präsentierte Versuchsaufbau enthält eine homogenisierende Optik, um die Strahlprofiländerungen zu korrigieren und die lokalen Intensitätsschwankungen zumindest teilweise auszugleichen.

Wie bereits in Kapitel 5.4 erläutert wurde, detektiert eine zusätzliche Kamera in einem Referenzstrahlengang die Intensität des Anregungslasers simultan zu der eigentlichen Messung, um globale Schwankungen auszugleichen. Jeder Aufnahme der Messung kann eine Referenzaufnahme zugeordnet werden. Für die Korrektur wird die Intensität jeder Referenzaufnahme räumlich gemittelt. Die Intensitätswerte werden auf den zeitlichen Mittelwert der Serie normiert, wodurch sich für jede Aufnahme ein Faktor ergibt, der die Abweichung vom Mittelwert beschreibt. Jede Aufnahmen der Messung wird durch den zugehörigen Faktor aus der Referenzmessung dividiert, dadurch werden die Intensitäten auf den globalen Mittelwert normiert. Mit einer entsprechenden Makroprogrammierung ist im Anschluss an die Messung eine automatische Auswertung und Korrektur der Schwankungen möglich.

Abbildung 5.16 zeigt den bereits aus Abbildung 5.12 bekannten Verlauf der Laserpulsintensitäten, die im Messarm in reinem Ethanol detektiert wurden. Auch bei diesen Messungen wurden zeitlich synchronisiert die Intensitäten im Referenzarm registriert. Beide Intensitätsverläufe sollten in diesem Fall gleich aussehen, da in beiden Fällen reines Ethanol verwendet wurde. Das beschriebene Korrekturverfahren wurde auf die beiden Datensätze angewendet. Die resultierende Kurve sollte idealerweise im gesamten Verlauf bei eins liegen und keine Schwankungen mehr aufweisen. Die rote Kurve zeigt den korrigierten Verlauf der Intensitäten. Die globalen Unterschiede sind erheblich geringer und die korrigierten Intensitäten bleiben vor allem von Bild zu Bild deutlich konstanter. Nach 


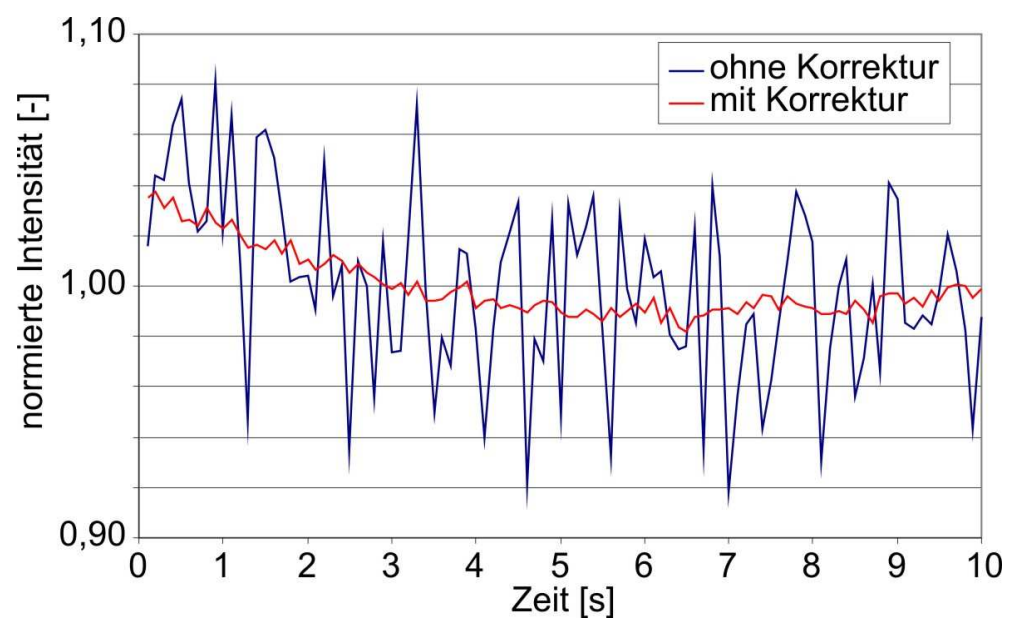

Abb. 5.16.: Korrektur der Intensitätsschwankungen der Laserstrahlung durch Referenzmessungen

der Korrektur ist trotzdem noch ein Intensitätsverlauf zu erkennen, der sich vom idealen Wert unterscheidet. Der Verlauf ergibt sich aller Wahrscheinlichkeit nach durch die Tatsache, dass für die Aufnahmen zwei verschiedene Kameras verwendet wurden, bei denen ein zeitlich gegeneinander schwankender Untergrund thermischen Rauschens durch die nicht synchronisierte Temperaturregelung generiert wird. Diese Vermutung konnte mit einer Testmessung an einer zeitlich konstanten Lichtquelle bestätigt werden. Der Fehler, der sich aus der Verwendung zweier Kameras ergibt, lässt sich nicht ohne weiteres korrigieren, da die Intensitätsabweichungen nicht bei jeder Messung gleich verlaufen. Die einfachste Möglichkeit wäre die Verwendung eines einzigen Kamerachips, indem auf die Chipfläche beide Abbildungen nebeneinander gelegt werden. Aufgrund des relativ hohen Justageaufwands wurde diese Variante jedoch nicht durchgeführt. Auch mit dem nicht korrigierten systematischen Fehler wurden die Intensitätsschwankungen des Anregungslasers nahezu eliminiert. Die maximale Abweichung vom Mittelwert liegt unter 4 \%. Die Schwankungen von Bild zu Bild konnten sogar noch deutlich stärker reduziert werden. Sie liegen nach der Korrektur im Bereich von einem Prozent.

Neben den globalen Schwankungen treten auch Puls zu Puls-Variationen im Strahlprofil auf. Damit diese Schwankungen möglichst keine Auswirkungen auf die gemessenen Konzentrationsverteilungen haben, wird der Laserstrahl gleichzeitig mit der Strahlformung homogenisiert. Zu diesem Zweck wurde von der Firma Suss Microoptics eine Kombination verschiedener Linsenelemente zusammengestellt, die ein sehr dünnes homogenisiertes Laser Lichtband erzeugt. Ein ähnliches System ist in [57] beschrieben. Bei einer optischen Strahlhomogenisierung wird der Ausgangsstrahl in viele kleine Bereiche zerteilt, die durch die Optiken jeweils auf den gleichen Bildbereich abgebildet werden. Man erreicht dadurch eine gleichmäßige Überlagerung aller Strahlbereiche des Ausgangsstrahls. Das Prinzip ist in Abbildung 5.17 skizziert. Der Aufbau besteht aus zwei aufeinander folgenden Zylinderlinsenarrays (Typ C-Q-500 2,5 , Fa. Linos) und einer 

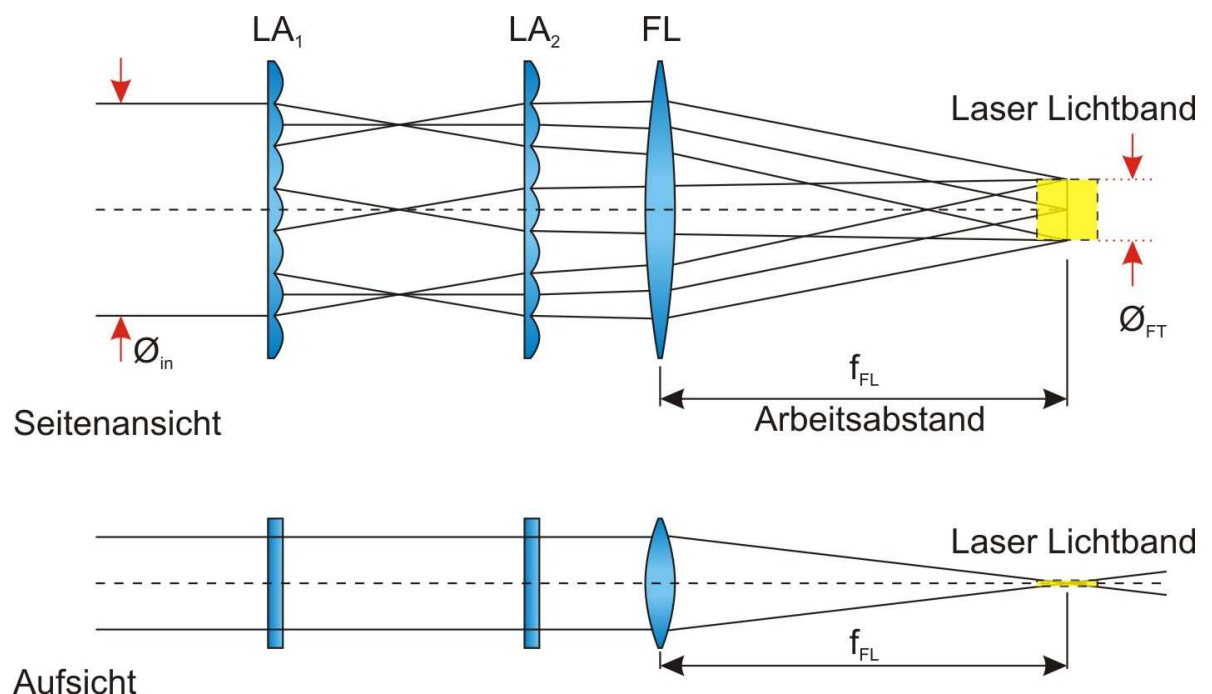

Abb. 5.17.: Strahlhomogenisierung und -formung des Laser Lichtbandes durch Linsenarrays

sphärischen Fourierlinse $(\mathrm{f}=200 \mathrm{~mm})$. Im Prinzip bilden diese Elemente in der Seitenansicht einen abbildenden Homogenisator. Der eingehende Laserstrahl $\varnothing_{\text {in }}$ wird durch ein Linsenarray in möglichst feine Teilstrahlen aufgeteilt. Eine Fourierlinse übernimmt anschließend in Kombination mit einem zweiten Array die Abbildung auf eine definierte Region $\varnothing_{F T}$. In dieser Region werden alle Strahlbereiche vereinigt. Dadurch werden Bereiche geringerer und Bereiche höherer Intensität summiert. Im Beispiel werden die drei eingezeichneten Bereiche des Ausgangsstrahls oben, in der Mitte und unten in derselben Fläche überlagert. Das Ergebnis ist eine homogene Intensitätsverteilung. In der Aufsicht zeigt sich, dass die Linsenarrays in dieser Dimension keinen Effekt haben. Die Strahlformung geschieht allein durch die sphärische Linse, die in der Region der Strahltaille das Laser Lichtband bildet. Die Brennweite der Linse ist mit $\mathrm{f}=200 \mathrm{~mm}$ relativ lang gewählt, um eine möglichst gleich bleibende Dicke des Lichtbandes im Bereich der Taille zu erzeugen. Das Lichtband ist im Bereich der Taille ca. $500 \mu \mathrm{m}$ dick.

Durch die Kombination von Strahlhomogenisierung und Behebung von Intensitätsschwankungen konnten die Fehler bei der Konzentrationsmessung deutlich reduziert werden. Die größte Fehlerquelle stellen die globalen Intensitätsschwankungen des Laserstrahls dar. Diese Schwankungen konnten von maximal $10 \%$ auf etwa $4 \%$ Prozent reduziert werden.

\subsection{Messungen}

Zur Vermessung des verwendeten Mikromischers wurde die Mischstrecke in mehrere Ebenen unterteilt. Abbildung 5.18 zeigt die Messpositionen im Multilaminationsmischer. Die gesamte Mischstrecke ist ca. $40 \mathrm{~mm}$ lang, davon sind ungefähr $35 \mathrm{~mm}$ optisch zugänglich, 

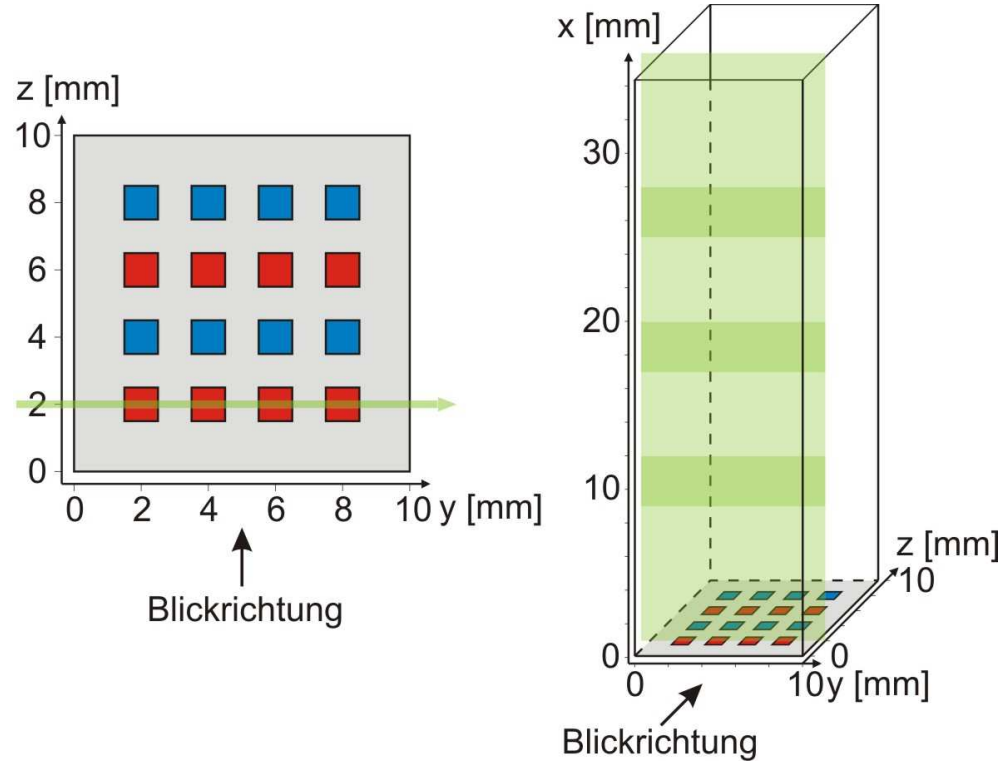

Abb. 5.18.: Messpositionen im Multilaminationsmischer

da der oberste Bereich durch Schrauben und Muttern der Halterung verdeckt wird. Die Querschnittsfläche der Mischstrecke ist genau $10 \times 10 \mathrm{~mm}$ groß. Der Bildbereich der Kamera ist so eingestellt, dass etwas mehr als die Breite der Mischstrecke von $10 \mathrm{~mm}$ erfasst wird, er misst etwa 10,5×10,5 mm. Die Hauptströmungsrichtung verläuft in der Mischstrecke von unten nach oben, daher ist die $\mathrm{x}$-Achse in diese Richtung angeordnet. Die y-Achse verläuft von links nach rechts und die z-Achse von vorn nach hinten. Nach dieser Notation stellen die vom Laser Lichtband aufgespannten Flächen also x-y-Ebenen dar. In z-Richtung wurden insgesamt neun Messpositionen $(\mathrm{z}=1 \mathrm{~mm}, \mathrm{z}=2 \mathrm{~mm}, \ldots, \mathrm{z}=9 \mathrm{~mm}$ ) aufgenommen. Die Ebene $\mathrm{z}=2 \mathrm{~mm}$ ist als Beispiel in der Aufsicht links in Abbildung 5.18 eingezeichnet. Entlang der Küvettenachse wurden vier Positionen vermessen. Die Messpositionen überlappen ca. 2,5 mm. Damit ergibt sich ein Messbereich von $34 \mathrm{~mm}$. Die Mischstrecke wird also nahezu vollständig erfasst. Die vier Positionen sind bei $\mathrm{z}=2 \mathrm{~mm}$ in der dreidimensionalen Zeichnung rechts in Abbildung 5.18 eingezeichnet. Der dunklere Bereich entspricht der Überlappung, die in jeweils zwei Messungen aufgenommen wurde. Insgesamt ergeben sich 36 Messpositionen, um die gesamte Mischstrecke zu erfassen. Der Einfachheit halber werden die Messpositionen im Folgenden in jeder Dimension in Richtung der Achsen durchnummeriert. Die Messposition X1Z2 liegt also beispielsweise bei $\mathrm{z}=2 \mathrm{~mm}$ und umfasst den Bereich $\mathrm{x}=0-10,5 \mathrm{~mm}$ und $\mathrm{y}=0-10 \mathrm{~mm}$. In jeder Messposition wurden 100 Aufnahmen mit einer Aufnahmefrequenz von $2 \mathrm{~Hz}$ registriert. Vor einer Messung wurden die beiden Weißbilder der Reinstoffe Ethanol und Wasser aufgenommen, um die Intensitäten nach der Messung in Konzentrationsverteilungen umrechnen zu können. Die Umrechnung wurde, wie in den Kapiteln 5.6 und 5.7 beschrieben, durchgeführt. Zur Reduktion des Rauschens wurden die Daten anschließend durch eine Intensitätsmittelung 
über jeweils $3 \times 3$ Bildpunkte geglättet.

Das Mischverhalten wurde jeweils bei einer langsamen und einer schnellen Strömungsgeschwindigkeit aufgenommen. Die Strömungsgeschwindigkeiten wurden durch den Hinterdruck des Gases und das Nadelventil reguliert und vor einer Messung passend eingestellt. Ein Absperrventil verschloss bis zur Messung das Reservoir, damit möglichst wenig Flüssigkeit verwendet wurde. Mindestens zehn Sekunden vor Beginn der Messung wurde das Absperrventil geöffnet, damit die Strömung sich stabilisieren konnte. Direkt nach der Messung wurde das Absperrventil wieder verschlossen und anschließend eine neue Messposition einjustiert. Während jeder Messung wurde der Volumenfluss beider Flüssigkeiten aufgezeichnet. Dieser wurde bestimmt durch die Messung der Höhendifferenz der Flüssigkeitspegel und eine Zeitmessung mit einer Stoppuhr. Es wurde davon ausgegangen, dass der Durchfluss zwischen Öffnen und Schließen des Absperrventils näherungsweise konstant ist. Wenn man die einzelnen Messpositionen aneinander reiht, kann es aufgrund unterschiedlicher Durchflusswerte zu einem Versatz der Konzentrationsgradienten kommen. Trotz einer guten Entgasung der Flüssigkeiten vor einer Messung können sich durch Ausgasen ab und zu Blasen bilden. Diese stellen normalerweise kein Problem dar, da sie mit der Strömung nach oben treiben und nur in einzelnen Aufnahmen für sichtbare Fehler sorgen. Vor allem bei der langsameren Strömung werden Blasen aber nicht unbedingt von der Strömung mitgerissen und können damit ein stehendes Strömungshindernis im Zulauf bilden, das zwar in den Aufnahme nicht sofort zu bemerken ist, die Strömung aber dauerhaft verändert. Dadurch kann wiederum ein Versatz zwischen den Strömungsgradienten zweier überlappender Positionen entstehen.

\subsubsection{Durchflussmessung}

Um die Gemischbildung bei unterschiedlichen Strömungsgeschwindigkeiten zu Charakterisieren, wurden flächige Konzentrationsverteilungen für jeweils eine langsame und eine schnelle Strömung vermessen. Zunächst wurden Messungen bei einer langsamen Strömung durchgeführt. Die Strömungsgeschwindigkeit wurde wie beschrieben vor den Messungen eingestellt, indem ein definierter Hinterdruck und eine bestimmte Einstellung der Nadelventile für beide Flüssigkeiten gewählt wurde. Der Durchfluss wurde dann mit einem Absperrventil gestoppt und während der Messungen nur noch durch dieses reguliert. Für beide Flüssigkeiten wurden identische Strömungsgeschwindigkeiten eingestellt. Die Auswertung der mittleren Strömungsgeschwindigkeiten aus den gemessenen Zeiten und Höhenunterschieden ergaben jedoch auch im Mittel aller Messungen Abweichungen. Die ermittelten Durchflusswerte sind in Tabelle 5.1 aufgelistet. In der Tabelle sind die Positionen, an der die Messungen durchgeführt wurden, nach der bereits beschriebenen Nomenklatur angegeben. Die weiteren Spalten listen in zwei Bereichen die gemessenen Strömungsgeschwindigkeiten für die langsame und die schnelle Strömung auf. Es sind für beide Flüssigkeiten jeweils die Durchflusswerte in $\mathrm{ml} / \mathrm{min}$ und die Abweichung vom Mittelwert aller Messungen in Prozent angegeben. Die Mittelwerte sind in der letzten Zeile eingetragen. Die Reihenfolge der Messungen entspricht (mit Ausnahme von X1Z1 und X1Z2 bei der langsamen Strömung) genau dem chronologischen Ablauf der Messungen. Abgegrenzt sind die Übergänge zu einer neuen Schicht in X-Richtung, da die 


\begin{tabular}{|c|c|c|c|c|c|c|c|c|}
\hline \multirow{3}{*}{ Position } & \multicolumn{4}{|c|}{ langsame Strömung } & \multicolumn{4}{|c|}{ schnelle Strömung } \\
\hline & \multicolumn{2}{|c|}{ Durchfluss $\left[\frac{\mathrm{ml}}{\mathrm{min}}\right]$} & \multicolumn{2}{|c|}{ Abweichung [\%] } & \multicolumn{2}{|c|}{ Durchfluss $\left[\frac{m l}{\min }\right]$} & \multicolumn{2}{|c|}{ Abweichung [\%] } \\
\hline & Ethanol & Wasser & Ethanol & Wasser & Ethanol & Wasser & Ethanol & Wasser \\
\hline X1Z1 & 11,2 & 8,7 & 7,69 & 0,63 & 91,35 & 111,84 & $-7,35$ & 3,35 \\
\hline $\mathrm{X} 1 \mathrm{Z} 2$ & 10,3 & 10,3 & $-0,80$ & 15,48 & 100,30 & 74,94 & 2,24 & $-44,24$ \\
\hline X1Z3 & 10,6 & 9,3 & 2,41 & 6,35 & 97,06 & 106,77 & $-1,02$ & $-1,24$ \\
\hline X1Z4 & 10,2 & 9,0 & $-1,94$ & 2,97 & 100,47 & 123,15 & 2,40 & 12,23 \\
\hline $\mathrm{X} 1 \mathrm{Z} 5$ & 10,6 & 9,8 & 2,04 & 11,61 & 96,62 & 107,50 & $-1,49$ & $-0,55$ \\
\hline $\mathrm{X} 1 \mathrm{Z6}$ & 9,9 & 9,1 & $-4,35$ & 4,84 & 97,06 & 111,54 & $-1,02$ & 3,09 \\
\hline $\mathrm{X} 1 \mathrm{Z7}$ & 10,5 & 10,5 & 1,52 & 17,42 & 82,35 & 109,69 & $-19,07$ & 1,46 \\
\hline $\mathrm{X} 1 \mathrm{Z} 8$ & 10,5 & 9,4 & 1,63 & 7,87 & 96,18 & 84,79 & $-1,95$ & $-27,49$ \\
\hline X1Z9 & 11,4 & 9,7 & 9,21 & 10,44 & 96,16 & 110,67 & $-1,97$ & 2,33 \\
\hline X2Z1 & 11,9 & 10,0 & 12,62 & 13,49 & 99,41 & 113,69 & 1,36 & 4,92 \\
\hline X2Z2 & 10,6 & 8,2 & 2,21 & $-6,39$ & 100,47 & 115,54 & 2,40 & 6,44 \\
\hline X2Z3 & 10,4 & 8,7 & 0,55 & 0,47 & 99,11 & 108,30 & 1,06 & 0,19 \\
\hline $\mathrm{X} 2 \mathrm{Z} 4$ & 10,8 & 8,1 & 4,20 & $-7,84$ & 93,66 & 112,39 & $-4,70$ & 3,82 \\
\hline X2Z5 & 9,5 & 8,0 & $-9,02$ & $-8,32$ & 92,81 & 118,18 & $-5,66$ & 8,53 \\
\hline X2Z6 & 10,6 & 8,8 & 2,39 & 1,47 & 92,29 & 112,05 & $-6,24$ & 3,53 \\
\hline $\mathrm{X} 2 \mathrm{Z7}$ & 10,0 & 7,7 & $-4,01$ & $-13,20$ & 96,90 & 112,78 & $-1,19$ & 4,16 \\
\hline $\mathrm{X} 2 \mathrm{Z} 8$ & 10,4 & 8,3 & 0,33 & $-4,92$ & 101,16 & 114,85 & 3,07 & 5,88 \\
\hline X2Z9 & 9,9 & 8,7 & $-4,82$ & 0,12 & 99,59 & 112,10 & 1,54 & 3,57 \\
\hline X3Z1 & 10,0 & 6,6 & $-3,17$ & $-31,21$ & 101,35 & 112,28 & 3,25 & 3,72 \\
\hline X3Z2 & 9,4 & 7,9 & $-9,90$ & $-10,11$ & 97,74 & 109,83 & $-0,32$ & 1,58 \\
\hline X3Z3 & 9,2 & 6,9 & $-12,23$ & $-25,48$ & 101,83 & 110,17 & 3,71 & 1,89 \\
\hline X3Z4 & 8,3 & 7,7 & $-24,63$ & $-13,59$ & 98,18 & 106,99 & 0,12 & $-1,03$ \\
\hline X3Z5 & 8,9 & 8,9 & $-16,58$ & 2,24 & 99,81 & 113,81 & 1,76 & 5,02 \\
\hline X3Z6 & 9,1 & 8,3 & $-13,28$ & $-4,19$ & 99,60 & 110,17 & 1,55 & 1,88 \\
\hline X3Z7 & 9,1 & 8,1 & $-13,68$ & $-7,24$ & 99,76 & 111,57 & 1,71 & 3,12 \\
\hline X3Z8 & 10,6 & 8,3 & 2,62 & $-5,27$ & 100,29 & 103,41 & 2,23 & $-4,53$ \\
\hline X3Z9 & 11,1 & 9,2 & 6,62 & 5,07 & 100,15 & 110,83 & 2,09 & 2,47 \\
\hline X4Z1 & 9,9 & 7,0 & $-4,22$ & $-24,12$ & 101,98 & 105,83 & 3,84 & $-2,14$ \\
\hline $\mathrm{X} 4 \mathrm{Z} 2$ & 10,7 & 8,2 & 3,15 & $-6,12$ & 99,62 & 101,15 & 1,57 & $-6,87$ \\
\hline X4Z3 & 10,5 & 8,6 & 1,40 & $-1,27$ & 100,13 & 106,09 & 2,07 & $-1,89$ \\
\hline $\mathrm{X} 4 \mathrm{Z} 4$ & 11,2 & 9,6 & 7,31 & 9,56 & 99,96 & 105,24 & 1,90 & $-2,72$ \\
\hline X4Z5 & 11,0 & 9,1 & 5,37 & 4,12 & 100,13 & 104,38 & 2,07 & $-3,55$ \\
\hline X4Z6 & 11,2 & 9,6 & 7,85 & 9,56 & 93,83 & 103,70 & $-4,51$ & $-4,23$ \\
\hline X4Z7 & 9,9 & 8,7 & $-4,18$ & $-0,12$ & 100,80 & 104,65 & 2,72 & $-3,29$ \\
\hline X4Z8 & 11,8 & 8,9 & 12,22 & 2,42 & 101,64 & 105,16 & 3,53 & $-2,79$ \\
\hline X4Z9 & 11,5 & 9,0 & 10,12 & 3,27 & 100,30 & 105,41 & 2,23 & $-2,55$ \\
\hline Mittel & 10,4 & 8,7 & - & - & 98,1 & 108,1 & - & - \\
\hline
\end{tabular}

Tabelle 5.1.: Gemessener Durchfluss bei langsamer und schneller Strömung 
Zeit zwischen diesen Messungen besonders lang ist. Die eigentlich konstant zu haltenden Durchflusswerte weichen um bis zu 30 \% von den Mittelwerten ab. Der Fehler ist aber vermutlich zum größten Teil bei der Durchflussmessung selbst zu suchen. Der Höhenunterschied der Flüsigkeitspegel betrug nur rund $10 \mathrm{~mm}$ und die Ablesefehler liegen bei bis zu $1 \mathrm{~mm}$. Damit ergibt sich allein aus dieser Fehlerquelle ein Fehler von bis zu $10 \%$. Weiterhin wurde vorausgesetzt, dass die Innenmaße der Voratsbehälter auf ganzer Höhe konstant bleiben. Der Hersteller gibt für die Innendurchmesser zwar Werte und grobe Fehlerschätzung an, garantiert diese Werte jedoch nicht. Eine Kontrolle der Werte ist durch die sehr engen Zugänge der Reservoirs unmöglich. Abweichungen sind also wiederum nicht auszuschließen. Der dritte wichtige Fehlerfaktor ist der Öffnungs- und Absperrvorgang. Die Absperrventile müssen gleichzeitig betätigt werden und möglichst schnell geöffnet und geschlossen werden. Tatsächlich brauchen beide Prozesse Zeit und werden niemals vollständig synchron durchgeführt.

\subsubsection{Dreidimensionale Konzentrationsverteilung im Multilaminationsmischer}

Die Konzentrationsverteilung von Ethanol bei der Mischung mit Wasser wurde bei zwei unterschiedlichen Strömungsbedingungen gemessen. Die Mischstrecke des Multilaminationsmischers wurde dabei wie beschrieben abgerastert. An jeder der 36 Messpositionen wurden 100 Intensitätsverteilungen des Ramanstreulichts von Ethanol aufgenommen. Durch diese Datenmenge ist es möglich, Mittelwerte und Standardabweichungen der Ethanolverteilung zu berechnen. Die Mittelwertbilder geben einen Überblick über den Strömungsverlauf. Bei stationären Strömungsbereichen bilden sich die Strukturen im Mittelwertbild deutlich aus, da sie sich während des Vorgangs nicht, bzw. kaum ändern. Die Betrachtung der Standardabweichung unterstützt diese Aussagen und kann zusätzliche Informationen über instationäre Bereiche in der Mischstrecke geben, die allein durch die Analyse der Mittelwerte nicht zu erkennen wären. Die Reynoldszahlen liegen bei der langsamen Strömung zwischen $\mathrm{Re}=100$ und $\mathrm{Re}=200$ und bei der schnellen zwischen $R e=1.000$ und $R e=2.000$. Bei der Berechnung der Reynoldszahlen ergeben sich die genannten Bereiche, da die eingestellten Strömungsgeschwindigkeiten nicht konstant sind und sich die Viskositäten der beiden Flüssigkeiten unterscheiden. Die Reynoldszahlen wurden mit einem hydraulischen Längenmaß berechnet, das der Breite der Auslasskanäle $(1 \mathrm{~mm})$ entspricht, da diese die Strömung zu Beginn der Messstrecke dominieren. Da die typischen Strömungsgeschwindigkeiten im weiteren Verlauf der Mischstrecke deutlich sinken, liegen die berechneten Reynoldszahlen eher hoch. Das bedeutet, dass auch im Fall der schnelleren Strömung keine Turbulenz auftreten dürfte, da der klassische Grenzbereich bei etwa $R e=2300$ nicht erreicht wird. Weitere Informationen erhält man aus den Einzelbildern, die aufgrund der sehr kurzen Belichtungszeiten von ca. 6 ns (Laserpulsdauer) sehr präzise Momentaufnahmen der Ethanolverteilung enthalten. Wie hoch die Qualität des Mischvorgangs ist, lässt sich letztendlich nur anhand der Einzelbilder erkennen, da Mittelwerte auch dann einen perfekten Wert ergeben können, wenn die Fluide noch stark getrennt vorliegen. 


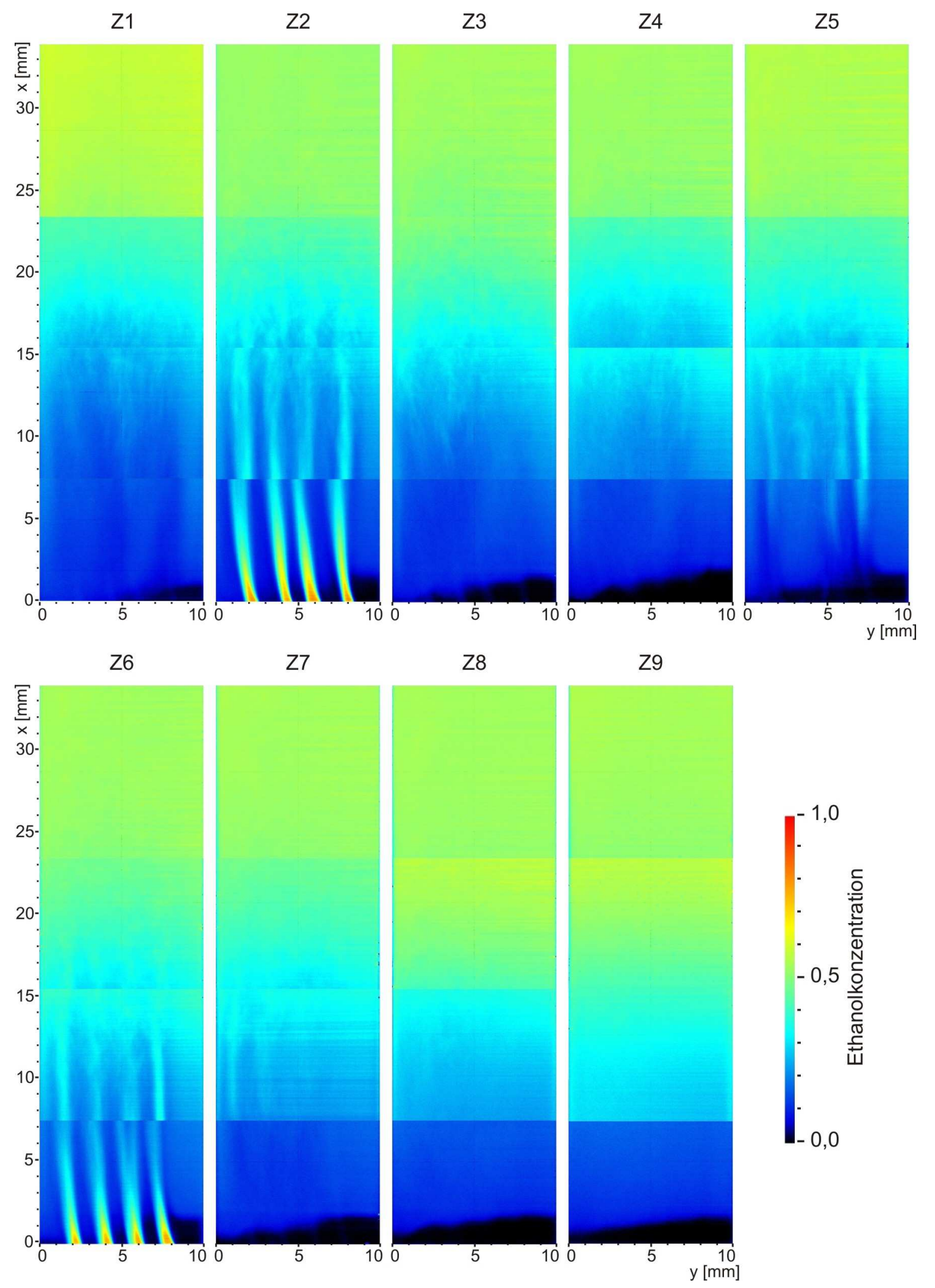

Abb. 5.19.: Montage der Mittelwertbilder an den Positionen X1-X4 nach Vermessung einer langsamen Strömungsgeschwindigkeit des Multilaminationsmischers 
Z1

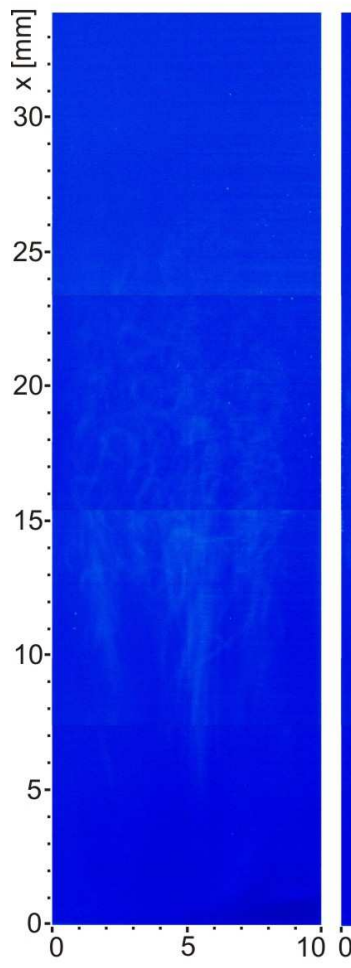

Z6

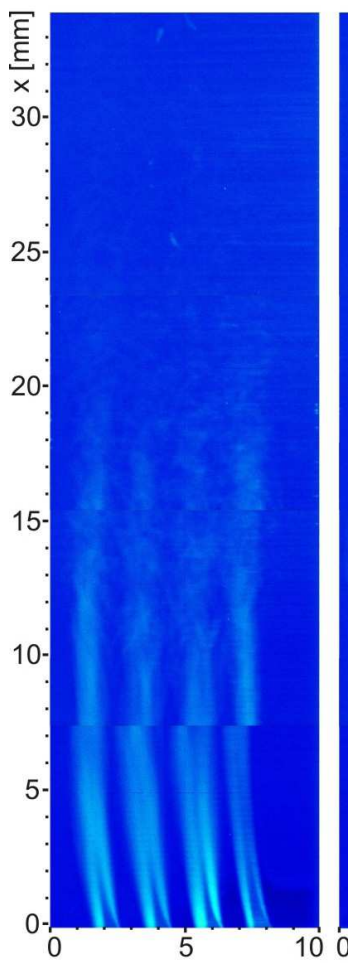

Z2

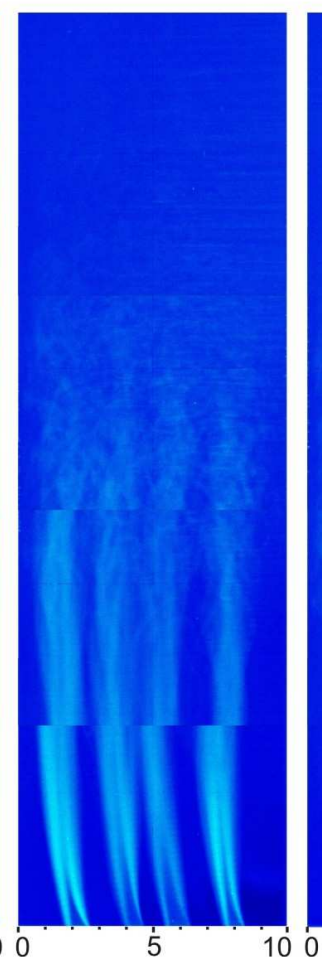

$Z 7$

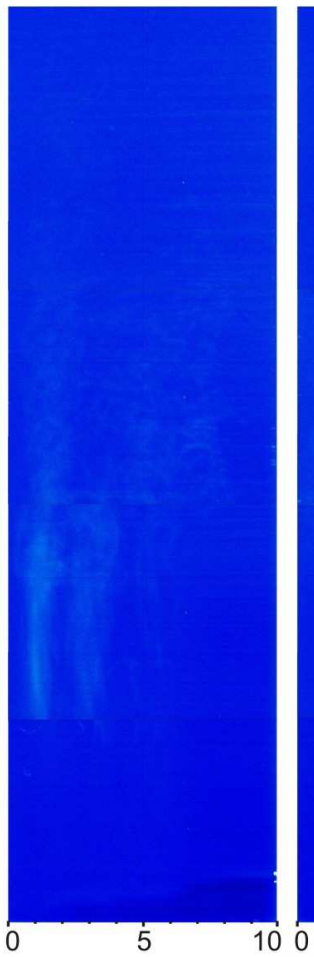

Z3

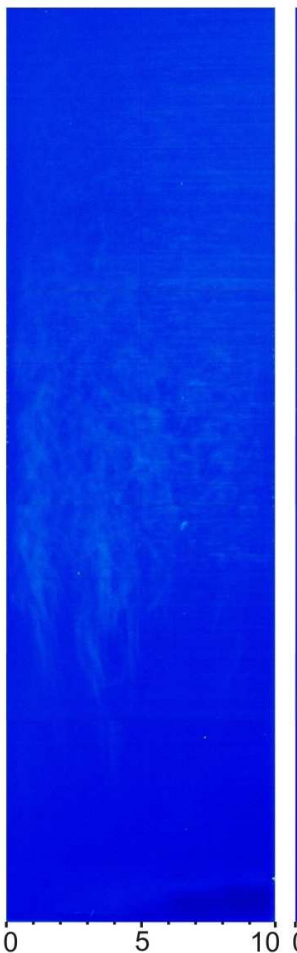

Z8

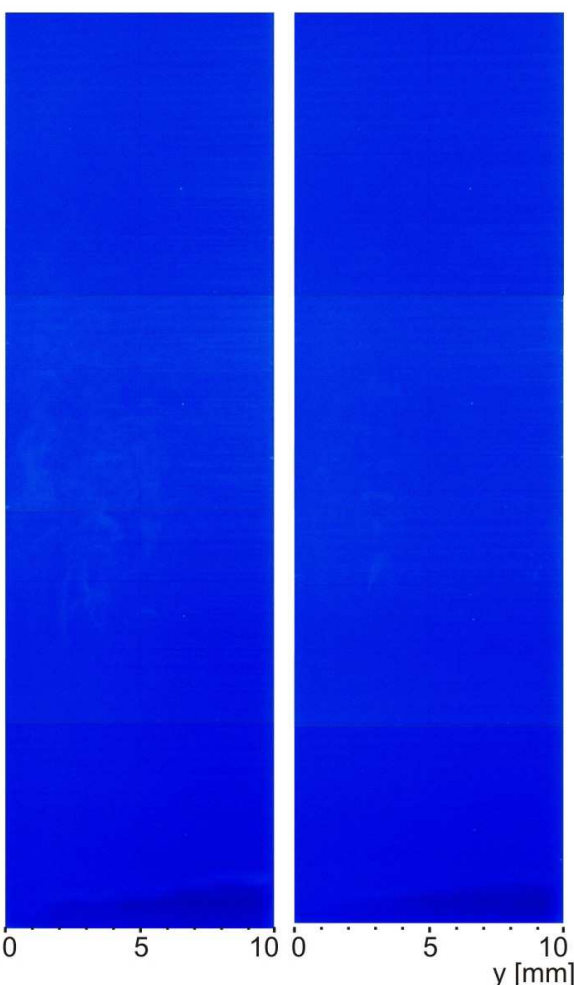

Z5

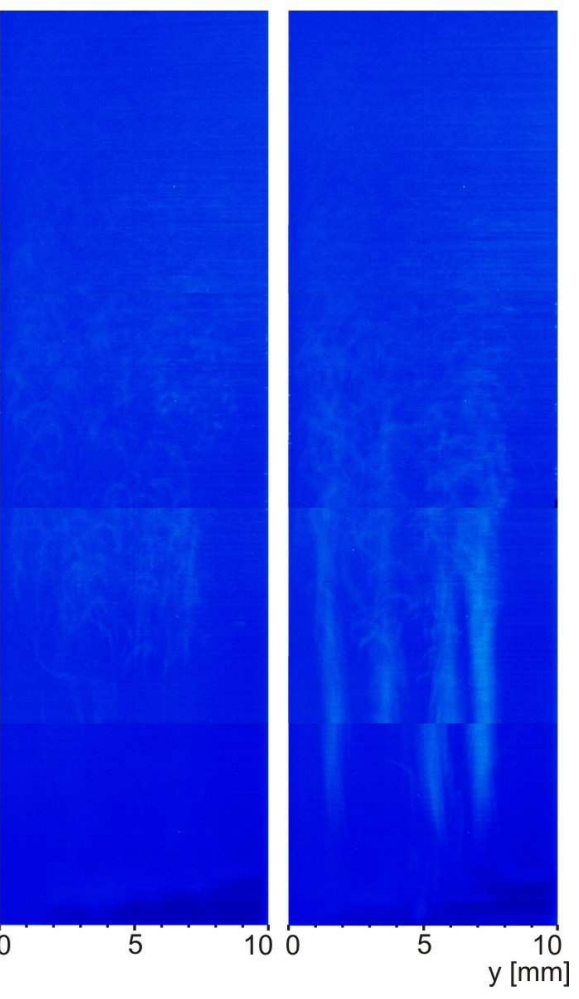

Z9

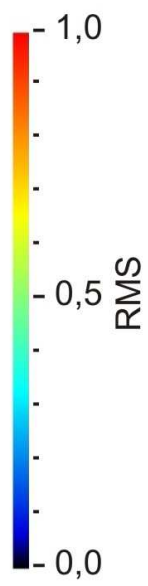

Abb. 5.20.: Montage der Bilder der Standardabweichungen an den Positionen X1-X4 nach Vermessung einer langsamen Strömungsgeschwindigkeit des Multilaminationsmischers 


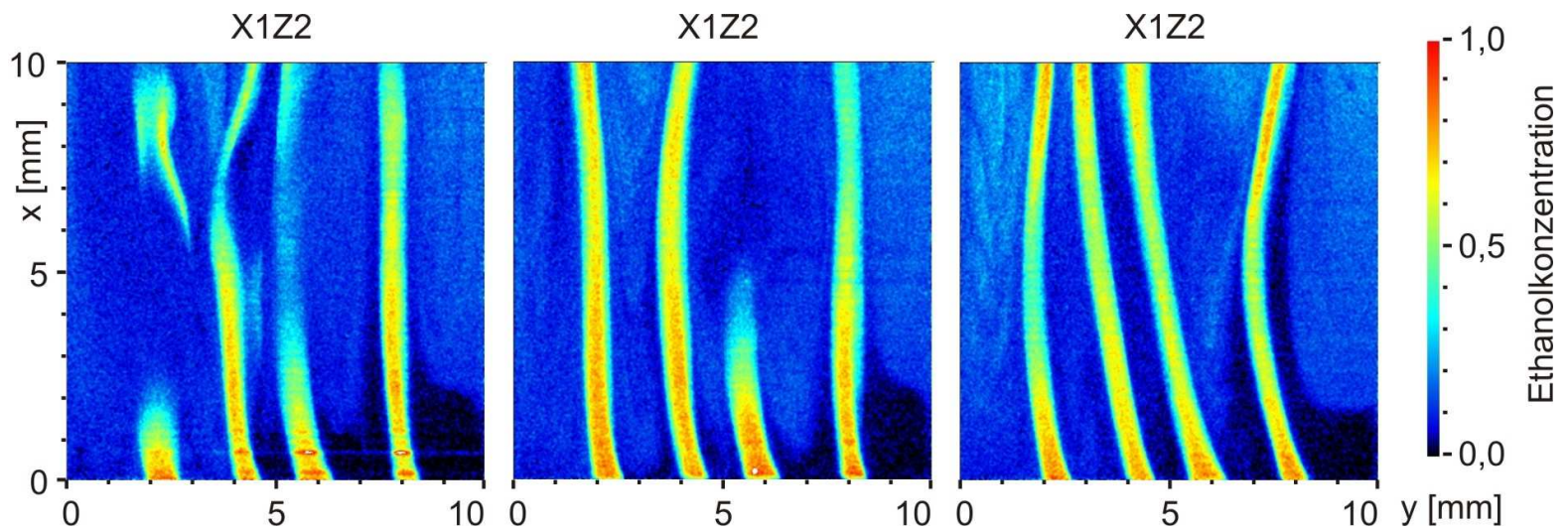

Abb. 5.21.: Momentaufnahmen der Ethanolverteilung am unteren Ende der Mischstrecke des Mischers bei der langsamen Strömung. Die Ethanolfäden schwanken nach Austritt aus den Zuläufen stark hin und her.

Die Abbildungen 5.19 und 5.23 zeigen Montagen der Mittelwertbilder der Ethanolverteilung in allen vermessenen Positionen. Die Standardabweichungen sind in den Abbildungen 5.20 und 5.24 auf die gleiche Weise dargestellt. Die vier Aufnahmen in x-Richtung wurden in den Abbildungen aneinander gereiht, um ein durchgängiges Bild zu erzeugen. Jede Position in z-Richtung ist in einer einzelnen Spalte dargestellt. Die Zuleitungskanäle der beiden Flüssigkeiten sind wie in Kapitel 2.2 beschrieben um $45^{\circ}$ gegen die Austrittsfläche geneigt. Bei allen im Folgenden gezeigten Aufnahmen waren die Ethanolauslässe nach links gerichtet und die Wasserauslässe nach rechts. Zunächst soll nun detaillierter auf die Beobachtungen der langsamen Strömung eingegangen werden.

Im Fall der langsamen Strömung zeigen die Mittelwertbilder (Abbildung 5.19) insgesamt eine kontinuierliche Zunahme der Ethanolkonzentration von unten nach oben. Im unteren Bereich liegt die Konzentration nahe Null, ganz oben liegt sie bei etwas über $50 \%$. Das Ethanol fließt zunächst unter einem Winkel aus den Kanälen und bewegt sich fadenförmig durch das umgebende Fluid nach oben. Dies ist in den Ebenen Z2 und Z6, in denen die Auslässe des Ethanol liegen, gut zu erkennen (Lage der Positionen in Abbildung 5.18). Die Ethanolfäden schwanken stark, dies ist vor allem in den Einzelbildern in Abbildung $5.21 \mathrm{zu}$ erkennen, die die Ethanolverteilung zu verschiedenen Zeitpunkten an derselben Stelle (X1Z2) zeigen. Trotzdem sind die Fäden auch in den Mittelwertbildern (Abbildung 5.19) deutlich zu erkennen, ihr Schwerpunkt verläuft also dauerhaft in einem schmalen Streifen. Im untersten Bereich, nah an den Auslässen, liegt die Standardabweichung innerhalb der Fäden auf niedrigem Niveau, während die Außenbereiche sehr hohe Werte zeigen. Dies entspricht genau dem Ergebnis, das bei einem starken örtlichen Schwanken der Fäden zu erwarten ist. Das Wasser tritt in den Ebenen Z4 und Z8 aus und zeigt dabei ein deutlich anderes Verhalten als das Ethanol. Es scheint nicht nach oben zu fließen, sondern sammelt sich zunächst in einer Schicht am unteren Rand der Mischstrecke (schwarzer Bereich bis etwa $\mathrm{x}=2 \mathrm{~mm}$ ). Diese Schicht wird nach rechts 


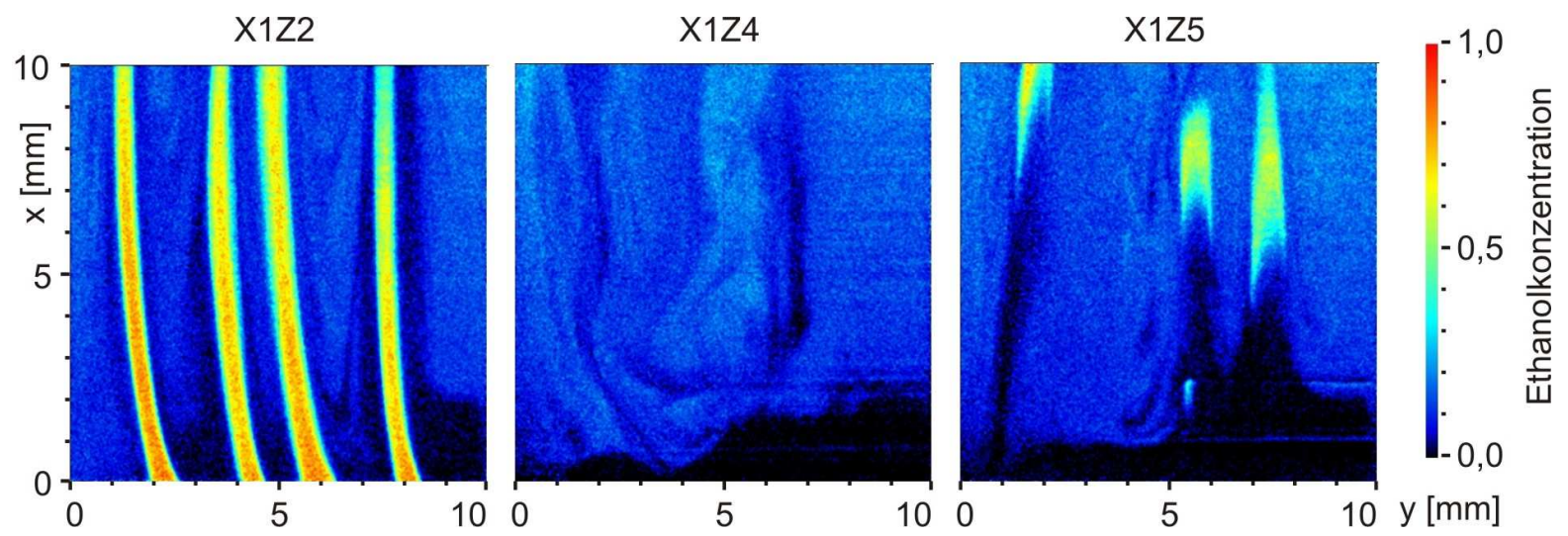

Abb. 5.22.: Momentaufnahmen der Ethanolverteilung am unteren Ende der Mischstrecke des Mischers bei der langsamen Strömung. Die Strömung des Wassers nach oben geschieht vor allem durch in der Nähe aufsteigendes Ethanol.

dicker, was aller Wahrscheinlichkeit nach an dem nach rechts gerichteten Fluidauslass liegt. Die Wasserschicht erstreckt sich über alle Ebenen, ihr Umfang nimmt von den vorderen Ebenen Z1 bis zur hintersten Ebene Z9 zu. Diese Asymmetrie resultiert aus der Tatsache, dass die Ethanol- und Wasserauslässe nicht symmetrisch angeordnet sind. Ethanol tritt eher im vorderen Bereich aus (Z2 und Z6), während das Wasser im hinteren Bereich in die Mischkammer fließt (Z4 und Z8). Das Wasser steigt nicht in geordneten Fäden nach oben. Einzelne Bereiche nahezu reinen Wassers, wie die Momentaufnahme in Abbildung 5.22 an Position X1Z4 zeigt, steigen vermutlich nur deshalb nach oben, da in einer angrenzenden Schicht ebenfalls Ethanol aufsteigt, das in dieser Schicht nicht erfasst wurde. Weitere Momentaufnahmen in den Positionen X1Z2 und X1Z5 lassen erkennen, dass Wasser aus dem am Boden befindlichen Bereich durch das aufsteigende Ethanol mitgerissen wird. Das Einzelbild in Position X1Z5 zeigt, dass die Ethanolfäden sich sogar in die benachbarten Ebenen hinein bewegen. Im Nachlauf des Ethanols fließt, wie in Ebene Z2, Wasser nach oben.

Das unterschiedliche Verhalten der beiden Flüssigkeiten ist auf ihre spezifischen Dichten zurückzuführen. Diese liegt bei Wasser unter Normalbedingungen bei $1 \mathrm{~g} / \mathrm{cm}^{3}$ und beträgt beim Ethanol 0,79 g/ $\mathrm{cm}^{3}$. Der Dichteunterschied von $21 \%$ bestimmt im Experiment das Verhalten der beiden Flüssigkeitsströmungen. Während Ethanol sehr schnell aufsteigt, sammelt sich das Wasser aufgrund seiner höheren Dichte am Grund. Der leicht höhere Durchfluss des Ethanols (siehe Tabelle 5.1) verschärft diese Situation, dürfte aber insgesamt eine untergeordnete Rolle spielen.

Die Gemischbildung bei schnellerer Strömung unterscheidet sich von der bei der langsameren Strömung deutlich. Die Strömungsgeschwindigkeiten liegen ca. zehn mal höher als zuvor. Dadurch schießen beide Flüssigkeiten geradezu aus den Zulaufkanälen. Abbildung 5.23 zeigt diese Gegebenheit in den Messebenen oberhalb der Auslässe, also Z2 und Z6 für Ethanol, bzw. Z4 und Z8 für Wasser, sehr deutlich. Beide Flüssigkeiten treten 


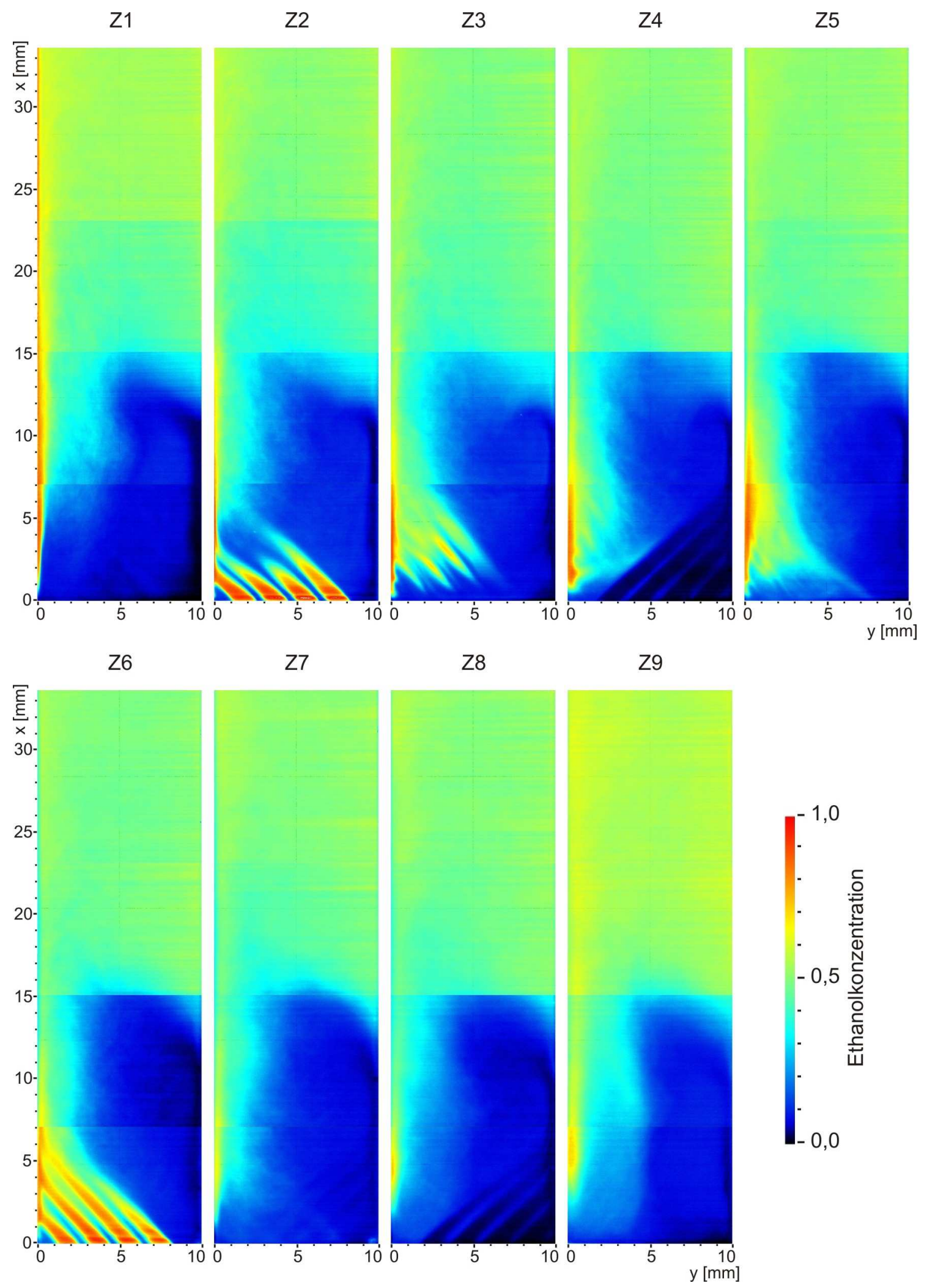

Abb. 5.23.: Montage der Mittelwertbilder an den Positionen X1-X4 nach Vermessung einer schnellen Strömungsgeschwindigkeit des Multilaminationsmischers 

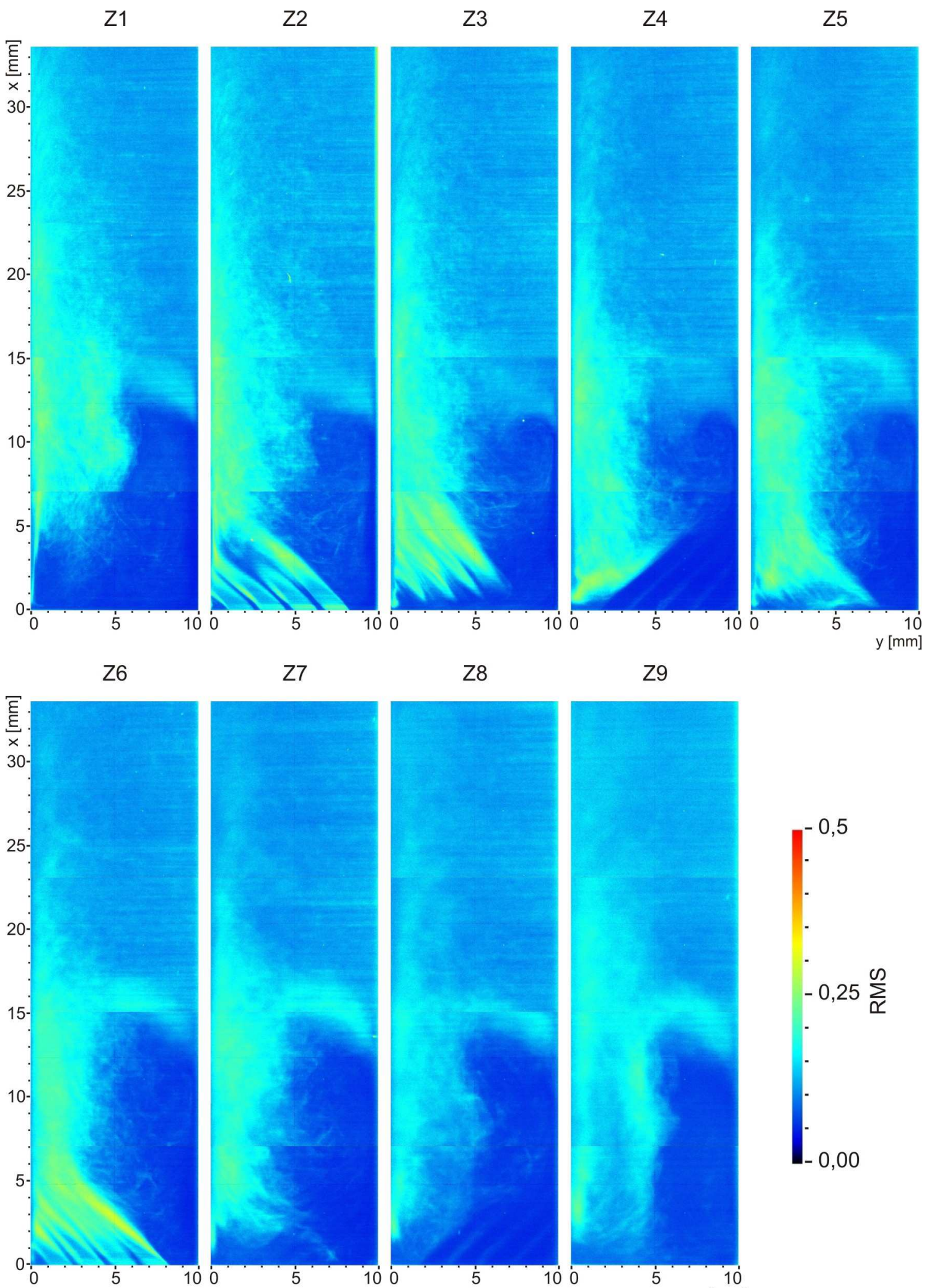

$\mathrm{Z7}$

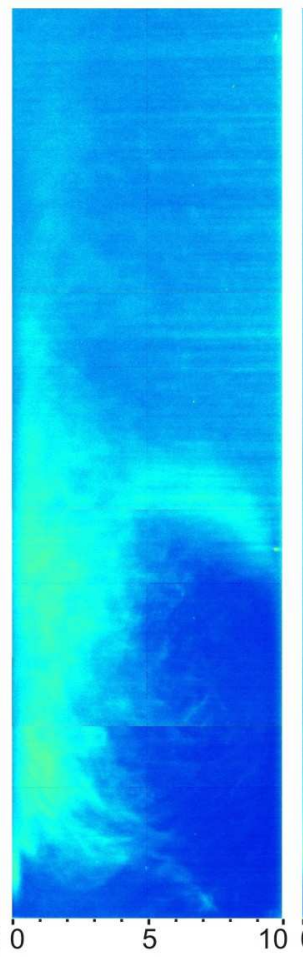

Z8

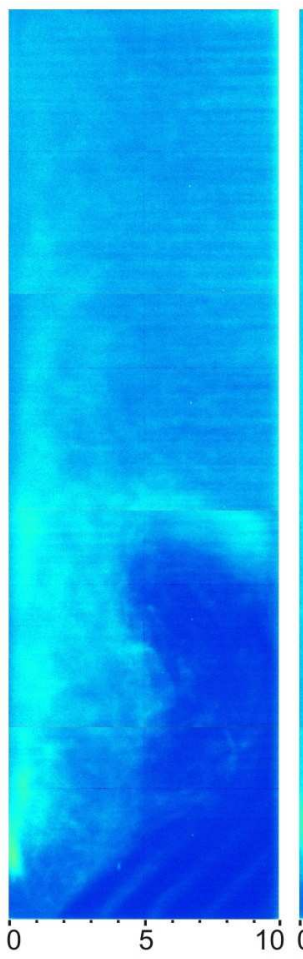

Z9

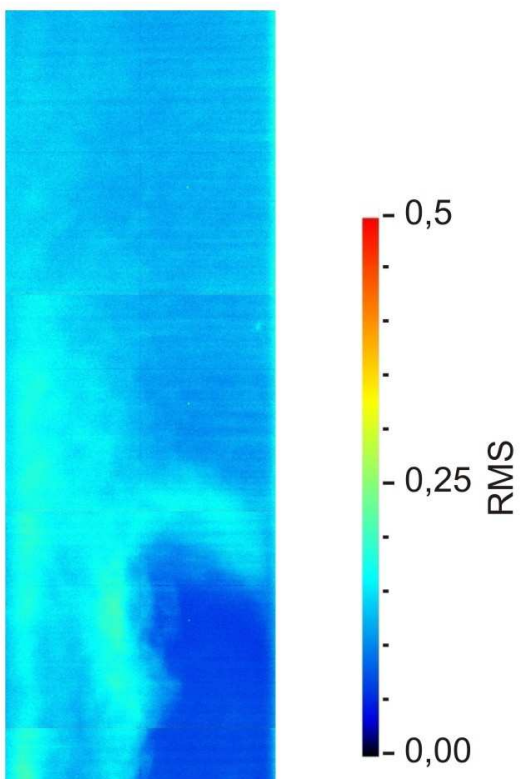

Abb. 5.24.: Montage der Bilder der Standardabweichungen an den Positionen X1-X4 nach Vermessung einer schnellen Strömungsgeschwindigkeit des Multilaminationsmischers 


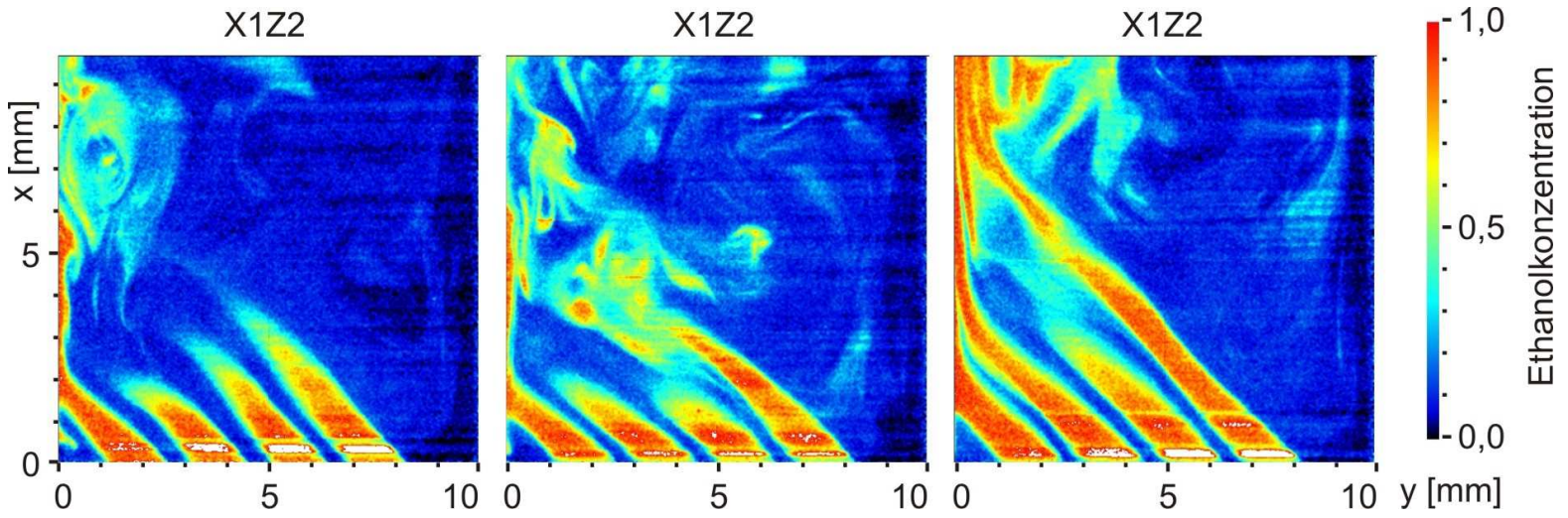

Abb. 5.25.: Momentaufnahmen der Ethanolverteilung am unteren Ende der Mischstrecke des Mischers bei der schnellen Strömung. Die Ethanolfäden schwanken nach Austritt aus den Zuläufen stark hin und her und scheinen schnell zu zerfallen.

unter einem Winkel von $45^{\circ}$ zur Mischkammerachse aus, der von den Zuläufen vorgegeben wird. Es existieren durchgehende Ethanol- bzw. Wasserfäden bis an die Wände der Mischkammer. An den Wänden fließen die Flüssigkeiten auseinander, so dass in allen Ebenen der Mittelwertbilder eine dünne Schicht der reinen Flüssigkeiten deutlich zu erkennen ist. An der linken Wand liegt die Ethanolkonzentration jeweils nahe eins und an der rechten Wand nahe Null. Die Bilder der Standardabweichung in Abbildung 5.24 zeigen für die Ethanolfäden, wie bei der langsamen Strömung, im Inneren einen niedrigen Wert und an den Rändern einen deutlich höheren. Der Verlauf der Fäden ändert sich stark, wie auch aus Abbildung 5.25 ersichtlich ist. Die Abbildung zeigt drei verschiedene Momentaufnahmen an derselben Position X1Z2. Im Gegensatz zur langsamen Strömung bilden sich im Mittelwertbild (Abbildung 5.23) bei schneller Strömung deutlich erkennbare Wasserfäden als dunkle Bereiche in der Ethanolkonzentrationsverteilung aus. Die drei Momentaufnahmen in Abbildung 5.26 zeigen genauere Details der Ethanolverteilung. Es tritt in Ebene Z2 aus, strömt dann jedoch weiter in Richtung Mitte der Mischstrecke. Dies ist in Ebene Z3 zu erkennen, da die Ethanolfäden diese Ebene durchqueren. Die Strömungssituation ist insgesamt stark instationär und es sind in den Einzelbildern bereits direkt hinter dem Eintritt in die Mischkammer viele einzelne Bereiche erkennbar, die sich von den Fäden lösen.

Global unterscheidet sich der Konzentrationsverlauf ebenfalls deutlich von der langsameren Strömung. Von unten nach oben nimmt die Konzentration des Ethanols nicht kontinuierlich zu, sondern es existieren zwei deutlich trennbare Bereiche, deren Grenze in allen Ebenen Z1 bis Z9 bei etwa $\mathrm{x}=15 \mathrm{~mm}$ liegt. Diese Grenze ist in den Mittelwertaufnahmen in Abbildung 5.23 deutlich zu erkennen. Unterhalb der Grenze sind Ethanol und Wasser noch deutlich getrennt, während darüber beide Flüssigkeiten bei einer Ethanolkonzentration von $50 \%$ im zeitlichen Mittel gleich verteilt sind. Im unteren Bereich liegt die Konzentration auf der linken Seite über $50 \%$, ist also vorwiegend von Ethanol besetzt und die Konzentration auf der rechten Seite liegt unter $50 \%$, dort findet man 


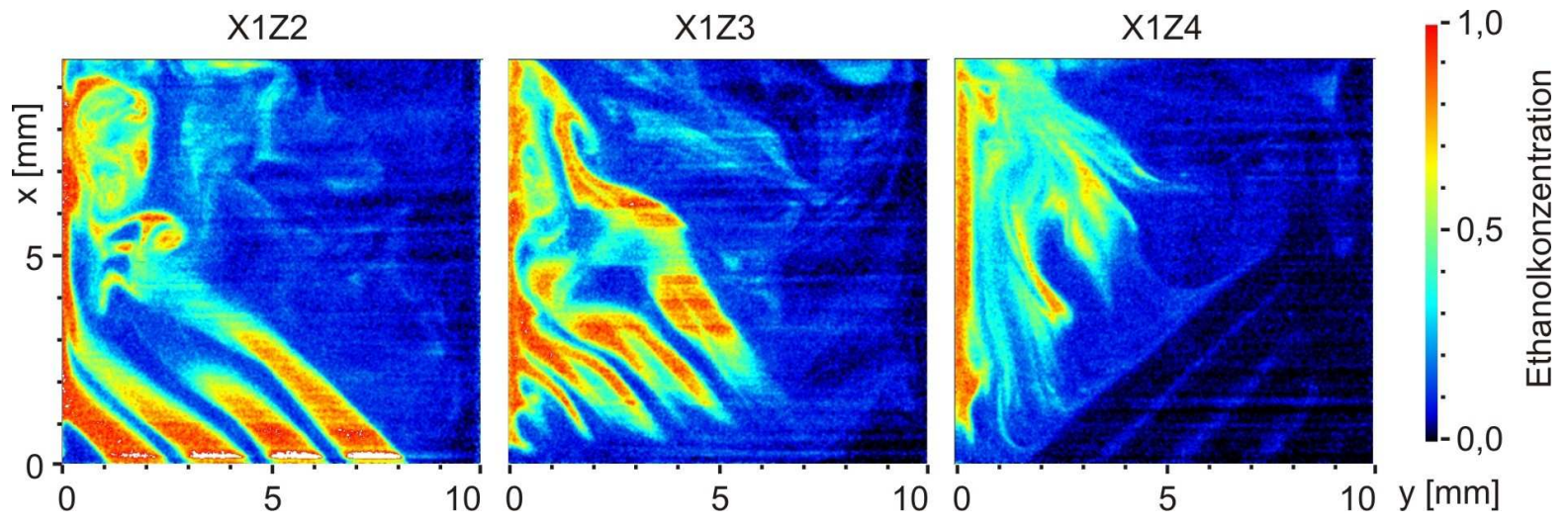

Abb. 5.26.: Momentaufnahmen der Ethanolverteilung am unteren Ende der Mischstrecke des Mischers bei der schnellen Strömung. Der tendenzielle Verlauf der Ethanolfäden durch mehrere Schichten des Mischers ist gut zu erkennen.

also vorwiegend Wasser. Dabei liegt der Übergang von vorwiegend Ethanol zu vorwiegend Wasser so, dass das Wasser einen erheblich größeren Bereich einnimmt, der je nach betrachteter Ebene bis $\mathrm{y}=4 \mathrm{~mm}$ oder sogar $\mathrm{y}=3 \mathrm{~mm}$ reicht, also etwa zwei Drittel der Fläche einnimmt. Das Ethanol fließt aufgrund seiner geringeren Dichte deutlich schneller nach oben. Um diese Vermutung zu bestätigen, wären gleichzeitige Geschwindigkeitsmessungen notwendig, die derzeit nicht vorliegen. Aus den Bildern der Standardabweichung in Abbildung 5.24 lässt sich folgern, dass die Vermischung der beiden Flüssigkeiten, trotz der im Mittel bestehenden Gleichverteilung am Ende der Mischstrecke, nicht besonders gut gelungen ist. Die Werte der Standardabweichung sind im oberen Bereich vor allem an der linken Wand noch immer hoch. Dies bedeutet stark schwankende Konzentrationswerte und damit eine schlechte Vermischung. Einzelbilder an verschiedenen Positionen im oberen Bereich der Mischstrecke bestätigen diese Aussagen deutlich. Abbildung 5.27 zeigt auch in der höchsten Messposition noch immer deutliche Bereiche der nahezu ungemischten Flüssigkeiten. Die Umgebung dieser kaum gemischten Bereiche ist dagegen sehr gut vermischt. Interessant ist, dass sich die meisten Ethanol-Bereiche zwar an der linken Wand nach oben bewegen, Wasser in höherer Konzentration aber nicht an der rechten Wand vorzufinden ist. Auch hier scheint letztendlich der Auftrieb des Ethanols für die Gestaltung der Strömung eine entscheidende Rolle zu spielen. Das Wasser wird im oberen Bereich wiederum vom Ethanol mitgerissen.

Besonders interessant ist der Übergang bei $\mathrm{x}=15 \mathrm{~mm}$ vom schlecht gemischten Bereich hinter den Auslässen zum Bereich gleichmäßiger mittlerer Konzentrationsverteilung oberhalb. Die hohen Werte der Standardabweichung in Abbildung 5.24 zeigen, dass die Konzentrationsschwankungen am Übergang besonders stark sind. Dieser Bereich ist deutlicher in den Momentaufnahmen in Abbildung $5.28 \mathrm{zu}$ erkennen. Am unteren Rand der Aufnahmen befinden sich immer wieder Regionen mit nahezu reinem Wasser, die in einen Bereich relativ gut gemischter Flüssigkeit hineinragen. Das obere Ende des Wasserbereichs ändert sehr stark seine Größe, Form und Position. Diese lokal schwankenden 


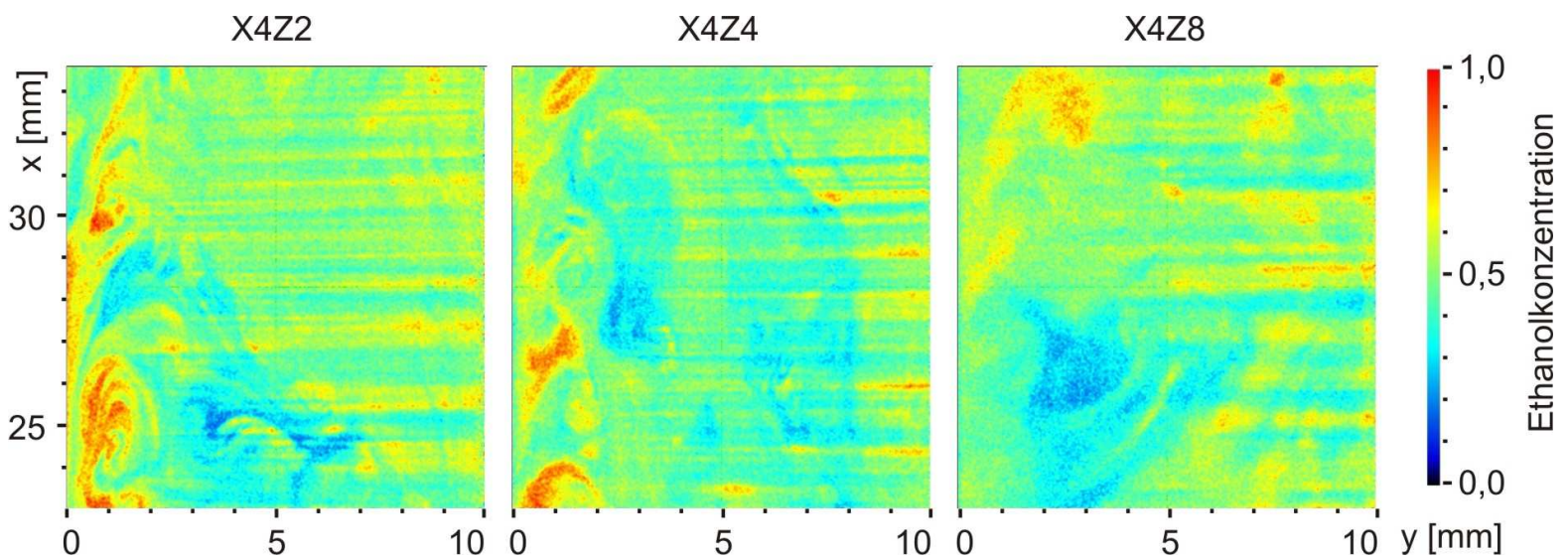

Abb. 5.27.: Momentaufnahmen der Ethanolverteilung am oberen Ende der Mischstrecke des Mischers bei der schnellen Strömung. Ungemischte Bereiche nahezu reinen Ethanols und Wassers sind deutlich zu erkennen.

großen Konzentrationsgradienten sorgen für den hohen Wert der Standardabweichung an dieser Position. An dieser Grenze werden vor allem auf der linken Seite immer wieder Bereiche nahezu reinen Wassers aus der unteren Region gerissen und nach oben getrieben. Die Situation lässt starke Scherkräfte zwischen schnell auftreibendem Ethanol links und dem deutlich dichteren Wasser rechts vermuten. Eine genauerere Untersuchung der Gemischbildungsdynamik wäre durch ergänzende Geschwindigkeitsmessungen möglich.

Vor allem bei der Begutachtung der Momentaufnahmen in Abbildung 5.27 und 5.28 fallen deutlich horizontale helle und dunkle Streifen auf. Außerdem ist in Abbildung 5.27 eine erhebliche Verschlechterung der Bildschärfe zu erkennen. In der vordersten Schicht Z2 sind noch alle Details sehr scharf umrissen, in Schicht Z8 dagegen sind die Grenzen der Bereiche wesentlich verwaschener. Beide Phänomene lassen sich durch unterschiedliche Brechungsindizes erklären. An den Grenzen der Bereiche liegen starke Brechungsindexgradienten vor, die das Laserlicht fokussieren. Zudem ist durch den Blick durch das nicht vollständig gemischte Medium keine scharfe Abbildung möglich. Vor dem Experiment ist eine sehr gründliche Brechungsindexanpassung der beiden Flüssigkeiten, wie in Kapitel 5.7.1 beschrieben, durchgeführt worden. Die Beobachtungen zeigen, dass Brechungsindexunterschiede trotz Anpassung immer noch auftreten. Es ist aber auch deutlich erkennbar, dass die Unterschiede sich auf dem Weg nach oben mehr und mehr auswirken. Eine mögliche Erklärung dafür ist das vermehrte Auftreten von kleinen Bereichen nahezu reiner Stoffe, das auch bei nur sehr geringen, nach der Brechungsindexanpassung noch verbleibenden Brechungsindexunterschieden zu einer Fokussierung der Laserstrahlung führen kann. Gegen diese These spricht jedoch, dass die Einzelbilder bereits ganz unten (Abbildung 5.26) starke Konzentrationsgradienten zeigen, die mit entsprechend hohen Brechungsindexgradienten einhergehen müssten. Da in der unteren Region ein Bereich einer Flüssigkeit für gewöhnlich von der anderen Flüssigkeit in nahezu reiner Form umgeben ist müssten die Gradienten besonders groß sein. Die Fokussierungen bzw. 

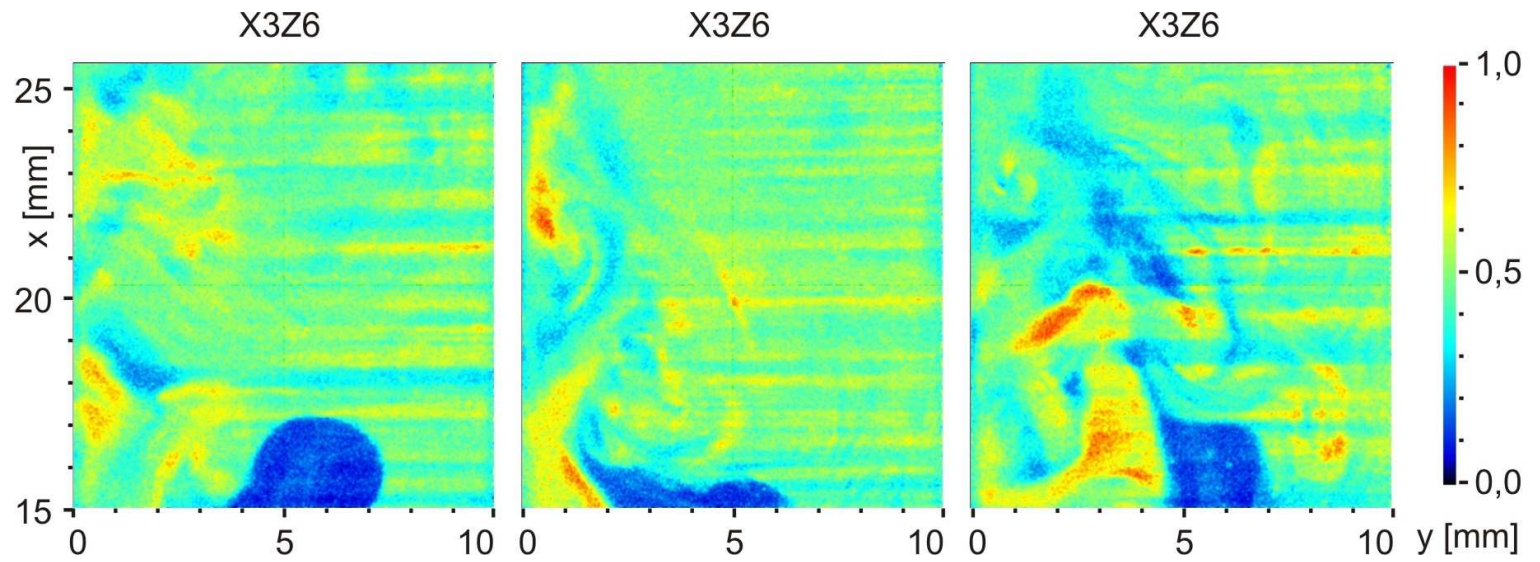

Abb. 5.28.: Momentaufnahmen der Ethanolverteilung oberhalb $\mathrm{x}=15 \mathrm{~mm}$ bei der schnellen Strömung. Am unteren Bildrand liegt der Übergang von einem Bereich nahezu reinen Wassers zu gut gemischtem Fluid.

die Unschärfe sollte also nach oben, entgegen der These, eher abnehmen oder zumindest gleich bleiben. Die Überlegung kann damit als wiederlegt gelten. Eine weitere mögliche Erklärung ist, dass die Brechungsindexunterschiede erst im Verlauf der Gemischbildung und damit vermehrt im oberen Teil der Mischkammer entstehen. Durch die Vermischung von Ethanol und Wasser wird ein energetisch günstigerer Zustand erreicht und damit Wärme freigesetzt. Dies geschieht verstärkt in Regionen starker Vermischung und damit an den Rändern der Bereiche nahezu reiner Flüssigkeiten. Bereits geringe Temperaturunterschiede verändern den Brechungsindex des Mediums. Durch das vermehrte Auftreten von kleinen Bereichen der Reinstoffe im oberen Teil der Mischkammer werden dort auch vermehrt Brechungsindexunterschiede durch die vom Mischprozess generierten Temperaturgradienten generiert. Aus diesen Überlegungen kann man folgern, dass sich Brechungsindexunterschiede nicht umgehen lassen, wenn sich die Temperatur bei der Mischung ändert. Dies ist bei den meisten Mischungen von Flüssigkeiten gegeben.

\subsection{Fazit}

Das Konzept der planaren Ramanstreuung zur quantitativen Aufzeichnung von Spezieskonzentrationen konnte erfolgreich in ein Messverfahren umgesetzt werden. Wichtige Schritte sind die optimale Ausnutzung der geringen Ramanintensitäten und die Auswahl eines geeigneten Ramanfilters. Die genaue Kenntnis der Ramanspektren aller verwendeten Flüssigkeiten ist für die Filterwahl unabdingbar. Ein Verfahren zur Berechnung von Volumenkonzentrationen aus den aufgenommenen Intensitätsverteilungen des Ramanstreulichts wurde entwickelt und validiert. Während der Entwicklung wurden verschiedene Fehlerquellen identifiziert und resultierende Fehler korrigiert. Vor allem Fehler, die auf zeitlich und örtlich schwankende Laserintensitäten zuückzuführen sind, konnten um- 
fassend ausgeglichen werden. Eine hohe quantitative Genauigkeit des Verfahrens konnte nachgewiesen werden. Bei der Überprüfung der Kalibration wurde ein Fehler von maximal $1 \%$ festgestellt. Die zweidimensionale Messung erreicht aufgrund der fehlenden räumlichen und zeitlichen Integration geringere Genauigkeitsbereiche, die abgeschätzten Fehler liegen insgesamt immer noch unter 4-5\%. Lediglich die Abweichungen, die durch die Brechungsindexunterschiede entstehen, können lokal zu bedeutend höheren Fehlern führen. Mit dem bestehenden experimentellen Aufbau und den zugehörigen Berechnungsverfahren wurden systematische Messungen in einem Mikromischer durchgeführt, mit denen detaillierte Überlegungen zu den zugrunde liegenden Gemischbildungsvorgängen angestellt werden konnten.

Die Mischung funktioniert bei den beiden untersuchten Durchflussraten relativ schlecht. Dies ist aber aufgrund des einfachen Aufbaus des hier verwendeten Mischerprototypen nicht verwunderlich. Dieser Mikromischer ist einfach konstruiert, um experimentelle Daten zu generieren, die eine Verifikation numerischer Simulationen ermöglichen, die wiederum nur für eine moderate Anzahl von Strömungsaustritten realisiert werden können. Aus dem Vergleich von Experiment und numerischer Simulation sollen Erkenntnisse gewonnen werden, die eine Optimierung der Mischergeometrie ermöglichen.

Durch die gleichzeitige Messung von Geschwindigkeitsfeldern wäre es möglich, umfangreiche weitere Schlüsse zu ziehen, und ein tiefgreifenderes Verständnis der Gemischbildungsvorgänge zu erlangen. Daher wäre zukünftig eine Kombination der beiden vorgestellten Messverfahren und eine dann mögliche simultane Messung von Konzentrationsverteilung und Geschwindigkeitsfeld ausgesprochen hilfreich. 


\section{Zusammenfassung und Ausblick}

Im Verlauf der Arbeiten wurden zwei neue Messverfahren für mikrofluidische Mischsysteme entwickelt und getestet. Die „Molecular Tagging Velocimetry“ konnte erfolgreich zu einem zweidimensional ortsaufösenden Geschwindigkeitsmessverfahren für die Mikrofluidik erweitert werden. Eine neue Markierungstechnik auf Basis der strukturierten Belichtung eines so genannten „caged dyes" ermöglichte es, Markierungen dauerhaft in ein Fluid zu schreiben. Durch Abbildung einer leicht modifizierbaren Maske ließ sich die Form der Markierung nahezu beliebig verändern und an die Geometrie des zu untersuchenden mikrofluidischen Mischsystems sowie die zu erwartenden Strömungsgeschwindigkeiten anpassen. Die Bewegung und Verformung der Markierung, die durch die Mikroströmung induziert wurde, konnte anschließend mit einer Kamera durch Anregung der Fluoreszenz des Farbstoffes beliebig oft ausgelesen werden. Die damit gewonnenen Bildserien wurden mit einer, an die Randbedingungen mikrofluidischer Strömungen angepassten, Variante der Methode des optischen Flusses ausgewertet und Geschwindigkeitsfelder der Strömung extrahiert. Dabei war es insbesondere notwendig, die Diffusion und Taylor-Dispersion, die durch die kleinen Kanaldimensionen hervorgerufenen wird, zu berücksichtigen, da diese physikalischen Vorgänge in Mikroströmungen dominieren. Damit konnten Fehler, die ohne eine derartige Anpassung der Algorithmen auftreten würden, korrigiert werden. Anhand von Kontrollmessungen in einem Modellkanal wurde gezeigt, dass die Messtechnik in der Lage ist, sehr genaue Messungen von Geschwindigkeitsfeldern mit einem mittleren Fehler von $4 \%$ durchzuführen. Ebenfalls durchgeführte Messungen des Geschwindigkeitsfeldes in der komplexeren Geometrie eines flächigen Mikromischers entsprachen auch qualitativ dem erwarteten Verlauf.

Durch die Entwicklungen konnten erstmalig quantitative zweidimensionale Geschwindigkeitsmessungen auf Grundlage der MTV im Bereich der Mikrofluidik durchgeführt werden. Insbesondere die neuartige Markierungstechnik, die keinen optischen Zugang von der Seite der Mikrokanäle erfordert, und die speziellen Anpassungen der Auswertealgorithmen unterscheiden sich deutlich von bisher etablierten Verfahren. In vielen Fällen wird der Einsatz der MTV als zweidimensionale Geschwindigkeitsmesstechnik in Mikrosystemen aufgrund dieser Neuerungen erst ermöglicht.

Auch die Bestimmung zweidimensionaler Konzentrationsverteilungen molekularer Spezies in Mikroströmungen konnte auf Grundlage der spontanen Ramanstreuung erfolgreich in ein Messverfahren umgesetzt werden. Bei Kenntnis der Ramanspektren aller am Gemischbildungsprozess beteiligten Flüssigkeiten ist es prinzipiell möglich, Transmissionsfilter auszuwählen, die nur das Ramanstreulicht einer einzelnen Spezies transmittieren. Durch Abbildung des transmittierten Lichts auf eine empfindliche Kamera kann so ein flächiges Abbild der quantitativen Verteilung der entsprechenden Spezies realisiert werden. In der Praxis ist man jedoch häufig mit der Situation konfrontiert, 
dass einerseits stark überlappende Spektren auftreten können, und anderseits die Signalintensitäten von spektral freien Ramanbanden der zu untersuchenden Spezies so gering sind, dass eine quantitative Detektion schwierig ist. Trotz dieser Herausforderungen konnte in dieser Arbeit der Mischprozess am Modellsystem Ethanol/Wasser quantitativ visualisert werden. Für derartige binäre Mischungen wurde eine Auswertetechnik realisiert, die eine präzise quantitative Messung der Konzentration der beteiligten Spezies in einer Fläche in einem Mikromischer ermöglichte. Die Fläche wurde dabei von einem Laser Lichtschnitt definiert. Durch den Einsatz gepulster Laserstrahlung war es möglich Momentaufnahmen der Verteilungen zu erfassen. Durch Aufnahme einer Vielzahl von Momentaufnahmen wurden außerdem zeitliche und örtliche Mittelungen und deren Schwankungen detektiert. Die flächige Verteilung der Standardabweichung in Kombination mit der gemessenen quantitativen Speziesverteilung lieferte neue Einsichten in die Dynamik des Mischprozesses im untersuchten Modellsystem. Der mittlere Fehler der gemessenen Konzentrationsverteilungen lag bei 4-5\%. Das entwickelte Verfahren wurde abschließend zur vollständigen Charakterisierung des Mischprozesses in der dreidimensionalen Mischkammer des Prototypen eines Multilaminationsmischers eingesetzt. Die Ergebnisse lieferten wichtige Erkenntnisse über die Gemischbildungsdynamik und die resultierende Mischungsgüte.

Eine simultane Messung des Geschwindigkeitsfeldes und der Konzentrationsverteilung erlaubt erheblich genauere Analysen des Ablaufs von Mischprozessen, als es anhand beider einzelner Messgrößen möglich wäre. Die vorgestellten Messtechniken könnten daher in Zukunft zu einem simultanen Messverfahren kombiniert werden. Die Entwicklung und Optimierung mikrofluidischer Systeme beruhte bisher zu einem großen Teil auf dem Trial-and-Error-Prinzip. Es hat sich jedoch gezeigt, dass viele Erfahrungen aus der makroskopischen Welt nicht in die deutlich kleineren Dimensionen der Mikrofluidik umgesetzt werden können. Die Grundlagenforschung, unter anderem auf Basis von berührungslosen optischen Messtechniken, wird in Zukunft eine bedeutende Rolle bei der Weiterentwicklung mikrofluidischer Systeme spielen. Dazu können die vorgestellten Messverfahren einen wesentlichen Beitrag leisten. 


\section{A. Anhang}

\section{A.1. Konstruktionszeichnungen}

Auf den folgenden Seiten finden sich die Konstruktionszeichnungen des flachen Mikromischers, der im Verlauf der vorliegenden Arbeit entwickelt wurde. 


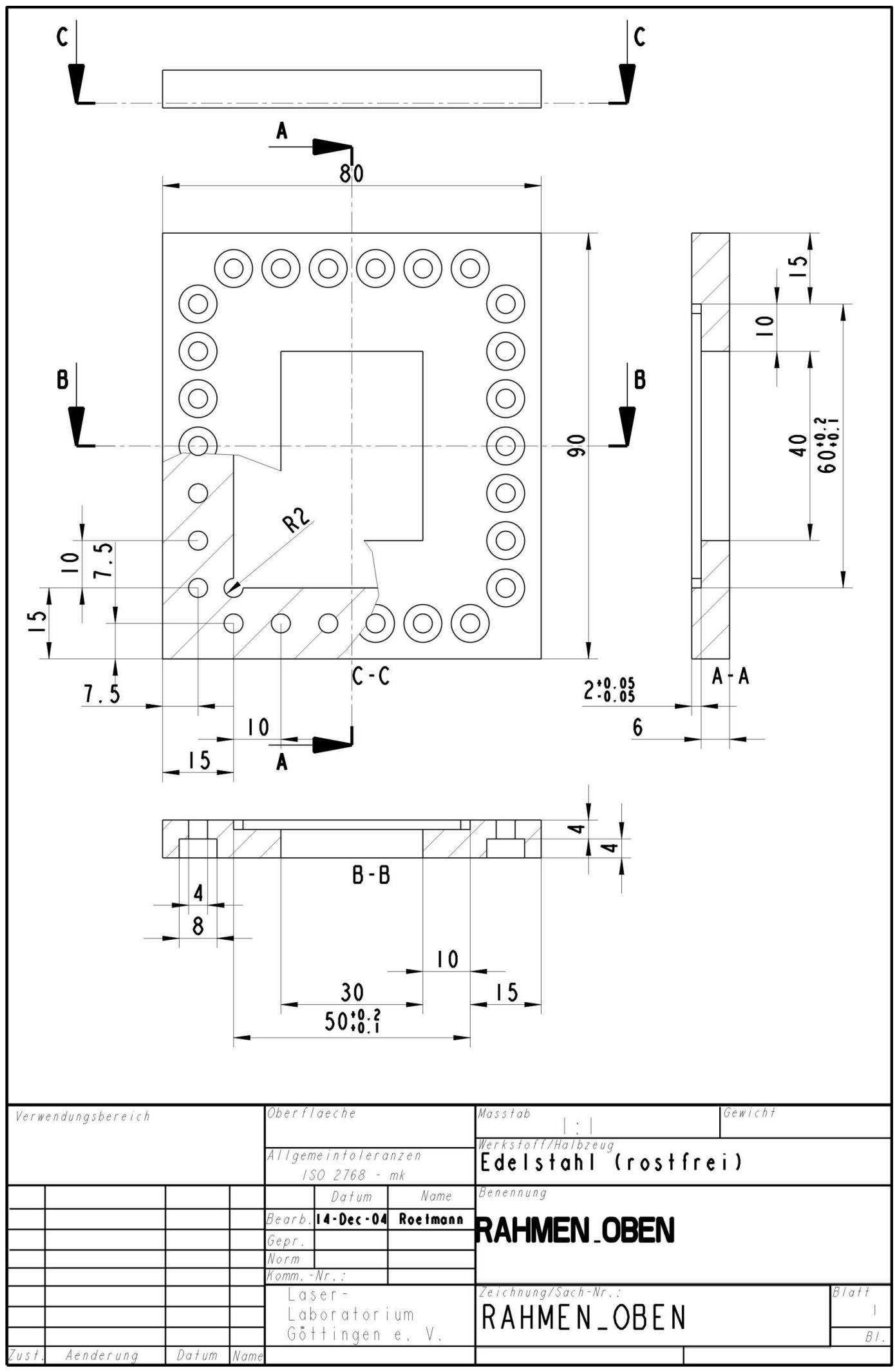

Abb. A.1.: Konstruktionszeichnung des Rahmens, Bauteil oben 


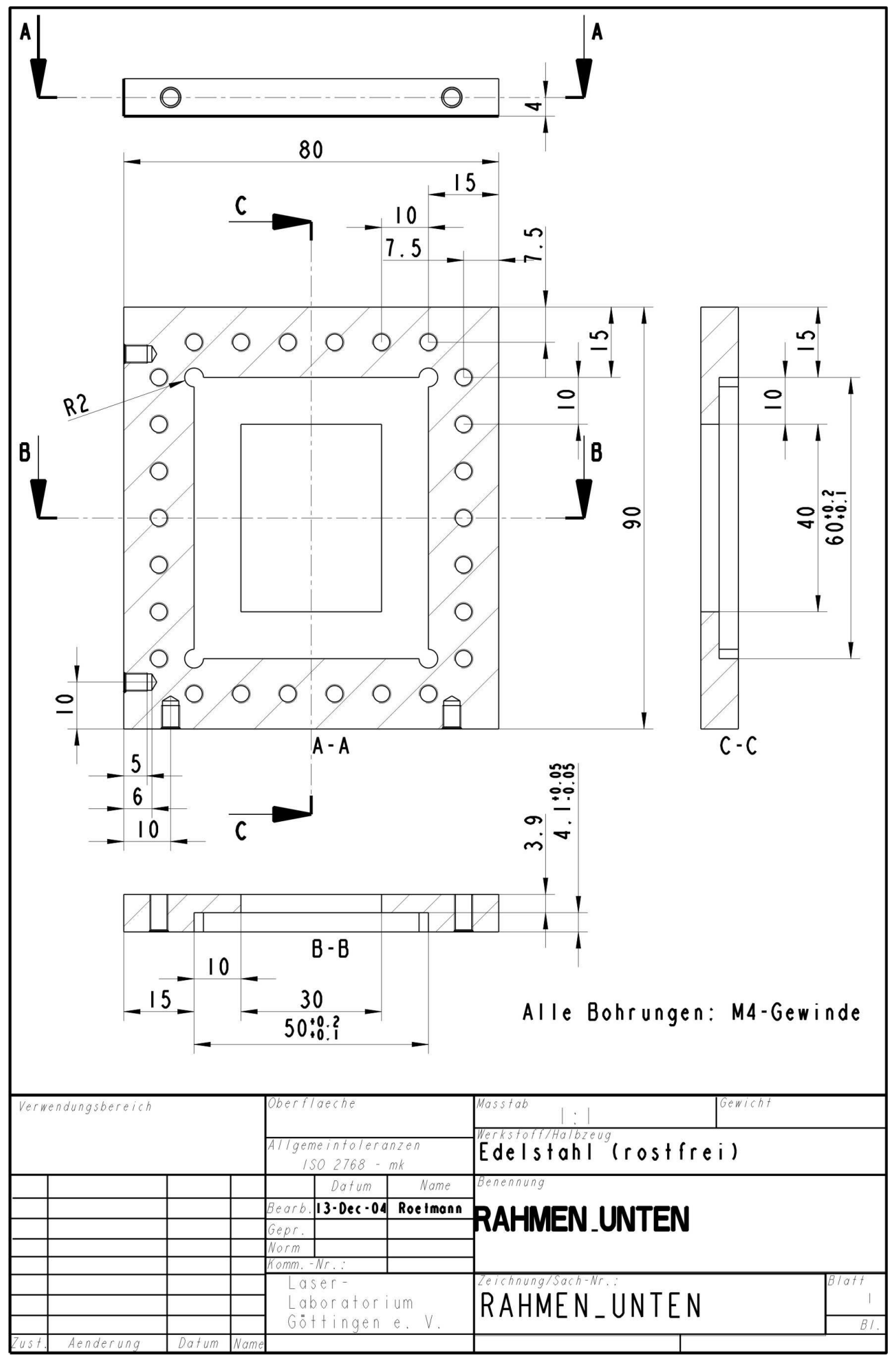

Abb. A.2.: Konstruktionszeichnung des Rahmens, Bauteil unten 


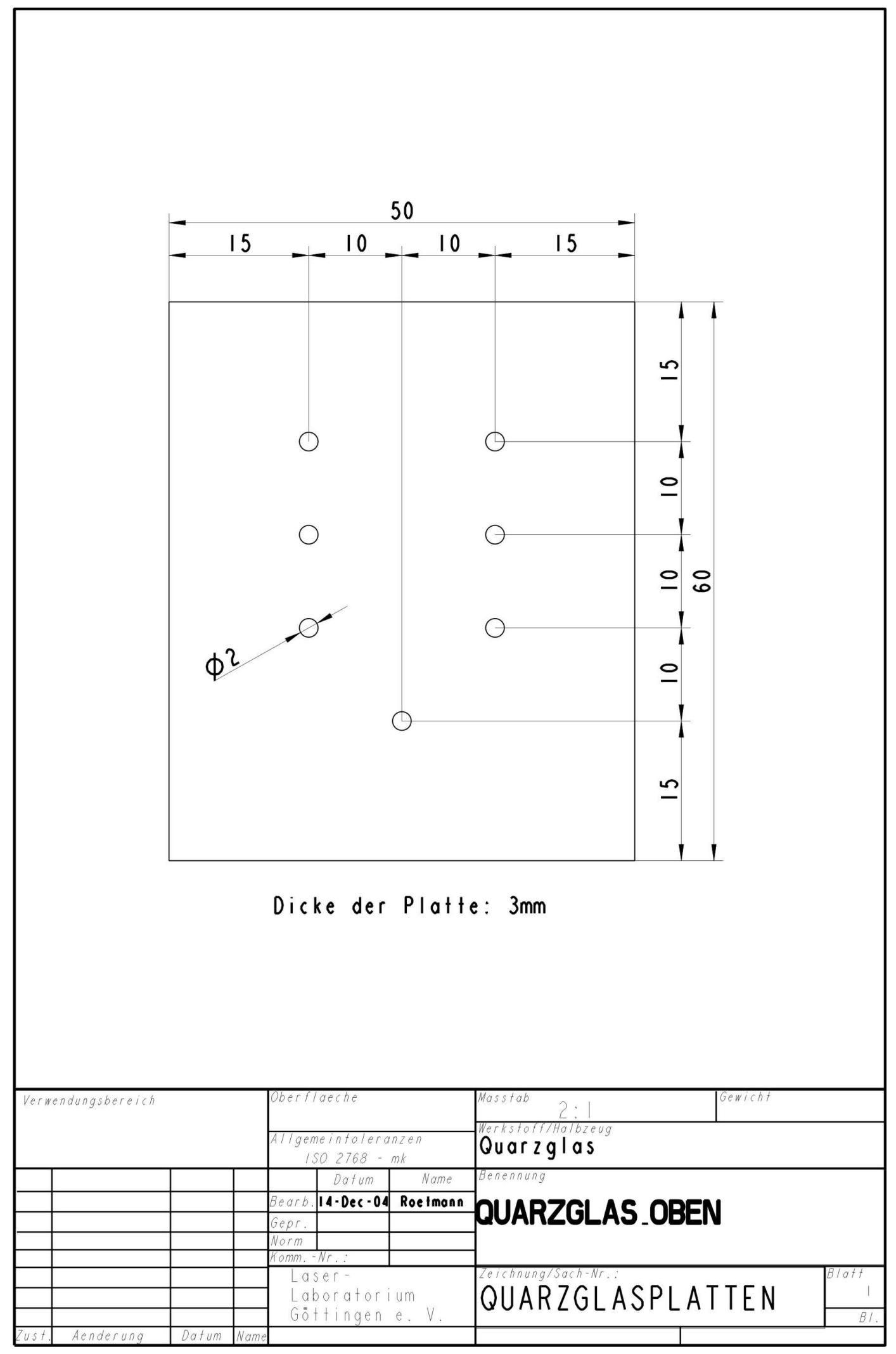

Abb. A.3.: Konstruktionszeichnung der Quarzglasplatten 


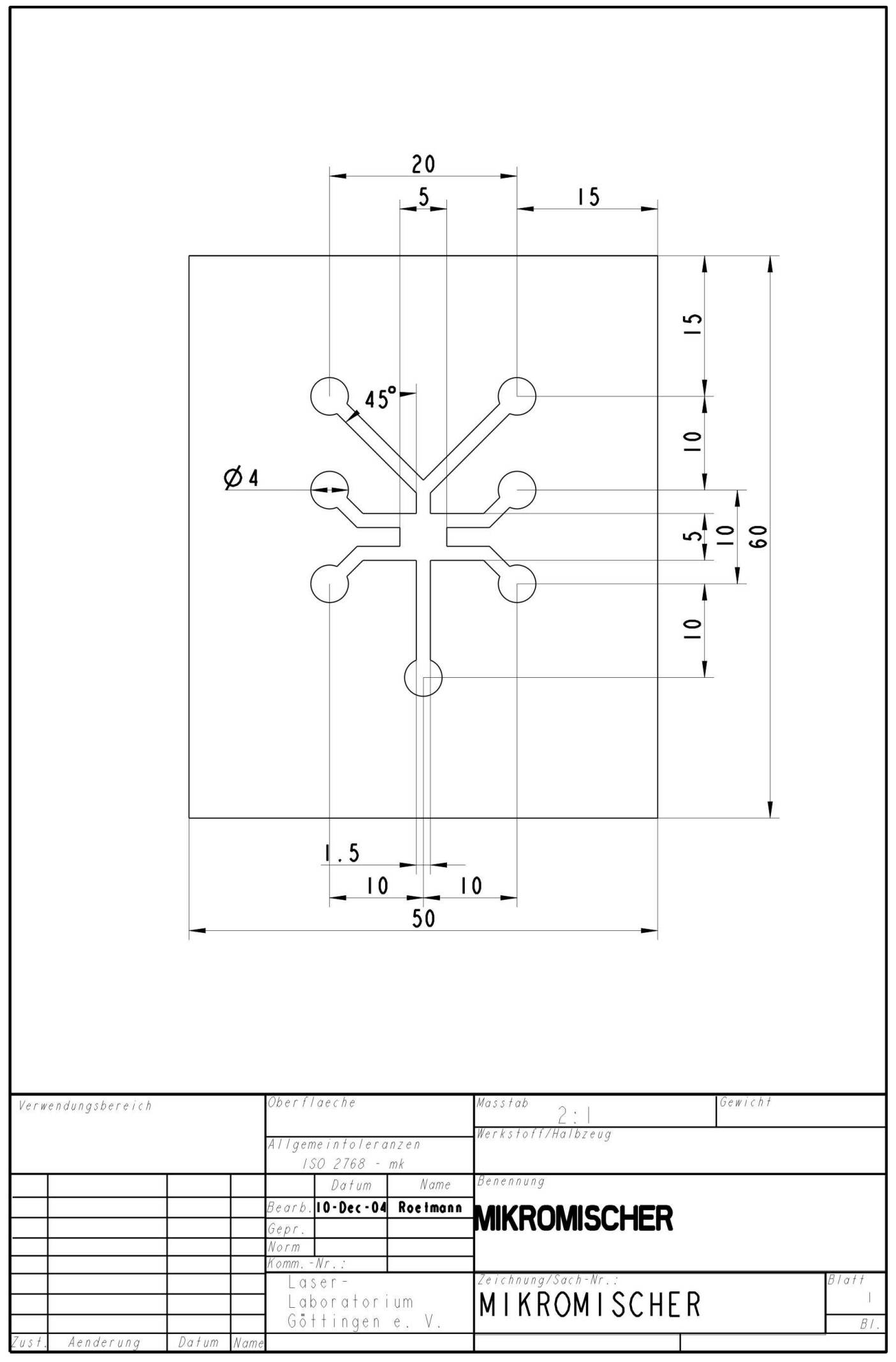

Abb. A.4.: Konstruktionszeichnung des großen Mikromischers 


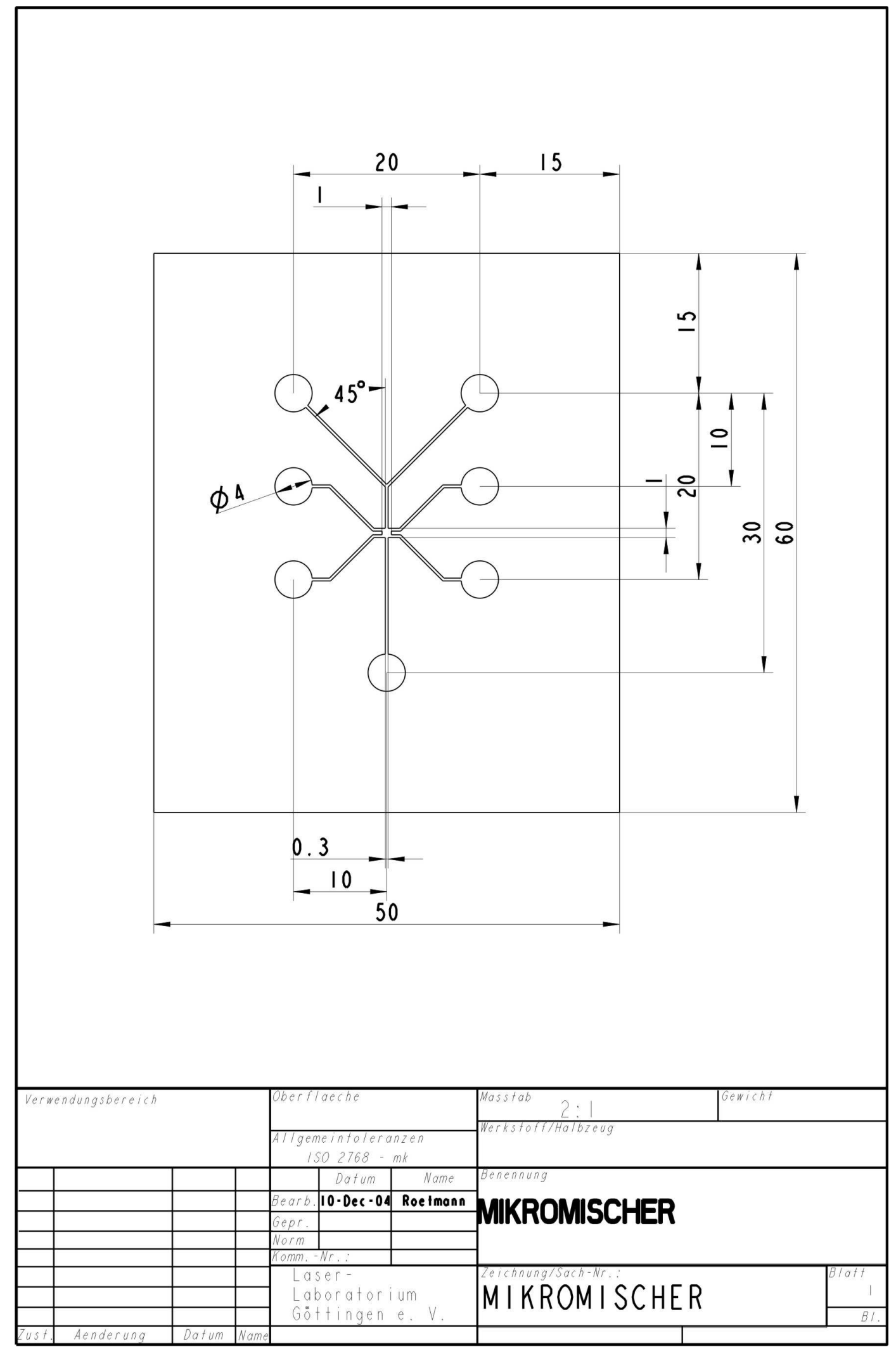

Abb. A.5.: Konstruktionszeichnung des kleinen Mikromischers 


\section{Literaturverzeichnis}

[1] Barron, J. L. ; Fleet, D.J. ; Beauchemin, S.: Performance of Optical Flow Techniques,. In: International Journal of Computer Vision 12:1 (1994), S. 43-77

[2] Bayraktar, Tuba ; Pidugu, Srikanth B.: Characterization of liquid flows in microfluidic systems. In: International Journal of Heat and Mass Transfer 49 (2006), S. 815-824. http://dx.doi.org/10.1016/j.ijheatmasstransfer.2005.11.007. - DOI 10.1016/j.ijheatmasstransfer.2005.11.007

[3] Bazile, R. ; Stepowski, D.: Measurement of the vaporization dynamics in the development zone of a burning spray by planar laser induced fluorescence and Raman scattering. In: Experiments in Fluids 16 (1994), S. 171-180

[4] Beebe, David J. ; Mensing, Glennys A. ; Walker, Glenn M.: Physics and Applications of Microfluidics in Biology. In: Annu. Rev. Biomed. Eng. 4 (2002), S. 261286. http://dx.doi.org/10.1146/annurev.bioeng.4.112601.125916. - DOI 10.1146/annurev.bioeng.4.112601.125916

[5] Bilitewski, Ursula ; Genrich, Meike ; Kadow, Sabine ; Mersal, Gaber: Biochemical analysis with microfluidic systems. In: Anal Bioanal Chem 337 (2003), S. 556-569. http://dx.doi.org/DOI10.1007/s00216-003-2179-4. - DOI DOI $10.1007 / \mathrm{s} 00216-003-2179-4$

[6] Bottausci, Frederic ; Cardonne, Caroline ; Meinhart, Carl ; Mezic, Igor: An ultrashort mixing length micromixer: The shear superposition micromixer. In: Lab on a Chip 7 (2006), S. 396-398. http://dx.doi.org/10.1039/b616104a. - DOI $10.1039 / \mathrm{b} 616104 \mathrm{a}$

[7] Bowden, Michaela ; Song, Linan ; Walt, David R.: Development of a Microfluidic Platform with an Optical Imaging Microarray Capable of Attomolar Target DNA Detection. In: Anal. Chem. 77 (2005), S. 5583-5588

[8] Burns, J. R. ; Ramshaw, C.: The intensification of rapid reactions in multiphase systems using slug flow in capillaries. In: Lab on a Chip 1 (2001), S. 10-15. http: //dx.doi.org/10.1039/b102818a. - DOI 10.1039/b102818a

[9] Chen, Chuan-Hua ; Santiago, Juan G.: A Planar Electroosmotic Micropump. In: Journal of Microelectromechanical Systems 11 (2002), S. 672-683. http://dx.doi . org/10.1109/JMEMS . 2002.805055. - DOI 10.1109/JMEMS.2002.805055 
[10] Chung, Jaewon ; Grigoropoulos, Costas P. ; Greif, Ralph: Infrared thermal velocimetry for nonintrusive flow measurement in silicon microfluidic devices. In: Review of Scientific Instruments 74 (2003), S. 2911-2917. http://dx.doi.org/ DOI : 10.1063/1.1569397. - DOI DOI: 10.1063/1.1569397

[11] Curran, Kieran ; Davies, Mark: Spectral intensity mapping and analysis of dyed microflows. In: Microfluidics and Nanofluidics 1 (2004), S. 146-154. http://dx. doi.org/10.1007/s10404-004-0022-7. - DOI 10.1007/s10404-004-0022-7

[12] Debesset, S. ; Hayden, C. J. ; Dalton, C. ; Eijkel, J. C. T. ; Manz, A.: An AC electroosmotic micropump for circular chromatographic applications. In: Lab Chip 4 (2004), S. 396-400. http://dx.doi.org/10.1039/b314123c. - DOI $10.1039 / \mathrm{b} 314123 \mathrm{c}$

[13] DürR, Manfried ; Kentsch, Jörg ; Müller, Torsten ; Schnelle, Thomas ; STELzLE, Martin: Microdevices for manipulation and accumulation of micro- and nanoparticles by dielectrophoresis. In: Electrophoresis 24 (2003), S. 722-731

[14] Ehrhard, P. ; Meisel, I.: Strömungs- und Transportprozesse in Mikrokanälen. In: Nachrichten - Forschungszentrum Karlsruhe 2-3 (2002), S. 137-142

[15] ErICKSON, David ; LI, Dongqing: Integrated microfluidic devices. In: Analytica Chimica Acta 507 (2004), S. 11-26. http://dx.doi.org/10.1016/j.aca.2003. 09.019. - DOI 10.1016/j.aca.2003.09.019

[16] Garbe, Christoph S.: Measuring and Modeling Fluid Dynamic Processesusing Digital Image Sequence Analysis. Habil. Ruprecht-Karls-Universität Heidelberg, 2006

[17] Garbe, Christoph S. ; Roetmann, Karsten ; Beushausen, Volker ; Jähne, Bernd: An optical flow MTV based technique for measuring microfluidic flow in the presence of diffusion and taylor dispersion. In: Experiments in Fluids (2007). http://dx. doi.org/10.1007/s00348-007-0435-7. - DOI 10.1007/s00348-007-0435-7

[18] Gee, K. R. ; Weinberg, E. S. ; Kozlowski, D. J.: Caged Q-rhodamine dextran: a new photoactivated fluorescent tracer. In: Bioorg Med Chem Lett 11 (2001), Aug, Nr. 16, S. 2181-2183

[19] Gendrich, C. P. ; Koochesfahani, M. M.: A spatial correlation technique for estimating velocity fields using molecular tagging velocimetry (MTV). In: Experiments in Fluids 22 (1996), S. 67-77

[20] Gendrich, C. P. ; Koochesfahani, M. M. ; Nocera, D. G.: Molecular tagging velocimetry and other novel applications of a new phosphorescent supramolecule. In: Experiments in Fluids 23 (1997), S. 361-372

[21] Gharib, M. ; Kremers, D. ; Koochesfahani, M.M. ; Kemp, M.: Leonardo's vision of flow visualization. In: Experiments in Fluids 33 (2002), S. 219-223. http: //dx.doi.org/10.1007/s00348-002-0478-8. - DOI 10.1007/s00348-002-0478-8 
[22] Haussecker, H. ; Fleet, D.J.: Computing Optical Flow with Physical Models of Brightness Variation. In: IEEE Transactions on Pattern Analysis and Machine Intelligence 23:6 (2001), S. 661-673

[23] Hertzog, David E. ; Michalet, Xavier ; Jager, Marcus ; Kong, Xiangxu ; Santiago, Juan G. ; Weiss, Shimon ; Bakajin, Olgica: Femtomole Mixer for Microsecond Kinetic Studies of Protein Folding. In: Anal. Chem. 76 (2004), S. 7169-7178. http://dx.doi.org/10.1021/ac048661s. - DOI 10.1021/ac048661s

[24] Hu, H. ; Koochesfahani, M.M.: A novel method for instantaneous, quantitative measurement of molecular mixing in gaseous flows. In: Experiments in Fluids 33 (2002), S. 202-209. http://dx.doi.org/10.1007/s00348-002-0452-5. - DOI $10.1007 / \mathrm{s} 00348-002-0452-5$

[25] Inaba, S. ; Sato, Y. ; Hishida, K. ; Maeda, M.: Flow Measurements in Microspace Using Sub-Micron Fluorescent Particles -An Effect of Brownian Motion on Velocity Detection-. In: 4th International Symposium on Particle Image Velocimetry, 2001

[26] Irisawa, G. ; Ishizuka, M. ; Sato, Y. ; Hishida, K. ; MaedA, M.: Visualization of Convective Mixing in Microchannel by Fluorescence Imaging. In: 4th International Symposium on Particle Image Velocimetry, 2001

[27] Jackman, Rebecca J. ; Floyd, Tamara M. ; Ghodssi, Reza ; Schmidt, Martin A. ; Jensen, Klavs F.: Microfluidic systems with on-line UV detection fabricated in photodefinable epoxy. In: Journal of Micromechanics and Microengineering 11 (2001), S. 263-269

[28] J̈̈Hne, Bernd: Digitale Bildverarbeitung, 6. ed. Springer, 2005

[29] Kockmann, Norbert ; Engler, Michael ; Woias, Peter: Theoretische und experimentelle Untersuchungen der Mischvorgänge in T-förmigen Mikroreaktoren. In: Chemie Ingenieur Technik 76 (2004), S. 1777-1783. http://dx.doi.org/10.1002/ cite.200407016. - DOI 10.1002/cite.200407016

[30] Koochesfahani, M. M. ; Cohn, R. K. ; Gendrich, C. P. ; Nocera, D. G.: Molecular Tagging Diagnostics for the Study of Kinematics and Mixing in LiquidPhase Flows. In: 8th International Symposium on Applications of Laser Techniques to Fluid Mechanics, 1996, S. 1.2.1-1.2.12

[31] Koochesfahani, M. M. ; Goh, A. C. ; Schock, H. J.: Molecular Tagging Velocimetry (MTV) and Its Automotive Applications. In: Lecture Notes in Applied and Computational Mechanics 19 (2004), S. 143-155

[32] Koochesfahani, Manoochehr ; Cohn, Richard ; MacKinnon, Colin: Simultaneous whole-field measurements of velocity an concentration fields using a combination of MTV and LIF. In: Measurement science and technology 11 (2000), S. $1289-1300$ 
[33] Koochesfahani, Manoochehr M. ; Nocera, Daniel G.: Molecular tagging velocimetry maps fluid flows. In: Laser Focus World - (2001), S. 103-108

[34] Krüger, S. ; Grünefeld, G. ; Arndt, S. ; Hentschel, W.: Planar velocity measurements of the gas and liquid phase in dense sprays by flow tagging. In: 10th International Symposium on Applications of Laser Techniques to Fluid Mechanics, 2000

[35] Lee, Moonkwon ; Lee, Jong-Phil ; Rhee, Hakjune ; Choo, Jaebum ; ChaI, Young G. ; LEE, Eun K.: Applicability of laser-induced Raman microscopy for in situ monitoring of imine formation in a glass microfluidic chip. In: Journal of Raman Spectroscopy 34 (2003), S. 737-742. http://dx.doi.org/10.1002/jrs.1038. DOI $10.1002 /$ jrs.1038

[36] Lempert, W. R. ; Magee, K. ; Ronney, P. ; Gee, K. R. ; Haugland, R. P.: Flow tagging velocimetry in incompressible flow using photo-activated nonintrusive tracking of molecular motion (PHANTOMM). In: Experiments in Fluids 18 (1995), S. 249-257

[37] Lempert, Walter R. ; HARRIS, Scott R.: Flow tagging velocimetry using caged dye photo-activated fluorophores. In: Measurement Science and Technology 11 (2000), S. $1251-1258$

[38] Leung, Shee-Ann ; Winkle, Richard F. ; Wootton, Robert C. R. ; DeMello, Andrew J.: A method for rapid reaction optimisation in continuous-flow microfluidic reactors using online Raman spectroscopic detection. In: The Analyst 130 (2004), S. 46-51. http://dx.doi.org/10.1039/b412069h. - DOI 10.1039/b412069h

[39] Lin, Che-Hsin ; Tsai, Chien-Hsiung ; Fu, Lung-Ming: A rapid three-dimensional vortex micromixer utilizing self-rotation effects under low Reynolds number conditions. In: Journal of Micromechanics and Microengineering 12 (2005), S. 935943. http://dx.doi.org/10.1088/0960-1317/15/5/006. - DOI 10.1088/0960$1317 / 15 / 5 / 006$

[40] Liu, Robin H. ; Stremler, Mark A. ; Sharp, Kendra V. ; Olsen, Michael G. ; Santiago, Juan G. ; Adrian, Ronald J. ; Aref, Hassan ; Beebe, David J.: Passive Mixing in a Three-Dimensional Serpentine Microchannel. In: Journal of Microelectromechanicalsystems 9 (2000), S. 190-197

[41] Locascio, Laurie E.: Microfluidic mixing. In: Anal Bioanal Chem 379 (2004), S. 325-327. http://dx.doi.org/10.1007/s00216-004-2630-1. - DOI $10.1007 / \mathrm{s} 00216-004-2630-1$

[42] Lum, C.L. ; Koochesfahani, M.M. ; McGrath, J.J.: Measurements Of The Velocity Field With MTV During The Solidification Of An Alloy Analog With Mushy Region. In: Proceedings of ASME IMECE, 2001 
[43] Magennis, Steven W. ; Graham, Emmelyn M. ; Jones, Anita C.: Quantitative Spatial Mapping of Mixing in Microfluidic Systems. In: Angew. Chem. Int. Ed. 44 (2005), S. 6512-6516. http://dx.doi.org/10.1002/anie.200500558. - DOI 10.1002/anie. 200500558

[44] Malarski, Anna ; Egermann, Jan ; Zehnder, Julia ; Leipertz, Alfred: Simultaneous application of single-shot Ramanography and particle image velocimetry. In: Optics letters 31 (2006), S. 1005-1007

[45] Manz, Andreas ; EiJkel, Jan C. T.: Miniaturization and chip technology.What can we expect? In: Pure Appl. Chem. 73 (2001), S. 1555-1561

[46] Maynes, D. ; WebB, A. R.: Velocity profile characterization in sub-millimeter diameter tubes using molecular tagging velocimetry. In: Experiments in Fluids 32 (2002), S. 3-15. http://dx.doi.org/10.1007/s003480100290. - DOI $10.1007 / \mathrm{s} 003480100290$

[47] McDonald, J. C. ; Duffy, David C. ; Anderson, Janelle R. ; Chiu, Daniel T. ; Wu, Hongkai ; Schueller, Olivier J. A. ; Whitesides, George M.: Fabrication of microfluidic systems in poly(dimethylsiloxane). In: Electrophoresis 21 (2000), S. $27-40$

[48] Meinhart, C. D. ; Wereley, S. T. ; Gray, M. H. B.: Volume illumination for twodimensional particle image velocimetry. In: Measurement Science and Technology 11 (2000), S. 809-814

[49] Mogensen, Klaus B. ; Klank, Henning ; Kutter, Jörg P.: Recentdevelopments in detection for microfluidic systems. In: Electrophoresis 25 (2004), S. 3498-3512. http://dx.doi.org/10.1002/elps.200406108. - DOI 10.1002/elps.200406108

[50] Mosier, B. P. ; Molho, J. I. ; Santiago, J. G.: Photobleached-fluorescence imaging of microflows. In: Experiments in Fluids 33 (2002), S. 545-554. http: //dx.doi.org/10.1007/s00348-002-0486-8. - DOI 10.1007/s00348-002-0486-8

[51] NGuyen, Nam-Trung; Wu, Zhigang: Micromixers-a review. In: Journal of Micromechanics and Microengineering 15 (2005), S. R1-R16. http://dx.doi.org/10. 1088/0960-1317/15/2/R01. - DOI 10.1088/0960-1317/15/2/R01

[52] Oddy, M. H. ; Santiago, J. G. ; Mikkelsen, J. C.: Electrokinetic Instability Micromixing. In: Anal. Chem 73 (2001), S. 5822-5832. http://dx.doi.org/10. 1021/ac0155411. - DOI 10.1021/ac0155411

[53] Paege, Brian M. ; Emrich, Charles A. ; Wedemayer, Gary J. ; Scherer, James R. ; MATHIES, Richard A.: High throughput DNA sequencing with a microfabricated 96-lane capillary array electrophoresis bioprocessor. In: PNAS 99 (2001), S. 574-579. http://dx.doi.org/10.1073/pnas.012608699. - DOI 10.1073/pnas.012608699 
[54] Park, Taehan ; Lee, Moonkwon ; Choo, Jaebum ; Kim, Yang S. ; Lee, Eun K. ; Kim, Duck J. ; LeE, Sang-Hoon: Analysis of Passive Mixing Behavior in a Poly(dimethylsiloxane) Microfluidic Channel Using Confocal Fluorescence and Raman Microscopy. In: Applied Spectroscopy 58 (2004), S. 1172-1179

[55] Park, Taehan ; Lee, Sangyeop ; Seong, Gi H. ; Choo, Jaebum ; Lee, Eun K. ; Kim, Yang S. ; Ji, Won H. ; Hwang, Seung Y. ; Gweon, Dae-Gab ; LeE, Sanghoon: Highly sensitive signal detection of duplex dye-labelled DNA oligonucleotides in a PDMS microfluidic chip: confocal surface-enhanced Raman spectroscopic study. In: Lab Chip 5 (2005), S. 437-442. http://dx.doi.org/10.1039/b414457k. - DOI $10.1039 / \mathrm{b} 414457 \mathrm{k}$

[56] Paul, P. H. ; Garguilo, M. G. ; Rakestraw, D. J.: Imaging of Pressure- and Electrokinetically Driven Flows through Open Capillaries. In: Anal. Chem. 70 (1998), S. 24592467

[57] Pfadler, Sebastian ; Beyrau, Frank ; Löffler, Micha ; Leipertz, Alfred: Application of a beam homogenizer to planar laser diagnostics. In: Optics Express 14 (2006), S. 10171-10180

[58] Rossier, Joël S. ; Vollet, Christine ; Carnal, Amanda ; Lagger, Grégoire ; GoBry, Véronique ; Girault, Hubert H. ; Michela, Philippe ; Reymonda, Frédéric: Plasma etched polymer microelectrochemical systems. In: Lab on a chip 2 (2002), S. 145-150. http://dx.doi.org/10.1039/b204063h. - DOI 10.1039/b204063h

[59] SADR, Reza ; KLEWiCKI, Joseph C.: An experimental investigation of the near-field flow development in coaxial jets. In: Physics of Fluids 15 (2003), S. 1233-1245

[60] Salmon, Jean-Baptiste ; Ajdari, Armand ; Tabelin, Patrick ; Servant, Laurent ; TAlaga, David ; JoAnicot, Mathieu: In situ Raman imaging of interdiffusion in a microchannel. In: Applied Physics Letters 86 (2005), S. 1-3

[61] Santiago, J. G.: Electroosmotic Flows in Microchannels with Finite Inertial and Pressure Forces. In: Anal. Chem. 73 (2001), S. 2353-2365. http://dx.doi.org/ 10.1021/ac0101398. - DOI 10.1021/ac0101398

[62] Scharr, H.: Optimal Filters for Extended Optical Flow. In: Lecture Notes in Computer Science. Springer Verlag, 2007 (Lecture Notes in Computer Science)

[63] Shinohara, Etsuo ; Tajima, Nobuyoshi ; Suzuki, Hirobumi ; Funazaki, Jun: Microfabricated Free Flow Electrophoresis Module for Sample Preparations. In: Analytical Sciences 17 (2001), S. 441-443

[64] ShoJI, Shuchi ; EsAshi, Masayoshi: Microflow devices and systems. In: J. Micromech. Microeng. 4 (1994), S. 157-171 
[65] Sinton, David: Microscale flow visualization. In: Microfluid Nanofluid 1 (2004), S. 2-21. http://dx.doi.org/10.1007/s10404-004-0009-4. - DOI 10.1007/s10404004-0009-4

[66] Sinton, David ; Erickson, David ; Li, Dongqing: Photo-injection based sample design and electroosmotic transport in microchannels. In: Journal of Micromechanics and Microengineering 12 (2002), S. 898-904

[67] Stier, B. ; Koochesfahani, M. M.: Molecular Tagging Velocimetry (MTV) measurements in gas phase flows. In: Experiments in Fluids 26 (1999), S. 297-304

[68] Suzuki, Hiroaki ; Ho, Chih-Ming: A Magnetic Force Driven Chaotic Micro-Mixer. In: The Fifteenth IEEE International Conference on Micro Electro Mechanical Systems, 2002, 2002, S. 40-43

[69] TAYlor, Geoffrey: Dispersion of Soluble Matter in Solvent Flowing Slowly through a Tube. In: Proceedings of the Royal Society of London. Series A, Mathematical and Physical Sciences 219 (1953), S. 186-203

[70] TAYlor, Geoffrey: Conditions under Which Dispersion of a Solute in a Stream of Solvent can be Used to Measure Molecular Diffusion. In: Proceedings of the Royal Society of London. Series A, Mathematical and Physical Sciences 225 (1954), S. $473-477$

[71] Viskari, Pertti J. ; Landers, James P.: Unconventional detection methods for microfluidic devices. In: Electrophoresis 27 (2006), S. 1797-1810. http://dx.doi. org/10.1002/elps.200500565. - DOI 10.1002/elps.200500565

[72] Vo-Dinh, T. ; Stokes, D. L. ; Griffin, G. D. ; Volkan, M. ; Kim, U. J. ; M.I.Simon: Surface-enhanced Raman Scattering (SERS) Method and Instrumentation for Genomics and Biomedical Analysis. In: Journal of Raman Spectroscopy 30 (1999), S. 785-793

[73] Wabuyele, Musundi B. ; Yan, Fei ; Grif?n, Guy D. ; Vo-Dinh, Tuan: Hyperspectral surface-enhanced Raman imaging of labeled silver nanoparticles in single cells. In: Review of Scientific Instruments 76 (2005), S. 1-7. http://dx.doi.org/ 10.1063/1.1938667. - DOI 10.1063/1.1938667

[74] Wood, Bayden R. ; Langford, Steven J. ; Cooke, Brian M. ; Glenister, Fiona K. ; Lim, Janelle ; McNaughton, Don: Raman imaging of hemozoin within the food vacuole of Plasmodium falciparum trophozoites. In: FEBS Letters 554 (2003), S. 247-252. http://dx.doi .org/10.1016/S0014-5793(03)00975-X. DOI 10.1016/S0014-5793(03)00975-X

[75] Yamamoto, T. ; Inaba, S. ; Sato, Y. ; Hishida, K. ; Maeda, M.: Measurements in microchannel by laser induced molecular tagging and micro-PIV. In: 11th International Symposium on Applications of Laser Techniques to Fluid Mechanics, 2002 
[76] Yea, Kwon hae ; Lee, Sangyeop ; Kyong, Jin B. ; Choo, Jaebum ; LeE, Eun K. ; Joo, Sang-Woo ; LEE, Sanghoon: Ultra-sensitive trace analysis of cyanide water pollutant in a PDMS microfluidic channel using surface-enhanced Raman spectroscopy. In: The Analyst 130 (2005), S. 1009-1011. http://dx.doi.org/10.1039/b501980j. - DOI 10.1039/b501980j

[77] Zeng, Shulin ; Chuan-Hua Chen, James C. Mikkelsen J. ; Santiago, Juan G.: Fabrication and characterization of electroosmotic micropumps. In: Sensors and Actuators 79 (2001), S. 107-114

[78] Zhao, Haiying ; Yuan, Bo ; Dou, Xiaoming: The effects of electrostatic interaction between biological molecules and nano-metal colloid on near-infrared surfaceenhanced Raman scattering. In: Journal Of Optics A: Pure And Applied Optics 6 (2004), S. 900-905. http://dx.doi.org/10.1088/1464-4258/6/9/013. - DOI $10.1088 / 1464-4258 / 6 / 9 / 013$

[79] Zhou, Xiaomian ; Liu, Dayu ; Zhong, Runtao ; Dai, Zhongpeng ; Wu, Dapeng ; WAng, Hui ; Du, Yuguang ; XIA, Zhinan ; Zhang, Liping ; MeI, Xiaodai ; Lin, Bingcheng: Determination of SARS-coronavirus by a microfluidic chip system. In: Electrophoresis 25 (2004), Sep, Nr. 17, 3032-3039. http://dx.doi.org/10.1002/ elps.200305966. - DOI 10.1002/elps.200305966 


\section{Lebenslauf}

\section{Persönliche Daten}

$\begin{array}{ll}\text { Name } & \text { Dipl. Phys. Karsten Roetmann } \\ \text { Adresse } & \text { Gaußstraße 13 } \\ & 37083 \text { Göttingen } \\ \text { Geburtsort } & \text { Nordhorn } \\ \text { Geburtsdatum } & 01.03 .1978 \\ \text { Staatsangehörigkeit: } & \text { deutsch }\end{array}$

\section{Schulische Ausbildung}
1984-1988
Grundschule Altendorf, Nordhorn
1988-1990
Orientierungsstufe Deegfeld, Nordhorn
1990-1997
Gymnasium am Stadtring, Nordhorn
1997
Schulabschluss mit Abitur

\section{Grundwehrdienst / Zivildienst}

1997-1998

Zivildienst im Marienkrankenhaus, Nordhorn

\section{Studium}

1998-2003

Georg-Augustus-Universität, Göttingen

Fachrichtung Physik, Diplom

2002-2003

Diplomarbeit am Laser-Laboratorium Göttingen e.V.

2003

Abschluss Diplom Physik

2003-heute

Promotion am Laser-Laboratorium Göttingen e.V. 